Portland State University

PDXScholar

Fall 12-2-2013

\title{
Batterer Intervention Programs' Response to State Standards
}

Ashley Lynn Boal

Portland State University

Follow this and additional works at: https://pdxscholar.library.pdx.edu/open_access_etds

Part of the Domestic and Intimate Partner Violence Commons, and the Health Services Administration Commons

Let us know how access to this document benefits you.

Recommended Citation

Boal, Ashley Lynn, "Batterer Intervention Programs' Response to State Standards" (2013). Dissertations and Theses. Paper 1504.

https://doi.org/10.15760/etd.1516

This Dissertation is brought to you for free and open access. It has been accepted for inclusion in Dissertations and Theses by an authorized administrator of PDXScholar. Please contact us if we can make this document more accessible: pdxscholar@pdx.edu. 


\title{
Batterer Intervention Programs'
}

Response to State Standards

by

Ashley Lynn Boal

A dissertation submitted in partial fulfillment of the requirements for the degree of

\author{
Doctor of Philosophy \\ in \\ Applied Psychology
}

Dissertation Committee: Eric Mankowski, Chair

Kris Henning

Kimberly Kahn

Cynthia Mohr

Portland State University

2013 


\begin{abstract}
The study of policy implementation has recently garnered research and federal attention highlighting the importance of implementation in achieving desired policy and program outcomes (Durlak \& DuPre, 2008; Meyers, Durlak \& Wandersman, 2012; National Institutes of Health, 2013). Psychology is one discipline that is well poised to guide the study of policy implementation as it can inform the creation, development, and outcomes associated with the introduction of a policy (Esses \& Dovidio, 2011; Fischhoff, 1990). Given that batterer intervention programs (BIPs) have been developed to prevent future intimate partner violence (IPV) and improve victim safety, ensuring these programs have successfully implemented state standards for practice is immensely important. Despite the widespread use of state standards to guide BIP practices (Maiuro \& Eberle, 2008), only one study (Boal, 2010) has assessed the extent to which BIPs comply with standards and no research has evaluated program responses to standards or the process by which implementation occurs. Given this, the current study focused on four areas of inquiry: (1) program compliance with state standards; (2) current and former BIP representatives' response to standards, including the social psychological constructs of actual control, perceived control, retrospective accounts of attitude change, absoluteness, and legitimacy; (3) program compliance as it relates to these responses; and (4) the process of implementing standards. In order to address these topics, key program representatives were assessed using a sequential mixed-methods design, which consisted of a preliminary quantitative phase (i.e., Phase One $)(n=35$, response rate $=74 \%)$ and principal qualitative phase (i.e., Phase Two) (current providers: $n=13$, response rate $=$
\end{abstract}


87\%; former providers: $n=5$, response rate $=100 \%$ (Morgan, 1998). Findings from Phase One indicate that programs complied with $75 \%$ of the assessed components of standards. Phase Two findings suggest that participants primarily voiced experiences with the standards consistent with a lack of actual control, perceived control, and legitimacy. Contrary to hypotheses a statistically reliable difference in actual control, perceived control, and legitimacy were not detected across high and low compliance participants. Participants retrospectively described responses to the standards consistent with changing and maintaining negative attitudes towards the standards (31\% and $31 \%$ respectively) and as hypothesized, those who shifted negative initial attitudes to be positive (i.e., a proxy for rationalization) were primarily from high compliance programs (75\%) and those who maintained negative attitudes (i.e., a proxy for reactance) were all from low compliance programs $(100 \%)$. While participants generally perceived the standards as primarily absolute, this construct did not differentiate those who changed and maintained negative attitudes as predicted. Participants' utilized diverse strategies to implement the standards and have changed or attempted to change many program characteristics to better comply with state standards. Participants have experienced diverse enablers to compliance (e.g., positive community collaborations; participation in the research process) and barriers to compliance (e.g., negative or lack of community collaborations; challenges understanding the standards) while attempting to implement standards. Suggestions to better facilitate compliance aligned with the enablers and barriers and centered on the need for positive information-sharing relationships among providers. Finally, former providers tended to disagree that the standards were the 
primary reason for program closure. Together, these findings provide valuable insight into the manifestation of common social psychological constructs during the policy implementation process, as well as information regarding the logistics of implementation. The information gathered in this study can be applied to better understand the role of actual control, perceived control, retrospective accounts of attitude change, absoluteness, and legitimacy, as they are experienced in the real world in relation to an actual policy. This extends the study of these constructs out of a laboratory and experimental context and suggests aspects of these constructs that may be relevant in applied settings. Further, data regarding the policy implementation process is useful to inform policymakers about the diverse steps that can be taken to assist implementation efforts and increase compliance. 


\section{Acknowledgements}

This dissertation study could not have been accomplished without the significant contributions of several key individuals. First, I would like to acknowledge and thank my dissertation chair and graduate mentor, Dr. Eric Mankowski. Dr. Mankowski has been instrumental in providing guidance and encouraging growth as a scholar throughout the dissertation project and my graduate studies more generally. His support for this project and feedback to improve the study has been immeasurably useful and constructive. Second, I would like to thank Amber Cummings and Kathleen Russell for their significant role in this dissertation study. Their work transcribing and coding interview data was invaluable and this study could not have been completed without their dedication and perseverance. Third, I would like to acknowledge my dissertation committee for their thoughtful suggestions and feedback to improve and expand this project. Fourth, I would like to acknowledge the Oregon Batterer Intervention Program Standards Advisory Committee for their input related to the research questions and data collection tools, as well as their general support for the study. Fifth, this study could not have been completed without the grant support provided by the Society for Community Research and Action's Public Policy Initiative Grant. Finally, I would like to send my

most sincere thanks to the participants of the study. These participants each took time out of their incredibly busy schedules to complete surveys and/or interviews. Their honesty and candor has been immensely valuable in gaining a clear picture of provider experiences and without their input this would not have been possible. 
Table of Contents

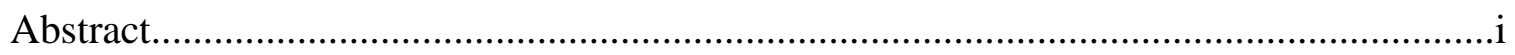

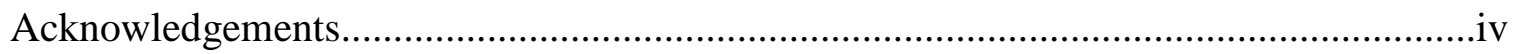

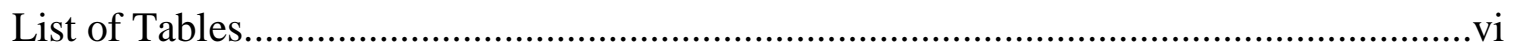

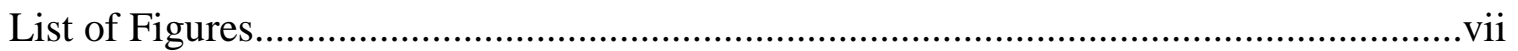

Chapter 1

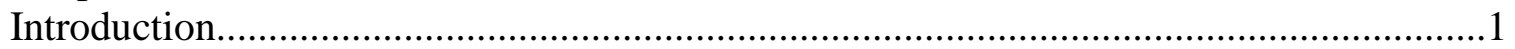

Chapter 2

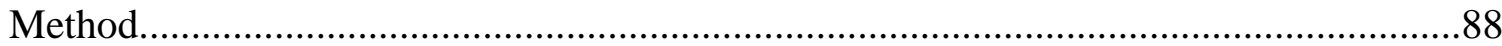

Chapter 3

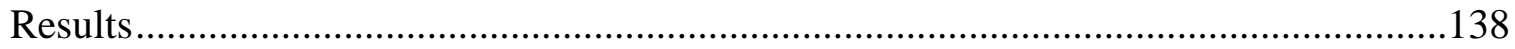

Chapter 4

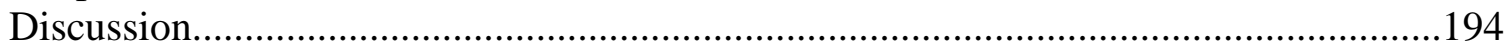

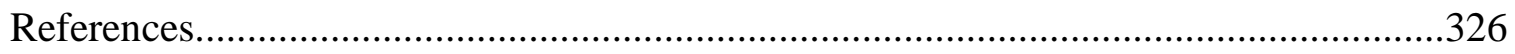

Appendices

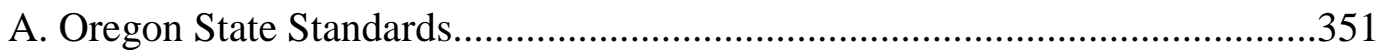

B. 2011 Oregon Batterer Intervention Program Survey....................................388

C. Batterer Intervention Program SB 81 Interview Guide................................410

D. No Longer Functioning Batterer Intervention

Program SB 81 Phone Interview Guide ...................................................417

E. Interview Codebook...........................................................................419

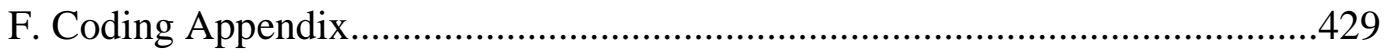




\section{List of Tables}

Table 1. Cohen's Kappa for Qualitative Coding..........................................................291

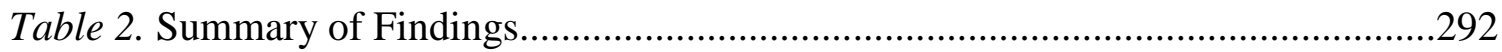

Table 3. Compliance with Standards Ranked by \% in Compliance..............................295

Table 4. Compliance with Program Logistics........................................................298

Table 5. Compliance with Training of Facilitators....................................................299

Table 6. Compliance with Program Intervention Strategies........................................300

Table 7. Compliance with Policies Relating to Victims and Partners.............................301

Table 8. Compliance with Community Collaboration Requirements.............................302

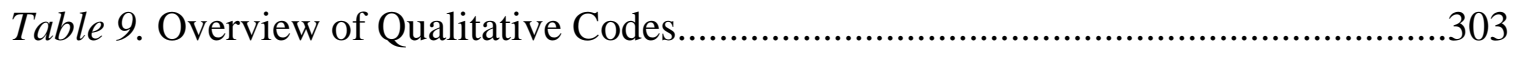

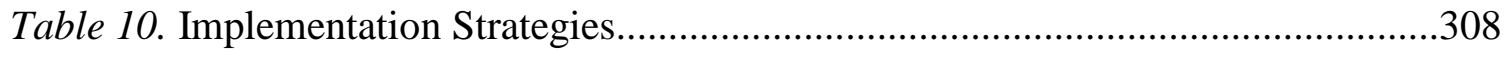

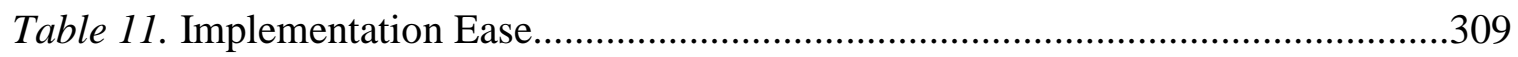

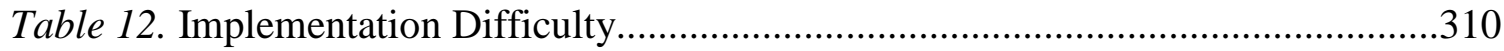

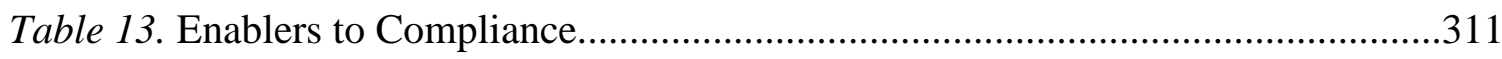

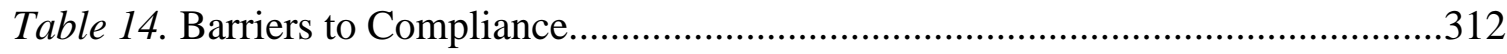

Table 15. Support Needed for Compliance............................................................ 313

Table 16. Comparison of High and Low Compliance Programs....................................314 


\section{List of Figures}

Figure 1. Locating State Standards for BIPs within the

Criminal Justice Response to IPV.

Figure 2. Conceptual Framework: Examining BIP Directors'

Response to State Standards.............................................................. 316

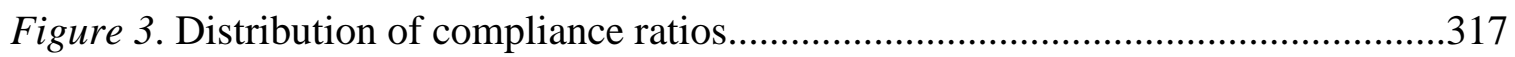

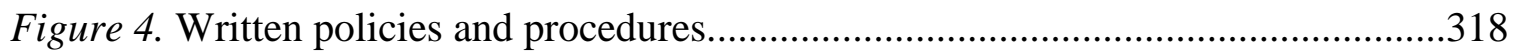

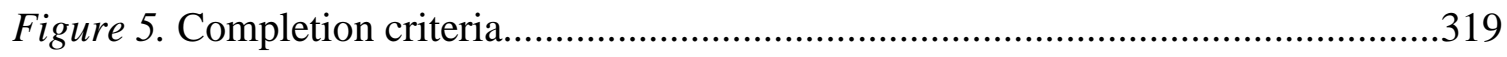

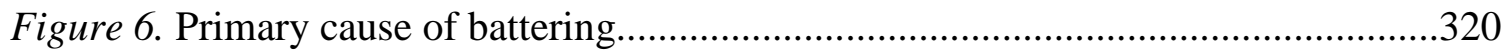

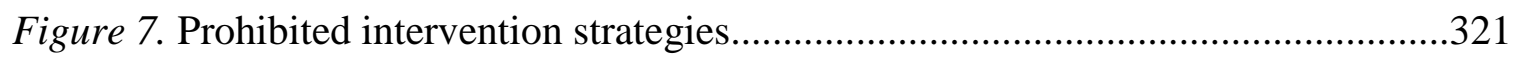

Figure 8. Approved intervention strategies....................................................... 322

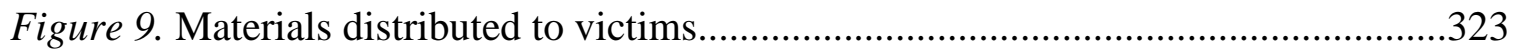

Figure 10. Collaboration with victim advocates and domestic violence council............324

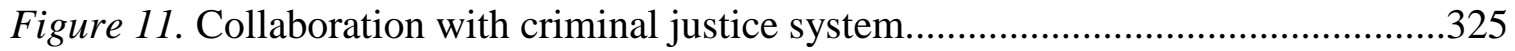


Batterer Intervention Programs’ Response to State Standards

\section{Chapter 1: Introduction}

\section{Overview}

The current study aims to build on previous research to better understand the impact of state legislation directed at batterer intervention programs (BIPs) and examine key program staff members' responses to this policy. While many studies of intimate partner violence (IPV) focus on victims of abuse, this study aspired to inform efforts to prevent IPV, increase social justice, and avoid victim-blaming by examining the perpetrators of abuse. This is accomplished by investigating interventions for offenders of IPV known as BIPs. Though studies have been conducted examining individual outcomes for participants in these programs, fewer studies have considered the context in which these individual outcomes occur. The current study not only aims to understand the context of BIPs by examining programs' current practices and policies, but also attempts to understand how the larger climate of state policy affects these programs.

This study surveyed key program staff members of all BIPs in the state of Oregon $(n=35$, response rate $=74 \%)$ to examine the extent to which the policy of state standards has been successfully implemented in the state of Oregon. Next, this study conducted extensive interviews with a subset of key program staff members $(n=13$, response rate $=$ $87 \%$ ) in order to identify and describe the process of implementation and perceptions of the standards. The interviews attempted to gather information regarding implementation, including the process by which programs became aware of standards; their experiences related to implementation of the standards; current program functioning in relation to the 
standards; barriers and/or facilitators of compliance with the regulation; perceived control over the content and scope of the standards; change or lack of change in negative attitudes towards the standards; perceptions of how absolute or flexible the standards are; perceived legitimacy of the standards; key program staff members' views of standards overall; and key program staff members' opinions about specific aspects of the standards. This study was the first to go beyond examining compliance with standards and instead investigated the entire process of and experience with policy implementation in a BIP setting. In order to fully appreciate the process and outcomes of policy implementation for BIPs, relevant social psychological constructs that might contribute to the understanding of how key program staff members have attempted implementation and the extent to which adherence to standards has been achieved were utilized as analytic lenses. Further, the current study utilized a social action research approach in order to provide a platform for key program staff members affected by the standards to describe their experiences adapting to the standards and an avenue for them to offer feedback to policymakers.

\section{Intimate Partner Violence}

Intimate partner violence (IPV) is a significant social problem that has profound physical, psychological, and economic effects for many individuals, particularly women. The Centers for Disease Control and Prevention (CDCP) defines IPV as "physical, sexual, or psychological harm by a current or former partner or spouse" (CDCP, 2010). Inherent in this definition are four primary forms of IPV: physical violence, sexual violence, threats of violence, and psychological/emotional violence (CDCP, 2010; 
Saltzman, Fanslow, McMahon \& Shelley, 2002). While the CDCP provides one widely used definition of IPV, it is important to recognize that across agencies and research studies the definitions of IPV differ. In some arenas all types of abuse are accounted for when determining the number of individuals affected (i.e., Coker, Smith, McKeown \& King, 2000; Thompson, Bonomi, Anderson, Reid, Dimer, Carrell \& Rivara, 2006), while others limit their criteria to physical assault (i.e., Tjaden \& Thoennes, 1998). Given the discrepancies in definitions for IPV, it is perhaps not surprising that estimates of the number of individuals affected by IPV vary widely (Saltzman et al., 2002).

With this caveat in mind, studies have found that one-half to two million individuals are victims of IPV in the United States each year (Catalano, 2007; Tjaden \& Thoennes, 2000) and approximately 25 to $54 \%$ of the female population experiences some type of violence committed by a significant other in their lifetime (Coker et al., 2000; Tjaden \& Thoennes, 1998; Thompson et al., 2006). Additionally, between 2001 and 2005, IPV accounted for $22 \%$ of non-fatal violent crimes against women in the United States (Catalano, 2007). Men are the victim in the majority of murders in the United States. For instance, in 2010 males constituted the victim in 10,058 of the 12,996 murder cases (77.4\%) (Federal Bureau of Investigation (FBI), 2011). While this is the case, it is important to note that the proportion of individuals murdered by an intimate partner showed different patterns across males and females (FBI, 2011). Specifically, the victim in $37.5 \%$ of the female homicides committed in the United States in 2010 was the wife or girlfriend of the perpetrator (FBI, 2011). In contrast, the victim in only $2.4 \%$ of the male homicides committed in the United States in 2010 was the husband or boyfriend 
of the perpetrator (FBI, 2011). This contrast highlights the fact that while men can also experience IPV, the impacts of victimization are particularly dramatic for women.

While death is the most extreme consequence of IPV, studies have documented a variety of physical and psychological consequences associated with IPV victimization. One large-scale study conducted by the CDCP investigating health conditions and IPV found that women who experienced IPV in their lifetime were more likely to report numerous heath conditions, such as high cholesterol, cardiovascular disease, and joint disease (CDCP, 2008). These findings are consistent with previous studies that found women who have experienced IPV in their lifetime are more likely to report a greater number of health problems, which include poor health, headaches, back pain, sexually transmitted diseases, pelvic pain, chronic pain, chronic disease, appetite loss, and digestive problems (Campbell et al., 2002; Coker et al., 2002). In terms of psychological effects, IPV victimization is associated with higher levels of depression, chronic mental illness, suicide, PTSD, alcohol abuse, and drug abuse (Coker et al., 2002; Golding, 1999). When evaluating the consequences of IPV, it is important to not only consider the direct consequences of victimization, but also the costs to society that IPV presents. One way to assess the broader consequences of IPV is to examine how IPV impacts the workplace. A review of the relevant literature conducted by Swanberg, Logan, and Macke (2005) found that IPV has important consequences not only for the victimized employee, but also for the organization as a whole. These consequences include: absenteeism, tardiness, work distraction, on the job stalking and harassment, and batterer interference with work (Swanberg et al., 2005; Reeves \& O'Leary-Kelly, 2007). Further, 
in 1995 it was estimated that 13.5 million days of work were lost due to IPV victimization (Max, Rice, Finkelstein, Bardwell \& Leadbetter, 2004). Each of these consequences can impact the individual, coworkers, and the organization as a whole (Swanberg et al., 2005; Reeves \& O’Leary-Kelly, 2007). Estimates of the economic cost of these impacts vary widely but reports have estimated that victims of IPV lose \$18 million annually and employers spend approximately $\$ 3$ to $\$ 5$ billion dollars annually as a consequence of IPV (Swanberg et al., 2005).

Costs to society can also be examined in other arenas, for example the costs associated with medical or mental health care for IPV victims. Max and colleagues (2004) examined numerous data sources (i.e., Uniform Crime Report; Medicare 5\% Standard Analytical Files) in 1995 to determine the fiscal costs of IPV in the United States. When medical costs were examined, it was estimated that IPV related assaults (both physical and sexual) cost 2.6 million dollars in 1995 (Max et al., 2004). This estimate includes visits to the emergency department, hospital stays, physician visits, dental visits, ambulance transportation, and physical therapy costs (Max et al., 2004). These medical treatments are not only costly to the individual receiving treatment, but $20 \%$ of these expenses are paid for by public sources (Max et al., 2004). In terms of mental health care, in $1995 \$ 1.4$ billion dollars was spent due to IPV victimization, with public sources paying for $18 \%$ of this cost (Max et al., 2004). Together, these estimated costs point to the far-reaching effects of IPV on those directly victimized as well as on society at large. 
Given the large number of individuals impacted by IPV and the numerous, wideranging consequences to this social problem, attempting to reduce IPV in ways that go beyond providing services to victims is necessary. Though IPV is recognized as an important issue in our society, historically much of the prevention, intervention, and research efforts have been directed at victims of IPV rather than those perpetrating abuse (Geffner \& Rosenbaum, 2001). While creating support to aid victims of IPV is both necessary and important, concentrating on victims as the focal person of interest for prevention, intervention, and research projects places the responsibility to stop violence onto the victim rather than the abuser. Thus, focusing on the perpetrator has important benefits for the prevention of IPV. These benefits are both practical and ethical, as prevention of IPV requires targeting those participating in abusive behavior and refraining from interventions that encourage victim blaming. When considering the abuser as the focal person of interest, BIPs are the most common avenue for attempting to impact the problem of IPV (Bennett \& Williams, 2001; Gondolf, 1997). These interventions were established in the 1970s and have largely taken the form of group educational programs (Dalton, 2007).

\section{Batterer Intervention Programs}

BIPs first emerged out of the social movement to stop violence against women under the premise that providing services solely for victims would not stop violence towards women. Instead, the men committing violence must be targeted for preventive intervention (Feder \& Wilson, 2005; Gondolf, 2002). Early programs utilized a feminist ideology that incorporated tactics such as peer-support to increase participants' awareness 
of power dynamics between men and women and were largely voluntary in nature (Feder $\&$ Wilson, 2005).

While BIPs targeting abusive men emerged in the 1970s, their existence has increased dramatically since the late 1980s (Gondolf, 1997). The increased prevalence of BIPs is consistent with the increased use of mandatory arrest laws for IPV incidents, increased prosecution of IPV crimes, and a greater number of convictions and guilty pleas for IPV offenders (Gondolf, 1997; Price \& Rosenbaum, 2009). As the number of individuals arrested for IPV related crimes increased, BIPs were utilized for two primary reasons. First, they were utilized due to the potential effective quality of this type of intervention. Second, they were utilized because BIPs addressed other practical issues such as prison and jail overcrowding (Gondolf, 2002). Over time BIPs have shifted from primarily voluntary attendance to mostly serving court-referred men in a communitybased rather than incarcerated setting. The fact that most states have legislation promoting the use of BIPs in sentencing and diversion speaks to their current widespread use (Price \& Rosenbaum, 2009).

While there continues to be variation in practice among programs, as the number of programs grow and time passes, programs tend to evolve to incorporate similar approaches to IPV intervention. These approaches integrate psychoeducational and/or cognitive behavioral approaches alongside the profeminist ideals with which early programs were founded (Feder \& Wilson, 2005; Price \& Rosenbaum, 2009). Currently, BIPs tend to utilize gender-specific, open-ended groups of pre-determined length (Price \& Rosenbaum, 2009; Saunders, 2008). Programs aim to provide skills training (e.g., 
tactics to prevent violence and positive relationship skills), model non-violent behavior, change thought patterns relevant to violence, provide education about sex roles, and emphasize the impact of violence on victims (Feder \& Wilson, 2005; Saunders, 2008). These goals are accomplished through lessons emphasizing behavioral strategies such as: improving communication; identifying anger cues; taking time-outs and utilizing relaxation skills; understanding what is underlying anger and the cognitions that are involved in violence; and helping men realize the costs and consequences of aggression (Rosenbaum \& Leisring, 2001).

Due to the common use of BIPs for those who have committed an IPV-related offense, examining the efficacy of these programs is imperative. Understanding the effectiveness of BIPs is important for two substantial reasons. First and foremost, the efficacy of BIPs has direct implications for victim safety. Partners of men in BIPs may feel a false sense of safety knowing that their partner is receiving intervention, which may impact their safety choices. For example, Gondolf (1988) found that women are more likely to return to their violent partners if the abuser is involved in a treatment program. If the victim in an abusive relationship believes that the BIP will be effective in changing her partner's violent behavior, she may feel it is safe to return to her partner. Therefore, an ineffective program can place a female partner in an increasingly dangerous situation (Gondolf, 1988). Second, it is important to consider how the efficacy of BIPs is related to the common use of mandated intervention. Completion of a BIP may be required for individuals involved at different levels of the criminal justice system. For example, completion of a BIP may be a requirement set forth prior to going to trial, as 
part of a plea bargain, as a sentencing condition, or as a post-release probation or parole requirement (Gondolf, 1997). This widespread use of BIPs by the criminal justice system is based at least partially on the premise that BIPs aid in preventing future offenses for individuals at each of these points within the system (Rosenbaum \& Leisring, 2001).

Despite the importance of determining program efficacy, there are many challenges in evaluating BIPs. Some of these challenges include forming working relationships with programs, determining what outcomes are considered successes, determining how long to track participants, effectively tracking participants over time, and getting honest reports about IPV from participants (Gondolf, 2002). Additionally, it can be very difficult to isolate program effects from the effects of other aspects of the criminal justice system such as arrest and monitoring (Jackson, 2003). In spite of the many difficulties researchers face when determining the success of BIPs, there have been a number of studies examining whether BIPs prevent further violent behavior toward spouses/partners.

There is a large degree of ambiguity across all studies examining BIP effectiveness in reducing IPV. Inconsistencies in the results of efficacy studies are likely due to the reasons described above, along with variations in research and evaluation design across studies (Jackson, 2003). One meta-analysis was conducted that attempted to examine varying types of efficacy studies in order to determine BIP effectiveness across studies. This meta-analysis included 22 studies of BIPs effectiveness, and across all results only a small effect of treatment was identified when controlling for the effect of being arrested (Babcock, Green \& Robie, 2004). A subsequent meta-analysis utilizing 
more stringent and conservative methods showed mixed results depending on whether the study was experimental or quasi-experimental, and whether the outcome was official reports of arrest or victim reports (Feder \& Wilson, 2005; Feder, Wilson \& Austin, 2008). This meta-analysis included ten studies and found a small treatment effect for studies that employed an experimental design and utilized official reports, but no effect when victim reports were utilized (Feder \& Wilson, 2005; Feder et al., 2008). These findings raise questions regarding the efficacy of BIPs.

While the general efficacy of BIPs in preventing further violence is unclear based on meta-analyses (Babcock et al., 2004; Feder \& Wilson, 2005; Feder et al., 2008), one comprehensive study of BIP efficacy provides promising findings for the utility of BIPs in reducing future violence (Gondolf, 2002). Through the use of a quasi-experimental design with 840 men across multiple sites, outcomes of treatment dropouts (i.e., attended the BIP for two months or less) and treatment completers (i.e., attended the BIP for more than two months) were compared (Gondolf, 2002). The findings indicate that a significantly smaller proportion of the treatment completion group re-assaulted their partners as opposed to the dropout group (Gondolf, 2002). More specifically, 36\% of those in the treatment completion group re-assaulted their partners as opposed to $55 \%$ of those in the treatment dropout group (Gondolf, 2002). This indicates that those in the treatment dropout group were 1.5 times more likely to re-assault their partners as compared to the treatment completion group (Gondolf, 2002). The findings of this study as compared to other studies of BIP effectiveness should be weighted heavily. The relative importance of these findings is based on the large sample size and use of 
numerous BIP sites. The largest study included in the meta-analysis conducted by Babcock and colleagues, beyond the Gondolf (2002) study, included 446 participants which is slightly more than half of the number of participants in the Gondolf (2002) study (Babcock et al., 2004). Additionally, the vast majority of studies included in the metaanalysis were comprised of participants from one location (Babcock et al., 2004) rather than across multiple sites. The Gondolf (2002) study contributes some support to the premise that completion of a BIP is associated with better recidivism outcomes. Taken together, the evidence regarding BIP effectiveness suggests that there may be a small positive effect for those who participate in a BIP. In addition to their possible impact on participants outcomes, the widespread use of BIPs makes them an important intervention technique to study further in order to gain a more comprehensive understanding of the role that BIPs play in reducing future violence.

Despite the variation in investigations of program efficacy, BIPs are continuously utilized by the criminal justice system. In order to create a system to judge the quality of services offered, the majority of states developed policy to regulate programs in the form of standards for practice (Geffner \& Rosenbaum, 2001; Gelles, 2001). Standards were designed to encourage uniform approaches to stop violence and prohibit the use of practices thought to be ineffective or harmful in some situations, such as couples counseling or anger-management (Bograd \& Mederos, 1999).

\section{State Standards for Practice}

State standards for BIP practice are a somewhat controversial but exceedingly common form of regulation intended to achieve quality control. The formation of state 
standards for BIP practice began in the mid-1980s after mandatory arrest laws created a context where participation in a BIP became increasingly common (Austin \& Dankwort, 1999). As the number of individuals arrested for IPV related crimes increased, so did the number of BIPs across the United States (Austin \& Dankwort, 1999). As a greater number of BIPs proliferated, it became evident that there was considerable variation in program practices. Due to inconsistent findings regarding the efficacy of BIPs in preventing further violence coupled with the large degree of variation in practices among programs, state standards became increasingly common (Bennett \& Piet, 1999).

While regulatory standards can be mandated at multiple levels of government (e.g., county or city), most standards are implemented through state-level legislation (Maiuro \& Eberle, 2008). The increasingly widespread adoption of standards is evident when you consider that the number of states utilizing standards has increased over time to include nearly all U.S. states. The most recent review of standards across the United States (Maiuro \& Eberle, 2008) determined that 45 states, including the District of Columbia, utilize some type of state standards for BIP practice as compared to 25 states in 1999 and 30 states in 2001 (Austin \& Dankwort, 1999; Maiuro, Hagar, Lin \& Olson, 2001).

The overarching goal and rationale of standards is to promote the safety of both victims and the community (Bennett \& Piet, 1999). With this goal at the forefront, standards for practice typically encompass the guidelines and protocols to which programs in a designated area are expected to adhere (Maiuro et al., 2001). While standards vary from state to state and there is no national body that provides oversight of 
standards, studies have shown that the broad components of standards tend to be similar across states (Bennett \& Vincent, 2001; Dalton, 2007; Maiuro \& Eberle, 2008). The most recent assessment of standards across the United States found that common components include: requirements addressing treatment philosophy and curriculum, length of treatment, treatment modalities, client assessment, victim contact, confidentiality of records, release of information policies, and facilitator training (Maiuro \& Eberle, 2008). In theory, creating standards that regulate these characteristics of programs will lead to the elimination of programs that use practices that cause more harm than good. Further, standards will encourage programs to change these practices, thus creating a system of quality assurance for judges, probation officers, and victims (Geffner \& Rosenbaum, 2001; Gelles, 2001).

Despite the good intentions underlying general standards and the popularity of this approach, it is important to note that there is considerable debate in the academic and practitioner communities as to whether the field of batterer intervention is ready for standards. Some reviewers claim that standards may not be as useful as anticipated due to four specific critiques. First, critics of the standards question the extent to which standards are based on scientific evidence. These reviewers claim that standards are, for the most part, not based on scientific evidence and instead are driven by advocates in the field (Austin \& Dankwort, 1999; Holtzworth-Munroe, 2001). From this point of view, standards have been and continue to be created primarily from the ideologies of those that work with battered women and common-sense best practices that are not guided by empirically validated theory or philosophy (Gelles, 2001). Second, standards may limit 
innovation in the field (Austin \& Dankwort, 1999) and prohibit practices that may be beneficial for some populations (e.g., the use of process oriented psychotherapy groups for men that display high levels of dependency) (O'Leary, Heyman \& Neidig, 1999; Saunders, 1996). Many state standards prohibit the use of specific types of intervention (e.g., couples counseling), despite evidence that alternative forms of treatment can be useful for certain populations (Holtzworth-Munroe, 2001; O'Leary et al., 1999). The creation of standards imply that there is an ideal program structure and model from which all men can benefit, yet researchers are discovering that offender subtype along with readiness for change and stage of change may profoundly impact how an individual responds to interventions (Begun, Shelley, Strodthoff \& Short, 2001; HoltzworthMunroe, 2001). Third, development of standards may limit future research that may help determine what practices are most effective (Austin \& Dankwort, 1999). As previously discussed, the efficacy of BIPs in preventing further violence is uncertain. Adopting standards that dictate practices and program characteristics may inhibit further growth and innovation in the field, as well as researchers' ability to determine the impact of novel intervention techniques (Gelles, 2001). Finally, the efficacy of standards in improving BIP outcomes and reducing recidivism is unknown (Holtzworth-Munroe, 2001). Given that the ultimate outcomes of these policies are unknown, it may be premature to dictate the ways in which programs are run. Regardless of these critiques, standards have spread to nearly every U.S. state, with Oklahoma (in 2010), Alabama and Nebraska (in 2008) most recently passing laws to adopt some form of standards for BIP practice. 
Although 45 states including the District of Columbia have standards (Maiuro \& Eberle, 2008), requirements surrounding compliance and the extent to which monitoring and enforcement of standards occur vary widely (Tolman, 2001). According to a review of state standards conducted in 1997, $73 \%$ of the 37 states with standards at that time indicated that some type of monitoring process should take place, but very few described the process by which monitoring would occur (Austin \& Dankwort, 1999). Further, upon interviewing programs, Austin and Dankwort (1999) found that very few programs were actually monitored to ensure compliance.

More recently, the administrative bodies for state standards were examined across the United States. This investigation indicates that there are differences across states in the agency or body that provides oversight to ensure standards are being met (Maiuro \& Eberle, 2008). Specifically, some states utilize committees comprised of individuals relevant to the IPV collaborative response (e.g., victim advocates, judges, providers, etc.) and others rely on a single organization (e.g., victim advocacy agency or administrative body) to oversee compliance with standards (Maiuro \& Eberle, 2008). While the type of administrative body for standards was examined in this study, the degree to which standards were monitored and enforced by these bodies was not fully explored. Currently, the exact number of states with different degrees of enforcement remains unknown. All that can be said is variation does exist with some states requiring compliance through formal monitoring or certification of BIPs (e.g., Washington, Kentucky, Colorado, and Virginia) and other states creating standards without a formal monitoring system in place (e.g., Oregon). 
Oregon joined the majority of states with standards in 2006, when Oregon Administrative Rules (OAR) regulating BIPs were passed by the state legislature (see Appendix A). Administrative rules include regulations for agencies that are approved through state legislature. Administrative rules describe requirements that a given agency is expected to follow and these requirements are to be administered and followed as would a law (Diver, 1983; Fuchs, 1938). While Administrative Rules are thought of as laws, one critique of Administrative Rules is that they can be written in ways that allow more or less flexibility (Diver, 1983). The Administrative Rules for BIPs in Oregon, or the state standards, are aimed at creating regulation for BIPs working with abusive men in heterosexual relationships (Oregon Department of Justice (ODOJ, 2009). In line with the notion of flexibility above, some of the aspects of the rules are written as requirements (e.g., program length) and others are written as recommendations or program characteristics to include when possible or needed (e.g., cultural relevance and mixed gender co-facilitation). Similar to the content areas included in standards across the United States, the Oregon standards address many aspects of program functioning such as intake procedures, information release, victim contact, and facilitation strategies. Additionally, they require that BIPs utilize "appropriate" intervention strategies, establish duration of interventions, and specify training for staff (ODOJ, 2009). Like other states, there is currently no statewide and consistent monitoring or enforcement system to ensure that the standards are successfully implemented. The lack of monitoring and enforcement in the state of Oregon begs the question as to whether or not BIPs are actually implementing the criteria of the standards into their program practices and procedures. 
Without information as to whether or not standards are implemented, it remains difficult to determine whether state standards achieve their intended purpose (e.g., increased victim safety and quality assurance). Thus, in order to develop a comprehensive understanding of the extent to which standards have been implemented in Oregon, an examination of the theoretical framework of policy implementation is useful.

\section{Policy Implementation}

In order to understand organizational and individual outcomes, it is important to have a clear conceptualization of the larger context that the organizational and individual outcomes are situated within. The ecological model is one way to conceptualize the ways in which social and political context, in the form of state policy, may impact outcomes of interest (Bronfenbrenner \& Morris, 1998). Applied to BIPs, this model asserts that in order to understand the ways in which individual outcomes within a BIP occur one must also be aware of the larger contextual factors at play, such as state policy for BIP practice (see Figure 1). Taken a step further, not only is an understanding of the content of standards necessary, but knowledge as to whether or not programs actually implement the policy is vital to developing a full appreciation of the context that individual outcomes are happening within.

Studies of policy implementation are needed to determine whether and how social policy affects both organizational and individual behaviors. The study of policy implementation gained popularity in the 1970s as a result of increased need to understand the effects of social policy enacted in the Great Society legislation (McLaughlin, 1987; O’Toole, 2000). Around this time, researchers and policy analysts realized that 
examining policy failure in terms of the content of policies was not sufficient (Barrett, 2004; McLaughlin, 1987). The field began to appreciate that understanding policy success required some understanding of the mechanisms of implementation (Elmore, 1979). Given this, researchers moved away from the notion that the creation of a sound social policy was enough to ensure that the components of the social policy would be successfully enacted. Instead, studying the importance of implementation gained popularity (Barrett, 2004). Some writers have noted a decline in policy implementation research for policy analysts since the 1990s (e.g., Barrett, 2004; O'Toole, 2000; Saetren, 2005). However, a recent study examining the number of policy implementation studies that have been published across many fields found that the number of policy implementation related publications has grown throughout the 1990s and 2000s (Saetren, 2005). The author of this review also noted that the diversity of fields that publish implementation studies has increased (Saetren, 2005). The study of policy implementation has become common not only in policy specific fields, but also in other areas such as health, education, law, environment, and economics (Saetren, 2005). Thus, the field of policy implementation has been and continues to be an active area of interest. Perhaps in part due to the wide variety of disciplines conducting policy implementation studies, there have been discrepancies in how the field is defined and conceptualized. Definitions of policy implementation vary. Some definitions of implementation include the entire process from creation of policy to impact in the real world. Others restrict the definition to just the actions of those responsible for enacting a policy (O'Toole, 1986). For the purposes of this study, the broadest definition is utilized. 
Specifically, policy implementation occurs when an authoritative decision or intention is carried out to have an impact on the world (Berman, 1978; O'Toole, 2000). Policy implementation has come to be understood as a multi-actor process that typically takes place at multiple levels and across multiple organizations (O'Toole, 1986; 2000). The study of this process is labeled policy implementation analysis and aims to understand why authoritative decisions do or do not lead to expected results at the level of the individual or the organization (Berman, 1978). The authoritative decisions that Berman (1978) is referring to and are captured in policy implementation analysis include policies, plans, and laws.

While the study of policy implementation has become more common since the 1970s, the field has yet to come to agreement about crucial aspects of the field, including critical variables to consider, definitions of success, and the appropriate theoretical framework (O'Toole, 1986; 2000). While there is diversity in the field as to defining success and determining which variables indicate success, a review of approximately 100 studies considering implementation indicates that commonly examined variables include: policy characteristics; resources; implementation structure; the number of individuals responsible for implementation; attitudes and perceptions of implementing personnel towards the policy and its feasibility; alignment of clientele with the content and purpose of the policy; and timing of the policy relative to the political and economic climate (O'Toole, 1986). In a more recent review, O'Toole (2000) found that little has changed in terms of commonly used explanatory variables. Further, an even more recent review reveals that the number and breadth of explanatory variables is growing (O'Toole, 2004). 
These variables are used to provide information and context relevant to the success of implementation.

Creating a consistent definition of success has been extremely difficult in the field of policy implementation analysis due to the fluidity of policy implementation (McLaughlin, 1987). The process by which policies are implemented is typically nonlinear due to changes in the emphasis on implementation over time because of factors such as budget, administrative attention and support, individual interest, motivation, and involvement (McLaughlin, 1987; Saetren, 2005). Thus defining and examining success at any point in time may yield results that are extremely dependent on the current atmosphere and context. Despite this, some more prevalent definitions of successful implementation include aspects of coordination, speed, consistency, participation, localism, diversity and access (O'Toole, 1986). It is evident that when examining the more common gauges of success, some of these definitions contradict one another (e.g., consistency and localism). Thus, determining success appears to be contingent on the content, context, and goals of the policy.

Not only are the variables of interest and definitions of success varied, but the theoretical framework from which policy implementation researchers conduct research differ. Theorists have described two frameworks of implementation analysis: top-down and bottom-up (Barrett, 2004; Berman, 1974; Elmore, 1979; Matland, 1995; O’Toole, 1986; 2004). Early on, these frameworks were a source of debate and contention in the field, but have since come to be appreciated as complementary rather than contradictory (O'Toole, 2000; 2004). Current thinking in the field indicates that an understanding and 
synthesis of both perspectives will yield the most valid and practically useful research (O’Toole, 2004).

The top-down framework is an approach by which implementation is studied by examining how a legislative body creates a policy that is then passed down to subsequent levels in a hierarchical fashion (Barrett, 2004; Elmore, 1979; Matland, 1995). This framework places control and responsibility at the highest level and assumes the ability to influence levels beneath (Barrett, 2004; Elmore, 1979; Matland, 1995). This framework attributes difficulties with implementation to unclear policies, policies that require too much change, too many actors being involved in the implementation process, and differences between policy and organizational values (Barrett, 2004; Matland, 1995; O'Toole, 1986). While this is a common method of policy implementation analysis, there is a major limitation to this approach. This approach assumes those making policy decisions at the highest level can actually control implementation at the lower levels, which is difficult to guarantee in the real world because of the complex systems in which policy changes are executed (Elmore, 1979; Matland, 1995).

The bottom-up analysis framework is an approach that begins at the lowest level of the implementation process. It focuses on how target populations and those delivering services implement policy as the immediate concern (Matland, 1995). This framework can be further differentiated into macro- and micro-implementation to understand how successful implementation occurs. Macro-implementation takes place when the highest body (e.g., federal or state government) creates a policy in a way that encourages the systems below it to execute the policy. The process of macro-implementation is unique 
from the process of top-down implementation described above because this process in and of itself cannot happen in isolation. Next, the process of micro-implementation is necessary where the lower organizations (e.g., service providers) must develop and carry out a plan to change their own internal processes to align with the policy (Berman, 1978). The micro-implementation process involves ongoing conversation and bargaining among stakeholders; thus, there is no finite endpoint, and the content of the policy can always be revisited (Elmore, 1979; McLaughlin, 1987). This framework asserts that most implementation issues stem from a disconnection between the macro- and micro- aspects of implementation (Berman, 1978; Matland, 1995).

One way bottom-up policy implementation is accomplished is through the formation of a council or coalition composed of individuals who will be affected by the policy. A coalition works together to identify aspects of programming that could feasibly be altered to affect the social problem at hand (Elmore, 1979). The use of coalitions allows those making organizational changes to bargain with one another and policymakers to determine how to best target the problem of interest in the form of policy.

Importantly, the formation of councils or coalitions is well known in the context of IPV prevention and intervention. The use of a collaborative response to IPV was introduced in the early 1980s through the efforts of the Domestic Abuse Intervention Project (DAIP) in Duluth, Minnesota (Shepard, Falk \& Elliot, 2002). The DAIP attempted to integrate the IPV prevention and intervention efforts of numerous stakeholders, including police, judges, victim services, and BIPs (Shepard et al., 2002). 
This collaborative response has been and continues to be advocated in the context of IPV because coordination should decrease fragmentation of the key agencies involved in addressing IPV and invite an increasingly cohesive response to this social problem (Hart, 1995). The efforts of the DAIP were successful in that, a community collaborative response to IPV is currently an exceedingly common component of state standards (Austin \& Dankwort, 1999).

Community collaboration in the context of IPV can include a number of different stakeholders. Agencies that are typically involved in a coordinated response to IPV include police, prosecutors, judges, probation officers, BIPs, battered women's services, and battered women's advocates (Mederos \& Perilla, 2004). Some models of community collaboration extend to include additional stakeholders, such as healthcare providers, drug and alcohol services, religious organizations, and child welfare agencies (Clark, Burt, Schulte \& Maguire, 1996). Each agency involved in the collaborative response is responsible not only for their piece of the intervention process, but also is expected to communicate with other relevant agencies.

The integration of these community agencies is sometimes described as a domestic violence council and may include some or all of the partners described above. Allen (2006) found these types of councils can potentially play an important role in creating a coordinated response within the community, though the impact of the councils largely depends on factors such as creating a shared mission and effectively navigating power differences among community partners. Theoretically, prevention and intervention will be more successful if the entire community is held responsible for holding 
perpetrators accountable and ensuring victim safety, rather than agencies individually (Klevens, Baker, Shelley \& Ingram, 2008; Shepard et al., 2002).

This sentiment is reflected in the purposes of the Oregon state standards. The standards assert two specific purposes: "To foster local and statewide communication and interaction between BIPs and victim advocacy programs, and among BIPs; and to help ensure that BIPs operate as an integrated part of the wider community response to battering" (ODOJ, 2009, p. 1). The BIP standards in Oregon recommend that programs have regular contact with victim advocates, the criminal justice system, other BIPs, and related social services, including a domestic violence council if one exists in the area (ODOJ, 2009). Community collaboration is a key area of focus that BIP standards target for development.

This contextual history of collaboration and councils among differing stakeholders in the field of IPV intervention creates an environment where bottom-up policy implementation may be successful. Nonetheless, there is one drawback of this approach that is important to recognize. This drawback stems from the fact that while bottom-up implementation takes organizational capacity into account and integrates views of multiple stakeholders, the standards of success are conditional for each organization and change over time. When this implementation approach is utilized, each organization is permitted to define success individually and this definition might shift as the climate and culture of the organization evolves. Thus, this approach may make uniformly determining compliance with the policy difficult (Elmore, 1979). 
Policy implementation theorists advocate for and have attempted integration of the top-down and bottom-up frameworks with limited success (Matland, 1995; Saetren, 2005). These integrations take two major forms: the first combines the two frameworks; the second describes when each of these frameworks is most appropriate (Saetren, 2005). Recent theory on this topic takes into account how both perspectives can be useful. Matland (1995) posits that the distinctions between these frameworks may be the result of the types of policies studied- clear and simple policies being best suited for top-down analysis and more ambiguous policies being best suited for bottom-up approaches. Further, O'Toole (2000) suggests that while understanding who is responsible for creating and carrying out a given policy is important, understanding the issues and experiences at both the top and bottom levels are necessary to fully understand policy implementation.

\section{Implementation of BIP Standards in Oregon}

The integration of both a top-down and bottom-up policy implementation theoretical framework can be directly applied to the introduction of state standards for BIPs in Oregon. Like the majority of states in the U.S., the state of Oregon adopted a set of standards for BIPs. In 2002, the Oregon state legislature passed Senate Bill 81 (SB 81), which required the development of state standards for BIP practice. The mandate for state standards introduced at the state level and requiring local and organizational change from key program staff members is an example of how state standards for BIPs can be thought of as a top-down process of policy implementation. Specifically, the requirement that state standards for all BIPs in Oregon be developed allows for an investigation into 
overall compliance with the policy across programs. While the top-down approach is useful, it is important to recognize that once the mandate for standards was made, the way in which their content was developed, which included input from BIP staff, victim advocates, and probation officers, clearly demonstrates aspects of a bottom-up approach for those involved in the development of the standards.

In accordance with SB 81, the state of Oregon began creating a set of standards in 2002 with the use of a diverse committee of individuals that would be affected by the standards known as the Oregon Attorney General's BIP Standards Advisory Committee (Standards Advisory Committee). This committee was and continues to be composed of individuals from various groups and agencies related to IPV intervention and prevention in the state of Oregon. The current committee includes: government officials working in positions related to IPV prevention, attorneys, batterer intervention providers, victim advocates, community corrections officers, one judge, one IPV coalition member, and one university professor. Various forms of this committee have been meeting since 2002, when SB 81 was passed mandating that standards be developed for BIPs. The committee worked together to develop the standards that were finalized in 2006 and are still being used today. Consistent with the coalition and bargaining notions discussed above, the committee continues to meet several times per year to discuss, refine, and adjust standards as necessary, in addition to providing education about the standards and responding to stakeholder feedback. It is important to note that while the Standards Advisory Committee is permitted to suggest alterations to the standards as they see fit, only one substantive change has been made since their original development. This change 
was enacted in September 2012 and was concentrated on the reduction of program length. The use of a committee composed of those to be affected by the state standards in their creation is consistent with bottom-up policy implementation as those who were going to be impacted by the policy were and continue to be involved in developing the content and scope of the standards.

One important caveat in the use of the bottom-up framework in this context is that BIP staff members may have experienced the introduction of standards differently depending on the extent to which they contributed to the creation of the standards. Specifically, the bottom-up framework may be appropriate for those staff members who participated in developing the standards, as well as staff members who have a clear understanding of how the committee functions and how the standards developed. Still, this framework may not be as relevant for those who were not a part of or knowledgeable about the standards' development as they were likely not involved in the bargaining and coalition activities inherent in the process of bottom-up policy implementation.

Understanding the process of implementation overall in the context of compliance, as well as experientially from a diverse group of program staff members, will allow an exploration into a synthesized framework as advocated by O’Toole (2004).

\section{Applied Social Psychology and Policy Implementation}

The study of policy implementation is highly contextual due to the unique background and circumstances inherent in each policy (McLaughlin, 1987; Saetren, 2005). Thus, examining policy implementation exclusively for universal laws or basic processes may fail to account for the unique qualities of each individual policy and the 
environment in which it is enacted. Additionally, due to the diverse content of social policies, understanding the way in which each policy is implemented requires knowledge from multiple disciplines in order to comprehend the rationale and impact of the policy, as well as processes that may impact implementation.

One discipline that may be particularly valuable in developing an understanding of policy formation, implementation, and adherence is that of psychology. The collaboration of policymakers and psychologists has the potential to be immensely useful in creating policies that take the complexities of people into account (Esses \& Dovidio, 2011; Fischhoff, 1990). Psychologists can contribute useful information regarding the ways in which people might perceive and react to various policies, as well as likely outcomes of different policies (Esses \& Dovidio, 2011; Fischhoff, 1990). In particular, because the policy process is a highly social (Bauer, 1965), social psychologists with pertinent expertise regarding the various social psychological processes that may impact the process of implementing a policy can be especially valuable.

Further, the study of policy implementation is highly applied as the findings can be directly utilized by policymakers and/or community members to better understand why a policy has or has not been successfully implemented. Those familiar with applied social psychology and its focus on socially relevant and useful research are well positioned to contribute to the understanding of the policy process. Together, these factors support the use of applied social psychology to better understand the process of policy implementation. In order to draw on the strengths of applied social psychological theory as it applies to policy implementation, the constructs of perceived control, 
rationalization (i.e., one potential explanation for change in negative attitudes towards the standards), reactance (i.e., one potential explanation for maintenance of negative attitudes towards the standards), absoluteness, and legitimacy will be assessed.

Actual control and perceived control. The policy implementation literature is helpful in identifying a framework from which the process of implementation can be understood. However, aspects of social psychology may contribute one way to make sense of the underlying interactional processes that might impact how policy is implemented and the extent to which it is successful. This is especially true when thinking about the differing levels of involvement key program staff members potentially had or perceived having in the development of the standards. Understanding the amount of control key program staff members perceive having over the content and scope of the standards, or as it is known in the psychological literature, perceived control, may be vital to comprehending the extent to which they implemented the standards and how they did so.

In psychological study there is ambiguity, confusion, and difficulty navigating the concept of control due to the inconsistent definitions and uses of terms associated with control (Rodin, 1990; Skinner, 1996). The overarching construct of perceived control is comprised of four theoretical frameworks: locus of control, causal attributions, learned helplessness, and self-efficacy (Skinner, 1995). Together these frameworks integrate the influence of personality, cognitions, and motivation into a comprehensive conceptualization of perceived control (Zimmerman \& Zahniser, 1991). While these frameworks are integrated into the notion of perceived control, it is important to note that 
these frameworks individually are often times used synonymously with the term perceived control (Skinner, 1996). The ambiguity in the definition of perceived control stems not only from the combination of these theoretical frameworks, but also from the acknowledgement that perceived control does not remain constant and instead varies based on situation (Bandura, 1982; Paulhus \& Christie, 1981; Zimmerman \& Zahniser, 1991).

While multiple definitions for control exist, the concept of perceived control described by Baron and Rodin (1978) and Rodin (1990) provides one lens that may be helpful in understanding the extent to which policy implementation is successful. This conceptualization of perceived control does not focus on one's ability to enact a desired outcome. It focuses instead on one's ability to meaningfully contribute to decisionmaking processes that will subsequently affect one's self (Rodin, 1990). This view of perceived control highlights the importance of the extent to which individuals perceive they have some control over the process (Rodin, 1990). This notion of perceived control asserts that the more individuals are integrated into the policy creation process, the more aligned they will feel with the policy. This will in turn affect the extent to which they make efforts to implement and adhere to the policy.

Definitions of perceived control that focus on individual control over a given action have origins in the self-efficacy and learned helplessness literature. Conversely, the conceptualization of perceived control as the extent to which one perceives to have influence over the policies that will ultimately affect them can be traced back to the concept of perceived control as sphere specific (Paulhus \& Christie, 1981). The 
understanding of perceived control as dependent on context developed by Paulus and Christie (1981) has its roots in the study of external versus internal locus of control (Paulhus \& Christie, 1981; Skinner, 1995). The locus of control construct was introduced by Rotter (1966) as a conceptualization of the degree to which individuals perceive control over their lives. When examining locus of control, individuals may perceive experiences of control on two ends of a single continuum, internal versus external (Rotter, 1966; Skinner, 1995). Individuals who perceive outcomes as contingent on their own behaviors are said to have an internal locus of control, while individuals who perceive outcomes as not contingent on their own behaviors are said to have an external locus of control (Rotter, 1966). Locus of control is not a constant trait; it is possible for individuals to experience an internal or external locus of control across different experiences. Further, the locus of control construct can be thought of as a continuum where individuals can perceive different levels of locus of control rather than just the two bipolar endpoints. Locus of control has been examined in numerous contexts and has come to be a popular construct in the psychological literature (Rothbaum, Weisz \& Snyder, 1982). For example, locus of control has been and continues to be studied in numerous areas contexts such as health (e.g., Farone, Fitzpatrick \& Bushfield, 2008; O’Hea et al., 2009), the workplace (e.g., Wang, Bowling, \& Eschleman, 2010; Wang, Tomlinson \& Noe, 2010; Kish-Gephart, Harrison \& Treviño, 2010), and education (e.g., Flouri, 2006; Gifford, Briceño-Perriott \& Mianzo, 2006; Mavropoulou \& Padeliadu, 2002). 
As the study of locus of control progressed, researchers and theorists recognized that to fully understand the impacts of this phenomenon it is important to appreciate the context of the realm in which the perceptions and outcomes occur. This history has led to one conceptualization of perceived control, which focuses on context and may be particularly relevant to the study of perceived control over policy. Specifically, when examining the effects of perceived control on a given outcome, differentiating whether the control is in sphere of personal control, interpersonal control, or sociopolitical control is vital (Paulhus \& Christie, 1981; Paulhus, 1983). Perceived control in the context of personal control focuses on individuals' innate ability to achieve whatever task they are attempting to accomplish. This conceptualization of perceived control has also been termed personal efficacy (Paulhus \& Christie, 1981). High personal control is based on the individual perceiving they have the internal skills, such as intelligence or athleticism, necessary to successfully navigate a nonsocial task (Paulhus, 1983; Zimmerman \& Zahniser, 1991). Interpersonal control focuses on the extent to which an individual feels they have control over interactions with others. This may include one's perceived ability to successfully work in groups towards a goal, as well as to develop and maintain relationships both inside and outside of the family unit (Paulhus, 1983; Zimmerman \& Zahniser, 1991). Sociopolitical control refers to one's perceived ability to successfully navigate and impact social and political systems. This may include the extent to which one feels they can impact policy decisions or organize with others to make their opinions about a given policy known (Paulhus, 1983; Zimmerman \& Zahniser, 1991). The separation of these spheres of control has been helpful in differentiating the construct to 
allow for a deeper theoretical understanding of perceived control, as well as advances in measurement. This is especially relevant in the sociopolitical realm, as many traditional measures of perceived control have resulted in confusing and unexpected results when applied to policy relevant contexts (Paulhus, 1983; Zimmerman \& Zahniser, 1991).

The Spheres of Control Scale or SOC Scale was developed in response to the limited success of unidimensional measures of control in examining perceived control across contexts. The SOC Scale was originally developed in 1981 under the premise that personal control, interpersonal control, and sociopolitical control are conceptually unique and distinct (Paulhus, 1983; Paulhus \& Christie, 1981; Spittal, Siegert, McClure \& Walkey, 2002). The creation of the SOC was largely driven by the desire to capture the multidimensionality of the construct of control that had not yet been captured in previous measures. Specifically, previous measures of control (e.g., Rotter, 1966) had limited success accounting for variability in assessments of control due to the unidimensionality of the measures (Paulhus \& Christie, 1981). Given this inadequacy, Paulhus and Christie (1981) aimed to create a measure that examined the experience of control in three distinct arenas under the assumption that individuals may have differing experiences of control that are dependent on context.

The utility of examining these three dimensions of perceived control has been supported empirically. When items corresponding to all three types of perceived control were used simultaneously to create a one factor model of perceived control the model fit was significantly worse than that of the three factor model (Paulhus, 1983). Thus, the distinction of these three types of perceived control is both conceptually and empirically 
viable. Since the initial development of the SOC scale, the item content and measurement structure has been continually refined and the scale is currently in its third edition (Paulhus \& Van Selst, 1990). Studies of the measurement structure of the third edition of the SOC have not supported the use of the SOC-3 scale over the SOC-1 due to inadequate model fit for the factors of personal control and interpersonal control (Spittal et al., 2002). While this is the case, as with the first edition, the items from the sociopolitical scale included in the SOC-3 grouped together as expected (Spittal et al., 2002).

When attempting to understand the degree of perceived control one has over the introduction of a new policy, the distinction between types of perceived control is important to consider. In this context, the conceptualization of sociopolitical control is most relevant. This dimension of perceived control focuses on the extent to which an individual perceives that they may be able to impact policy and unite with others to make their voices heard. In order to assess perceived sociopolitical control, one subscale of the third edition of the SOC (Paulhus \& Van Selst, 1990) may be useful in developing interview questions that assess experiences of sociopolitical control among key program staff members. This subscale includes ten items that attempt to understand the extent to which individuals perceive control over their social and political context. Examples of items utilized by this subscale include: By taking an active part in political and social affairs we, the people, can control world events; The average citizen can have an influence on government decisions; With enough effort we can wipe out political corruption; and, It is difficult for us to have much control over the things politicians do in office (Paulhus \& Van Selst, 1990). While these example items can inform the 
development of interview questions relevant to key program staff members' experiences with implementation, other items from the scale are less applicable. For instance, some items are explicitly focused on specific aspects of the social and political sphere such as war, economics, and large corporations (Paulhus \& Van Selst, 1990). Items that focus on these areas are less informative as to understanding sociopolitical control in the context of key BIP staff members and the implementation of state standards.

Perhaps due in part to the questionable measurement structure of the SOC, along with the desire to examine sociopolitical control as a component of empowerment, Zimmerman and Zahniser (1991) built upon the SOC to create the Sociopolitical Control Scale or SPCS. The SPCS is comprised of 17 items that assess leadership competence and policy control (Zimmerman \& Zahnsier, 1991). This scale of sociopolitical control has been examined in numerous contexts and is commonly integrated into studies of empowerment. For example, the SPCS has been utilized in studies of policy activist groups (Itzhaky \& York, 2000), resiliency (Zimmerman, Ramirez-Valles \& Maton, 1999), youth participation in anti-tobacco campaigns (Holden, Crankshaw, Nimsch, Hinnant \& Hund, 2004), and involvement with neighborhood associations (Ohmer, 2008). While the research examining the sociopolitical subscale of the SOC and its relationship to outcomes of interest is limited, there has been more research examining the SPCS as it relates to outcomes. For example, the SPCS has been shown to be related to citizen participation (Ohmer, 2008), increased activist experience (Itzaky \& York, 2000), and leadership, engagement and encouraging behaviors in an anti-smoking campaign (Holden et al., 2004). Thus, it appears that increased sociopolitical control as 
measured by the SPCS is associated with positive outcomes in diverse settings relevant to the sociopolitical sphere.

While the entire scale has been incorporated into diverse studies, only one of the two parts of the SPCS is relevant to the proposed study. This portion includes the items that assess policy control, or the extent to which one feels they have control over policies that may impact them. Items assessing policy control include: There are plenty of ways for people like me to have a say in what our government does; Most public officials wouldn't listen to me no matter what I did; I enjoy political participation because I want to have as much say in running government as possible; and I feel like I have a pretty good understanding of the important political issues which confront our society (Zimmerman \& Zahniser, 1991). When comparing the items relevant to policy control to the sociopolitical items from the SOC it is evident that there are many commonalities. Both scales attempt to gauge whether or not the individual perceives the ability to effect policy and the actions of those in political power. Thus, information from both scales may be useful in developing qualitative interview questions to aid in determining the extent to which BIP key program staff members perceive sociopolitical control.

In order to integrate the relevant sociopolitical control items from the SOC-3 and SPSC into a qualitative interview format, some shifting of wording occurred because both scales are designed to be utilized with a Likert scale response format. After modification of the wording to specify context and allow an open response format, the following questions were included to assess perceived sociopolitical control:

- Can you describe how you feel about the creation of state standards? What about 
the people involved in this process?

- What are your thoughts about creating state policy to influence program practices?

- Do you believe that the individuals who participate on the Standards Advisory Committee represent most providers? Why do you think this?

- To what extent do you feel that you can influence the content or scope of the standards?

- If you had wanted to participate in the creation of standards, so you think you would have been able to do so?

- If you wanted to see something changed in the standards to what extent do you believe you could influence those changes?

In addition to assessing perceptions regarding sociopolitical control, it may also be important to gain information regarding participation or actual control over the policy of state standards. Theorists and researchers have suggested that the extent to which an individual has actual control over their behaviors may be useful to consider (Ajzen \& Madden, 1986; Wortman, 1975). Having actual control indicates that the individual has a higher degree of means and facilitators to achieve the desired outcome as compared to someone that lacks actual control (Ajzen \& Madden, 1986). Studies in the sociopolitical realm have found that participating in organizations that aim to shape policy, an action that can be conceived as actual control, predict experiences of perceived control (Becker, Israel, Schulz, Parker \& Klem, 2002; Kieffer, 1984; Schulz, Israel, Zimmerman \& Checkoway, 1994). These studies suggest that in the context of BIP key program staff 
members and state standards, those that were able to actively contribute to the policy creation process may develop a higher sense of perceived control over the standards. Thus, to complement the questions described above to fully appreciate key program staff members' perceptions of control, as well as the extent to which actual control is important, the following question were included in the interview guide:

- What was your role in creating the Oregon BIP Standards? Did you play a part in their development? Are you aware of the process by which the standards were developed? Can you describe the process of creation, as you understand it? When applying this view of perceived control to the context of BIP standards, several predictions can be made based on the association between perceived sociopolitical control and positive outcomes (Holden et al., 2004; Itzhaky \& York, 2000; Ohmer, 2008). First, using the logic of the association between actual control and perceived control, it is expected that key program staff members involved in the development of the standards will perceive more control over the standards than those that did not participate in the creation of the standards. Second, since perceived control should be associated with positive outcomes regarding standards, it is expected that key program staff members reporting high compliance will perceive they are or were involved with the process of creating the standards. Third, the rationale of perceived control can be can be extended to predict that key program staff members who believe they can influence the standards if that is a goal of theirs will be more aligned with the standards and more compliant with the standards than those who do not feel they can impact the standards. Thus, gaining a comprehensive understanding of the role that key 
program staff members played in the development of standards, as well as the extent to which they are aware of the process by which standards were created and how much control they feel they have over that process, may help explain their views towards the standards. It may also help explain the extent to which their program is in compliance with the standards.

Rationalization, reactance, and absoluteness. While the notion of perceived control is relevant, understanding individual's reactions to the introduction of a new policy may provide valuable insight necessary to better understand which BIPs implement state standards and the processes by which they did so. As is found in the policy implementation literature, the perceptions of those expected to ensure the policy is implemented (i.e., program directors) may play a crucial role in whether or not implementation successfully occurs. In order to understand how a policy is perceived, it is necessary to determine how the policy was introduced. Laurin, Kay, and Fitzsimons (2012) offer a helpful analysis of two possible reactions to the introduction of new policy and one way that these divergent reactions can be combined into a coherent framework. Specifically, Laurin and colleagues (2012) suggest that while the traditional social psychological literature proposes two divergent outcomes, rationalization and reactance, to the introduction of a new policy that reduces independence to make decisions, the degree to which the policy is definite may differentiate why these opposing outcomes occur. Each of these processes, as well as the way in which Laurin and colleagues (2012) integrate them, is described below. 
Rationalization. In order to gain a full appreciation for the process of rationalization and the ways in which this process may impact reactions to the introduction of policy that limits freedoms, it is first necessary to develop an understanding of the origins of this construct. The origins of rationalization can be traced to the theory of cognitive dissonance. The theory of cognitive dissonance was developed by Leon Festinger (1957) more than five decades ago. Since the theory was introduced, it has proven to be of the most influential theories in the field of social psychology (Nail \& Boniecki, 2011; Thibodeau \& Aronson, 1992). The volume of studies, which includes more than one thousand investigations, conducted to explore this theory speaks to its influence in the field (Thibodeau \& Aronson, 1992). Studies of cognitive dissonance span many contexts including work productivity and wages (Adams \& Rosenbaum, 1962), water conservation (Dickerson, Thibodeau, Aronson \& Miller, 1992), consumer behaviors (Cummings \& Venkatesan, 1976), and group interactions (Matz \& Wood, 2005). While the study of cognitive dissonance has been and continues to be an active area of inquiry, some have noted that the study of this phenomenon, and others like it, has been limited due to difficulties in measurement and replication (Cialdini, Trost \& Newsom, 1995). The nature of these difficulties becomes apparent when the construct of cognitive dissonance is defined.

The theory of cognitive dissonance proposes that individuals strive for consistency among attitudes and between attitudes and behavior (Festinger, 1957). Individuals have numerous cognitions that may coincide with one another, may be irrelevant to one another, or may conflict (Festinger, 1957). Various cognitions are 
associated with congruous and incongruous behaviors and individuals strive for concordance between their cognitions and behaviors (Festinger, 1957). For example, individuals who identify as BIP directors and view their power in decision making as important may experience dissonance when the introduction of state standards mandated that they make certain decisions regarding their program. The experience of dissonance, such as the one described above, leads to discomfort and motivates individuals to alter their cognitions or behaviors in order to achieve consistency (Festinger, 1957). For example, a program director may feel that he or she has training and experience sufficient to determine the appropriate length of intervention for various individuals and he or she may experience discomfort when the standards, which include requirements relevant to program length, were introduced. In the face of this discomfort, the individual is motivated to align his or her thoughts about determining program length and their behaviors. If the individual complies with the requirements of the standards, he or she individually may come to view the length requirement as appropriate in order to coincide with the length requirement included in the standards.

It is important to note that the experience of dissonance is not an all or nothing phenomenon. Rather, factors such as the importance of the dissonant elements and the proportion of dissonant elements as compared to consonant elements impact the degree to which an individual feels the negative effects of cognitive dissonance (Festinger, 1957). Further, factors such as the degree to which the individual feels pressure to take part in the dissonant behavior, as well as the extent to which the individual has anticipated the dissonant experience, may impact whether or not and the extent to which cognitive 
dissonance is experienced (Festinger, 1957; Festinger \& Carlsmith, 1959; Kay, Jimenez $\&$ Jost, 2002). These caveats are especially important when considering the introduction of policy, such as the state standards. If individuals feel a high degree of pressure to comply with the standards, or if they were able to anticipate the introduction of the standards, they may not experience the same degree of dissonance that an individual who does not feel pressure to comply or was not able to anticipate the standards would experience.

One possible reaction to the experience of cognitive dissonance is rationalization or the attempt to construct an explanation for the experience of dissonance (Kay et al., 2002). Rationalization allows individuals to adapt their cognitions to become more synchronous with the cognition or behavior that is causing dissonance and distress, thus decreasing the aversive effects. The process of rationalization can be used to provide consistency in cognitions and behaviors in the case of voluntary and non-voluntary behaviors. For instance, one voluntary behavior that the process of rationalization has been applied to is the study of alcoholism. Studies of individuals with alcohol dependence have found that rationalization of problematic drinking occurs under the pretense that there is conflict between what the individual knows about the dangers associated with alcohol abuse and their behaviors, in other words, there is dissonance between their attitudes and behaviors (Chai \& Cho, 2011; Jellinek, 1946; Ward \& Rothaus, 1991). In this context the rationalization is typically a way to change cognitions in order to continue the behavior. For instance, individuals may point out the positive aspects of drinking, such as enjoyment and opportunities for socialization, in order to 
rationalize their choice to continue drinking. It is important to note that changing cognitions to coincide with behaviors is possible in situations where changing behavior is voluntary, but there are also situations in which the choice to change behaviors is not voluntary.

While studies of rationalization in the context of voluntary behavior are informative, when attempting to understand reactions to the introduction of policy it is important to consider situations in which behavioral change is not voluntary. The process of rationalization to the introduction of a new policy that requires certain behaviors, whether it is issued through government or an organization, is not uncommon. As policies shift, individuals often alter their perceptions and cognitions to align with the changes that will ultimately guide their behaviors (Gilbert, Pinel, Wilson, Blumberg \& Wheatley, 1998). Rather than rationalization of a behavior that runs counter to cognition, in the case of policy, rationalization is used to change cognition to coincide with required behavior change. This process has been studied in numerous sociopolitical contexts including desirability of legislation (Granberg \& Brent, 1983), voting behaviors (Beasley \& Joslyn, 2001), and university policy changes (Kay et al., 2002). These studies indicate that when unavoidable outcomes occur, such as the results of an election, individuals rationalize by changing cognitions to be more congruous with the less favorable outcome. In line with the theory of cognitive dissonance, this process of rationalization will make the previously unwanted policy appear more attractive. Thus, individuals find themselves in situations in which independence or personal freedoms are limited by policy, they would be motivated to align their beliefs with this loss of such freedoms. This process 
encourages individuals to perceive the change in circumstances in a more positive light, which in turn reduces cognitive dissonance that might have been associated with the loss of freedom (Festinger, 1957).

While cognitive dissonance and rationalization have been explored in numerous studies, the ways in which these processes are measured are difficult to apply retroactively and outside of a laboratory setting. Most studies of cognitive dissonance do not actually measure the experience of dissonance but instead infer whether the process occurred based on reported attitudes before and after the intervention, or attitudes after an experience that is known to be unappealing. For instance, Festinger and Carlsmith (1959) subjected participants to task known to be monotonous, then provided compensation to report the task was interesting to a subsequent participant. The researchers then gathered an evaluation of the participants' perspectives regarding the experiment. This study indicates that those who were paid one dollar to act as if the experiment was interesting rated the experiment as more interesting than those in the control, thus it was inferred that the process of cognitive dissonance occurred and the participants modified cognitions to align with their behavior (Festinger \& Carlsmith, 1959). In applied work, the study of cognitive dissonance has focused largely on perceived attitudes towards various political outcomes before and after their implementation (e.g., Beasley \& Joslyn, 2001), or on perceptions of political outcomes that might occur (e.g., Kay et al., 2002). Again, if cognitions towards the politician or political decision shift to be more in line with reality, inferences of cognitive dissonance and rationalization are made. Further, while a selfreport scale of cognitive consistency has been developed (Cialdini et al., 1995), the items 
in this measure focus on tendencies towards cognitive consistency as inherent to the person rather than dependent on context. Thus, this measure is inappropriate when trying to develop an understanding of the contextual factors that may play a role in the process of rationalization.

In the context of state policy for BIPs, the use of rationalization could occur if a program director learned of the standards which limit personal freedom to determine program practices and procedures, then changed their attitudes or beliefs to become aligned with the goals of the standards in order to reduce cognitive dissonance associated with making changes required by the policy. In order to explore the possibility that rationalization occurred among key program staff members of BIPs in Oregon, questions aimed at identifying cognitions prior to the introduction of standards, as well as after the introduction of standards were included in the interview. This use of questioning is similar to the ways in which rationalization is evaluated in applied work (e.g., Beasley \& Joslyn, 2001; Kay et al., 2002) with the caveat that there may be a significant time delay between the time when the program director learned of the standards and when they report their cognitions. While this is the case, it is interesting to understand the extent to which key program staff members recall experiences and shifts in attitudes. This information can be used to evaluate the extent to which negative attitudes shifted, for which rationalization may be one potential explanation. Specifically, the following questions were included:

- What was your initial response when you learned that standards were being implemented/were in place? 
- Providers might have both positive views of the standards, as well as concerns. What was your reaction?

- If at all, how have your thoughts about the standards changed over time? If your thoughts have changed, what has made them shift? If not, why do you think you still feel the same way?

- How do you feel about the standards now?

- Currently, what aspects of the standards seem to be consistent with your understanding of how to best work with abusive men?

- Currently, what aspects of the standards seem to be inconsistent with your understanding of how to best work with abusive men?

Based on the cognitive dissonance literature one important predication can be made. Specifically, it is expected that key program staff members who began with negative perceptions of the standards but have attempted implementation, will report that perceptions of the standards have become more favorable over time. If key program staff members report disagreement with the standards when they initially learned of them, cognitive dissonance theory predicts that in order to reduce dissonance key program staff members will change their perceptions of the standards to coincide with changes expected of the program. This may not be the case for key program staff members who have not implemented the standards, as they would not be in a state of cognitive dissonance (i.e., they have a negative view of standards and consistent with that view they do not implement components of the standards). Further, if key program staff members report experiencing high agreement with the standards initially, it is likely they 
did not experience cognitive dissonance and thus have not rationalized their cognitions towards standards.

Reactance. The second possibility suggested by the social psychological literature is that individuals may begin the process of reactance when faced with a situation that limits personal freedoms. The theory of reactance holds that individuals tend to have a strong desire to maintain the freedoms that are being restricted and respond negatively to those attempting to restrict such freedoms (Brehm, 1966). The notion of reactance is based on the premise that having the freedom to act and make decisions is a vital part of life (Brehm, 1966). Individuals are constantly utilizing this freedom to compare potential options and make decisions as to what, how, and when they will execute a given choice (Brehm, 1966). When this notion of freedom is challenged, individuals tend to react in ways that preserve the freedoms being threatened, thus making the threatened freedoms even more appealing than originally perceived (Brehm, 1966). This leads to a motivational desire to reinstate the freedoms that are being limited (Brehm, 1966). Not only does this process potentially lead to behaviors to try to maintain the freedoms being threatened, but it can also affect individuals' perceptions of the desired outcomes of the limiting decisions (Brehm \& Brehm, 1981). Thus, when an individual has freedoms taken away, he or she may display behavioral attempts to regain the freedom, as well as cognitive opposition to the ideals and premise of whatever policy or change was enacted that lead to the loss of freedom. For example, when freedoms are limited, the individual may feel as though they should be able to determine their own behavior, which may create feelings of anger and hostility that may be expressed verbally or nonverbally. 
Further, the individual will be inclined to participate in the freedom being limited (Brehm, 1966).

The degree to which reactance is experienced is dependent on whether or not freedom to make decisions was originally perceived, the proportion of freedoms being limited, as well as the relative importance of freedom (Brehm, 1966; Clee \& Wicklund, 1980). Therefore, when individuals did not originally perceive freedom, or when only a small proportion of their freedoms are limited, or if they do not view freedom as important, reactance may not occur.

In his original conceptualization of reactance, Brehm (1966) described the construct as a theoretical variable that cannot be measured (Erceg-Hurn \& Steed, 2011; Quick, 2012). While reactance was an immensely common theory in the field of social psychology (Donnell, Thomas \& Buboltz, 2001), rather than measuring the actual construct of reactance, researchers interested in this construct focused on the antecedents and outcomes of situations in which freedoms are limited (e.g., Mazis, 1975) (Dillard \& Shen, 2005). In 1983, a measure of reactance called the Questionnaire for the Measurement of Psychological Reactance, or QMPR, was developed (Merz, 1983). This questionnaire was originally developed in German and was translated into English and examined in English speaking countries (e.g., Australia and the United States) in order to establish measurement structure and stability (Donnell et al., 2001; Hong \& Ostini, 1989; Tucker \& Byers, 1987). These studies revealed inconsistencies in the factor structure and lead to the conclusion that the QMPR is psychometrically "unacceptable" and "unstable" and the possibility of creating an entirely new scale should be explored (Donnell et al., 
2001; Hong \& Ostini, 1989; Tucker \& Byers, 1987). A new psychological reactance measure was introduced by Hong (1992). This measure of reactance focused on reactance as a trait rather than a situation specific phenomenon (Hong, 1992). Items from this measure include: I consider advice from others to be an intrusion; and I become frustrated when I am unable to make free and independent decisions (Hong, 1992). This measure was later refined (Hong \& Faedda, 1996) and has come to be considered a measure of proneness to reactance. While this has been a valuable measure in the field, in 2005, two separate papers were published introducing two unique ways of measuring reactance that each take proneness to reactance into account but also extend to include aspects of context (Dillard \& Shen, 2005; Lindsey, 2005).

Dillard and Shen (2005) attempted to model the reactance process by integrating the examination of perceptions of a threat to freedoms, anger, cognitive responses, attitudes, behavioral intentions, and reactance proneness. These variables were included in order to determine whether the processes and reactions Brehm (1966) discussed, such as anger and desire to reinstate the threatened freedoms, as well as proneness to a reactive response (Hong, 1992) could be adequately measured. In order to test the model, two studies were conducted examining individual's responses to messages regarding either alcohol or flossing. In order to assess the model in its entirety, the two studies conducted by Dillard and Shen (2005) included both closed and open-ended survey assessments. Open-ended items were included to capture cognitions experienced after viewing the messages by asking respondents to write whatever came to mind immediately after viewing the message (Dillard \& Shen, 2005). The open-ended responses were coded in 
order to determine the extent to which positive, neutral, or negative cognitions towards the activity in question were experienced. In order to test the whole model, these responses were used in combination with closed-ended items aimed at assessing proneness to reactivity (Dillard \& Shen, 2005). This study indicates that the combination of all of these variables is superior to models that focus on just some aspects of the reactance process (Dillard \& Shen, 2005). Lindsey (2005) aimed to examine reactance by integrating the examination of proneness to psychological reactance, and the extent to which the individual responds negatively to the limit of freedom. This model was utilized to examine the relationship between reactance, guilt, and compliance. The introduction of two divergent methods for measuring reactance presented an opportunity to compare the two to determine which conceptualization and operationalization for measurement is most useful.

The comparison of the two measurement structures (Quick, 2012) revealed that the model created by Dillard and Shen (2005) appears to be superior. In the context of reliability, both measurement structures achieved high reliability as judged by Cronbach's alpha and Cohen's kappa, but the measure developed by Lindsey (2005) did not adequately differentiate threats to freedom and reactance (Quick, 2012). Further, when model fit indices were examined to determine validity of the two measures, the measure created by Dillard and Shen (2005) had better indices than the model introduced by Lindsey (2005). Thus, while both ways of measuring reactance may be viable, the measure developed by Dillard and Shen (2005) appears to be the better assessment of reactance. 
When examining the outcomes of the model in two contexts, drinking and flossing behaviors, it appears that threat to freedom and reactance proneness predict the latent variable of reactance which in turn predicts anger and negative cognitions. Additionally, the latent variable of reactance predicted attitude and behavioral intention in the alcohol sample, while reactance predicted attitude, which in turn predicted behavioral intention in the flossing sample. The good fit of these models indicates that the experience of rationalization is associated with a number of factors including threat to freedom, reactance proneness, attitudes, and behavioral intention.

The reactance model developed by Dillard and Shen (2005) includes items that aim to assess perceptions of the freedom threat, anger, and negative cognitions. Further, items from Hong's Psychological Reactance Scale (Hong, 1992; Hong \& Faedda, 1996) are included to capture proneness to reactance. Before discussing the content of the items it is important to note that Dillard and Shen (2005) examined reactance in the context of messages regarding drinking and flossing, therefore the language in the questions reflects that some type of message was presented to the participants. Examples of relevant items utilized by Dillard and Shen (2005) include: The message tried to make a decision for me; The message threatened my freedom to choose; Did you feel angry while viewing this message?; and, Did you feel annoyed while viewing this message? Dillard and Shen (2005) also examined attitudes towards the target behavior (e.g. drinking or flossing) through the use of Likert scale items that asked participants to identify positive or negative attitudes towards the behaviors. In order to understand cognitions regarding the messages, participants were asked to verbally report whatever they were thinking at the 
conclusion of the message. Behavioral intentions were also examined by asking participants to indicate on a scale of 1-100 the likelihood that they would engage in the behavior of interest in the next week.

Reactance as a consequence of introducing new policy has been studied in several contexts utilizing the approach introduced by Dillard and Shen (2005) including smoking prevention (Erceg-Hurn \& Steed, 2011) and voter mobilization (Mann, 2010). While reactance has been examined in the area of policy implementation, these studies tend to examine the experience of reactance based on changes to the individual (e.g., change in smoking policies) rather than changes to an organization that are enacted by an individual. Thus, the examination of the process of reactance due to policy that affects organizations and in turn those that own or direct the organizations would be an interesting avenue to pursue. If the process of reactance occurs in the context of BIPs, the choice to align beliefs and behaviors would go beyond affecting the individual BIP director and instead impact the program as a whole and the program participants. Hence, the implications for reactance may be increasingly extensive when experienced at the organizational level.

Given the support for the measurement model of reactance developed by Dillard and Shen (2005), which is based on Brehm's (1966) original conceptualization of the construct, it may be a valuable construct to include when examining key program staff members' responses to the introduction of state standards. In the context of standards for BIPs, the process of reactance may occur when key program staff members learn that they no longer have the same degree of freedom in developing the practices and 
characteristics of their program. If this freedom is viewed as important, the imposition of the standards could lead to resistance to the policy, a strong desire to maintain the removed freedoms, or negative perceptions towards the goals of the state standards. For example, the standards indicate certain criteria should be used to determine whether successful program completion has occurred. If key program staff members experience reactance to the standards and/or this component specifically, they may do what they can to maintain the completion requirements already utilized by the program rather than attempt to shift practices to those in line with the standards.

In order to gain a better sense of whether or not the process of reactance has occurred, items from the measure developed by Dillard and Shen (2005) have been modified for inclusion in the qualitative interview. These questions aimed to assess whether a threat to freedom was perceived and intent to comply with the standards. Additionally, the extent to which the program director is prone to reactance, anger, and negative cognitions towards the standards or the standards advisory committee, and attitudes towards the standards and the committee were probed with the interview questions. This information can be used to evaluate the extent to which negative attitudes were maintained, for which reactance may be one potential explanation. Specifically, the following questions were included:

- When did you become aware that standards were in place? How did you become aware? Did your initial awareness of the standards have any impact on how you made decisions about your program?

- Can you tell me more about how they impacted your ability to make decisions? 
- What was your initial response when you learned that standards were being implemented/were in place?

- Providers might have both positive views of the standards, as well as concerns. What was your reaction?

- Is your reaction to the standards similar or different to the response you have had to other policies that affect domestic violence?

- How do you feel about the standards now?

- Currently, to what degree do you believe your program practices and policies are in compliance with the standards? How do you gauge your level of compliance? Are you planning to change anything about your program practices that might impact compliance with the standards? Will this make your program more or less compliant?

- Currently, what aspects of the standards seem to be consistent with your understanding of how to best work with abusive men?

- Currently, what aspects of the standards seem to be inconsistent with your understanding of how to best work with abusive men?

In the context of the maintenance of negative attitudes towards the standards among BIP key program staff members' (i.e., a proxy for the possible experience of reaction), several predictions can be made based on the reactance literature described above. First, it is expected that those that describe the standards as a threat to their freedoms and describe typically responding negatively to policies like the standards will describe greater anger and negative attitudes, towards the standards. Next, it is predicted 
that those that provide deeper and more vivid experiences of maintaining negative attitudes will likely have more negative attitudes towards the standards and less intention to comply with the standards. Further, it will be interesting to investigate the relationship between attitudes, behavioral intention, and reported compliance in this context. If attitudes and behavioral intentions are predictive of reported compliance, it would be expected that those with more negative attitudes and less intention (i.e., possibly more reactance) will be key program staff members from programs with low rates of compliance.

Absoluteness. While the social psychological literature proposes these two quite different possible outcomes to the introduction of a new policy that restricts BIP practices (rationalization and reactance), Laurin and colleagues (2012) suggest a way to integrate these divergent frameworks by adding the concept of absoluteness. Absoluteness is the degree to which the enactment of the policy is certain, with some policies being more definitive than others (Laurin et al., 2012). While this phenomenon has not been extensively explored in the psychological literature, some recent work indicates that absoluteness may be an important determinant of these two reactions. It appears that absoluteness differentiates reactions to the introduction of policy that limits freedoms, with absolute polices being met with rationalization and non-absolute policies being met with reactance (Laurin et al., 2012).

This theoretical framework was first applied and supported in a study investigating reactions to changes in speed limit laws (Laurin et al., 2012). A sample of undergraduate students was assigned to three conditions: absolute policy change, non- 
absolute policy change, and control. In each condition the participants read an excerpt indicating that experts determined reduced speed limits would increase safety. Those in the absolute condition were told that a policy change based on this information would definitely occur. Those in the non-absolute condition were told that a policy change based on this information might occur if legislators vote in favor of the change. Those in the control condition were provided with no additional information beyond the expert report. After accounting for driving frequency, the absoluteness of the condition accounted for a significant amount of the variance in attitudes towards the policy change. More specifically, participants in the absolute condition viewed the policy change more favorably than those in the control condition and participants in the non-absolute condition viewed the policy change less favorably than those in the control condition (Laurin et al., 2012). This study provides preliminary support for the importance of absoluteness when attempting to understand reactions to policy.

This framework was next applied in a conceptual replication of the study described above which was conducted with a more representative United States sample rather than just college students (Laurin et al., 2012). The second study investigated reactions to the introduction of a policy that bans cell-phone use while driving. In order to examine absoluteness, four conditions were included: absolute policy change, nonabsolute but likely policy change, non-absolute but not likely policy change, and control. Additionally, the study utilized proposed policy changes for the United States and for India in order to gauge the impact of relevance to self in reactions. The findings from this study indicate that when the policy was proposed for the United States (i.e., relevant to 
oneself), there was a significant effect of absoluteness so that those in the absolute condition viewed cell phone use while driving more negatively than those in the nonabsolute conditions. This relationship did not hold when the policy was proposed for India (i.e., not relevant to oneself) (Laurin et al., 2012). These findings suggest that when a policy is relevant to oneself, the degree of absoluteness may inform the ways in which individuals react to the policy.

Thus, the work of Laurin and colleagues (2012) suggests that the more individuals feel the mandate of a given policy is inevitable, the more positively they will respond towards it and vice versa. This study is the first to investigate the role of absoluteness in differentiating responses to policy. Further exploratory work would likely prove beneficial in addressing possible factors to explain variability in responses to policy implementation, as well as the ways in which actual behaviors are impacted rather than just perceptions. Given these findings, an understanding of the processes of rationalization, reactance, and absoluteness may provide a useful lens for interpreting reactions to new policy in the context of BIPs

While the findings presented by Laurin and colleagues (2012) have not yet been replicated, they are useful in thinking about key program staff members' responses towards state standards. Because the standards in Oregon are not formally monitored or enforced and there is variation across counties in the extent to which monitoring occurs, there may be differing levels of absoluteness experienced by providers and differing perceptions of and reactions to the standards' policies. Gaining an understanding of the extent to which absoluteness is perceived and experienced by BIP key program staff 
members will aid in understanding key program staff members' responses to state standards. Further, this qualitative information could provide further explanation about these processes that may inform the framework proposed by Laurin and colleagues (2012). Acquiring detailed information about the ways in which key program staff members discuss the standards as absolute or non-absolute, in addition to their success in implementation and attitudes towards the standards, would add valuable information as to the validity of the theoretical link between the concepts of rationalization, reactance, and absoluteness.

While the experimental design employed by Laurin and colleagues (2012) was useful when examining hypothetical policy changes, the proposed study will be investigating reactions to actual policy. Therefore, the extent to which the policy is absolute cannot be manipulated. While this is the case, the current standards are not monitored or enforced in the same way across counties and it is plausible that because of the lack of enforcement there is variation in the extent to which the standards are viewed as absolute. Because absoluteness was manipulated in the context of the experiment rather than measured with a survey or interview assessment in the previous study (Laurin et al., 2012), a measure does not exist to inform the development of interview questions. In order to assess absoluteness, several questions that examine absoluteness from different perspectives were utilized. These questions will ask directly about absoluteness but will also gain information regarding features of the BIP standards context that may inform the extent to which absoluteness is experienced for each program director. The specific questions included: 
- What is your understanding of the consequences of not complying with the standards? Why? Has this view changed over time? What prompted those shifts?

- How much do you feel compliance with standards is expected or necessary? Who do you think expects compliance? How have your referral sources changed since the standards came about? How much do you think they value the standards? How has this impacted your program? Is anyone formally monitoring your compliance with standards now or have they in the past? Who?

Given the findings of Laurin and colleagues (2012) several predictions can be made regarding the role of absoluteness as it moderates the extent to which negative attitudes shift (i.e., possibly due to rationalization) or maintain (i.e., possibly due to reactance). First, it is expected that key program staff members who describe the standards as required and feel that relevant referral sources expect compliance will describe shifts in negative attitudes towards the standards (i.e., possibly due to rationalization). Conversely, key program staff members who describe the requirement of standards as ambiguous or unimportant and indicate that referral sources are not concerned with compliance will describe maintenance of negative attitudes towards the standards (i.e., possibly due to reactance).

Further predictions can be made when the entire framework suggested by Laurin and colleagues (2012) is considered. While predictions are made based on the work of Laurin and colleagues (2012) it is important to note that rationalization and reactance could not be measured perfectly. Because of this, negative attitude change and maintenance will serve as a proxy for the experiences of rationalization and reactance as 
these social psychological experiences may be one potential explanation for the change and maintenance of negative attitudes. First, it is expected that key program staff members who describe the standards as absolute will describe experiences that more typically reflect rationalization, as operationalized in the current study as a shift in negative attitudes towards the standards. Second, it is expected that key program staff members who describe standards as non-absolute will describe experiences that more typically reflect reactance, as operationalized in the current study as the maintenance of negative attitudes towards the standards.

Legitimacy. While the framework proposed by Laurin and colleagues (2012) is useful to differentiate responses to the introduction of policy, the construct of absoluteness may be more informative when examined along with the construct of legitimacy. Legitimacy refers to the extent to which those in power, whether the power holder is an individual or organization, are believed to make fair and appropriate decisions (Tyler, 2006). The conceptualization of legitimacy stems from work examining authority, social systems, and ways in which power is achieved (French \& Raven, 1959; Weber, 1968). Legitimacy is one way to exert power and influence others (French \& Raven, 1959). While other methods of inducing compliance, such as coercive power and reward power (French \& Raven, 1959) have been identified, the use of these tactics tends to be both costly and limited in effectiveness (Tyler, 2006). Some of the limitations to the use of coercive power and reward power stem from the costs associated with ensuring rules are being followed, carrying out punishments for those not in adherence, and providing incentives for those successfully following the rules or guidelines set forth. 
Given the costs associated with coercive power and reward power, the use of legitimacy to encourage adherence to rules and policies is an attractive alternative (Tyler, 2006).

When those in power are perceived as legitimate they experience greater latitude for making decisions and rules to which others will adhere (Tyler, 2006). The link between legitimacy and adherence to guidelines or polices has been demonstrated in numerous settings including decisions of police officers and judges (Tyler \& Huo, 2002), recommendations of doctors (Stevenson, Britten, Barry, Bradley \& Barber, 2002), and educational policy decisions (Wallner, 2008). Thus, when attempting to understand implementation of a policy that does not have formal sanctions for non-adherence, such as Oregon state standards for BIPs, perceptions of legitimacy may aid in explaining variance in compliance.

When examining perceptions of legitimacy in connection to the introduction of a new policy, it is necessary to identify qualities of the authoritative body or policy that may impact legitimacy. In general, perceptions of legitimacy are influenced by three key factors: procedural justice, social norms, and logic of the policy. Procedural justice originates in the work of Thibaut and Walker (1975) and refers to the extent to which individuals feel that those making decisions are doing so fairly (Barrett-Howard \& Tyler, 1986; Tyler, 2006). When those in authority positions are perceived as making decisions fairly, they tend to be viewed as more legitimate (Tyler, 2006). In an extensive review of the literature examining the construct of legitimacy Tyler (2006) noted that the relationship between perceptions of fairness and procedural justice and legitimacy has been demonstrated in numerous domains including legal, political, and workplace 
settings. When applied to the context of BIP key program staff members' implementation of standards, it may be important to develop an understanding of perceptions of procedural justice and fairness in order to fully assess perceptions of legitimacy. The relationship between procedural justice and legitimacy drives the prediction that those who perceive the way in which the standards were administered, as well as the body responsible for the standards, as fair will perceive the standards as more legitimate. Conversely, those that view the introduction of standards or the body responsible for the standards as unfair will perceive the standards as less legitimate.

While procedural justice is one determinant of legitimacy, social norms and policy logic are two additional determinants of legitimacy that must be considered. Social norms in this context refer to the extent to which others impacted by the authority figure or policy view the figure or policy in a positive or negative light. Specifically, the more that people believe that others support the authority figure or policy, the more legitimate that authority figure or policy will appear (Tyler, 2006; Zelditch, 2001; Zelditch \& Walker, 2000). It is important to point out that norms are transmitted through interactions with others (Cialdini \& Trost, 1998). The transfer of norms requires some type of interaction but the way in which norms are transferred can be deliberate, passive, or through inference (Cialdini \& Trost, 1998). Further, norms can be thought of as descriptive, injunctive, or subjective (Cialdini \& Trost, 1998). Descriptive norms specify what is usually done, injunctive norms specify what is believed to be appropriate in society, and subjective norms specify what relevant others believe to be appropriate 
(Cialdini \& Goldstein, 2004; Cialdini \& Trost, 1998). Each of these types of norms may be important depending on the salience of the norm and context (Cialdini \& Trost, 1998). When applied to state standards for BIPs, in order to understand legitimacy it will be necessary to assess key program staff members' perceptions of the social norms regarding the state standards. First, information regarding the nature of contact that key program staff members have with others involved with the standards should be assessed. Those key staff members who have very little contact with or exposure to others in the batterer intervention field may have less accurate perceptions of what is normative as compared to key program staff members who are highly active in the field. Next, as Cialdini and Trost (1998) note, norms are transmitted through interaction. Thus it will be valuable to assess the ways in which norms regarding standards are transmitted in the BIP community. For instance, if the Standards Advisory Committee would like to provide education or elicit discussion about the standards, it will be valuable to determine whether norms surrounding the standards are transmitted during formal trainings and meetings, or if norms regarding standards are transmitted in less formal settings. This information may provide insight into the settings and contexts where providers naturally discuss standards that may be a potential setting for education and discussion. For instance, if the providers indicate that most discussion relevant to the standards occurs in formal county meetings for BIP directors and facilitators, this could indicate that education and discussion about standards could be introduced within these meetings. Conversely, if providers indicate that most discussion of the standards occurs in informal contexts, such as one-on-one conversations among providers, this may indicate that 
education and discussion about standards should be approached more individually. Finally, the content and degree of the norms can be assessed. Based on the conceptual link between social norms and legitimacy it is expected that key program staff members who describe positive social norms towards the standards will view the standards as more legitimate. These norms may be described as descriptive (e.g., I see other program directors implementing the standards); injunctive (e.g., I think most program directors like the standards); or subjective (e.g., the program directors I am close to all think the standards are a good thing). Conversely, those that describe negative social norms towards the standards will view the standards as less legitimate.

The final element of legitimacy that must be discussed is the logic or rationale of the policy. When policy creation is based on scientific evidence or according to best practices in the field, it should be viewed as more legitimate than when the creation process does not incorporate evidence or best practice (Stryker, 1994; Wallner, 2008). In order to create policies that have a strong logic, policymakers can incorporate empirical evidence to demonstrate the rationale for the given policy and its likely impact. Alternatively, policymakers can incorporate experiences of key stakeholders to develop policy that accounts for stakeholder experiences. When stakeholders are included in the policy development process it may take longer to create policy, but the inclusion of stakeholders is associated with increased perceptions of legitimacy (Wallner, 2008). This aspect of legitimacy will be interesting to assess in the context of BIPs. As noted previously, critics of standards have voiced concerns regarding the extent to which standards are supported by empirical evidence (Austin \& Dankwort, 1999; Gelles, 
2001;Holtzworth-Munroe, 2001). If providers in Oregon share this concern, they may view the standards as less legitimate. Conversely, because standards were created through a collaborative committee that includes key stakeholders, it is possible that they will be perceived as more legitimate. In order to assess perceptions regarding the logic of the standards, views concerning the content of standards must be obtained. Specifically, information from key program staff members regarding the extent to which they endorse the content of the standards and why will be vital to understanding how content logic impacts legitimacy. It is expected that those that endorse the content of the standards, whether it be due to perceived empirical validity or the use of a collaborative committee, will perceived the standards as more legitimate than those that do not endorse the content of the standards.

Legitimacy and its determinants can be assessed in the context of BIP standards in order to better understand the extent to which legitimacy varies and how perceptions of legitimacy relate to policy implementation. Studies of legitimacy in policy implementation have included measures that are highly dependent on context. For instance, in order to study the legitimacy of U.S. national policy, items assessing agreement with statements relevant to national policy decisions such as war and the environment have been utilized (Fraser, 1974). To study the legitimacy of Supreme Court policy decisions, questions were constructed assessing the fairness and agreement with various Supreme Court decisions (Mondak, 1994). When the legitimacy of educational policy was assessed, questions regarding stakeholder involvement, procedural justice, and policy content specific to educational policy in two specific geographic locations were 
asked (Wallner, 2008). These examples point to the need for contextually specific questions that aim to gather information regarding components of legitimacy, as well as legitimacy more generally. In line with these examples, interview questions specific to the context of BIP standards were developed. These questions include:

- Can you describe how you feel about the creation of state standards? What about the people involved in this process? What are your thoughts about creating state policy to influence program practices? Do you believe that the individuals who participate on the Standards Advisory Committee represent most providers? Why do you think this? (Procedural Justice/Legitimacy)

- Can you describe the extent to which you are involved in the batterer intervention community in Oregon? Do you discuss the standards with other providers? What do you discuss? Can you tell me about how those in the field see the standards? Do you agree with the consensus in the field? Why or why not? (Social Norms)

- Currently, what aspects of the standards seem to be consistent with your understanding of how to best work with abusive men? Currently, what aspects of the standards seem to be inconsistent with your understanding of how to best work with abusive men? Why do you think this is the case? Probe: Do you think they have been created from evidence-based practices? Do you think they have been created from best practices in the field? Are your feelings about the standards related to how they were developed? Is there anything you would change about the standards? (Policy Logic) 
These questions aim to not only gather an overall sense of the extent to which legitimacy of the standards is perceived, but also to develop an understanding of the three determinants of legitimacy. Based on the legitimacy literature, several predications can be made. First, those with stronger perceptions of procedural justice, stronger positive social norms, and stronger perceptions of policy logic will express stronger experiences of legitimacy. Further, it can be predicted that those with stronger experiences of legitimacy will be those with higher compliance. Conversely, lower perceptions of procedural justice, more negative views of social norms, and lower perceptions of policy logic will be expressed by those who believe the standards have less legitimacy. Further, lower legitimacy will correspond with lower compliance.

Social action research. Social action research has its roots in the 1940s in the work of Kurt Lewin (Dash, 1999; Smith \& Doyle, 2007). Lewin (1946) recognized the importance of academic research but also asserted, "Research that produces nothing but books will not suffice" (p. 35). Lewin (1946) stressed the importance of integrating knowledge across fields and incorporating the nuances of context into research studies rather than exclusive study of basic processes and universal laws. Social action research not only takes these goals into account, but goes beyond diagnosing problems by attempting to identify potential solutions (Lewin, 1946). The ideals presented in a social action research framework contribute significantly to this study of policy implementation.

In the context of state standards for BIPs in Oregon, the ideals of social action research can be applied in several ways. First, the unique history of standards development in the state of Oregon must be accounted for when investigating how 
standards have been implemented. A clear understanding of who created the standards and the method by which they were imposed on key program staff members will contribute to a comprehensive analysis of why there are varying degrees of compliance with the standards. Second, the use of information from multiple disciplines is vital to understanding how key program staff members have responded to the standards. The fields of social psychology, interdisciplinary violence studies, and policy implementation each contribute unique knowledge and context. Such knowledge is necessary to determine how the standards have or have not been implemented across Oregon. Third, because the process of social policy is contingent on time and place, the goal of social action research to impact the community may be especially valuable. The Standards Advisory Committee is able to continually review and suggest modifications to the Oregon state standards, though historically changes have been made infrequently. Information from key program staff members regarding their attitudes towards the standards and their experiences with implementation may have a profound and direct impact on the content of the standards and/or the way in which programs are supported in implementation. Finally, because there is no formal statewide organization that gathers information about BIPs across Oregon, this research study investigating policy implementation directly serves the BIP, criminal justice, and IPV victim communities by producing a statewide directory of BIP programs that was distributed to BIPs, probation departments, victim advocates, and was posted on the Oregon Department of Justice website. Additionally, the directory alone may encourage implementation because it serves as a resource to connect BIPs to one another and to other relevant agencies, such 
as probation and victim advocacy. Further, the procedures of this study will provide contact with BIP key program staff members and allow this resource to be updated so that the referral information available in Oregon is current and practically useful. Each of these factors positions a study of the implementation of standards to answer Lewin's (1946) call for social action research and contribute to the larger community in practical and discrete ways.

\section{Previous Research on Compliance with Oregon BIP Standards}

Previous studies have surveyed national samples in order to understand the prevalence of various state standards or the policies and practices of programs (Maiuro \& Eberle, 2008; Price \& Rosenbaum, 2009). Only one study has examined how BIP practices and characteristics differ before and after the implementation of state standards (Boal, 2010). My advisor and I, in collaboration with numerous community partners, initiated the first study of its kind to identify all programs in the state of Oregon, gain insight into whether programs comply with the standards, and circulate the results of the study statewide to impact further policy development and implementation. BIP directors from all BIPs in the state of Oregon were surveyed in 2001, 2004, and 2008 and asked to report on the practices and policies of their program. The data collected through these surveys was utilized first to develop a statewide directory of BIPs. The purpose of the directory is to provide information to judges, probation officers, clinical psychologists, victim advocates, and others who may be interested in BIPs, improve referrals, and increase knowledge about BIP practices. 
After the directory was completed, these data were evaluated extensively in my master's thesis project to accomplish two goals relating to understanding compliance with state standards. In this study, compliance was gauged based on several important practices and policies assessed at each time-point; although these represent only a small fraction of all requirements included in the state standards. The specific practices and policies include community collaboration, requirements for program completion, program length, mixed-gender co-facilitation, and education of facilitators. The first goal was to determine the extent to which compliance with the state standards changed over time in relation to the implementation of standards in 2006. The second goal was to determine the degree to which programs in 2008 were complying with the standards. This study revealed that compliance with the selected practices and policies increased descriptively over time and that programs in 2008 were complying with $72 \%$ of the practices and policies analyzed (Boal, 2010).

While the previous study shed light on the degree to which programs were in compliance with some important components included in the standards, it did not give a complete picture of the degree to which standards had been implemented. Specifically, the previous study examined compliance exclusively in the context of community collaboration, completion requirements, program length, mixed gender co-facilitation, and education of facilitators. This study of compliance provided an initial examination of how well programs are adhering to the standards; however, a comprehensive investigation of program compliance requires examination of additional components included in the standards. For example, compliance with important components such as 
the content of written policies and procedures, victim contact procedures, the content of program curriculum, and causes of battering endorsed by the program were not examined in the previous study. To fully understand compliance, all aspects of program functioning that are explicitly addressed in the standards should be examined.

Further, the previous study did not sufficiently answer questions about promoting policy implementation in the context of BIP standards. For instance, the analysis did not comprehensively identify programs' challenges with implementation of practices required by the standards, or the degree to which programs were in agreement with the various components of the standards. Information relevant to key program staff members' attitudes towards the standards as a whole, in addition to individual components, experiences with implementation, barriers and facilitators to implementation, and perceived importance of the standards will contribute to an understanding of how policy implementation has occurred, what can be done to encourage implementation in the future, and possible reasons that the standards should not be implemented.

\section{Current Study}

Overview. Given the lack of knowledge regarding reactions and responses to the introduction of a new policy and the policy implementation process for BIPs, I evaluated the success and process of the implementation of BIP standards in Oregon. In order to accomplish this, I first assessed the extent to which BIPs in Oregon are currently in compliance with state standards. To better understand programs' responses to state standards, I then interviewed providers to identify of how programs adapt to standards, attitudes towards the standards, and what resources may equip them to achieve a higher 
degree of compliance. I examined program directors' or key staff members' reactions and responses to implementation utilizing several social psychological lenses. Program directors were first identified as the desired participant from each program due to the authoritative role in setting policies and procedures that program directors play within their organizations. In some instances program representatives were nominated by program directors due to their central role in the batterer intervention component of the larger program. Program directors and key representatives were selected due to the crucial role they play in ensuring the components of standards are or are not implemented. These individuals are knowledgeable about the extent to which implementation has occurred and the process of implementation within their program. Additionally, program directors and key representatives are best poised to take action in the future to increase or decrease compliance and are thus the most influential individuals for program change as it relates to standards. The social psychological constructs of perceived control, actual control, attitude change (including the potential explanations for attitude change of rationalization and reactance), absoluteness, and legitimacy were used to interpret the data (see Figure 2). The use of these analytic lenses provided a conceptual background to make sense of program representatives' experiences as they relate to policy implementation. I achieved these goals through use of a social action research framework, including the creation of a directory of BIPs in Oregon and the dissemination of findings back to policymakers, in order to ensure a socially relevant and useful study. In sum, I aimed to generate information that will be useful for both knowledge and practice. I attempted to provide novel insight into the processes that are employed 
when a new policy is enacted in the BIP realm. I also intended to contribute rich and detailed information that may be useful to build upon current theoretical thinking regarding perceived control, actual control, negative attitude change (including the potential explanation of rationalization) and maintenance (including the potential explanation of reactance), absoluteness, and legitimacy in this particular context. Beyond the theoretical benefits, the study offered data that can be directly applied to support programs in achieving better implementation or in effecting policy content. In order to accomplish these goals, I sought to answer four research questions and using two distinct phases. Consistent with the action research focus of the project, the content of the research questions were influenced by the needs and interests of the Oregon Attorney General's BIP Standards Advisory Committee. The committee was particularly interested in learning about the barriers and facilitators to implementation. These needs and interests were incorporated into the questions asked in the study. The content of the research questions and the details of each phase are described below.

Research questions. The previous sections have examined the social problem of IPV, one response to the problem of IPV in the form of BIPs, the use of state standards intended to influence BIP practices and characteristics, and the importance of understanding the policy implementation process. In order to fully appreciate the impact of state standards on BIPs, relevant social psychological processes that may impact implementation were assessed. The examination of these areas provides the necessary background and context for the development of four primary research questions, as well as relevant sub-questions and hypotheses, aimed at understanding BIP representatives' 
responses to state standards. The justification for each research question and hypothesis is described below.

The high prevalence of IPV in the United States (Catalano, 2007; Coker et al., 2000; Tjaden \& Thoennes, 1998; Tjaden \& Thoennes, 2000; Thompson et al., 2006) coupled with the common use of BIPs as a mandated intervention for perpetrators of IPV (Bennett \& Williams, 2001; Gondolf, 1997) points to the importance of understanding the current functioning of BIPs. While previous studies have examined the practices of BIPs (e.g., Price \& Rosenbaum, 2009) the use of state standards to prescribe and proscribe BIP functioning across the United States (Maiuro \& Eberle, 2008) indicates that not only do practices need to be understood, but they also must be examined within the context of standards. Currently, only one study (Boal, 2010) has examined these practices in the context of state standards. While this previous study provided initial information regarding practices as they relate to state standards, only a few components of standards were evaluated. A more thorough investigation of the ways in which BIPs function in light of standards is needed. Thus, the purpose of this study was to develop a comprehensive understanding of BIP practices as they correspond to state standards, program representatives' responses to the standards and implementation strategies, as well as the extent to which program representatives from programs that comply with the standards to a high degree or low degree differ in their responses and implementation strategies. Hence, the first research question is addressed using survey data on a comprehensive range of current practices and policies of BIPs as they relate to state standards. These data on BIP characteristics enable analysis of the extent to which 
programs are in compliance with the state standards. Compliance is operationalized as the average number of compliance characteristics achieved across all possible compliance characteristics assessed. This first research question is descriptive in nature and therefore no predictions regarding which practices or policies are most prevalent were made.

Research question one (RQ1). What are the current practices and policies of BIPs in Oregon?

In order to determine the extent to which compliance with standards has occurred, as well as the rationale for variations in compliance, further information regarding the ways in which program representatives have responded to the introduction of standards should be assessed. The social psychological literature may be especially useful in understanding how individuals respond to the introduction of a new policy (Bauer, 1965; Esses \& Dovidio, 2011; Fischhoff, 1990) and thus can be utilized as an analytic lens to better understand how the introduction of standards was, and contributes to be, experienced by program representatives. Specifically, several constructs from the social psychological literature may be useful in understanding the extent to which compliance has been achieved and why variation in compliance may occur. These constructs include perceived control, actual control, negative attitude change (including the potential explanation of rationalization) and maintenance (including the potential explanation of reactance), absoluteness, and legitimacy. Given this analytic lens, I proposed the second research question to address the various responses and reactions to the introduction of the standards. In addition to the overarching research question, I proposed seven subquestions to examine specific social psychological processes that may impact responses 
to the standards. Due to the lack of previous research regarding the ways in which social psychological processes impact responses to BIP standards, the majority of sub-questions do not include hypotheses as hypotheses were only included when the literature supports their inclusion.

Research question two (RQ2). How do program representatives in Oregon react and respond to state standards?

Research suggests that the extent to which individuals have actual control over their behaviors or the policies that will subsequently impact their behavior may influence their thoughts and beliefs about the behavior or policy (Ajzen \& Madden, 1986; Becker et al., 2002; Kieffer, 1984; Schulz et al., 1994; Wortman, 1975). Actual control is necessary to consider because having actual control may indicate that the individual has greater access to resources (e.g., collaborative partners, trainings, etc.) relevant to the outcome of interest (e.g., compliance with state standards) than those that do not have actual control (Ajzen\& Madden, 1986). Thus, in order to describe responses to the state standards it is important to determine the extent to which the program representatives were involved in the creation or refinement of the standards.

$R Q 2 a$. To what extent do program representatives report having actual control over the content and development of the standards?

In addition to actual control, the social psychological literature also points to the importance of perceived control when responding to the introduction of a new policy. The extent to which individuals believe that they can meaningfully contribute to and impact a policy that will affect them may influence the extent to which they are in 
agreement and alignment with the policy (Itzhaky \& York, 2000; Ohmer, 2008; Paulhus, 1983; Zimmerman \& Zahniser, 1991; Zimmerman, Ramirez-Valles \& Maton, 1999). Therefore, it is vital to determine the extent to which program representatives perceive having control over the development, content, and scope of the standards.

$R Q 2 b$. Do program representatives perceive having control over the content and development of the standards?

The experiences of actual control and perceived control do not exist in isolation. Studies examining the effects of actual control on perceived control in the sociopolitical context have found that experiences of actual control are predictive of perceived control (Becker et al., 2002; Kieffer, 1984; Schulz et al., 1994). Therefore, it is hypothesized that those who describe higher actual control in the form of participation in the creation or refinement of the standards would also describe higher perceived control in the form of beliefs that they would be able to impact the content and scope of the standards if they desired to do so.

Hypothesis $2 a(H 2 a)$. Program representatives who primarily report having actual control over the standards will describe higher perceived control as compared to those who primarily report not having actual control over the standards.

The social psychological literature suggests that in response to the introduction of a policy that limits freedoms, one potential response is that of rationalization. Rationalization is the process by which individuals undergo cognitive processes to modify their views of a policy that limits freedoms in order to perceive that policy in a 
more positive light (Festinger, 1957; Nail \& Boniecki, 2011; Thibodeau \& Aronson, 1992). Because an assessment of program representatives' attitudes when the standards were first introduced was not conducted, the study utilizes retrospective recollections of initial responses to the standards in order to attempt to gauge the experience of rationalization. While there are limitations to this approach, research demonstrates that rationalization is associated with positive views of the behavior or policy in question (Beasley \& Joslyn, 2001; Granberg \& Brent, 1983; Kay et al., 2002). Thus, the current study will examine retrospective accounts of the change in negative attitudes towards the standards, with the understanding that one potential explanation for this change is the process of rationalization.

$R Q 2 c$. Do program representatives describe responses to the standards consistent with the phenomenon of rationalization?

While the shifting of attitudes to become more positive (i.e., possibly the process of rationalization) is one possible outcome to the introduction of a policy that limits freedoms, the maintenance of negative attitudes (i.e., possibly the process of reactance) is another potential outcome. Research demonstrates that reactance is associated with negative perceptions and responses to a policy that limits freedoms (Dillard \& Shen, 2005; Erceg-Hurn \& Steed, 2011; Mann, 2010). Despite limitations in measurement of reactance in real-world, retrospective scenarios, acquiring some knowledge about this phenomenon may shed light onto whether participants tend to maintain negative perceptions. Given the relationship between reactance and negative perceptions of a given policy, determining the extent to which reactance may have occurred will be useful 
in developing an understanding of program representatives' reactions and responses to the standards and the way in which these reactions and responses were generated. Thus, the current study will examine the maintenance of negative attitudes towards the standards, with the understanding that one potential explanation for this attitude consistency is the experience of reactance.

$R Q 2 d$. Do program representatives describe responses to the standards consistent with the phenomenon of reactance?

Laurin and colleagues (2012) suggest that absoluteness of a policy that limits freedoms aids in differentiating reactions of rationalization and reactance. Absolute policies are met with rationalization and non-absolute policies are met with reactance (Laurin et al., 2012). While rationalization and reactance could not be measured directly, the retrospective accounting of change or maintenance in initial negative attitudes towards the standards served as a proxy for these constructs. Thus, I attempt to further evaluate this framework with one sub-question and two hypotheses that aim to at determine whether those who retrospectively report changed their initial negative attitudes towards the standards (i.e., a proxy for rationalization) view the standards as primarily absolute and those who retrospectively report maintained their initial negative attitudes towards the standards (i.e., a proxy for reactance) view the standards as primarily non-absolute.

$R Q 2 e$. Do program representatives view the standards as an absolute policy? 
Hypothesis $2 b(\mathrm{H} 2 b)$. Program representatives who respond to the standards with rationalization will view the standards as more absolute than program representatives who respond to the standards with reactance.

Finally, I apply the social psychological construct of legitimacy to program representatives' responses to the standards. Legitimacy has been examined in relation to three key factors: procedural justice, social norms, and policy logic (Tyler, 2006). Individuals' views regarding these three aspects combine to determine the extent to which a policy or authoritative body is viewed as legitimate. Research indicates that the extent to which an authoritative body and a policy are viewed as legitimate impacts whether or not individuals view the policy favorably (Stevenson et al., 2002; Tyler, 2006; Tyler \& Huo, 2002; Wallner, 2008). Given the relationship between legitimacy and favorable perceptions of a policy or administrative body, it is important to detect whether or not program representatives in Oregon view the standards and the Standards Advisory Committee as legitimate.

$R Q 2 f$. Do program representatives perceive the standards and the process by which the standards were created as legitimate?

While ascertaining how current program representatives have responded to the standards is important, perhaps the most extreme result of the introduction of state standards is the closing of a program due to the requirements of the standards. No research has been conducted to investigate the role that standards may play in program closures. In order to identify the extent to which state standards have impacted program 
closures, Oregon BIPs that have stopped providing BIP services since the last assessment of Oregon BIPs was completed in 2008 were contacted about whether or not the standards impacted their ability to provide BIP services. Because there is no research that has been done to inform this question and the possible reasons for program closure are potentially vast, no hypotheses are proposed. Instead, the inquiry focuses on identifying the role standards played, if any, in program closures across the state.

$R Q 2 g$. How do state standards impact BIP closures across the state of Oregon?

The third research question asks how programs have implement standards and seeks to identify and explain this process through the examination of five sub-questions. While there is literature regarding policy implementation generally (e.g., Barrett, 2004; Berman, 1974; Elmore, 1979; Matland, 1995; McLaughlin, 1987; O’Toole, 1986; 2004), hypotheses are not proposed for this research question due to the lack of relevant knowledge and theory regarding policy implementation in the context of BIPs. The goal of this research question was explain the process of implementation in order to provide practical knowledge that can be utilized by policymakers.

Research question three (RQ3). How do BIPs in Oregon implement State standards?

In order to assess the ways in which standards have been implemented, it is first necessary to gain an understanding of the ways that program representatives have attempted to implement the standards. This information will provide a description of the tactics employed by BIPs in their attempts to meet the standards. Knowledge of 
implementation strategies will allow insight into the extent to which program representatives report similar or differing processes of implementation. Further, this will provide insight as to the processes of top-down and bottom-up implementation (Barrett, 2004; Berman, 1974; Elmore, 1979; Matland, 1995; O’Toole, 1986; 2004) and how these processes were incorporated throughout implementation. Further, because policy implementation is not a linear process (McLaughlin, 1987) this question will provide information regarding the possibly nonlinear implementation process.

$R Q 3 a$. What specific strategies have program representatives used to implement the standards?

In addition to gaining information regarding the strategies utilized to implement the standards, this study originally sought to identify program policies and characteristics have and have not changed prior to and following the adoption of standards. While it was believed that this information would provide insight into the aspects of program functioning that were already aligned with standards, aspects that were misaligned but were successfully changed, and aspects that were misaligned and continue to be misaligned the way in which the interview guide was structured focused responses on which components of the standards had and had not changed since the introduction of the standards. Because of this, it was not possible to determine which components of the standards had not changed due to the policy or practice already being in place versus lack of change due to the program's inability to enact the component. Thus, the following research question was adapted to better suit the data at hand and identify the program policies and characteristics that program representatives describe as more or less difficult 
to successfully implement. Identifying policies and characteristics that are relatively easy and relatively difficult to implement will highlight the components of the standards with which programs did and did not experience challenges in implementation.

$R Q 3 b$. Which program policies and characteristics are described as relatively easy and relatively difficult to implement by program representatives?

In order to provide practically useful information regarding what helps programs achieve compliance, identifying the perceived enablers to compliance is important. This information may be useful in determining what helps programs successfully implement standards. The characteristics commonly examined in the policy implementation literature described by O'Toole $(1986 ; 2000 ; 2004)$ will be initially utilized to aid in differentiating types of factors that aid implementation. These include: policy characteristics; resources; implementation structure; the number of individuals responsible for implementation; attitudes and perceptions of implementing personnel towards the policy and its feasibility; alignment of clientele with the content and purpose of the policy; and timing of the policy relative to the political and economic climate (O’Toole, 1986).

$R Q 3 c$. What factors enable BIPs' compliance with state standards?

Conversely, while enablers to compliance are important to note, it is also valuable to determine what has made compliance with the standards more difficult. Again, the characteristics commonly examined in the policy implementation literature described by O’Toole $(1986 ; 2000 ; 2004)$ will be initially utilized to aid in differentiating types of 
barriers. These include: policy characteristics; resources; implementation structure; the number of individuals responsible for implementation; attitudes and perceptions of implementing personnel towards the policy and its feasibility; alignment of clientele with the content and purpose of the policy; and timing of the policy relative to the political and economic climate (O’Toole, 1986).

$R Q 3 d$. What factors are barriers to BIPs' compliance with state standards? The final sub-question pertaining to the process of implementation is included to provide explicit feedback to policymakers regarding the needs of program representatives. In this question I ask about resources and sources of support that program representatives believe impact their ability to implement the standards. The information gained through this sub-question will be directly applicable to informing the Standards Advisory Committee about what would enable or encourage implementation.

$R Q 3 e$. What needs do program representatives identify in order to successfully implement the standards?

Data addressing $R Q 1, R Q 2$, and $R Q 3$ provide extensive descriptive information regarding current BIP practices and policies, responses and reactions to the state standards, and the process of implementation. The final research question is based in the relevant social psychological constructs examined in $R Q 2$ and asks what factors differentiate compliance with the standards. As previously mentioned, compliance is operationalized as the average number of components of the state standards for which each program reports adherence. Programs with the highest and lowest average compliance were identified to determine whether various social psychological processes 
differentiate high and low compliance programs. In order to achieve this, I proposed five hypotheses.

Research question four (RQ4). Do the responses and reactions to standards differ for programs with different levels of compliance?

As has been described, actual control is associated with greater resources to achieve the desired outcome (Ajzen \& Madden, 1986; Durlak \& DuPre, 2008; Wortman, 1975). Thus, I expect that program representatives from high compliance programs will report experiences of actual control at a greater frequency and depth as compared to program representatives from low compliance programs.

Hypothesis $4 a(\mathrm{H} 4 \mathrm{a})$. High compliance program representatives will describe relatively more experiences of actual control as compared to low compliance program representatives.

Perceived control has been shown to be associated with positive outcomes in the sociopolitical realm (Holden et al, 2004; Itzhaky \& York, 2000; Ohmer, 2008). Thus, I expect that program representatives from high compliance programs will report perceived control at a greater frequency and depth as compared to program representatives from low compliance programs.

Hypothesis $4 b(\mathrm{H} 4 b)$. High compliance program representatives will describe relatively more experiences of perceived control as compared to low compliance program representatives.

Studies of rationalization in the sociopolitical context have suggested that when individuals engage in the process of rationalization they view the policy change more 
favorably and align their beliefs and behaviors with the new policy (Beasley \& Joslyn, 2001; Granberg \& Brent, 1983; Kay et al., 2002). Rationalization could not be perfectly assessed in the current study. Thus, the current study will examine the change in negative attitudes towards the standards as it relates to compliance with the understanding that one potential explanation for this attitude change is the process of rationalization. Hence, I expect that program representatives from high compliance programs will report changing negative attitudes towards the standards to be primarily positive (i.e., a proxy for rationalization) at a greater frequency and depth as compared to program representatives from low compliance programs.

Hypothesis $4 c(\mathrm{H} 4 \mathrm{c})$. High compliance program representatives will describe relatively more reactions consistent with rationalization as compared to low compliance program representatives.

Theory and studies relevant to reactance suggest that when individuals engage in the process of reactance they view the policy change less favorably and try to resist changes associated with the given policy (Brehm, 1966; Dillard \& Shen, 2005; ErcegHurn \& Steed, 2011; Mann, 2010). Reactance could not be perfectly assessed in the current study. Thus, the current study will examine the maintenance of negative attitudes towards the standards as it relates to compliance with the understanding that one potential explanation for this attitude consistency is the experience of reactance. Given the findings in the reactance literature, I expect that program representatives from low compliance programs will report maintenance of negative attitudes towards the standards (i.e., a 
proxy for reactance) at a greater frequency and depth as compared to program representatives from high compliance programs.

Hypothesis $4 d(H 4 d)$. Low compliance program representatives will describe relatively more reactions consistent with reactance as compared to high compliance program representatives.

The legitimacy literature suggests that perceptions of legitimacy are associated with adherence to guidelines and polices in diverse settings (Tyler \& Huo, 2002; Stevenson et al., 2002; Wallner, 2008). Therefore, I expect that program representatives from high compliance programs will report legitimacy at a higher frequency and greater depth as compared to program representatives from low compliance programs.

Hypothesis $4 e(\mathrm{H} 4 \mathrm{e})$. High compliance program representatives will describe relatively more perceptions of the standards and process of standards creation consistent with legitimacy as compared to low compliance program representatives. 


\section{Chapter 2: Method}

In order to address the four research questions and eight hypotheses proposed in the current study, quantitative and qualitative data collection and analysis methods were utilized. This study employed a sequential mixed methods design with a preliminary quantitative component and principal qualitative component (Morgan, 1998). This type of design was selected due to its strengths in answering each of the research questions and the complementary nature of the quantitative and qualitative phases of the study. As Morgan (1998) described, sequential designs focused on complementarity of methods allow the strengths of both methods to be utilized in a coordinated fashion in order to gain a comprehensive understanding of the phenomenon of interest. For this particular study, the principal component was qualitative due to the need to collect nuanced and contextual descriptive information, as well as the lack of appropriate quantitative tools. The preliminary quantitative phase produced information crucial to providing context and relevant information for sampling. The preliminary quantitative phase ensured that those participants included in the principal qualitative phase were best poised to address the research questions of interest. Furthermore, this phase allowed the identification of a sample of programs that vary in compliance. The principal qualitative phase was necessary to gain in-depth descriptive information regarding program representatives' experiences, perceptions, and needs. The qualitative portion allowed for the collection of comprehensive, contextual information regarding the process of implementation (Snape \& Spencer, 2003). Thus, the preliminary quantitative portion (Phase One) provided insight into $R Q 1$ while also providing the basis for the purposive sampling of programs in 
Phase Two. The principal qualitative portion (Phase Two) allowed for an in-depth inquiry into program representatives' responses and reactions to state standards, thus addressing $R Q 2, R Q 3$, and $R Q 4$. While qualitative data collection techniques were employed in Phase Two, analytic procedures were utilized in order to provide a quantitative and qualitative understanding of the interview data. The use of both quantitative and qualitative analytic approaches allows for the comparison of experiences across participants, as well as a deep understanding of individual experiences.

\section{Phase One}

Participants. Phase One participants include program representatives of batterer intervention programs (BIPs) in the state of Oregon. While program directors were initially asked to participate due to their role in overseeing all aspects of program functioning, at times other representatives completed the survey as directed by the program director. Specifically, program representatives were program directors or key program staff members nominated by the program director. In total, program representatives from 47 programs were eligible to participate in Phase One of the study. Of the 47 eligible participants, 35 program representatives completed Phase One, indicating a 74\% response rate. Each participant represented one BIP in Oregon.

Procedure. In order to complete Phase One of the study several steps were required. First, the entire known population of BIPs in Oregon as of $2008(N=59)$ was contacted in the fall of 2011 to establish which programs were still providing BIP services. In order to accomplish this each BIP listed in the 2008 Oregon BIP Directory, which lists the contact information for all known BIPs in the state of Oregon that existed 
in 2008, was contacted via telephone by the researcher. Contact was attempted for all 59 programs listed in the 2008 Oregon BIP Directory. Discussion with directors, probation departments, and other agencies in the county, revealed that 12 programs were no longer offer BIP services. Further, through discussion with staff from the remaining programs it became evident that two programs that had previously been described as distinct programs were actually overseen by the same director and should be considered one program rather than two. Additionally, a representative from one program indicated that BIP services had never been offered by the program and requested their name be removed from the directory.

During this initial contact the researcher verified basic program information (e.g., name, address, phone number, etc.). Programs representatives were also asked to report any new programs in their area that began providing services since the Oregon BIP Directory was last updated in 2008. The newly identified programs were contacted and if the program director indicated that the program provided BIP services, they were invited to participate. This additional step allowed for integration of snowball sampling such that the entire population of programs, including those previously identified in 2008 and newly identified programs, were invited to participate. This snowball sampling technique identified three new programs in the state. This process resulted in 47 known BIPs in the state of Oregon as of fall 2011.

The basic program information gathered through the initial phone contact was compiled in order to update the Oregon BIP Directory. Once updated contact information was obtained, program representatives were informed about the upcoming survey and 
asked if they would prefer to complete the survey on a web-based platform, on paper, or via telephone. Preferences were recorded and utilized during survey administration so that each program was provided with a survey that matched their preferences. During this phone contact, programs were also asked whom the best person would be to direct the survey to in order to ensure the program director received the survey, though in some instances the program director elected to have an informed staff member complete the survey.

Next, a survey was sent to participants via email, mail, or telephone call based on previously indicated preferences. In total, a representative from 30 programs completed the survey on the web-based platform, a representative from four programs completed a paper version of the survey, and a representative from one program completed the survey over the phone. Participants who completed the survey on the web-based platform did so through Qualtrics, a web-based survey program. Participants who completed the survey on paper were mailed the survey along with a self-addressed stamped envelope to return the survey. The participant that elected to complete the survey over the telephone was contacted by the researcher and asked to answer the questions verbally. Program representatives had approximately two months to complete the survey. Survey responses were collected from October $31^{\text {st }}, 2011$ through December $31^{\text {st }}, 2011$.

Measures. Phase One of the study utilized a revised version of the survey administered to BIPs in Oregon in 2008 (see Appendix B). The survey was developed over the course of ten years with the input of various stakeholders. The survey was first created and administered in 2001 by a group of undergraduate community psychology 
practicum students, a university professor, and a BIP director who was involved in the statewide development of the BIP standards. The survey was designed to gain information regarding a variety of program components including the program's philosophical orientation, curriculum and activities, intake and referral procedures, and program fees. Additionally, questions were asked to gauge the amount of collaboration each program had with community agencies, such as victim advocates, probation, and domestic violence councils. Finally, questions were asked to determine characteristics of group facilitators, program length, and completion rates.

The survey was refined and administered for a second time in 2004 with additional input from a BIP director to gain more comprehensive information about program practices and procedures. This version of the survey utilized the same questions as in 2001, with added questions in the form of an addendum. The addendum was created to obtain information about additional characteristics of the programs and more detailed information about several characteristics assessed in the prior survey of the programs. The specific areas examined in greater detail were the nature and extent of contact with victim advocates, the probation department and victims, post-intervention services, and contact with other BIP providers. The survey also asked programs if they were aware of the possibility of state standards and assessed whether or not the program utilized an endorsement process.

The survey was extensively revised and administered for a third time in 2008. The 2008 BIP Survey used a significantly different survey measure than what was used in previous years. This version of the survey was created by the Oregon Attorney General's 
BIP Standards Advisory Committee (Standards Advisory Committee) to examine program characteristics and practices in relation to the recently adopted state standards. The 2008 BIP Survey included both multiple choice and open-ended questions that examine program characteristics relevant to the state standards. This version incorporated questions to assess various program procedures including: intake, referral, transfers, fees, completion requirements, and completion rates. In addition, the survey assessed program length, composition and characteristics of group facilitators, program curriculum and intervention strategies, and program policies. Programs were also asked about how they accommodate the unique needs of their clients (e.g., culture, language, disability), as well as about the services they provide for victims. Finally, programs were asked to comment on their perceived level of compliance with state standards and any barriers experienced while attempting to comply with the standards.

The 2011 BIP Survey was developed utilizing knowledge gained from the previous survey administrations. The survey was revised based on feedback about the administration of the 2008 BIP Survey, as well as through the review of responses provided in 2008. This process was necessary in order to clarify confusing question wording (e.g., clarification of the definition of program length), specify detail, and create structured response options to replace open-ended items. The response options were based on the responses to open-ended items from 2008. This ensured that the content and scope of response options accurately reflect program experiences. However, every multiple-choice item also contained an "other" option to allow for specification of any responses not identified in the 2008 BIP Survey. 
The 2011 BIP Survey content aimed to assess program characteristics relevant to state standards. Questions assessed the following topics: program procedure, intake, referral, transfers, fees, completion requirements, completion rates, program length, composition and characteristics of group facilitators, program curriculum and intervention strategies, and program policies. Programs were asked how they accommodate the unique needs of clients (e.g., culture, language, disability) as well as about the services they provide for victims. Finally, programs were asked about barriers to compliance with standards, as well as the extent to which they believed they were in compliance with standards.

Some questions in the survey directly correspond to components of the standards while others were included to gain more descriptive information about program functioning. Due to the wording of the standards, some aspects of functioning assessed remain relevant to the standards but cannot be utilized to determine compliance. For example, some components of the standards include qualifying language such as, "when possible," which is interesting descriptively but not directly applicable to creating a precise index of compliance. As in prior years, community partners were given ample opportunity to comment on the survey and suggest changes. The 2011 BIP Survey was presented to the Standards Advisory Committee. Feedback was gathered to confirm that the survey made sense in the context of BIPs and the information was useful to the committee.

Analysis. Descriptive statistics were computed to determine the frequency of various program practices and policies. These analyses were directed at answering $R Q 1$, 
which addresses current BIP practices and policies. Program characteristics and policies were not only assessed descriptively, but they were also examined in the context of state standards. Specifically, each aspect of program functioning included in the standards was examined to determine program practices and the percentage of programs that are compliant with that aspect of the standards. Next, an index of compliance was generated in order to differentiate programs based on the extent to which they are compliant with the standards. Compliance was operationalized as the proportion of items relevant to components of the standards to which each program adheres. In total, 38 items were utilized to determine compliance (see Appendix B for the set of questions used to assess compliance). Responses to each item that corresponds with the requirements set forth by the standards were coded dichotomously $(0=$ "not in compliance"; $1=$ "in compliance"). The average of all questions used to compute compliance was taken for each program, resulting in a compliance average for each program that ranges from zero to one, with zero indicating no compliance and one indicating full compliance. In order to generate a compliance average, programs were required to provide valid responses to $75 \%$ of the survey items relevant to compliance (i.e., 27 items). While some missing data did exist, only one program was excluded from the generation of a compliance average. Further, patterns in missing data were examined across high and low compliance programs using the midpoint of compliance such that $50 \%$ of programs fell into the high compliance group and $50 \%$ of programs fell in the low compliance group. While the low compliance programs had more missing data overall, the number of individuals with missing data for each compliance item was similar. This indicates that compliance averages for high 
compliance programs were not falsely inflated due to non-response. The compliance averages were utilized to better understand programs' success with implementation of the standards and to answer $R Q 1$. Additionally, compliance scores were used as a sampling criterion to select participants for Phase Two.

\section{Phase Two}

Participants. Phase Two of the study utilizes the information gained in Phase One to carry out purposive sampling for extreme cases (Ritchie, Lewis \& Elam, 2003; Ryan \& Bernard, 2000). Responses from Phase One were evaluated to determine the degree to which each program complies with state standards. The distribution of compliance averages was examined to determine if there is discontinuity in the distribution that could be used to designate high and low compliance programs. Natural breaks were not identified and therefore the top $20 \%$ of programs in terms of compliance and bottom $20 \%$ of programs in terms of compliance were selected. A tie in compliance scores required the inclusion of one additional program. Thus, seven programs $(20 \%$ of the survey sample) were identified as high compliance programs and eight programs (23\% of the survey sample) were identified as low compliance programs. The program director from each of these programs was asked to participate in Phase Two of the study.

In total, of the 35 programs that completed Phase One, 15 program directors were asked to participate in the Phase Two in-depth interviews and representatives from 13 programs agreed to participate $(87 \%)$. While program directors typically served as the representative for their program in the sample of 13 programs, four program directors indicated that they do not directly manage batterer intervention services and instead have 
a designated staff member responsible for BIP oversight, decision-making, and implementation of standards. Thus, individuals in different roles in these four programs (i.e., assistant director, program manager, and head facilitators) were better poised to address implementation of state standards.

Initially, BIP directors were preferred as participants because it was expected that directors would have the most responsibility for program functioning and would therefore be a more useful key informant (Tremblay, 1957). BIPs are typically structured hierarchically with the program director holding the greatest amount of decision-making power. BIPs in Oregon vary in size and some programs consist of just one individual that serves as the program director as well as the sole facilitator, while other programs employ numerous facilitators and support staff overseen by a director. Many of the requirements set forth in the standards involve structural program characteristics (e.g., written protocol for victim safety; hiring of male and female co-facilitation staff; community collaboration). Program directors are most likely responsible for structural characteristics of the programs, as opposed to staff who are responsible for facilitating individual groups. Thus, it was believed that program directors were best positioned to speak to the effects of the standards and experiences with implementation.

Despite this expectation, conversation with program directors revealed that in some agencies, the program director provides general oversight to numerous programs (e.g., drug and alcohol services; mental health services, etc.) across multiple sites. In these situations it is not uncommon to appoint a staff member to oversee just the BIP component of the agency. Given that some program directors strongly believed that other 
staff members were better suited to discuss the BIP component of the agency, as well as how the standards have been implemented, nominated program representatives were included as participants when appropriate. Specifically, one program director nominated the assistant director, one program director nominated the program manager, and two program directors nominated the primary BIP facilitator. In each case, the nomination was made because the director indicated that while they oversee the organization as a whole, the nominated individual oversees the batterer intervention portion of each organization. The inclusion of those most responsible for the implementation of the standards was vital to ensure that the implementation process and reactions to that process were as thorough and detailed as possible. Additionally, because program directors and key representatives are those responsible for implementation, these individuals are also in the best position to enact change moving forward.

While a representative from each high compliance program agreed to participate in Phase Two of the study, representatives from two low compliance programs indicated they could not participate because of time constraints. These potential participants reported that they did not have staff available to participate, as they served as the single, regular facilitator for their program. These two program directors were asked if they would be willing to participate in an abbreviated interview but both declined. This led to a final sample of representatives from 13 programs for Phase Two of the study $(87 \%$ response rate). These 13 program representatives account for approximately $37 \%$ of Phase One participants. 
Additionally, in order to fully assess the impact of state standards and address $R Q 2 g$, former program directors from the 11 programs that stopped providing BIP services between 2008 and 2011 were contacted via telephone. ${ }^{1}$ From the list of 11 former providers, six (55\%) providers could not be contacted. Of these, three program directors had phone numbers and emails that were no longer in service. Probation departments in each county served by these programs were contacted and new contact information was not available. The remaining three programs that could not be contacted had functioning phone numbers but did not answer phone calls or return messages. Each of these former program directors was contacted a total of two times per week over the course of six weeks via telephone. Messages were left one time per week. Email was attempted for two of the program directors that had previously provided email addresses. Each provider was emailed three times and no email was returned. Finally, probation departments in each county were contacted and new contact information was not available. The remaining five (45\%) former providers were successfully contacted. Former program directors who were successfully contacted were asked to participate in an abbreviated phone interview aimed at exploring the possible impact of state standards for programs that no longer provide BIP services. All successfully contacted program directors agreed to participate (response rate $=100 \%$ ).

\footnotetext{
${ }^{1}$ Of the 12 programs previously identified as no longer offering BIP services, six former program directors were successfully contacted. While speaking with one of these providers, it became evident that that the removal of their program from the directory was a mistake. While this program does not always have BIP groups functioning, they do conduct groups when they have enough clients to do so and wished to be included as a functioning BIP agency. Thus, they were removed from the list of former providers.
} 
Procedure. After the potential current provider participants were selected, the director from each identified program was contacted by the researcher and asked if he or she would be willing to participate in an interview about their experiences adapting to the standards. In some cases $(n=4)$, program directors indicated that they would prefer to nominate a key staff member who is more knowledgeable about the BIP component of the program, as well as the implementation of standards. Program directors or key staff members who agreed to participate scheduled a time for a face-to-face interview. The researcher traveled to each program that agreed to participate and conducted a face-toface interview with the designated participant in a location selected by the participant. Every participant elected to hold the interview in the location where they typically provide services.

While focus groups with program representatives could have also been a viable option for gathering information regarding program representatives' experiences, as the interactive nature of focus groups would allow discussion and idea sharing (Morgan, 1996), they were not the ideal avenue for collecting data for this subject matter. Interviews were selected over focus groups due to the sensitive nature of disclosure related to aspects of compliance. Because these programs rely on and compete for referrals to stay in business, participants may not have been willing to discuss aspects of the standards with which they disagree or fail to comply with due to fear they may experience negative repercussions if others were to learn of this information. This may be especially true in a focus group comprised of providers that potentially compete for referrals with one another. While the standards are not formally monitored or enforced, 
participants' may feel pressure to present their programs in the best light possible if they cannot be assured confidentiality. This concern, as well as the logistical considerations of a statewide sample, made individual face-to-face interviews a logical choice. Further, individual face-to-face interviews were selected due to the intense nature of the interview experience and need for physical proximity in creating a context that allows rapport to be built and participants to share their experiences confidentially without fear of negative consequences (Legard, Keegan, \& Ward, 2003). It is important to note that at the conclusion of the interview participants had the opportunity to identify any aspects of the discussion that they would like to be excluded from analyses. When asked this after completing the interview, the only information that participants requested be removed included names and other identifying information. Beyond these minor modifications, immediately after the interview, participants reported feeling comfortable with their interviews being included in the study.

Across the 13 participants, interviews ranged from approximately 80 to 152 minutes in length. In other words, the shortest interview lasted one hour and twenty minutes and the longest interview lasted two hours and thirty-two minutes. On average interviews lasted 113 minutes (i.e., 1 hour and 53 minutes). This allowed time to fully address each question of interest. To ensure structure, which allows for comparison across interviews, as well as flexibility to permit the discussion of emergent topics, interviews were semi-structured as recommended by Legard and colleagues (2003). The use of a semi-structured interview guide provided consistent questions across 
participants, while maintaining space to assess the unique perspective of each participant. The interviews were audio recorded for subsequent transcription.

After each interview was completed, the audio recording was transcribed by one of three trained undergraduate research assistants. This was completed with the help of a transcription foot pedal and Express Scribe software. Time required for transcription ranged from approximately nine hours and fifteen minutes to fifteen hours per interview. On average, transcription required twelve hours and thirty minutes per interview. This process produced 13 transcripts, which ranged from 24 to 36 single spaced pages of text. On average transcripts were 26 single spaced pages in length.

After transcription was complete, the researcher carefully reviewed each transcript to identify information that may reveal participant identities and address transcription errors or questions from transcribers (e.g., verification of acronyms used by participants with which transcribers were not familiar). Any identifying information was replaced with a generic term in order to maintain meaning. For instance, when a person's name was used it was replaced with a generic title for that individual's position (e.g., 'facilitator', ‘judge', or 'local advocacy organization'). Once identifying information and errors were edited from transcripts, each transcript was distributed to the participant in order to allow the opportunity for participants to review the transcript and determine if there are any portions of the interview they wanted excluded from analysis or if there are any aspects of the interview that may disclose their identity. These steps were included to ensure that participants felt comfortable with the data included in subsequent analyses, as well as to ensure that all possible identifying information was successfully disassociated 
with the content of the interviews. After this review process, eight participants $(62 \%)$ were comfortable with their transcripts in their current form, two participants (15\%) requested slight changes and submitted additional detail to add to their responses, and three participants $(23 \%)$ noted occurrences of and suggested generic terms for identifying information the researcher did not realize was identifying.

Finally, contact was attempted for the former program directors from the programs no longer providing BIP services via telephone. Phone interviews were selected due to the relatively brief nature of the interviews and the statewide sample. Participants were administered a modified and structured version of the full interview guide. Structured interview questions were utilized to provide consistency among phone interviews, comparability between current and former provider interviews, and to ensure the interview did not exceed the time allotted as these participants had responsibilities unrelated to BIP standards. Former program director interviews lasted 28 to 47 minutes in length $(M=37$ minutes). These interviews were not recorded and instead the researcher took detailed notes, including direct quotes when possible, during the discussions. This process produced five sets of interview notes, which ranged from two to three pages of single spaced material ( $M=3$ pages) directly relevant to the questions asked during the interview. Discussion of topics outside the realm of the interview questions (e.g., introductory conversation, comments about the field of batterer intervention broadly, etc.) were not included in the interview notes as they did not pertain to the research questions at hand. 
Measures. The researcher came to interviews with current program representatives prepared with approximately three broad structured interview topics with numerous corresponding sub-questions to generate discussion (see Appendix C). These topics are comprised of interview questions that correspond with the analytic lens used to analyze the data (see Figure 2). Specifically, these questions assessed perceived and actual control, negative attitude change and maintenance, absoluteness, legitimacy, and policy implementation. While interview questions were prepared, the interview itself included aspects of unstructured interviews and thus as a whole can be considered semistructured in nature (Legard et al., 2003). The integration of structured and unstructured interview techniques into a semi-structured interview protocol allowed all topics relevant to understanding policy implementation to be introduced. This also permitted conversation to evolve, allowing the possibility for new topics to emerge (Fontana \& Frey, 2000). Because the current study aimed to examine commonalities and differences across participant experiences, it was important to incorporate similar questions into each interview. While this was important, the goals of the current study called for flexibility to fully examine emergent topics and possibly introduce such topics into subsequent interviews.

With this in mind, the researcher presented questions for discussion and utilized an interactive process to probe the participant for more detailed information as the discussion evolved (Legard et al., 2003). Probes were amplificatory, explanatory, and/or clarifying in nature, depending on the context of the interaction (Legard et al., 2003). Further, if interesting topics arose in previous interviews, the interviewer was permitted 
to introduce new and/or different questions into subsequent interviews, thus inviting an ongoing generation of knowledge (Legard et al., 2003). As interviews were conducted, the researcher paid particularly close attention to the discussion content to identify topics of importance, uniqueness, or interest. This information was used to generate more probes that were added to subsequent interviews based on the judgment of the researcher. For example, after the first interview with a participant located in an area with local standards in addition to state standards, the researcher was careful to include probes to differentiate experiences with local and state standards for subsequent interviews with participants in that particular area. While there was some variation across interviews, the structured elements of the interview consistently engaged participants around numerous topics.

First, participants were asked about their experiences related to the creation and introduction of the standards, as well as their current response towards the standards. Participants were asked to describe how they learned about the standards and their feelings about the creation of standards. They were then asked to discuss their initial reaction to the standards and how their reactions have changed over time. Participants were also asked about the extent to which they contributed to the development of the standards. Finally, they were asked to describe their current understanding of the standards. This information aided in determining how the mandate of standards was delivered across programs. Questions relating to this topic provided insight into whether participants were aware that standards were being developed and whether they were able to provide input into the content. Together, this information provided knowledge 
regarding experiences of actual and perceived control. It was expected that differences in awareness of and contribution to the standards creation process might offer knowledge regarding how participants perceive the standards, as well as the extent to which they are able to comply with standards. Further, discussion related to the various responses to the introduction of the standards provided information regarding the extent to which participants changed or maintained initial negative attitudes towards the standards. Finally, learning whether participants view the way in which standards were dictated by state policy as appropriate or inappropriate provided insight into the perceived legitimacy of the standards.

Second, participants were asked to describe their unique process of implementation since they learned about the standards. This included describing their own understanding of the content of standards, which practices their program had to change, how they made those changes, and which aspects of their program were able to remain unchanged. Together, this provided information useful to determining the extent to which program representatives clearly understand the standards and the process by which the policy has been implemented in BIPs. To fully understand implementation, it is necessary to have knowledge of all available resources. Participants were asked to detail any support they received in implementing the standards. Support may take many forms (e.g., financial, personnel, training, collaborations) and program representatives were asked to identify anything that has enabled compliance. In addition to the sources of support available to programs, participants were also asked to describe any other resources that have enabled their program to comply with state standards. This 
information was useful for understanding what facilitates key program staff members' ability to implement various components of standards.

Understanding the barriers to compliance is as important as understanding the enablers to compliance. Participants were asked to discuss any barriers they have faced in implementing the standards. Previous work investigating BIPs in Oregon identified nine types of barriers to compliance with the standards reported by BIP directors (Boal \& Mankowski, in press). These barriers include: difficulty finding facilitators; lack of funding; difficulty meeting training requirements; rural location; time and workload difficulties; hardships in creating and maintaining necessary collaborations; inability to accommodate client needs; lack of evidence based requirements; and conflict with county requirements (Boal, 2010). This information was vital for ensuring that the full range of potential barriers was discussed in the interviews. Specifically, participants were asked to describe barriers they face, how they have overcome barriers, and which barriers they have not yet been able to overcome. Probing questions were applied based on the previously identified program barriers.

Third, participants were asked to specify their perceptions towards the standards. Specifically, they were asked to describe the extent to which they endorse the idea of state standards for BIP practice, as well as their experiences related to compliance. This line of questioning provides insight into how program representatives think about standards in general. Specific issues that were raised when discussing this topic included the need to control BIPs versus BIP independence, and the value of uniformity versus allowance of variation in programs. In addition to attempting to understand participants' 
attitudes about the standards, their thoughts about specific components were assessed. Participants were asked to describe specific components of the standards they believe should or should not be included in standards, as well as their own program practices that do and do not correspond to standards. This information was used to determine where there is consistency between the standards and the preferred practices of providers. This topic was included in order to gain insight into the extent to which negative initial attitudes towards the standards were changed or maintained, and perceptions of policy logic.

As standards are not currently monitored or enforced, it is important to have a thorough understanding of whether participants feel pressure to comply with the standards, as well as the degree to which they believe the mandate of standards is absolute. This line of questioning asked participants to speak to the degree to which they feel compliance is expected and necessary, and why. If there are any local monitoring sources, they were asked to describe them and the process by which their compliance with standards is assessed. Participants' were also asked to discuss whether the process by which they receive referrals has been altered because of the standards. This provided the opportunity to gain further information about the occurrence and extent of informal monitoring and enforcement. Items included in this line of questioning assess whether standards are viewed as absolute, along with norms regarding compliance. Providers were also asked to describe the extent to which they believe they can impact the standards in order to gauge perceived control. 
The topics described above were selected in order to provide a comprehensive depiction of the ways in which participants have navigated and thought about the implementation process. The information gathered from these interview topics provides a rich and detailed understanding of how participants have reacted and responded to standards. The interview guide included all interview questions according to their sequence in the interview and is based on the three areas of inquiry described above (Appendix C).

Additionally, a modified structured interview guide was utilized for the phone interviews that were conducted with former BIP directors (see Appendix D). This interview guide employed a funnel approach (Morgan, 1997). Specifically, the interview began with a broad assessment of the reasoning behind no longer offering BIP services and then become more specific and asked former providers to reflect on the impact of standards. This interview guide incorporates the most relevant questions from the larger interview guide in order to allow consistency in coding protocol across the interviews with former and current program representatives. Further, this ensures that the same social psychological theoretical lenses could be applied to the information provided by former program directors.

Analysis. Phase Two analyses were directed at answering $R Q 2$, which asks about program representatives' responses to Oregon BIP standards; $R Q 3$, which asks about participants' experiences with implementation of the standards; and $R Q 4$, which asks about the extent to which representatives from high and low compliance programs differ and the nature of those differences. Analyses were conducted utilizing a thematic analysis 
approach that incorporates predetermined and emergent themes. This approach was selected due to the researcher's desire to examine the extent to which established social psychological theory and the policy implementation literature helps explain program representatives' responses to state standards, as well as to ensure responsiveness to unexpected or novel themes that arise. After the completion of the coding process, the number of instances of each code, as well as the content of coded material was incorporated in analyses.

Data preparation and coding consistency. In order to carry out qualitative thematic analyses of the interview data, several steps were taken. This process followed data analysis recommendations typical to thematic analysis (Ritchie, Spencer \& O'Connor, 2007). First, the researcher developed an instruction manual to guide transcription. This manual outlined the desired format of the transcribed documents, conventions regarding patterns in human speech and how to capture those nuances within a transcript, and procedures for addressing unclear or ambiguous speech. This manual was provided to the undergraduate research assistants responsible for transcription. Each research assistant was provided training regarding the content of the manual, the use of the Express Scribe software, and the use of a transcription foot pedal. After training was complete, the research assistants carried out transcription under the guidance of the researcher. The researcher was responsible for reviewing each transcript, as well as listening to inaudible or unclear audio segments in order to ensure transcriptions were accurate. As each transcript was completed, the researcher closely inspected the transcripts in order to remove identifying information, correct typos, and absorb the 
content of the interviews. This process led to clarification and modification of the predetermined coding system to better capture important aspects of participant experiences. This process was consistent with that of familiarization described by Ritchie and colleagues (2007). While several new codes were added (e.g., social action research, discussion, limited contact) and a small number of pre-existing codes were modified, by and large, the predetermined coding system aligned with the content of the interviews in relation to the research questions proposed by the current study. Specifically, three new codes were added and two sets of code pairs were condensed into one.

The selected codes were as clear and explicit as possible in order to ensure an objective coding scheme (Smith, 2000). Codes were applied to any word, phrase, sentence, or paragraph that was reflective of the code's meaning. Variation in possible coding units was possible due to the varied complexity of different codes. For instance, coding of experiences related to social psychological constructs often required longer segments of text, while coding of implementation experiences could be very brief. Thus, the coding procedure permitted codes to be applied to segments as long or short as deemed necessary by the coders. All coding was initially completed on paper printouts of the transcripts utilizing highlighters and pens of various colors to denote different codes. Paper transcripts were utilized initially due to the preferences of the coders. Specifically, the coders felt they were more comfortable reading the large amount of interview data via a paper rather than computer medium. After the paper transcripts were coded, these codes were entered into the computer program Atlas.ti (Atlas.ti, Version 7) for analysis. The researcher primarily completed entry, except in the case of the former provider 
interviews. For these interviews, one of the coders applied the relevant codes from the paper transcripts into Atlas.ti.

In order to ensure consistent and accurate coding, several steps were taken. First, the researcher provided approximately six hours of detailed training about the codebook and coding procedures. Specifically, the coders were provided with academic articles (Bartholomew, Henderson, \& Marcia, 2000; Hsieh \& Shannon, 2005; Morgan, 1993; Elo \& Kyngas, 2008; Ritchie et al., 2003) describing the coding process, as well as the codebook. The process of coding was discussed in the context of the articles in order to ensure the procedures for coding aligned with normative procedures for qualitative coding. The codebook was discussed in detail in order to provide a concrete definition and example of each code, as well as address any questions about definitions raised by the coders. Next, the two coders and the researcher attempted to apply the coding system to one interview transcript. After coding was complete, the coders and researcher met for approximately seven hours to review the codes applied, as well as the rationale for each code applied. This process provided the opportunity to identify discrepancies in interpretation and application of codes. Further, discussion led to minor modifications of the codebook to clarify wording. This process ultimately yielded one transcript that was coded through consensus.

This process was then repeated with a second interview transcript. Specifically, the researcher and the two coders each applied codes independently and then met to discuss the coding. This transcript was discussed for a total of five hours. While each coding choice was discussed to ensure consistency in understanding of the code 
definitions, this transcript revealed greater consistency among coders. After extensive discussion and the completion of coding through consensus for this transcript, it appeared that the codebook was exhaustive and there was a shared understanding of the coding system and the codebook. Thus, the coders were permitted to begin coding the remaining transcripts independently.

In order to assure interrater agreement, Cohen's kappa (Cohen, 1960), a widely used measure of agreement, was utilized. Due to its ability to account for chance agreement among coders, Cohen's kappa is a more commonly used measure of interrater agreement than percent agreement, which does not factor in chance agreement (Bakeman, 2000; Bartholomew et al., 2000; Crano \& Brewer, 2002). Additionally, Cohen's kappa was selected because of the limitation inherent in utilizing percent agreement- the frequency of any given code will impact percent agreement (Smith, 2000). While this index of agreement only provides an overall index of agreement, it has the benefit of permitting examination of agreement matrices can be utilized to identify problematic codes if necessary (Bakeman, 2000).

Cohen's kappa was calculated after approximately one quarter $(n=4)$ of the interviews were coded independently in order to determine if there were any issues in the coding process that were not identified in the preliminary coding process. This preliminary check identified several problematic codes, as evidenced by kappa below .50 . As recommended by Weick (1985) the codes were examined to determine if the codebook should be revised and/or the coders should be retrained. This led to the identification of four codes that were being applied inconsistently across coders. 
Specifically, the codes of positive current response, negative current response, alignment with standards, and misalignment with standards were problematic. After reviewing the material to which these codes were applied it became evident that the coders were having a difficult time differentiating positive current response and alignment with standards, as well as negative current response and misalignment with standards. After further reviewing the purpose and definition of these codes it became evident that while the researcher had initially conceptualized them as distinct, with one created in the context of social psychological phenomenon and one in the context of policy implementation, they were actually aimed at gathering the same information. Thus, these codes were condensed so that alignment with standards was incorporated into positive current response and misalignment with standards was incorporated into negative current response. Material already coded with the previous coding system was combined so that just the current response codes were utilized and subsequent coding was based on the new definition of positive current response and negative current response. Other codes with a kappa at or below .60 were discussed with coders in order to improve training and clarify understanding so that these kappas would not decline. After adjustments were made, coders continued the coding process.

Cohen's kappa was again calculated after all transcripts were coded to determine final interrater agreement (see Table 1). Final kappas ranged from .55 to .1.00, with only two kappas falling below .60. In total, kappa for 9 codes can be considered excellent $(\boldsymbol{\kappa}=$ .75 or greater), and kappa for the remaining 19 codes can be considered good $(\kappa=.40$ or greater) (Banerjee, Capozzoli, McSweeney \& Sinha, 1999). In addition to determining 
that the application of codes was done reliably, it is also necessary to fully understand the analytic and coding procedure used in Phase Two of the current study. The following coding and analytic strategies allowed for the distillation of the vast interview data into manageable segments in order to address $R Q 2, R Q 3$, and $R Q 4$.

Coding strategy. The final codebook is available in Appendix E. In order to examine $R Q 2$, interview transcripts in their entirety were coded to identify experiences of actual control, perceived control, negative attitude change and maintenance, absoluteness, and legitimacy. Numerous codes and constellations of codes were utilized to assess each of these social psychological phenomena. Specifically, the coding system was implemented to provide data to inform the seven sub-questions included within $R Q 2$, as well as the three hypotheses proposed within this research question.

In order to address $R Q 2 a$ and determine the extent to which participants have experienced actual control over the content and development of the standards, interview transcripts were coded for experiences of high and low actual control. High actual control was operationalized to capture experiences in which the participant has been or currently is actively involved in the creation and refinement of the standards. This included experiences as a member of the Standards Advisory Committee, experiences providing input directly to the Standards Advisory Committee, experiences providing input to a member of the Standards Advisory Committee, and awareness of or familiarity with the process by which standards were created. Conversely, low actual control was coded when participants indicated they were not and currently are not involved in the 
creation or refinement of standards, or they are unaware of the process by which standards were created.

In order to address $R Q 2 b$ and determine the extent to which participants perceive having control over the content and scope of the standards, interview transcripts were coded to identify high and low perceived control. This was achieved through the use of four codes: high perceived ability, low perceived ability, high procedural justice, and low procedural justice. High perceived ability was coded when participants indicated they believe they could have participated in the creation of the standards if they had wanted to, they believe they know who to go to if they have a concern about the standards, or they think that if they brought a concern to the Standards Advisory Committee it would be taken seriously. Low perceived ability was coded when participants indicated they do not believe they could have participated in the creation of the standards if they had wanted to, they are unaware of who to contact if they have a concern about the standards, or they think that if they brought a concern to the Standards Advisory Committee it would not be taken seriously. High procedural justice was coded when participants reported that they believe those on the Standards Advisory Committee represent most providers or they believe that the process by which the standards were created is fair. Low procedural justice was coded when participants indicated they believe that those on the Standards Advisory Committee do not represent most providers or they believe that the process by which the standards were created was unfair. High perceived control was operationalized to include high perceived ability and high procedural justice, while low perceived control was operationalized to include low perceived ability and low procedural justice. 
In order to address $R Q 2 c$ and $R Q 2 d$, which focus on the extent to which negative attitudes towards the standards change (i.e., a proxy for rationalization) or are maintained (i.e., a proxy for reactance), a constellation of several codes was utilized. Interview transcripts were coded to identify positive and negative initial reactions to the standards, and current positive and negative perceptions of the standards. Positive initial response was coded when participants indicated that when they learned of the standards they were in agreement with the concept and/or content of the standards. Negative initial response was coded when participants indicated that when they learned about the standards they disagreed with the concept and/or content of the standards, or when participants described differences between the content of the standards and current or ideal program practices. Next, positive current response was coded when participants indicated they currently agree with the concept and/or content of standards and negative current responses was coded when participants indicated they currently disagree with the concept and/or content of standards, or when participants described similarities between the content of the standards and current or ideal program practices.

In order to assess $R Q 2 e$ and determine the extent to which participants view the standards as absolute, interview transcripts were coded to capture perceptions of the standards as absolute or non-absolute. The absolute code (i.e., absoluteness) was utilized when participants indicated that they believe adhering to the standards is required and/or expected, they describe experiences with others that require compliance, or they have experienced changes in referral sources due to compliance. The non-absolute code (i.e., non-absoluteness) was utilized when participants indicated that they believe adhering to 
the standards is not required and/or expected, or they describe experiences with others that do not require or value the standards.

The next sub-question associated with understanding responses to standards is $R Q 2 f$, which asks whether participants view the standards and process by which the standards were created as legitimate. To address this sub-question, several codes were utilized in order to include the three key factors associated with legitimacy: procedural justice, social norms, and policy logic. The procedural justice codes were applied as was described for perceived control. Specifically, high procedural justice was coded when participants indicated that they believe the process by which the standards were created and are refined is fair, or they believe that those on the Standards Advisory Committee represent the interests of most providers. Low procedural justice was coded when participants indicated that they believe the process by which the standards were created and are refined is unfair, or they believe that those on the Standards Advisory Committee do not represent the interests of most providers. The positive norms code was applied when participants described favorable or positive discussions of the standards with other providers or the perception that those in the BIP community agree with the standards. The negative norms code was applied when participants described unfavorable or negative discussions of the standards with other providers or the perception that those in the BIP community disagree with the standards. High policy logic was coded when participants indicated that they believe the standards have been created based on knowledge of evidence-based practice, best practice, or provider experiences in the field. Low policy logic was coded when participants indicated that they believe the standards 
have been not created based on knowledge of evidence-based practice, best practice, or provider experiences in the field. High legitimacy was operationalized as including high procedural justice, positive norms, and high policy logic. Low legitimacy was operationalized as including low procedural justice, negative norms, and low policy logic.

The final sub-question that was examined in order to understand participants' reactions and responses to state standards is $R Q 2 g$, which asks how state standards have impacted BIP closures across Oregon since 2008. In order to address this question the detailed notes obtained during telephone interviews with former program directors were coded utilizing the codebook developed for current program participants, with the addition of one code pair. Impact codes were included to identify the extent to which former program directors attribute the closure of their program to standard. Specifically, experiences consistent with both high and low impact of standards were coded. High impact was coded if former program directors identified the standards as a primary reason or cause for their program closure. Low impact was coded if former program directors indicated that the standards did not play an important role in their decision to close their program. Next, the content of the telephone interviews was examined to determine whether the codes presented above, including actual control, perceived control, negative attitude change and maintenance, absoluteness, and legitimacy are experienced by former program directors. Each code that occurred was examined as it was for current program participants. Together these codes provide insight into the extent to which former program directors feel the standards played a role in their program shutting down, as well as whether or not any social psychological processes were salient and therefore 
discussed by the former provider. It is important to note that the former provider interview protocol was an abbreviated version of the current provider interview, and consequently the material that was generated was not as extensive, and possibly as a result, not every code that occurred in the current provider interviews occurred in the former provider interviews. Thus, an examination of each social psychological process evaluated for current providers was conducted to the extent possible in the material. In order to examine $R Q 3$ interview transcripts in their entirety were coded to identify the experiences relevant to the policy implementation process. A total of nine codes were applied to better understand policy implementation for program representatives in Oregon. First, $R Q 3 a$ asks what specific strategies have program representatives have used to implement the standards. In order to address this question, the code of implementation strategies was employed. This code includes any descriptions of specific strategies undertaken to begin, continue, or maximize implementation. From this code, a comprehensive collection of strategies that have been employed by participants was generated in order to identify consistencies and variation in implementation strategies.

In order to evaluate $R Q 3 b$, which asks which program policies and characteristics are described as relatively easy and relatively difficult to implement by program representatives, two codes were utilized. The first code, implementation ease, captured any practices for which participants reported they did not have to change, as well as practices that required little effort or resources to implement. The second code, implementation difficulty, captured any components that they have not yet been able to 
change, as well as practices that required a large amount of effort or resources to implement. Together these codes provided a comprehensive view of how programs have or have not shifted due to the standards, as well as how easy or difficult those changes were for the programs.

In order to evaluate $R Q 3 c$, which aims to identify facilitators to implementation or compliance, one code was utilized. The code enablers to compliance was applied whenever a participant described agencies, specific activities, ways in which standards are worded, or program characteristics that enable/encourage compliance. A similar strategy was used to evaluate $R Q 3 d$, which aims to identify barriers to compliance. One code, barriers to compliance, was applied whenever a participant described agencies, specific activities, ways in which standards are worded, or program characteristics that inhibit compliance. The material captured with these codes addressed $R Q 3 c$ and $R Q 3 d$ by generating a detailed list of all enablers and barriers to implementation experienced by participants.

In order to evaluate $R Q 3 e$, which asks what needs participants identify in order to successfully implement the standards, one code was used. Support for compliance was coded whenever a participant describes a resource, relationship, modification to standards, or other source of support to enable compliance. A complete list of the support for compliance suggestions was generated based on this code in order to describe what participants believe would be helpful in achieving greater implementation.

Analytic Procedure. The coding system was utilized to gather detailed descriptions of reactions and responses to the standards relevant to the research questions 
that existed in the data. Descriptive statistics and illustrative quotes were used to answer $R Q 2, R Q 3$, and $R Q 4$. In order to address $H 2 a, H 2 b, H 4 a, H 4 b$, and $H 4 e$, inferential statistics, specifically t-tests, were used in addition to descriptive statistics and content of codes to compare groups. In addition to the coding process that was employed for codes relevant to the predictions of the current study, further interpretation of participants' responses was conducted to provide context for the frequency of the various codes utilized in previous steps of the analysis. This process was replicated to examine the detailed notes from phone interviews and identify responses to the standards for those no longer providing BIP services. Given this broad description of the strategy utilized in the current study, more specific details related to the analytic strategy for each of the research questions are described below.

Research question two. The analytic strategy to address $R Q 2$ involved several steps. First, descriptive statistics for each code were generated to determine the frequency of each code, the range in frequency across participants, the average number of instances of each code, and the percentage of participants who discussed experiences consistent with each code at least once. Second, because each sub-research question and hypothesis involved at least two codes, ratios were computed to determine the prevalence of each code in relation to other pertinent codes within each participant's interview. Specifically, interview transcripts were coded for high and low experiences and perceptions consistent with each of the social psychological phenomenon assessed in order to understand the range and diversity of experiences. Ratios were generated to identify the proportion of high experiences compared to all relevant experiences for a given code. For example, one 
set of codes assesses experiences of actual control by applying a code to capture high actual control and a code to capture low actual control. In order to determine the proportion of experiences related to control that were consistent with high actual control, a ratio was developed. This ratio included the number of high actual control experiences divided by the number of high actual control and low actual control experiences combined. This resulted in a ratio that could range from zero to one, with zero indicating no experiences consistent with high actual control and one indicating experiences of high actual control exclusively. This process was repeated for every grouping of codes in order to capture variation within and between participants. After developing these ratios, descriptive statistics were computed to determine the range, mean, and standard deviation of these ratios. Further, participants were often grouped based on whether their responses were primarily consistent with high or low experiences of a given phenomenon. Finally, the qualitative material related to each code was assessed in detail. This was accomplished through a thorough review of each relevant quote in order to identify similarities and nuances within the content of each code, as well as provide exemplar quotes.

While this analytic strategy was used across all sub-questions pertinent to $R Q 2$, several sub-questions and hypotheses required additional analytic steps. Specifically, the areas that required additional analytic procedures included: $H 2 a, R Q 2 c, R Q 2 d, H 2 b$, and $R Q 2 f$. After the appropriate codes relevant to each of these research questions and hypotheses were applied, subsequent steps were taken to fully address the question or prediction at hand. 
After experiences of actual control and perceived control were coded, $H 2 a$ was evaluated. $H 2 a$ states that those who report actual control over the standards will describe higher perceived control as compared to those that do not report actual control. In order to assess this hypothesis, the frequencies of all relevant codes were examined, along with the actual control and perceived control ratios. Actual and perceived control ratios were compared to identify patterns in perceived control ratios among those who have actual control ratios above and below .50. A t-test was conducted to determine if the perceived control ratios were significantly different among participants who primarily reported having actual control versus participants who primarily reported the absence of actual control. In addition to evaluating the number of instances of each code within the interview data, the specific content of responses was also evaluated in order to provide context and depth, as well as determine whether the content of the responses aligns with the quantitative findings.

After codes relevant to $R Q 2 c$ and $R Q 2 d$ were applied, additional steps were taken to identify and assess the extent to which attitudes towards the standards change (i.e., a proxy for rationalization) or are maintained (i.e., a proxy for reactance). First, the scope of these experiences was examined. This was accomplished by examining each code in isolation using the number of instances each code was mentioned and the specific content of coded material, as well as computing the ratios for initial response and current response. Once these experiences were described, each interview was evaluated to determine the pattern of responses. Specifically, those who were coded as having a primarily negative initial response (initial response ratio $\leq .50$ ) were examined to 
determine if their current response is primarily positive or negative. Transcripts were coded as shifting initial negative attitudes (i.e., a proxy for rationalization) if the participant had a primarily negative initial response and a primarily positive current response (current response ratio $>.50$ ). Transcripts were coded as maintaining initial negative attitudes (i.e., a proxy for reactance) if the participant had a primarily negative initial response and a primarily negative current response (current response ratio $<.50$ ). Further exploration into the change or maintenance of initial negative attitudes towards the standards occurred after the absoluteness codes were taken into account through the assessment of $H 2 b$. Hypothesis $2 b$ states that program representatives who respond to the standards with rationalization (i.e., retrospective report of changing initial negative attitudes) will view the standards as more absolute than program representatives who respond to the standards with reactance (i.e., retrospective report of maintaining initial negative attitudes). In order to evaluate this hypothesis, the absoluteness ratio was evaluated for those who changed their negative attitudes and those who maintained their negative attitudes to determine if patterns in absoluteness ratios among these groups existed. A t-test was conducted to determine if the absoluteness ratios were significantly different among participants who changed their negative attitudes versus participants who maintained their negative attitudes. Next, the content and depth of qualitative responses regarding absoluteness for the negative attitude change and maintenance groups was assessed to identify consistencies and divergences in experiences of absoluteness among these groups. The evaluation of this hypothesis provides insight into the extent to which absoluteness differs among those who changed their initial negative attitudes towards the 
standards (i.e., a proxy for rationalization) and those who maintained their initial negative attitudes towards the standards (i.e., a proxy for reactance).

Finally, after codes relevant to legitimacy were applied additional steps were taken to address $R Q 2 f$. After exploring each code in isolation, legitimacy scores were assigned. Specifically, a legitimacy ratio was computed to capture the proportion of high procedural justice, positive norms, and high policy logic experiences out of all relevant experiences. This produced a legitimacy ratio that ranged from zero to one, with zero indicating no experiences consistent with legitimacy and one indicating experiences consistent with legitimacy exclusively. After this ratio was established using the consolation of the six legitimacy related codes, these experiences in combination were assessed qualitatively.

Research question three. Fewer steps were required to address $R Q 3$ among interview participants. Specifically, the computation of ratios was not necessary. To address this research question descriptive statistics for each code were generated to determine the frequency of each code, the range in frequency across participants, the average number of instances of each code, and the percentage of participants who discussed experiences consistent with each code at least once. Second, the qualitative material related to each code was assessed in detail. This was accomplished through a thorough review of each relevant quote in order to identify similarities and nuances within the content of each code, as well as provide exemplar quotes. The content of these codes was utilized to segment responses into different categories and determine similarities and differences across implementation experiences. 
Research question four. Finally, in order to examine $R Q 4$, which asks the extent to which the implementation strategies and responses to standards differ for high and low compliance program participants, codes that were previously identified throughout the interview transcripts were utilized. For each sub-question the frequency and mean instances of each relevant code, as well as the percentage of participants who reported experiences consistent with each relevant code at least once, were examined in the high and low compliance groups. Next, relevant ratios were examined to determine if the ratio of responses differed descriptively across the two compliance groups. Qualitative explorations were also conducted to examine the content and depth of responses within the two compliance groups for the phenomenon at hand.

$H 4 a$ predicts that high compliance program participants will report greater experiences of actual control as compared to low compliance programs. This hypothesis was assessed by evaluating material coded as high actual control and low actual control as it corresponds to compliance level. A t-test was conducted to determine if the actual control ratios were significantly different among the high and low compliance groups. $H 4 b$ predicts that high compliance programs will report greater experiences of perceived control as compared to low compliance programs. This hypothesis was addressed by examining material coded as high perceived control and low perceived control as it corresponds to compliance level. A t-test was conducted to determine if the perceived control ratios were significantly different among the high and low compliance groups. $H 4 c$ predicts that high compliance program representatives will describe relatively more reactions consistent with change in negative attitudes towards the standards (i.e., a proxy 
for rationalization) as compared to low compliance program representatives. This hypothesis was evaluated by examining the relevant codes (e.g., positive initial response, negative initial response, positive current response, and negative current response) and distribution of participants identified as initially having negative attitudes towards the standards and shifting attitudes to be primarily positive in $R Q 2 c$ across the high and low compliance groups. $H 4 d$ predicts that low compliance program representatives will describe relatively more reactions consistent with maintenance of negative attitudes towards the standards (i.e., a proxy for reactance) as compared to high compliance program representatives. This hypothesis was evaluated by examining the relevant codes (e.g., positive initial response, negative initial response, positive current response, and negative current response) and distribution of participants who were identified as having maintained their negative attitudes towards the standards in $R Q 2 d$ across the high and low compliance groups. $\mathrm{H} 4 \mathrm{e}$ predicts that high compliance program participants will report greater experiences of legitimacy as compared to low compliance programs. This hypothesis was evaluated by examining the relevant codes (e.g., high procedural justice, low procedural justice, positive norms, negative norms, high policy logic, and low policy logic) and distribution of legitimacy ratios across the high and low compliance groups. A t-test was conducted to determine if the legitimacy ratios were significantly different among the high and low compliance groups.

\section{Role of the Researcher}

The researcher played a prominent role from the inception of the study, as well as throughout Phase One and Phase Two of the current study. Before the study began, I 
applied for and secured the Policy Initiative Grant from the Society for Community Research and Action (Division 27 of the American Psychological Association) to support this project. After securing funding for the current project, I was responsible for all major tasks, as well as all training for those working on the study. Specifically, I was the primary person responsible for instrument development, outreach to participants, data collection, and data analysis, as well as follow-up and correspondence with participants.

Because I played a central role in the current study, my previous exposure to the Oregon intimate partner violence (IPV) community should be noted. My personal involvement with several key stakeholders may be integrated into multiple aspects of the study, including the study's design, interview topics, data analysis, and interpretation. This study was conducted in collaboration with the Oregon Attorney General's BIP Standards Advisory Committee. Through the aforesaid collaboration, I have become acquainted with many individuals who contributed to formulating the standards, as well as their ideas and beliefs about the content of the standards. This knowledge may impact how I view the standards and specific components of the standards. I have become aware of the rationale behind various components of the standards, as well as debates within the Standards Advisory Committee that have occurred related to some topics (e.g., length). This knowledge has provided me with additional context as to which components seem to be agreed upon within the Standards Advisory Committee and have an agreed upon justification, as well as the components about which there is less consensus. Further, for approximately three years I served as a volunteer note taker during meetings of a regional association of BIP providers. In this role I attended the monthly meetings with providers 
from several counties and was able to listen to their thoughts and experiences as service providers. Participation in these meetings alerted me to the differences in local standards that are used in some areas of Oregon. I heard a great deal of conversation about the use of local standards and the problems providers perceived related to their use. This may have made me more keenly interested in the perspectives of those who are most familiar with local standards as I conducted the interviews.

These experiences may have affected how I conducted the interviews and analyzed the data for this study because I have familiarity with the challenges providers face, the impact some components of the standards have had on their programs, and concerns from other members of the community collaborative response to IPV. My experiences with providers at these meetings have typically been positive. In these meetings it seems as though most providers are trying very hard to do their best work, encourage their clients to change, and make the community a better place. This likely created some appreciation for the work that they do and may have made me more understanding of areas of non-compliance when I considered the context that these providers work within. While this may impact my perceptions towards providers, it is important to recognize that this likely improved rapport during the interviews and created trust with providers, as I was genuinely interested in hearing their stories. While experiences with providers may have made me more sympathetic towards their experiences, I have also had positive interactions with other members of the community collaborative response including representatives from the criminal justice system and victim advocacy organizations. These interactions made me more keenly aware of the 
components of standards that are most salient to those outside of the batterer intervention field, as well as the concerns about program practices without standards. Specifically, I became aware that for some in the victim advocacy community maintaining program length, ensuring facilitators are adequately trained, and having successful collaborative relationships is extremely important, while some individuals from the criminal justice system questioned the need for 48 weeks of intervention. These experiences likely made me more attuned to conversation about the advantages and disadvantages of program length, the reasons that programs had not fulfilled the training requirements, and the challenges and successes encountered when establishing and maintaining collaborative relationships. While I may have been more attuned to these areas, it is valuable to recognize that these are areas of interest and discussion among the professional community. Further, the use of objective coders removed any potential bias that I may bring from the coding process.

Finally, this project is not my first exposure to the study of BIPs and standards. My master's thesis project examined BIP practices and characteristics in relation to state standards. Because of my work on this previous project, I have some knowledge about components of standards that were more or less difficult for programs to achieve, as well as knowledge of the barriers to compliance they reported. This knowledge could have potentially impacted the way in which I ask questions and interpret responses. One area that I was more acutely attuned to during the interviews was that of community collaboration. I was aware that community collaboration tended to be a barrier described by participants (Boal \& Mankowski, in press). Because of this, I typically used probing 
questions to learn more about participant experiences related to community collaboration. While this may have made the interview more guided toward this topic, it did appear to be salient as most participants had a great deal to say about the positive and negative aspects of community collaboration.

Thus, while my experiences with the Standards Advisory Committee and BIP providers could be interpreted as biases, these experiences also provide me with a great deal of context and access to participants. These interactions alerted me to debates in the field and assisted in identifying important questions to address. Moreover, these experiences have allowed me prolonged exposure to the BIP community, which may have fostered participants' trust in myself as a researcher and willingness to participate among providers. Given my familiarity with the Oregon BIP and IPV community, I played a prominent role in all aspects of the study, which are described in detail below.

Phase One. During Phase One, I completed several critical steps to ensure as many programs as possible participated in the survey. First, I created the 2011 BIP Survey by adapting the 2008 BIP Survey and obtaining feedback from the Standards Advisory Committee. The 2008 BIP Survey included numerous open-ended questions. In order to provide consistent response options, I reviewed the range of responses provided in 2008 and developed response options that reflected 2008 responses. Additionally, I modified items that participants did not understand in the 2008 BIP Survey, as evidenced by responses that did not make sense. After making these modifications, I presented the survey to the Standards Advisory Committee for input and feedback. This process 
provided several minor suggested revisions to clarify wording and ensure the survey made sense to providers. Once the survey was prepared, I began contacting programs.

I contacted a representative from each program via telephone and/or email in order to obtain updated contact information and inform them that the survey would be distributed shortly. This required numerous phone calls and emails that took place over the course of several months. Specifically, initial contact began in September 2011 and I continued to attempt contact for non-responsive programs until the survey submission period closed in December 2011. In addition to calls to each BIP, I also contacted other relevant agencies to try to get in touch with non-responsive programs. These included inquiries made to other BIP providers, probation departments, and DV councils. The information gathered through this preliminary process ensured that contact information was correct, participants were informed about the survey and able to anticipate its arrival, and the most appropriate distribution choice (i.e., web, paper, or phone) was known for each program. Further, this information was used to update the Oregon BIP Directory. Two undergraduate research assistants working under my direction completed the directory update task.

After initial contact was complete, the 2011 BIP Survey was administered. I distributed the survey to most programs via web using the emails previously acquired through the initial contact. A subset of programs requested the survey be administered via paper copy. For these participants I mailed a hard copy survey, along with an addressed, stamped envelope for survey return. One participant requested to complete the survey over the phone. I scheduled an appointment with this participant and read the survey 
aloud over the phone and recorded responses. In addition to survey administration, I took several steps to encourage participation. This included regular email and phone reminders, as well as an announcement at a local BIP providers meeting. These reminders took place over the course of three months, starting in September 2011 and concluding in December 2011.

After the survey window closed, I was responsible for all data entry and cleaning. I supervised two undergraduate research assistants to complete data entry for the paperand phone-administered surveys. This included providing training on the use of SPSS and oversight during the data entry process. One research assistant entered each interview into SPSS and the entry was double checked by a second research assistant to ensure accuracy. I addressed any discrepancies or questions that arose. Following data entry, I performed extensive data cleaning within the SPSS database to ensure the database included clear and accurate coding of the data. Finally, data analysis was conducted. I was responsible for all analyses to address $R Q 1$ and identify high and low compliance programs necessary to proceed to Phase Two.

Phase Two. After identifying potential participants for Phase Two of the current study, I was responsible for soliciting participation and carrying out all data collection and subsequent analysis. First, I contacted each potential current program participant to inform him or her about Phase Two of the study and ask if they would be willing to participate. This process began in early May of 2012 and continued through mid-June 2012. This typically required numerous phone calls and emails to get a hold of the participant and schedule a time to meet. Additionally, I contacted former providers who 
had ceased providing BIP services between the administration of the 2008 and 2011 BIP Surveys. Some of the former providers were extremely difficult to contact due to the their absence from the community of providers for several years. In order to identify former program participants who had inactive numbers or emails, or would not respond to my messages, I called other relevant agencies. This included probation offices, other BIP providers, and advocacy agencies. This process began in November 2012 but after limited success, calls were stopped and then resumed after the holiday season. Calls began again in January 2013 and continued through February 2013.

After securing a scheduled meeting with current provider participants, I traveled to each participant's chosen interview location. Interviews were held across the state of Oregon and in order to conduct these interviews I drove a total of 1,562 miles between May $18^{\text {th }} 2012$ and June $18^{\text {th }} 2012$. I personally conducted all thirteen interviews in order to ensure consistency among interviews, as well as to build upon the relationships I had previously established through repeated contact with the participants. Former provider participants were not interviewed in person but instead were interviewed via telephone. I personally conducted all five former provider interviews and took detailed notes of our conversation for subsequent coding.

Next, audio recordings of each current provider interview had to be transcribed. I trained and supervised three undergraduate research assistants to accomplish transcription. This included training regarding transcription conventions and rules, as well as training in the use of Express Scribe software and a transcription foot pedal. Transcription was a time consuming endeavor that took place from May 2012 to 
December 2012. After each transcript was prepared, I carefully reviewed the text to address questions, decipher unintelligible segments, remove identifying information, and correct typos. During this process I took detailed notes about the content of interview transcripts in order to use this information to build upon the pre-existing codebook. I then submitted each transcript back to the participant for review. I incorporated any suggested revisions, modifications, or additions into the transcript.

After the participants approved transcripts, I participated in and monitored the coding process for current and former provider interviews. Subsequent to the transcript review process, I modified the codebook to capture experiences that were not previously incorporated but were useful to address the research questions. Next, I provided detailed training to the two coders in terms of the process of qualitative coding, as well as the nuances of the codebook. Once the coders were adequately trained, coding began. I coded transcripts one and two with the coders in order to ensure consistency among my operationalization of various codes and the coders understanding. After each of these transcripts was coded, I facilitated meetings to review the content coded, address questions, and come to a mutual understanding of the coding system. As the coders moved forward to code the remaining transcripts, I entered all coded material into Atlas.ti for subsequent analysis. As coding continued, I also conducted analyses to check Cohen's kappa and ensure interrater agreement was high. In instances where kappa was lower than desired, I revised the codebook and provided additional feedback to coders in order to improve and maintain consistency. At the conclusion of the coding process, I began 
analyzing the qualitative data to address each of the research questions at hand. I was responsible for all analyses and interpretation of the interview data. 


\section{Chapter 3: Results}

The current study addressed four primary research questions that examine current program functioning as it relates to standards $(R Q 1)$, key program staff members' responses to state standards $(R Q 2)$, the process by which participants implemented the standards (RQ3), and responses to state standards as they relate to program compliance with the standards $(R Q 4)$. In order to speak to these research questions, a number of subquestions and hypotheses have been developed. The following section describes the findings related to each research questions, sub-question, and hypothesis. A synopsis of the main findings related to each question or hypothesis of interest can be found in Table 2.While this section focuses on the reporting of relevant findings, in some places where further interpretation is useful for understanding, discussion of the results and implications have been inserted. The preliminary interpretations presented in this section are further extrapolated upon in the discussion section of this document.

\section{Research Question One}

In order to answer $R Q I$ and determine the extent to which BIP practices are in compliance with state standards, 36 survey items relevant to the requirements set forth by standards were examined. These items assessed topics such as program length, group size, victim contact policies, requirements for program completion, and training of facilitators. Each survey item was dichotomized to indicate compliance or noncompliance with each facet of the standards assessed. In order to be included in the computation of a compliance score, participants had to respond to $75 \%$ of the compliance 
relevant items (i.e., 27 items). Overall compliance with the standards is reviewed, followed by a detailed depiction of compliance with each component of the standards.

Across the 34 programs for which compliance scores could be generated, programs were in compliance with approximately three-quarters of the components of standards assessed $(M=.75, S D=.11)$. Reported program compliance ranged from meeting $53 \%$ to $97 \%$ of the various components of the state standards (see Figure 3). To understand the variation in reported compliance, adherence to each component of the standards is described below (See Table 3).

Program logistics. First, program logistics discussed in the standards were assessed. Program logistics included basic components of program structure and procedures that are explicated in the standards, specifically, group size, use of written policies, record keeping, program length, co-facilitation, and criteria for program completion (see Table 4).

The state standards indicate that BIP groups should have no more than 15 participants (ODOJ, 2009). Programs serve an average of 10 clients per group $(M=9.96$, $S D=7.21$ ). The reported number of clients per group ranged from 0 to 25 , indicating that some programs, such as those that do not have a large BI component or a large client base, do not always serve BI clients, while other programs serve a large number of BI clients per group. The vast majority of programs $(n=32,94 \%)$ were in compliance with this portion of the standards with only two programs $(6 \%)$ having groups that exceeded 15 participants. 
Oregon state standards specify that program policies and procedures regarding victim safety, program completion criteria, transfers between programs, contact with victims, storage of victim information, and confidentiality of victims must be available in writing (ODOJ, 2009). On average, programs required more than four of the six components of standards regarding written policies and procedures $(M=4.31, S D=$ 1.49). Only $26 \%$ of programs $(n=9)$ reported requiring all six written policies and procedures, while $74 \%(n=26)$ required fewer than six. Next, each of the six requirements was examined independently (see Figure 4). In line with this component of the standards, more than three fourths $(n=27,77 \%)$ of programs reported having written victim safety policies. Nearly every program $(n=34,97 \%)$ reported having written criteria for program completion. Less than half of the programs $(n=15,43 \%)$ reported having written policies and procedures regarding client transfers between programs. Seventy-one percent of programs $(n=24)$ reported having written policies and procedures concerning program contact with victims. Less than two-thirds of programs ( $n$ $=21,64 \%$ ) have written policies for storing victim contact information. Most programs $(n=30,88 \%)$ have written policies for ensuring victim/partner confidentiality.

Not only are programs required to have written documentation of some policies and procedures, Oregon state standards also require programs to keep participant records that include each individual's status regarding program completion (ODOJ, 2009). While the standards stipulate that this should occur, only two-thirds $(n=23,66 \%)$ of programs reported keeping a record of how many clients complete the program after intake. 
As of fall 2011, Oregon state standards required a standardized program length of at least 48 weekly sessions (ODOJ, 2009). Nearly every program $(n=32,94 \%)$ reported that a specific number of weekly sessions were required to complete the program. Fewer programs $(n=25,76 \%)$ reported requiring at least 48 weeks of participation. Across all programs, providers indicated that on average 44 weeks $(S D=11.95)$ of intervention are minimally required to complete the program, though this requirement ranged from 1 to 52 weeks. Additionally, on average, participants complete the program after 49 weekly sessions $(S D=8.16)$, though this timeframe ranged from 25 to 60 weeks. This indicates that although some programs may not be in compliance by requiring 48 weekly sessions, on average program participants are receiving slightly more weeks of intervention than the standards mandate.

The use of mixed-gender co-facilitation is advocated in the standards and the standards note that this method of facilitation should be used whenever possible (ODOJ, 2009). Additionally, programs are required to inform their local supervising authority and local domestic violence council if they are not utilizing mixed-gender co-facilitation (ODOJ, 2009). Due to the strong encouragement and supplementary steps that must be taken if co-facilitation is not utilized, this was considered a component of compliance. The majority of programs $(n=27,77 \%)$ offer co-facilitated groups. Of the programs that offer co-facilitated groups, 69\% $(n=18)$ use this method of co-facilitation for all groups in the program. Slightly less than one-third of programs $(31 \%, n=8)$ that utilize cofacilitation did not use mixed-gender co-facilitation for all groups and instead used this method for only some of the groups. Specifically, for programs that only use mixed- 
gender co-facilitation for a portion of groups, this facilitator strategy was utilized for $50 \%$ to $89 \%$ of groups within the programs. When considering all programs, only $56 \%(n=$ 18) reported utilizing mixed-gender co-facilitation for all groups.

Programs are directed to develop written criteria for program completion. In addition to the requirement that this information must be written, the standards also specify discrete requirements for completion that must be fulfilled by participants. These requirements include compliance with program attendance policies, compliance with program rules, compliance with group rules, and the creation of an accountability plan (ODOJ, 2009). Of those who have written completion criteria $(n=34), 88 \%(n=30)$ required each of these four components for completion, while $12 \%(n=4)$ required three of the four components. On average, programs required $3.88(S D=.33)$ completion requirements. Each completion requirement was examined independently in order to determine which completion requirements were most problematic (see Figure 5). Every program $(n=34,100 \%)$ included attendance and compliance with program rules as a necessity for program completion. A small proportion of programs did not require compliance with group rules $(n=2,6 \%)$, or completion of an accountability plan $(n=2$, $6 \%$ ). While two programs did not require the completion of an accountability plan to complete the program, all programs $(n=35,100 \%)$ indicated that batterer accountability is part of the curriculum given to clients at group meetings.

Training of facilitators. After program logistics were considered, training of group facilitators was examined (see Table 5). The state standards require facilitators to have completed 40 hours of victim advocacy training and 40 hours of batterer 
intervention training. These trainings may include a variety of topics including risk factors for perpetration, impact of IPV on children, overview of the criminal justice system, and overview of pertinent laws (ODOJ, 2009). Further, facilitators are also directed to complete at least 32 hours of continuing education every two years. There is some flexibility for interns and facilitators in training in order to provide time to achieve the training requirements. In order to determine compliance with this component of the standards, interns were not considered and instead the proportion of facilitators who had accomplished the 40 hours of victim advocacy and 40 hours of BI training was examined. In total, just over half of the programs $(n=18,56 \%)$ reported that all facilitators had completed victim advocacy training. Similarly, $56 \%$ of programs $(n=18)$ reported that all facilitators had completed the BI training. While just more than half of the programs reported all facilitators had met these requirements, most facilitators within each program had met the victim advocacy training requirement $(n=32,74 \%)$ and the BI training requirement $(n=32,80 \%)$.

Program intervention strategies. While the state standards allow some flexibility in the exact curriculum and philosophy utilized by BIPs across the state, they do stipulate some guidelines to which programs are expected to adhere (see Table 6). First, programs are prohibited from identifying any of the following as a primary cause of battering: poor impulse control, anger, past experience, unconscious motivations, substance use or abuse, low self-esteem, and client or victim's mental health problems (ODOJ, 2009). Most programs $(n=28,80 \%)$ did not endorse any of these as a primary cause of battering and thus were in compliance with this component of the standards. 
While this is the case, one-fifth $(n=7)$ did identify at least one of these areas as a primary cause of battering. Next, the seven programs that endorsed at least one of the noncompliant primary causes of battering areas were examined further (see Figure 6). Of these programs, the majority $(n=4,57 \%)$ endorsed three or fewer of these as primary causes of battering, while three programs (43\%) endorsed four or more as primary causes of battering $(M=3.71, S D=2.36)$. The primary causes of battering selected by these programs included past experience $(n=5)$, low self-esteem $(n=5)$, poor impulse control $(n=4)$, unconscious motivation $(n=4)$, anger $(n=3)$, client or victim's mental health problems $(n=3)$, and substance use or abuse $(n=2)$.

In addition to stipulations as to what programs may not identify as a primary cause of battering, the standards also indicate what features should not be included in program curriculum. Specifically, the intervention strategies cannot view battering as an addiction and the victim as enabling or co-dependent; encourage ventilation techniques such as punching pillows or other expressions of rage; blame the client's decision to batter on the victim's qualities or behavior; use actions or attitudes of moral superiority or controlling or abusive behaviors toward clients; require victim or partner disclosure of information or participation; encourage victim or partner disclosure of information or participation; support, recommend, or utilize couples, marriage, or family counseling, or medication as intervention; and view battering as a bi-directional process with responsibility shared by the victim (ODOJ, 2009). Most programs $(n=29,83 \%)$ did not endorse any of these strategies. Of the six programs (17\%) that endorsed at least one prohibited intervention strategy, four programs (67\%) only endorsed one prohibited 
strategy, and two programs (33\%) endorsed two prohibited strategies. The prohibited strategies were examined in more detail in order to determine which specific strategies were selected by programs (see Figure 7). Most frequently programs indicated that they encourage victim or partner disclosure of information or participation $(n=3)$, or they support, recommend, or utilize couples, marriage, or family counseling, or medication as an intervention for battering $(n=3)$. Only one program indicated they require victim or partner disclosure of information or participation. Additionally, one program indicated the use of actions or attitudes of moral superiority or controlling or abusive behaviors towards clients. All other prohibited intervention strategies were not endorsed by any program.

While some of the regulations in the standards include intervention strategies that are not permitted, the standards also contain intervention strategies that must be included. Intervention strategies must include the following: attempt to increase clients' understanding of the causes, types, and effects of their battering behavior; use of respectful confrontation; address tactics used to justify battering; increase client recognition of the criminal aspect of his thoughts and behavior; reinforce client identification and acceptance of personal responsibility and accountability; reinforce "appropriate" respectful beliefs and behavioral alternatives; promote client recognition of and accountability for patterns of controlling and abusive behaviors and their impacts; ensure that the impact of battering on victims, partners, and children remains in the forefront of intervention work (ODOJ, 2009). The majority of programs $(n=28,80 \%)$ included all of these listed intervention strategies. Among the few programs $(n=7,20 \%)$ 
that did not use all required intervention strategies, almost all of the programs used almost all of the strategies. Specifically, all of these programs utilized at least six of the eight intervention strategies listed by the standards and $86 \%(n=6)$ utilized at least seven of the eight intervention strategies (see Figure 8). Each component of approved curriculum was examined for programs that did not endorse all eight portions of the curriculum requirements. Most frequently, programs indicated they do not increase client recognition of the criminal aspect of his thoughts and behavior $(n=5,71 \%)$.

Additionally, one program (5\%) did not endorse each of the following curriculum requirements: curriculum increases clients' understanding of the causes, types, and effects of battering behavior; curriculum reinforces client identification and acceptance of personal responsibility and accountability for the use of abusive tactics; and curriculum reinforces appropriate respectful beliefs and behavioral alternatives. All programs endorsed all other approved curriculum components. An additional component of program curriculum that was not included in the compliance computation but is important to note is the use of culturally appropriate curriculum. The standards indicate that culturally appropriate curriculum and intervention should be utilized whenever possible but do not mandate that all programs incorporate this approach. Though this approach is encouraged, less than half of programs $(n=15,43 \%)$ indicated use of a culturally specific curriculum.

Policies relating to victims and partners. The standards emphasis on victim safety is introduced in the purpose of the standards and this focus is apparent throughout the standards. References to policies relating to victims and partners are woven 
throughout various areas of the standards (see Table 7). First, the standards require that victim or partner information should only be available to BIP staff with a specific need for the information and should not be disclosed without the authorization of the victim or partner (ODOJ, 2009). Participants were asked to indicate which individuals had access to victim or partner information and most programs $(n=27,79 \%)$ indicated only designated staff members (e.g., program director and group facilitators). Seven programs $(21 \%)$ indicated that representatives from nonprofit victim advocacy programs had access to victim contact information. It is important to note that while the standards indicate this should not be the policy universally, it is acceptable with victim or partner authorization.

In addition to requirements for access to victim contact information, the standards also stipulate when victim or partner contact is "appropriate". According to the standards, contact with victims or partners is only acceptable in specific circumstances. These circumstances include: to notify them as to whether the client was accepted into the program; to tell them about the client's attendance record; to tell them the client has been discharged or terminated; to tell them general information about the program; to inform them of immediate or imminent threat; and to provide information about community resources. Participants were asked in what circumstances victims or partners are contacted and provided a list of acceptable circumstances for contact, as well as two unacceptable circumstances for contact. Specifically, the unacceptable circumstances included contact to tell them about things the client said in the group and to solicit information about the client from the victim or partner. Of the 29 programs (85\%) that indicated ever having contact with a victim or partner, more than two-thirds $(n=20$, 
$69 \%$ ) only contact victims or partners in "appropriate" circumstances. Nine programs (31\%) reported contacting victims or partners in a prohibited circumstance. All of these programs $(100 \%)$ indicated contact with victims or partners to solicit information about how a client is doing in the home and one program (11\%) also indicated contacting victims to tell them about things the client has said about them during group meetings.

The standards indicate that programs can contact victims and partners in order to provide information about the program and community resources. This can be done through the program or in collaboration with a victim advocacy agency (ODOJ, 2009). While this is the case, slightly less than three-fourths of programs $(n=25,74 \%)$ indicated they distribute informational materials to victims and partners. In addition to encouraging programs to provide informational materials to victims and partners, the standards indicate what the distributed materials should include. Specifically, materials should include victim advocacy resources, information about victims' rights (e.g., informing the victim they are not required to participate, informing the victim about what information they can obtain from the program, etc.), information for safety planning, a description of the BIP, a statement about the limitations of BIP outcomes, and information about contacting or being contacted by the program (ODOJ, 2009). Of the 25 programs that specified they distribute information to victims or partners, only 10 programs (40\%) indicated the materials include all types of information specified in the standards. Slightly more than half of these participants $(n=8,53 \%)$ included at least four of the six types of information, while the remaining programs $(n=7,47 \%)$ included three or fewer of the types of information in their victim/partner information. Most commonly programs did 
not include information about safety planning $(n=9,36 \%)$. Other information that programs did not distribute included information about victims' rights $(n=8,32 \%)$, limitations of BIP outcomes $(n=7,28 \%)$, program description $(n=6,24 \%)$, contacting or being contacted by the program $(n=6,24 \%)$, and victim advocacy resources $(n=1$, 4\%) (see Figure 9). Finally, the standards also indicate that when requested, the materials should be made available in languages other than English (ODOJ, 2009). Despite this portion of the standards, only approximately one-third of programs $(n=9,36 \%)$ reported having these materials available in languages other than English.

Community collaboration. In addition to placing victim safety at the forefront of the standards, another area that is inherent in the purpose of the standards is that of community collaboration. The purpose of this section of the standards encourages communication and interaction in the community to ensure a community wide effort to stop IPV (ODOJ, 2009). Given this prominence in the purpose, it is not surprising that the standards include multiple provisions relating to community collaboration (see Table 8). Victim advocacy agencies are one collaborative partner emphasized in the standards as an important collaborative partner (see Figure 10). First, the standards require that each program must have contact with a victims' advocacy program, including naming a designated liaison for collaboration (ODOJ, 2009). Most programs $(n=32,91 \%)$ indicated having contact with a victims advocacy program and of those programs, $91 \%$ ( $n$ $=29$ ) reported having a staff member from the program designated to act as a liaison to the victim advocacy agency. In addition to this relationship, the standards also indicate that BIPs should submit their policies and procedures to a victim advocacy agency for 
review (ODOJ, 2009). Of the 32 programs that have contact with a victim advocacy agency, slightly more than two-thirds $(n=22,69 \%)$ reported having a victim advocacy agency review the program policies, procedures, and materials. Programs are also expected to participate in their local DV council if one exists (ODOJ, 2009). The majority of programs $(n=30,88 \%)$ indicated they are located in a county or region that has a local DV council, while three programs (9\%) indicated one did not exist and one program (3\%) indicated they were unsure. Each of the four programs that responded that a DV council does not exist or they are not sure is located in a rural area. Of the programs that indicated a DV council does exist in their area, $87 \%(n=26)$ reported that a member of their staff attends meetings held by the council. Four programs (13\%) indicated that a staff member does not attend the DV council meetings despite a council functioning in their area.

While collaboration with victim advocacy agencies and DV councils is stressed in the standards, they also outline the necessity for collaboration with the criminal justice system (see Figure 11). Specifically, BIPs are expected to be in contact with the local supervisory authority or mandating authority, including having a liaison to communicate with this body (ODOJ, 2009). Nearly three-fourths of programs $(n=25,71 \%)$ reported collaboration with the local supervisory authority or mandating authority. When asked broadly whether the program had a liaison to the criminal justice system, nearly every program $(n=32,94 \%)$ reported such a liaison exists. Through this collaboration the standards indicate that programs should provide information about participation in the program, such as program outcomes and attendance. Seventy percent of programs $(n=$ 
23 ) indicated they communicate both program outcome and attendance information, $24 \%$ of programs $(n=8)$ indicated communicating either program outcome or attendance information, and $6 \%$ of programs $(n=2)$ indicated communicating neither program outcome or attendance information. Of the 10 programs that did not provide both types of information, all indicated that they did not communicate about program outcomes and two indicated they did not communicate regarding attendance.

The standards also mandate collaboration outside of victim advocacy programs and criminal justice programs. Specifically, the standards indicate that programs should collaborate with other BIPs and participate in larger local or statewide BIP organizations (ODOJ, 2009). Most programs $(n=29,83 \%)$ report collaboration with other BIPs, but less than half $(n=15,43 \%)$ belong to a broader BIP organization. Finally, the standards require programs, to the extent practically possible, to participate in trainings in the community in order to educate about and raise awareness for the issue of IPV (ODOJ, 2009). Less than half ( $n=15,43 \%)$ of the programs reported adhering to this component of the standards by assisting in the trainings for others in the community.

High and low compliance. In order to proceed with the study and identify experiences of implementation for those with varying levels of compliance, the top $20 \%$ and bottom $20 \%$ of programs were selected based on compliance. Initially 15 programs were identified as potential high or low compliance programs. Seven programs were considered high compliance with compliance scores ranging from .86 to .97 . On average high compliance programs met $91 \%$ of the assessed components $(M=.91, S D=.05)$. Due to a tie in compliance score, eight programs were initially considered low 
compliance with compliance scores ranging from .53 to .64. On average low compliance programs met $60 \%$ of the assessed components $(M=.60, S D=.04)$. While these programs were initially selected and all high compliance programs agreed to participate, two low compliance programs declined to participate in Phase Two of the study. This resulted in a final sample of seven high compliance programs and six low compliance programs as participants in Phase Two of the study. The final low compliance participants had compliance scores ranging from .53 to .64 . On average these programs complied with $59 \%$ of the assessed components $(M=.59, S D=.04)$.

\section{Research Question 2}

After establishing the extent to which BIPs in Oregon are in compliance with standards, $R Q 2$ was assessed. This research question asked how key BIP representatives in Oregon have responded to the state standards. In order to address this question, seven sub-questions and three hypotheses were evaluated utilizing interview data obtained through intensive semi-structured interviews with key program staff. In order to interpret the findings below it is important to consider the date when each program began providing BIP services. All of the programs that provided this date $(n=10)$ began providing BIP services prior to the formal adoption of the standards (range $=1983$ to 2006). This information provides context when examining findings related to actual control, perceived control, attitude change (including the possible explanations of rationalization and reactance), legitimacy, and implementation. Specifically, each of these programs had practices and procedures in place prior to the formal introduction of the standards. Thus, they likely did not initially develop their program policies and 
characteristics in light of the standards, but rather adapted their program procedures to align with the standards after their introduction.

Actual control. First, $R Q 2 a$ was evaluated to determine the extent to which key program representatives reported experiences of actual control over the content and development of the state standards. Experiences of actual control were assessed with two codes: high actual control and low actual control (see Appendix F Section 1). The extent to which participants reported experiences consistent with actual control varied. The average actual control ratio was $.46(S D=.52$, ranging from $0.00-1.00)$, indicating that across all programs, $46 \%$ of experiences described by participants related to actual control were indicative of high actual control (see Table 9). Slightly less than half of the programs $(n=6)$ reported more experiences of high actual control as compared to low actual control (actual control ratios ranging from .60 to 1.00). A majority of programs (n $=7$ ) reported more experiences of low actual control as compared to high actual control (actual control ratios ranging from 0 to .43). Thus, while participants reported a variety of different experiences related to actual control, they tended to report more experiences consistent with low actual control as opposed to high actual control.

When the content of responses was assessed (see Appendix F Section 1) several important findings came to light. Only three participants actively served on the original Governor's Committee or the Standards Advisory Committee. Further, only two additional participants described opportunities to participate on the Standards Advisory Committee or to provide input to the Governor's Committee or Standards Advisory Committee regarding the standards. These five individuals accounted for 32 of the 46 
(70\%) instances of high actual control. Beyond these individuals, few participants were able to outline the process by which the standards were created. While this was the case, the majority of participants were aware of at least one key individual related to the standards (e.g., name of a member of the Standards Advisory Committee). The eight participants who were not on either committee nor had the opportunity to contribute to the committees accounted for 26 of the $30(87 \%)$ instances of low actual control. These participants were not only inactive with regards to the Governor's Committee and Standards Advisory Committee, but also had very limited knowledge about the standards creation process. This indicates that knowledge regarding the standards creation process appears to be primarily limited to those who were involved in the Governor's Committee or Standards Advisory Committee or who had an opportunity for involvement. Thus, those who were not involved in some capacity were not able to achieve high levels of actual control through other means, such as awareness of the process. For these participants, control over the standards appears to be limited to knowledge of key individuals related to the standards.

Perceived control. Next, $R Q 2 b$ was assessed to determine the extent to which participants experienced perceived control over the content and development of the standards. This construct was assessed with four codes; high perceived ability; high procedural justice; low perceived ability; and low procedural justice (see Appendix F Section 2). The extent to which participants reported experiences consistent with perceived control varied (see Table 9). The average perceived control ratio was $.34(S D=$ .29 ; ranging from $0.00-1.00$ ), indicating that across all programs, $34 \%$ of experiences 
described by participants related to perceived control were indicative of high perceived control. The majority of programs $(n=9)$ reported a higher frequency of low perceived control perceptions as compared to high perceived control perceptions (perceived control ratios ranging from $0-.43$ ). Two participants reported a greater frequency of high perceived control perceptions as compared to low perceived control perceptions (perceived control ratios ranging from .60 - 1.00). Further, two participants reported an equal frequency of high and low perceived control perceptions. Thus, perceptions of perceived control varied across participants but the majority of participants reported fewer perceptions of high perceived control than perceptions of low perceived control over the standards.

While participants reported a variety of different experiences related to perceived control (see Appendix F Section 2), they tended to report more experiences consistent with low perceived control as opposed to high perceived control. Eight participants described having at least some confidence that they would be able to impact the standards if they desired and those opinions would be taken seriously. Three of these eight (38\%) participants are individuals that have served or currently serve on the Governor's Committee or Standards Advisory Committee, thus increasing the ease at which participation could be achieved. Further, three of the five (60\%) participants who endorsed fairness of the standards process and/or representativeness of the committee have served or currently serve on the Standards Advisory Committee. Their close involvement likely impacts their views of fairness and representation. While most participants did report at least one experience consistent with high perceived control, 
comments related to low perceived control were much more common. When asked whether participation in the further refinement of standards would be possible and whether their input would be valued, participants described an inability to participate or perceptions that their input would not be valued for two primary reasons. First, participants described previous negative interactions with those involved in the community collaborative response to IPV (e.g., the LSA or victim advocacy agencies). In most cases these experiences were not related to the standards or the Standards Advisory Committee but these negative experiences in the IPV community have contributed to a culture in which some providers do not feel they have the opportunity to voice their opinions or that others, such as other BIP providers or victim advocates, would actually value those opinions. Second, participants indicated that their lack of awareness regarding the standards process or those involved in their creation makes them unsure if and how their concerns could be voiced or if those concerns would be valued.

Additionally, most participants made at least one comment questioning the representativeness of the Standards Advisory Committee. Concerns surrounded multiple aspects including lack of representation regarding race/ethnicity, sexual orientation, geographic location, and other cultural contexts. These participants believed that the lack of diversity has impacted the content of the standards making them more appropriate for programs in urban locations or those serving clients from the majority cultures and less appropriate for programs in rural locations or those serving clients from minority cultures. The prevalence of participants who voiced concerns regarding the 
representativeness of the Standards Advisory Committee highlights a substantial gap in the current composition of the Committee and the needs perceived by providers.

A final critique of the Standards Advisory Committee surrounded the power allotted to different sectors of the community collaborative response to IPV, which indicated reduced perceived control. Some participants believed that more diverse stakeholders, especially those from judicial and law enforcement agencies, should have been better represented. Further, concerns were raised regarding the powerful role of community corrections in developing the standards. Some participants indicated that they perceive those from community corrections as misinformed or uninformed about the functioning of BIPs, or skeptical about the outcomes for those who participate in BIPs. These participants fear that the power given to individuals who may not be fully aware of or supportive of the purpose of BIPs may result in standards that are more aligned with supporting the criminal justice system rather than encouraging change for participants. Overall, the experience of low perceived control appears to outweigh the experience of high perceived control for most participants.

Actual control and perceived control. Understanding experiences of actual and perceived control was necessary to evaluate the first hypothesis. Hypothesis $2 a$ asserts that program representatives who primarily report having actual control over the standards will describe higher perceived control as compared to those who primarily report not having actual control over the standards. In order to examine this hypothesis, participants were split into two groups. This resulted in a high actual control group comprised of six participants and a low actual control group comprised of seven 
participants. The first group included those who reported a greater proportion of high actual control experiences as compared to low actual control experiences. These participants each had an actual control ratio higher than $.50(M=.84, S D=.19 ;$ ranging from $.60-1.00)$. This indicates that $84 \%$ of their comments related to actual control were consistent with high actual control. The second group included those who reported a greater proportion of low actual control experiences as compared to high actual control experiences. Each of these participants had an actual control ratio lower than $.50(M=$ $.24, S D=.18$; ranging from in $0.00-.43$ ). This indicates that $24 \%$ of their comments related to actual control reflected high actual control. Next, experiences of high perceived control and low perceived control were examined for each of the two groups. On average, high actual control participants had a perceived control ratio of $.39(S D=$ .21 ; ranging from $0-.60$ ), indicating $39 \%$ of their comments related to perceived control were indicative of high perceived control. Among the low actual control group, participants had a perceived control ratio of $.30(S D=.36$; ranging from $0.00-1.00)$, indicating that $30 \%$ of their comments related to perceived control related to high perceived control. Thus, descriptively, the high actual control group has an average perceived control ratio $23 \%$ higher than the low actual control group. Though extremely underpowered, an independent samples t-test was conducted to determine if a statistically significant difference in perceived control ratios for the high and low actual control groups could be identified. A significant difference was not detected, $t(11)=-.53, p=$ .61. Thus, while a moderate descriptive difference in perceived control ratios was observed across the two groups, this finding is not statistically reliable. 
In addition, further analyses were conducted that demonstrate that perceived control and actual control are not directly related to each other. First, the experiences of one participant in the high actual control group were examined because while this participant fell in the high actual control group (actual control ratio $=.66$ ), this participant did not describe any perceptions consistent with high perceived control and thus had a perceived control ratio of 0 . The content of this participant's experience was evaluated to better understand why experiences of actual control did not correspond to experiences of perceived control. When examining this individual's experience overall, it is evident that despite awareness of the standards creation process and input regarding the creation of standards, disagreement with some components of the standards has resulted in isolation from the BIP community. As the participant described, "The whole idea of it being community and it being supported is only there at a very surface level...it has become very much an [institutionalized system] and when things become institutionalized we are out to protect the institution and not necessarily to help the people". This observance of a perceived shift in the field of IPV intervention impacted this participant's confidence that they could voice their concerns, be heard, and impact standards in the future. Conversely, one participant who did not report any experiences consistent with high actual control (actual control ratio $=0.00)$ reported $100 \%$ of his/her beliefs regarding perceived control consistent with high perceived control. This participant was adamant that they could accomplish anything they sought to accomplish, regardless of prior involvement with the standards. For instance, this participant indicated, "I think I could talk to the right people and... go to meetings and bring [my concerns] up". Even more broadly this participant 
claimed, "I think I could do anything". These findings indicate how perceived control and actual control can be unrelated. Actual control over the standards is likely only one factor in determining the extent to which perceived control occurs. Other factors, such as confidence in one's own abilities generally, or previous negative experiences, may play a crucial role in understanding whether perceived control is developed.

Negative attitude change and maintenance. The extent to which those with initial negative attitudes towards the standards shifted or maintained their attitudes was assessed next, to address $R Q 2 c$ and $R Q 2 d$. Research Question $2 c$ asks whether participants described experiences consistent with the phenomenon of rationalization. Research Question $2 d$ asks whether participants described experiences consistent with the phenomenon of reactance. These phenomena were assessed through the use of four codes; positive initial response, negative initial response, positive current response, and negative current response(see Appendix F Section 3; see Table 9). In the current study, experiences in which the participant initially viewed the standards as negative and currently view the standards as positive served as a proxy for rationalization as it may be one potential explanation for the shift in attitudes. Experiences in which the participant initially viewed the standards as negative and currently view the standards as negative served as a proxy for reactance as it may be one potential explanation for the maintenance of negative attitudes. Thus, in order to examine attitude change, and by extension the possible experiences of reactance and rationalization, only participants who experienced a primarily negative initial response to the standards were examined. This includes the eight participants who endorsed an equal or greater number of experiences 
consistent with negative initial response as compared to positive initial response. In other words, these eight individuals had initial response ratios of .50 or lower, indicating $50 \%$ or less of their comments related to initial response where characterized as positive initial response. The ratio of current responses was evaluated for each of these eight participants. This information allowed for the determination of whether those with a primarily negative initial response towards the standards have maintained their negative views (i.e., a proxy for reactance), or if they have modified their views to become more positive (i.e., a proxy for rationalization).

Of the eight participants with a primarily negative initial response, four (50\%) maintained a primarily negative view of the standards, indicating reactance could possibly have occurred. Specifically, these four participants received a greater number of negative current response codes as compared to positive current response codes. This resulted in current a response ratio below .50 for each of these four participants $(M=.32$, $S D=.17$; ranging from $.06-.43$ ). This indicates that only $32 \%$ of their comments related to current response were reflective of a positive current response. The remaining four participants $(50 \%)$ had a primarily positive current response to the standards, indicating rationalization could have possibly occurred. Specifically, these four participants received a greater number of positive current response codes as compared to negative current response codes. This resulted in current a response ratio above .50 for each of these four participants $(M=.59, S D=.06$; ranging from $.55-.67)$. This indicates that $59 \%$ of their comments related to current response were reflective of a positive current response. Thus, of the eight participants who initially viewed the standards as primarily 
negative, $50 \%$ changed to view the standards as primarily positive (i.e., a proxy for rationalization) while $50 \%$ maintained their negative attitudes (i.e., a proxy for reactance). Next, the content of experiences was assessed for those who changed and maintained their initial negative attitudes towards the standards.

First, responses from the four participants who changed their initial negative attitudes towards the standards to be primarily positive (i.e., a proxy for rationalization) were examined in more detail. All four of these participants indicated that when they first learned about the standards they experienced great fear regarding the content and enforcement of the standards. One participant remembered when they first learned of the standards, "I almost threw up". Another described anxiety relating to fear of consequences for non-compliance, "[I thought] what the hell is this? Am I going to get in trouble because I'm not doing something?". Another simply stated, "I was scared". These participants also noted more specific worries including the lack of standards for other community partners. One participant explained, "When you put in rules that focus on one specific area of domestic violence, especially relating to batterers, then the victims' voices are lost. It takes the focus even further off a coordinated community response". Another participant explained that the lack of clarity regarding the local supervisory authority caused initial anxiety, "I understood the intent and I think in theory it can be good, the problem [is designating the local supervisory authority], when you put on an agency who has a different goal than we do, that's where the huge concern is". Three of the four participants explicitly described the process by which they became more accepting of the standards as they learned more about them over time, while the 
remaining participant did not discuss the process explicitly. One participant clearly explained the shift as they perceived it, "I think it's just a little bit more familiar... I think there's a clearer understanding of what the expectations are from everybody involved. So, I think that's eased the tension quite a bit... becoming more familiar and more aware". Another provider began to feel more at ease with the standards after thoroughly reviewing their content, "I actually felt a little bit better when I really read them over because it didn't feel ... that there was someone trying to tell me how to provide services when I know I'm the expert". The third participant indicated that over time the standards have become a positive aspect of their program, "I've come to appreciate them more". The final participant did not describe a shift but instead appeared to simply accept that standards are now a reality, "If they have to be there and there's gonna be standards... they're okay, we would be just fine". Interestingly, two of the participants who reported changing their initial negative attitudes towards the standards to be primarily positive are located in an area where local standards, which are different in some ways from the state standards, are enforced rather than state standards. Thus, while these participants currently view the state standards positively, these standards are not the ones they are expected to adhere to for local referrals.

Next, the four participants who maintained their initial negative attitudes towards the standards (i.e., a proxy for reactance) were examined in more detail. Similar to what was observed among those who changed their negative attitudes, these providers had initial concerns. While this is the case, the content of these concerns was more focused on specific components with which they disagreed as opposed to overarching fear or 
confusion. Initially, three of these participants reported having substantial concerns with the standards, including the exclusive focus on male offenders, the lack of flexibility, the one-size-fits-all approach, and the prematurity of the standards given what was known in the field. One participant explained the conversations they had early on when the standards were first being discussed, "We were trying to say [that] this is not the time. We don't know enough to have standards. We don't know what works and what doesn't. The research is still in its infancy". Another participant focused their initial negative perceptions on the extent to which their program would have to change and the feeling that the change required would be too difficult to accomplish, "I'm willing to do them but some of them seemed unachievable. Two weeks of training is a lot of time away from work... I understand why we need training but that's two whole work weeks". These participants differed from those that reported attitude change because they did not report a change from their similar initial reactions in perception, appreciation, or endorsement of the standards. Instead, these participants focused on components of the standards with which they remain in disagreement. Most comments were directed at specific components or characteristics of the standards. All four participants voiced issues related to program length, with some participants wanting more flexibility to assign longer or shorter lengths to individuals and others wanting programs to require more than 48 weekly sessions. Three participants disagreed with the standards' lack of individualized treatment, focus on male batterers only, necessity of male-female co-facilitation, and restrictions from providing couples or family counseling. Two participants disagreed with the requirement for 40 hours of victim advocacy training. Additionally, references to the 
rigidity of the standards and the lack of room for clinical judgment were raised. For instance, "One thing I would personally like to see is [wording] that gives the counselor or facilitator more room for clinical judgment, rather than this blanket way it's got to be done". Participants also voiced their concern over the state of the standards and the role that BIPs play in IPV intervention, "From my standpoint the standards have become more punitive than helpful. [They are] not helpful or therapeutic". The discrepancies between the standards and these participants' view of ideal practice fueled the feeling that the standards could be better than they are currently. As one participant described, "I honestly and truly believe that we can do a whole lot better job than we're doing".

These analyses of participants' change or maintenance of negative attitudes towards the standards can be summarized in two ways. While, this paragraph provides some interpretation of the results related to attitude change and maintenance, these constructs will be more fully reviewed within the discussion section. First, as operationalized, change and maintenance of negative attitudes were both experienced in this sample of BIP representatives. Further, these processes were experienced to an equal extent with approximately $31 \%$ of the sample describing a series of experiences consistent with attitude change (i.e., a proxy for rationalization) and $31 \%$ of the sample describing a series of experiences consistent with attitude maintenance (i.e., a proxy for reactance). This indicates that despite all eight of these programs initially disagreeing with the standards, some participants changed their perspective about the standards over time, while others maintained their negative perceptions. Initial response ratios were examined for the attitude change and attitude maintenance participants to determine if 
perhaps the attitude maintenance participants had more a more negative initial response to the standards. This did not appear to be the case, with an average initial response ratio of $.33(S D=.15)$ for attitude change participants and an average initial response ratio of $.32(S D=.22)$ for attitude maintenance participants. Thus, it appears that attitude change and attitude maintenance in this context do not depend on the gravity of initial negative perceptions. Second, it is important to note that while attitude change and attitude maintenance emerged from similar ratios of initial negative and positive responses, the experiences comprising attitude change and attitude maintenance participants were quite unique. Those who reported changing their initial negative attitudes towards the standards tended to highlight how their views evolved over time and the process by which they came to accept, and in some cases appreciate, the standards. These participants described that education and familiarity allowed them to become better acquainted with the standards and view the standards more positively. Conversely, those who reported maintaining their initial negative attitudes were much more focused on the components of the standards with which they continue to disagree. Thus, it appears that the ability to educate themselves and accept the standards as part of the BIP culture allowed participants to shift their negative views. This suggests an interesting intervention opportunity. It is possible that if the participants who report maintenance of negative attitudes were to be exposed further to the nuances of the standards and have the ability to learn more about them, they may perceive them in a more positive light. In addition to gaining an understanding of whether attitude change and attitude maintenance experiences exist for key BIP representatives, as well as the quality of those experiences, 
an additional factor, absoluteness, theorized to impact whether attitude change or maintenance occur was assessed. See the discussion section of the current document for a more thorough review of the implications of these findings.

Absoluteness. After establishing the extent to which negative attitude change and maintenance occurred among participants, $H 2 b$ was evaluated. This hypothesis predicted that program representatives who respond to the standards with rationalization (i.e., retrospective report of changing initial negative attitudes) view the standards as more absolute than program representatives who respond to the standards with reactance (i.e., retrospective report of maintaining initial negative attitudes). Experiences of absoluteness were assessed with two codes; absoluteness and non-absoluteness (see Appendix F Section 4; see Table 9). The average absoluteness ratio was $.58(S D=.33)$, indicating that on average $58 \%$ of comments related to absoluteness were related to the presence of absoluteness rather than it's absence (non-absoluteness). Eight participants (62\%) reported a higher frequency of absoluteness perceptions as compared to non-absoluteness perceptions. One program (8\%) reported an equal number of absoluteness and nonabsoluteness perceptions. The remaining participants $(n=4 ; 31 \%)$ reported a higher frequency of non-absoluteness perceptions as compared to absoluteness perceptions. Thus, most participants reported primarily absolute perceptions towards the standards. After establishing the extent to which participants changed and maintained their initial negative attitudes, as well as whether or not participants viewed the standards as absolute, $H 2 b$ was evaluated. The average absoluteness ratio for negative attitude change (i.e., a proxy for rationalization) participants was $.58(S D=.25$; ranging from .22 to .80$)$. 
This indicates that on average, $58 \%$ of these participants' comments related to whether the standards were absolute were indicative of absoluteness. Thus, when the negative attitude change participants are examined as a group, their view of the standards as primarily absolute is consistent with $H 2 b$. While aggregated responses are valuable, individual absoluteness ratios were also evaluated. Three of these four negative attitude change participants viewed the standards as primarily absolute, indicated by an absoluteness ratio over .50 . This indicates that $75 \%$ of the participants who changed their initial negative attitudes towards the standards to be primarily positive also made more comments indicative of absoluteness as opposed to non-absoluteness. One participant (25\%) reported changing their initial negative attitudes but viewed the standards as nonabsolute, with an absoluteness ratio of .22. This participant does not follow the pattern explicated in $H 2 b$.

Next, perceptions of absoluteness were evaluated for participants who maintained their initial negative attitudes towards the standards (i.e., a proxy for reactance). The four participants who maintained negative attitudes were evaluated to determine the extent to which they viewed the standards as absolute. The average absoluteness ratio for these participants was $.71(S D=.39$, ranging from .17 to 1.00$)$, indicating $71 \%$ of the comments related to the extent to which standards are absolute were indicative of absoluteness as opposed to non-absoluteness. This finding is contrary to what was expected. When individual absoluteness ratios were examined, this pattern continued. Specifically, three of the four participants $(75 \%)$ discussed the standards as primarily absolute as opposed to non-absolute, as indicated by an absoluteness ratio over .50. One 
participant $(25 \%)$ viewed the standards as primarily non-absolute with an absoluteness ratio of $17 \%$. Thus, it appears that most participants included in this analysis viewed the standards as absolute, regardless of whether they changed or maintained their initial negative attitudes towards the standards. Further, counter to expectations, those that changed their initial negative attitudes actually had lower absoluteness ratios on average $(M=.58)$, compared to those that maintained their initial negative attitudes $(M=.71)$. Though extremely underpowered, an independent samples t-test was conducted to determine if a statistically significant difference in absoluteness ratios for the negative attitude change and maintenance groups could be identified. A significant difference was not detected, $t(6)=-.55, p=.60$. Thus, this finding is not statistically reliable. The content of the interview discussion coded as absoluteness and non-absoluteness was examined for the negative attitude change and maintenance groups to determine if differences in the content of comments existed. This examination did not yield any systematic or noticeable differences across the two groups. Their comments tended to be similar and the content did not differentiate the two groups. Thus, $H 2 b$ was not supported in the current study.

Legitimacy. Next, $R Q 2 f$, which aims to understand the extent to which participants view the standards and the standards creation process as legitimate, was examined. The current study operationalized high legitimacy as perceptions of procedural justice, positive norms, and policy logic. Alternatively, low legitimacy was operationalized as a lack of perceptions of procedural justice, negative norms, and the lack of policy logic. In order to create a measure of legitimacy, the sum of instances of 
high procedural justice, positive norms, and high policy logic were divided by the sum of high and low procedural justice, positive and negative norms, and high and low policy logic (see Appendix F Section 5; see Table 9). This process produced a ratio that indicates the extent to which participants perceived the standards as legitimate, with zero indicating perceptions consistent with the absence of legitimacy exclusively, and 1.00 indicating perceptions consistent with the presence of legitimacy exclusively. Across participants, the average legitimacy ratio was $.40(S D=.24$, ranging from $.07-.86)$. This indicates that $40 \%$ of comments related to legitimacy were related to the presence of perceived legitimacy rather than it's absence. Four participants $(31 \%)$ reported a higher frequency of experiences and perceptions indicative of legitimacy as compared to experiences and perceptions indicative of a lack of legitimacy. It is important to note that of these four participants, two did not report any experiences consistent with high or low procedural justice and positive or negative norms. Thus, their legitimacy ratios are based solely on their reports of high and low policy logic. This limits the extent to which their perceptions of legitimacy can be examined as they did not discuss two components of the operationalization of this construct. One participant reported an equal number of experiences consistent and inconsistent with legitimacy. The remaining participants $(n=$ 8) reported a higher frequency of experiences and perceptions inconsistent with legitimacy. Again, one participant in this group did not report any experiences consistent with high or low procedural justice and positive or negative norms. Because of this, their legitimacy ratio is based solely on perceptions of high and low policy logic. 
Together, these findings provide information that addresses $R Q 2 f$. Specifically, when the components of legitimacy are examined as a whole it appears that most providers viewed the standards and their creation process as lacking in legitimacy to a greater extent than they viewed the standards and their creation process as legitimate. This pattern held across all three components of legitimacy. Specifically, on average participants' procedural justice ratios, norms ratios, and policy logic ratios all indicated that participants tended to have views of the standards inconsistent with aspects of legitimacy.

When the content of these experiences and perceptions was examined, several important findings were realized. First, while some participants indicated the standards creation process was fair and the Standards Advisory Committee represents most providers, the majority of participants questioned the representativeness of the Standards Advisory Committee. Specifically, participants noted that diversity in many areas (e.g., racial/ethnic diversity, geographic diversity, sexual orientation diversity) was lacking. These concerns played a primary role in participants' view of the standards as primarily low in procedural justice. Second, many participants did not provide insight into the norms surrounding standards in the community. While most participants did report participating in discussion about the standards in the community, many indicated these discussions were not about whether the standards were viewed favorably but rather about learning the specifics of what the standards entail. While this was the case, for those who did describe norms in the community, the negative norms code was employed to a greater extent than the positive norms code. This highlights that those who have perceived 
community norms related to standards primarily view those norms as negative. Third, while most participants viewed the standards as being based in best practice, most did not believe the standards were truly evidence-based. Importantly, almost every participant who discussed the lack of evidence-based standards noted that this was not due to oversight within the Standards Advisory Committee, but instead was a reflection of the state of the field. In sum, each component of legitimacy was primarily experienced as lacking across participants. Thus, on average overall legitimacy was only observed in $40 \%$ of participants. This indicates that participants tended to view the standards and their creation process as lacking legitimacy.

Relation of Standards to Program Closure. While determining how current BIP providers have responded to the state standards is necessary, identifying the extent to those who once provided services but have since stopped is also imperative in order to determine whether standards have impacted the survival of programs over time. The inclusion of former providers allows for a deeper understanding of how standards may or may not have played a role in programs' decisions to stop providing BIP services. In order to address this, $R Q 2 g$ asks how state standards have impacted BIP closures across the state of Oregon among a sample of five former providers. This research question was addressed primarily through the use of one pair of codes, high impact and low impact (see Appendix F Section 6). In addition, narrative corresponding to all other codes in the codebook was utilized when relevant to capture experiences of actual control, perceived control, initial response, current response, compliance, alignment with standards, absoluteness, and legitimacy. Finally, an additional code, interest, was implemented in 
order to capture former providers' willingness or desire to start providing BIP services once more. The use of these codes provides insight into the extent to which standards impacted closed programs, as well as what social psychological processes may have played a role in subsequent decisions regarding the provision of BIP services.

Impact. The average impact ratio was $.37(S D=.38$; ranging from $0.00-.85)$, indicating that on average $37 \%$ of comments related to impact were indicative of high impact. While all five participants noted that the introduction of standards had a low impact on their program's decision to stop providing services, only three participants indicated that the standards had a high impact. These findings indicate that while three participants mentioned the standards did impact their program closure, all participants noted that the standards did not play a primary role in the decision to stop providing BIP services. Further, on average, nearly two-thirds of comments made by participants regarding impact indicated low impact. Examining the content of the high impact and low impact codes in combination (see Appendix F Section 6; see Table 9) reveals that former providers do not believe the standards were the primary or exclusive reason they stopped providing BIP services. Even those that reported that the standards had some impact qualified their statements to indicate that while standards did play a role, they were not the only reason. Thus, it appears that while the standards did impact the programs that these participants represented, the ending of BIP services was only partially the result of state standards and primarily due to outside factors (e.g., prioritization of other services, funding, etc.). Additionally, participant comments indicated that the majority of these former providers enjoyed the work and may be open to revisiting this work in the future. 
The remaining two participants did not make any comments, positive or negative, about the extent to which they liked the work or wanted to continue the work in the future. While elimination of batterer intervention services was primarily attributed to factors beyond state standards or to the standards in combination with other factors, the interviews were examined to determine whether former provider participants experienced social psychological processes that could help explain program closure.

Actual control. First, experiences of actual control were evaluated across the five former providers. The average actual control ratio was $.32(S D=.41$; ranging from 0.00 1.00 ), indicating that on average $32 \%$ of comments regarding actual control were indicative of high actual control. Two participants reported only experiences consistent with low actual control (actual control ratio $=0.00$ ) and one participant reported only experiences consistent with high actual control (actual control ratio $=1.00$ ). The remaining two programs reported experiences primarily aligned with low actual control, with actual control ratios of .25 and .33 . The diversity in actual control ratios is consistent with the variability observed for current providers. While this is the case, $50 \%$ of current providers reported primarily high actual control as compared to only $20 \%$ of former providers. This suggests that actual control may be a factor that contributes to program closure, as those who persisted in providing services reported more experiences of actual control. Though extremely underpowered, an independent samples t-test was conducted to determine if a statistically significant difference in actual control ratios for the current $(M=.46, S D=.52)$ and former $(M=.32, S D=.41)$ provider groups could be identified 
A significant difference was not detected, $t(16)=1.03, p=.32$. Thus, the difference in actual control among current and former providers is not statistically reliable.

Next, experiences of perceived control were not assessed because participants did not report experiences consistent with the codes associated with perceived control. Specifically, high and low perceived ability, and high and low procedural justice codes were not applied for former providers. The lack of codes relevant to perceived control is logical as these individuals likely do not think about whether or not they would be able to impact the standards and the fairness of the standards moving forward, as they are currently not involved in services affected by the standards. Thus, perceived control, as well as the extent to which actual control and perceived control are associated, could not be assessed for these five participants.

Negative attitude change and maintenance. While perceived control could not be examined, the change and maintenance of initial negative attitudes towards the standards were evaluated. After establishing initial and current response to the standards, the extent to which participants changed or maintained their initial negative attitudes was evaluated. Of the former providers, two who reported a positive initial response also reported a positive current response and one who reported a positive initial response did not provide comments related to current response. Only two participants reported initial responses that were primarily negative and thus only these two participants were evaluated for negative attitude change (i.e., a proxy for rationalization) and negative attitude maintenance (i.e., a proxy for reactance). Both of these participants reported primarily negative current responses, indicative of negative attitude maintenance. Each of these 
participants described that they initially had reservations or disagreement with the standards and these same reservations persisted. They did not indicate that their perceptions became more negative but rather they stayed consistently negative.

Absoluteness. Next, the extent to which former provider participants viewed the standards as absolute was examined. Absoluteness ratios were computed as was done for current providers. The average absoluteness ratio was $.53(S D=.51$; ranging from 0.00 1.00). This indicates that on average $53 \%$ of the comments are related to the presence of absoluteness as opposed to the absence of absoluteness (i.e., non-absoluteness). Two participants only reported perceptions aligned with absoluteness (absoluteness ratio = 1.00) and two participants only reported perceptions aligned with non-absoluteness (absoluteness ratio $=0$ ). The remaining participant reported views primarily consistent with absoluteness (absoluteness ratio $=.67$ ). The slightly higher proportion of participants reporting more perceptions and experiences consistent with absoluteness is consistent with what was observed in the sample of current providers. In both samples, most providers (60\% of former providers and $62 \%$ of current providers) viewed the standards as more consistent with absoluteness than non-absoluteness.

While absoluteness' role in changing negative attitudes towards the standards (i.e., a proxy for rationalization) could not be examined due to the lack of any former providers who changed their negative attitudes to be primarily positive, the impact of absoluteness was evaluated for the participants who maintained their negative attitudes towards the standards (i.e., a proxy for reactance). The two participants who were identified as having maintained their negative initial attitudes towards the standards had 
different views regarding absoluteness. One of these participants reported comments coded exclusively as non-absoluteness, while two-thirds of the second participant's comments were coded as indicative of absoluteness. This is similar to what was observed in the current provider interviews -- absoluteness did not reliably differentiate those who maintained their negative attitudes towards the standards (i.e., a proxy for reactance).

Legitimacy. Legitimacy in its complete form was not evaluated due to the lack of comments relating to both procedural justice and norms. Findings from the evaluation of policy logic indicate that former provider participants tend to view the standards as lower in policy logic than current provider participants. While this is the case, it is important to note that former providers did not discuss standards' alignment with best practices and instead focused their comments exclusively on whether or not standards are evidencebased. This may explain why more variability was seen in the current provider sample, where participants did have more discussion surrounding standards and their relationship to best practices. Additionally, former providers did not provide extensive information related to procedural justice and social norms and thus it appears they did not have as much input related to the legitimacy of the standards and standards creation process compared to the current providers.

\section{Research Question 3}

While gaining an understanding of the social psychological processes that contribute to compliance with state policy is important, obtaining a clear picture of implementation experiences is also vital. Specifically, obtaining knowledge regarding implementation experiences allows the opportunity to pinpoint areas that are problematic, 
areas of strength that can be capitalized upon, and explicit suggestions to improve the implementation process. In order to accomplish this, $R Q 3$ asks how have programs in Oregon have implemented the state standards. Five sub-questions were evaluated in order to gain a comprehensive appreciation for the implementation process and what could be done to aid providers in achieving compliance. These sub-questions assess implementation strategies, changes in policies and characteristics, enablers to compliance, barriers to compliance, and support needed to achieve compliance.

Implementation strategies. The first step in gaining a clear understanding of the implementation processes is to achieve a clear view of the actions that participants have taken towards compliance with standards. Research Question $3 a$ aims to accomplish this by asking which specific actions participants have taken to implement the standards. One code, implementation strategies (see Appendix F Section 7; see Table 9), was utilized to address $R Q 3 a$. Implementation strategies were named a total of 57 times $(M=4.38, S D=$ 3.04; ranging from $0-8)$ and nearly every participant $(n=12 ; 92 \%)$ described at least one strategy. These findings indicate that most participants were able to describe at least one action taken towards implementation. Further, of those that did describe at least one implementation strategy, several strategies were often explained.

The content discussed when participants were asked to describe the actions they have taken towards implementation (see Appendix F Section 7) provides insight to address $R Q 3 a$. Specifically, these experiences help determine what strategies programs have used to implement the standards. It appears that a variety of strategies have been utilized to implement the standards and increase compliance. These strategies include 
reading the standards, changing practices and policies, attending trainings, hosting trainings, building relationships, and hiring new staff (see Table 10). Each of these was described as a key step towards compliance that participants perceived as being important to position their program to better adhere to the standards. When these strategies are considered as a whole it appears that many of them revolve around collaboration with others, whether it is meeting collaboration requirements, or hosting or attending trainings. The ability to network with key stakeholders in the community collaborative response in order to build relationships and become aware of training opportunities has been a vital component of implementation for most providers. Beyond collaboration, some straightforward steps have also been taken in order to increase implementation. For instance, simply reading the standards has been beneficial for some participants. Additionally, participants discussed two strategies that may be time consuming or costly, but are somewhat straightforward. Specifically, changing policies and procedures, or hiring new staff were discussed as steps that have contributed to implementation. In sum, there are a variety of strategies towards implementation that participants reported using to comply with the standards. Most prevalent implementation strategies appear to center around collaboration, but other strategies, such as reading the standards, were also viewed as beneficial.

Difficulty and ease changing polices and characteristics. Participants described changes in practices and policies as one key strategy for implementation of the standards. In order to learn more about which specific components of the standards were easy to implement and which components were difficult to implement, $R Q 3 b$ was posed. 
Research question $3 b$ asks, what program policies and characteristics program representatives describe as being relatively more or less easy to change since they became aware of the standards. In order to address this research question, two codes were utilized; implementation ease and implementation difficulty (see Appendix F Section 8; see Table 9).

It appears that participants broadly believed their program had at least some qualities that did not require them to make substantial or challenging changes. When asked to name specific components of the standards that were easy to implement, participants indicated program length, aftercare, philosophy/curriculum, use of an accountability plan, community collaboration, training and co-facilitation (see Table 11), though the extent to which these components were discussed ranged from three to nine participants. Most commonly, participants indicted that program length and philosophical orientation were easy to implement. Conversely, less than one-quarter of participants indicated training, and mixed gender co-facilitation were non-problematic for implementation.

When asked to name specific components of the standards that were difficult to implement every participant named as least one component of the standards that their program has or continues to struggle to implement. These components included training, co-facilitation, philosophy/curriculum, community collaboration, program length, and the number of individuals per group (see Table 12). As was observed for components implemented with ease, the extent to which these components were discussed varied. 
Most commonly, difficulties were related to access to resources, such as training, staff, or community partners.

Together, this information addresses $R Q 3 b$ by highlighting the fact that components of the standards that are relatively simple for some programs to implement may be exceedingly difficult for other programs. Despite most participants' indication that the standards were easy to implement overall, every participant listed at least one component of the standards that was problematic for implementation or continues to be out of compliance. The component of standards listed most frequently as easy to implement was that of program length. While most participants reported that this component was relatively easy to implement, this was a problematic area for those who provide services in areas with conflicting local requirements. Fewer participants indicated that training and mixed gender co-facilitation were easy to implement and those who did endorse this sentiment reported already having program or staff features in place to facilitate adherence to this component of the standards. A minority of participants rather reported that training and mixed gender co-facilitation were areas they struggled with and these participants tended to be located in rural areas where obtaining training and finding qualified staff may be most difficult. Similarly, a subset of participants reported requirements surrounding group size were problematic. These participants were all representatives from relatively small programs that do not have a large number of groups to offer. Understanding which aspects of programs that were easiest and most difficult to change is important in order to identify where programs might need additional support. 
Next, participants were asked to provide their perspective on what factors have enabled compliance and what factors have served as barriers to successful compliance.

Enablers to compliance. Participants were asked to describe any enablers to implementation of and compliance with the state standards. This information was utilized to address $R Q 3 c$, which asks what have been the factors that aid implementation and compliance. In order to address this research question, two codes, enablers to compliance and social action research were utilized (see Appendix F Section 9; see Table 9). Factors that increased participants' ability to implement the standards were discussed a total of 53 times $(M=4.08, S D=3.52$; ranging from $0-11)$, with most participants $(n=11,85 \%)$ mentioning at least one enabler. Additionally, aspects of the research project that contributed to participants' ability to implement the standards were mentioned a total of 26 times $(M=2.00, S D=2.80$; ranging from $0-9)$. Participant responses included a variety of types of enablers and research project features (e.g., BIP surveys stimulate thought about practices and characteristics) that encouraged greater implementation (see Appendix F Section 9).

This collection of experiences provides great insight into $R Q 3 c$. Most participants were able to describe at least one enabler to compliance and among those who were able to identify at least one enabler, more than four enablers were named on average. This indicates that most participants perceive that multiple factors have contributed to their ability to implement and comply with the standards. Evaluation of interview responses indicated that 1. relationships with key partners, 2. activities such as attending trainings, completing local monitoring assessments, or contributing to the development of 
standards, 3. utilizing the content of the standards as a road map, and 4. program specific attributes such as within agency support, are all valuable for facilitating implementation and compliance with standards (see Table 13). Additionally, participation in the different phases of this research project, including the generation of the Oregon BIP Directory, completion of the survey, and participation in the interview were also viewed as valuable for implementation, as well as validation of the work being done by participants. While having an understanding of the variety of factors that have facilitated compliance is useful, in order to prevent difficulties or address current barriers to compliance, it is also necessary to determine what factors have made compliance difficult to achieve.

Barriers to compliance. In order to determine the barriers to compliance experienced by participants, analyses for $R Q 3 d$ aimed to identify barriers to compliance with the standards that have occurred. In order to address this research question, one code, barriers to compliance, was utilized (see Appendix F Section 10; see Table 9). A total of 105 barriers were described $(M=8.08, S D=5.12$; ranging from $2-21)$. The fact that every participant listed at least one barrier and on average listed eight barriers that have inhibited or made implementation and compliance more difficult indicates that participants are experiencing numerous barriers when attempting to implement standards. The content of these barriers (see Appendix F Section 10) was diverse and includes difficulties building relationships, completing required activities, understanding the content of the standards, and program features that make implementation problematic (see Table 14). It appears that many participants have experienced difficulties creating and maintaining collaborations. This stems from perceived lack of response from 
community partners, as well as negative experiences with attempted collaborations. Further, the BIP community itself is perceived to be problematic by some participants who believe it has become much more focused on business instead of ending violence against women. Participants also indicated that achieving trainings is difficult due to the lack of trainings in some areas, as well as the time and cost associated with traveling to trainings in other areas. In addition to barriers related to collaboration and required activities, the majority of participants reported that the content of the standards was problematic. Specifically, participants indicated that the lack of clarity regarding how much the LSA can change the requirements of the standards, as well as the formal language of the standards, caused difficulties with implementation. Finally, program factors including rural location, several participants discussed size, and lack of resources. It appears that the ability to meet different components of the standards may be more difficult when programs are far from other resources (e.g., collaborative partners or trainings), only see a small number of clients, or do not have sufficient funding to carry out all components of the standards (e.g., co-facilitation). The barriers highlight opportunities for intervention and support in order to build capabilities and increase implementation. In order to gain more concrete information about what participants' believe has been or would be helpful to facilitate compliance, the need for support was assessed.

Support Needed. Understanding the scope of enablers and barriers to compliance can aid in identifying what helps programs implement standards as well as what impedes implementation. In addition to cataloguing the various enablers and barriers to 
compliance, participants were asked to describe what support they viewed as crucial to comply with standards. This topic was assessed through $R Q 3 e$, which asks what needs do participants identify in order to successfully implement the standards? In order to address this research question, one code, needed support, was utilized (see Appendix F Section 11; see Table 9). Participants named a total of 68 suggestions related to the support they desired to better implement the standards $(M=5.23, S D=3.42$; ranging from $1-12)$. When examining participant suggestions (see Appendix F Section 11), several themes emerged (see Table 15). First, participants indicated that creating connections among BIP providers would be valuable. Suggestions to achieve this included developing a statewide association of providers, holding conferences for BIPs in Oregon, and creating a listserv to connect providers. Second, participants reported that creating a consistent monitoring system could be useful. Most participants who advocated this approach indicated that they believe the monitoring system should not be punitive but instead focus on program development and improvement. Third, participants suggested modifying the content of the standards to allow flexibility and increase comprehension. These ideas centered on developing additional resources to make the standards easier to understand and therefore follow. For example, one participant indicated a bulleted list of concrete requirements would be useful. Finally, participants indicated that increased financial resources would be beneficial, though they did not provide details on where this money would come from or how it would be allocated. Thus, creatively developing resources for programs may increase their ability to implement standards.

\section{Research Question Four}


While understanding the extent to which various social psychological processes and implementation strategies exist for the Phase Two sample as a whole, the current study also aims to understand how these experiences differ as a function of program compliance with state standards. Thus, $R Q 4$ asks, do the responses and reactions to standards differ for programs with different levels of compliance? In order to address this question, the subsample of high and low compliance program participants identified during Phase One of the current study was utilized. Experiences for the seven high compliance program participants and the six low compliance program participants were compared to address five hypotheses addressing the social psychology of compliance.

Actual control. First, the extent to which actual control differed in the high and low compliance groups was examined to speak to $H 4 a$. This hypothesis asserts that high compliance program participants will describe relatively more experiences of actual control as compared to low compliance program participants (see Table 16). In order to test this hypothesis, two codes, high actual control and low actual control, were utilized (see Appendix F Section 12). The average actual control ratio for the seven high compliance programs was $.65(S D=.41$; ranging from $0.00-1.00)$. This indicates that on average $65 \%$ of these participants' comments related to actual control were indicative of high actual control. Additionally, five of the seven high compliance participants reported more experiences of high actual control compared to low actual control evidenced by actual control ratios greater than .50 . The average actual control ratio for the six low compliance programs was $.36(S D=.22$; ranging from $0.00-.67)$. This indicates that on average $36 \%$ of these participants' comments related to actual control 
were indicative of high actual control. Further, only one of the six participants reported more experiences of high actual control compared to low actual control evidenced by an actual control ratio greater than .50 .

Thus, when experiences of actual control were examined descriptively, high compliance program participants on average have higher actual control ratios. Specifically, $65 \%$ of high compliance program participant comments regarding control were indicative of high actual control compared to $36 \%$ of low compliance program participant comments. Thus, high compliance program participants reported actual control ratios $57 \%$ higher than their low compliance program participant colleagues. Though extremely underpowered, an independent samples t-test was conducted to determine if a statistically significant difference in actual control ratios for the high $(M=$ $.65, S D=.41)$ and low $(M=.36, S D=.22)$ compliance groups could be identified. A significant difference was not detected, $t(11)=1.55, p=.15, d=1.21$. Thus, the difference in actual control among high and low compliance participants was not statistically reliable.

Assessment of the content of the interview data (see Appendix F Section 12) revealed that those who reported high compliance were much more deeply involved in their creation and refinement. Conversely, those who reported low compliance were not only uninvolved but also unaware of the standards creation and refinement process. Together, this information provides valuable insight necessary to address $H 4 a$. It appears that this hypothesis was partially supported, as there was both a descriptive difference in the quantity of high actual control and low actual control experiences, as well as 
differences in the quality of these experiences. However, this descriptive difference was not statistically reliable. High compliance program participants were more involved in the standards creation process, as well as more knowledgeable and descriptive regarding how the standards were developed compared to the low compliance program participants.

Perceived control. Next, the extent to which perceived control differed in the high and low compliance groups was examined to speak to $H 4 b$. This hypothesis asserts that high compliance program representatives will describe greater experiences of perceived control as compared to low compliance program representatives (see Table 16). Four codes (i.e., high perceived ability, low perceived ability, high procedural justice, and low procedural justice) were used to examine this construct (see Appendix F Section 12). Across the seven high compliance programs, the average perceived control ratio was $.47(S D=.30$; ranging from $0.00-1.00)$. This indicates that on average $47 \%$ of these participants' comments related to perceived control were indicative of high perceived control. Further, two of the seven participants reported more experiences of high perceived control compared to low perceived control evidenced by actual control ratios greater than .50. Additionally, two participants reported an equal number of high and low perceived control perceptions (perceived control ratio $=.50$ ). Across the six low compliance programs, the average perceived control ratio was.18 $(S D=.21$; ranging from $0.00-.43)$. This indicates that on average $18 \%$ of these participants' comments related to perceived control were indicative of high perceived control. All participants reported more experiences of low perceived control compared to high perceived control evidenced by actual control ratios lower than .50 . 
These findings indicate that descriptively high compliance program participants reported greater perceived control compared to low compliance program participants. Specifically, $47 \%$ of high compliance program participant comments regarding perceived control were indicative of high perceived control compared to $18 \%$ of low compliance program participant comments. Thus, high compliance program participants reported perceived control ratios $89 \%$ higher than their low compliance program participant colleagues. Next, though extremely underpowered, an independent samples t-test was conducted to determine if a statistically significant difference in perceived control ratios for the high $(M=.47, S D=.30)$ and low $(M=.18, S D=.21)$ compliance groups could be identified. A significant difference was not detected, $t(11)=2.01, p=.07, d=.93$. Thus, the difference in perceived control among high and low compliance participants was not statistically reliable.

This information coupled with the code content (see Appendix F Section 12) provides valuable insight to address $R Q 4 b$. It appears that there is partial support based on the large effect size and distinctions in the content of responses across high and low compliance participants, though this was not supported inferentially. Interview response content revealed a difference in familiarity with the standards process and key individuals for high and low compliance participants. Specifically, for high compliance program participants, the greater awareness of the standards process appears to have instilled a greater ability to plan how they would achieve desired changes, as well as greater confidence that their opinions will be taken seriously. Further, while the high compliance program participants described more instances of one component of perceived control 
(i.e., low procedural justice), this again appears to be the result of greater knowledge and awareness.

Negative attitude change and maintenance. Next, the hypotheses that high compliance program representatives will describe relatively more reactions consistent with change in negative attitudes towards the standards (i.e., a proxy for rationalization) and relatively fewer reactions consistent with maintenance of negative attitudes towards the standards (i.e., a proxy for reactance) as compared to low compliance program representatives were evaluated. To address each of these hypotheses, the frequency and content of four codes (i.e., positive initial response, negative initial response, positive current response, and negative current response) were utilized (see Appendix F Section 12). After establishing initial and current responses to the standards, the pattern of these responses was examined for evidence of attitude change (i.e., a proxy for rationalization) and attitude maintenance (i.e., a proxy for reactance). Negative attitude change is operationalized as experiencing a primarily negative initial reaction to the standards followed by a primarily positive current reaction to the standards. Negative attitude maintenance is operationalized as experiencing a primarily negative initial reaction to the standards followed by a primarily negative current reaction to the standards. As described in $R Q 2 c$ and $R Q 2 d$, four participants described shifting their negative attitudes towards the standards to be primarily positive and four participants described maintaining their negative attitudes towards the standards. The remaining five participants had a primarily positive initial response and thus were not examined further. In order to determine whether negative attitude change and maintenance is related to compliance, the four 
participants who shifted their negative attitudes to be primarily positive were examined to determine if they represented high or low compliance programs. Next, the four participants who maintained their negative attitudes were examined to determine if they represented high or low compliance programs.

Consistent with $H 4 c$, three of the four (75\%) participants who shifted their negative attitudes to be primarily positive (i.e., a proxy for rationalization) were representatives from high compliance programs. The remaining four high compliance programs reported an initial response to the standards that was primarily positive. This pattern supports the assertion that despite initially negative perceptions of the standards, those who have been able to achieve a high level of compliance were those who shifted their views towards the standards to be primarily positive. The one participant who did not fit the pattern of findings predicted by $\mathrm{H} 4 \mathrm{c}$ was an individual who, despite shifting their views to perceive the standards more positively, represented a low compliance program.

Also consistent with $H 4 d$, all four (100\%) of the participants who maintained negative attitudes toward the standards (i.e., a proxy for reactance) were representatives from low compliance programs. Again, this supports the notion that those who maintained their negative views of the standards may have a more difficult time making necessary changes or improvements in order to achieve compliance with the standards. These findings could not be tested statistically, due to the violation in the expected cell count associated with a chi-squared test. Specifically, greater than $20 \%$ of the cells had an 
expected cell count below 5, rendering the test inappropriate. Thus, descriptively $H 4 c$ and $H 4 d$ were supported.

Legitimacy. The final hypothesis assessed the extent to which high and low compliance program participants differ in their views regarding the legitimacy of the standards. Specifically, $H 4 e$ asserts that high compliance program participants will describe the standards and their creation as relatively more legitimate compared to low compliance program participants. In order to assess this hypothesis, six codes (i.e., high procedural justice, low procedural justice, positive norms, negative norms, high policy logic, and low policy logic) were examined.

After establishing the extent to which each component of legitimacy is present for high and low compliance program participants (see Appendix F Section 12), legitimacy overall was assessed by examining legitimacy ratios for these two groups (see Table 16). Across the seven high compliance program participants the average legitimacy ratio was $.37(S D=.23$; ranging from $0.00-.67)$, indicating $37 \%$ of the comments made regarding legitimacy supported perceptions of high legitimacy towards the standards. The majority of participants $(n=5 ; 71 \%)$ reported fewer perceptions of high legitimacy as compared to low legitimacy. Across the six low compliance program participants, legitimacy ratios ranged from $.07-.86$. The average legitimacy ratio was $.44(S D=.28)$, indicating $44 \%$ of the comments made regarding legitimacy supported perceptions of high legitimacy towards the standards. Fifty-percent of the low compliance participants reported fewer perceptions of high legitimacy as compared to low legitimacy, while one participant reported an equal number of high and low legitimacy perceptions, and the final two 
participants endorsed more perceptions in alignment with high legitimacy as compared to low legitimacy. Though extremely underpowered, an independent samples t-test supported the lack of difference in legitimacy in high $(M=.37, S D=.23)$ and low $(M=$ $.44, S D=.28)$ compliance program participants, $t(11)=-.48, p=.64, d=.27$. Thus, it appears that $H 4 e$, which proposed high compliance program participants would view the standards as more legitimate, was not supported. 


\section{Chapter 4: Discussion}

This dissertation addressed four primary research questions. First, the study aimed to determine the extent to which batterer intervention programs (BIPs) in Oregon have implemented state policy intended to determine program characteristics and practices. Second, to assess the frequency, valence, and extent of several social psychological constructs that may help explain the extent to which BIPs implement state policy. Third, to describe the range of implementation strategies and experiences in order to inform policymakers about BIP providers' experiences and offer recommendations to address difficulties experienced by providers. Finally, the study attempted to determine the extent to which the experience of actual control, perceived control, attitude change (including the potential explanations for change of rationalization and reactance), and legitimacy varied for high and low compliance program participants. Specifically, it was hypothesized that compared to low compliance program participants, high compliance program participants would report greater actual control, perceived control, and legitimacy over the standards. Further, it was hypothesized that participants from high compliance programs would be more likely to report changing initial negative attitudes towards standards (i.e., a proxy for rationalization), while low compliance programs would be more likely to report maintaining initial negative attitudes towards standards (i.e., a proxy for reactance).

\section{Summary of Findings}

Current program functioning. Overall, participants reported adhering to an average of $75 \%$ (ranging from 53\% to 97\%) of the assessed components of standards. 
Thus, every program reported compliance with at least half of the assessed components of the standards.

The extent to which implementation occurred for specific components of the standards varied. When program logistics were considered, most programs reported adherence to many of the logistical components of the standards. More than threequarters of programs reported having implemented portions of the standards related to group size, written victim safety policies, written criteria for program completion, written policies for ensuring victim/partner confidentiality, program length of at least 48 weeks, and program completion requirements. Between $25 \%$ and $75 \%$ of programs required written policies and procedures concerning program contact with victims, written policies for storing victim contact information, written policies and procedures regarding client transfers between programs, reported recording of the number of clients that complete the program after intake, and the utilization of mixed-gender co-facilitation for all groups. While most programs reported having the individual requirements for written policies and procedures met, when considered together, less than one-quarter of programs reported having all six written policies and procedures required by standards. Additionally, training requirements (i.e., victim advocacy and BIP training) for facilitators had been met for all facilitators in slightly more than half of the programs, while within programs about three-quarters of facilitators had completed the required trainings.

Examinations of adherence to the proscribed and prescribed intervention strategies also revealed that at least $80 \%$ of programs have implemented components of 
the standards related to intervention strategies. These included refraining from endorsing prohibited causes of battering (e.g., poor impulse control or anger) and refraining from endorsing prohibited intervention strategies (e.g., encouraging ventilation techniques or couples counseling). Additionally, $80 \%$ of programs reported utilizing all approved intervention strategies outlined in the standards (e.g., use of respectful confrontation and address tactics used to justify battering). While most programs reported implementation of the components of standards related to intervention strategies, it is important to recognize that a significant minority of programs reported prohibited views regarding the cause of battering, the use of prohibited intervention strategies, and the lack of required intervention techniques.

Next, policies related to victim and partner contact were considered. More than three-quarters of programs reported that only individuals deemed appropriate by the standards have access to participant contact information. While this is the case, more than one-quarter of the programs reported contacting victims or partners in prohibited circumstances (e.g., to solicit information about how a client is doing in the home). Additionally, less than $75 \%$ of programs reported distributing information to victims and partners and only $40 \%$ of the programs that distribute information include all types of information recommended by the standards (e.g., information about safety planning and limitations of BIP outcomes). Further, of these programs, only slightly more than onethird offered this material in languages other than English.

Finally, the extent to which programs report implementation of the community collaboration components of the standards was investigated. More than $90 \%$ of programs 
reported having contact with a victim advocacy program and nearly all of these programs reported having a staff member who serves as a liaison to the advocacy agency. Fewer programs, though still close to two-thirds, indicated that a victim agency has reviewed their policies and procedures. Additionally, more than $80 \%$ of the programs that reported being in an area with a DV council indicated having a staff member who regularly attends DV council meetings. When the criminal justice system was considered collaboration remained common. The high rate of collaboration with the criminal justice system is not surprising as the criminal justice system serves as the primary referral source for most, if not all, programs. Nearly every program reported having a liaison to the criminal justice system and nearly three-quarters of programs reported contact with the local supervisory authority (LSA). Most programs reported program outcome and attendance information to these bodies, though nearly one-third reported only program outcome or attendance, or did not report either type of information. Additionally, most programs reported collaborating with other BIPs but fewer than half indicated they were part of a larger BIP organization or participated in the community to raise awareness about IPV.

Response to state standards. The second research question asked how participants responded to state standards by identifying the processes underlying their responses. Participant interviews were evaluated to identify reactions and responses to the standards indicative of actual control, perceived control, negative attitude change and maintenance, and legitimacy. The extent to which responses to standards were consistent with the operationalization of these constructs was examined, as well as the depth and 
content of these experiences. This was accomplished through the utilization of quantitative and qualitative analytic techniques to make sense of interview data. Additionally, $R Q 4$ assessed the extent to which responses consistent with these constructs differentiated high compliance and low compliance programs. Again, this was examined using quantitative and qualitative approaches for interpreting the interview data.

Actual control. Most participants described at least one experience of having actual control over the standards and one experience of not having control over the standards, indicating that participants may believe they had actual control over some portions of their experience and lacked actual control in other portions of their experiences. Thus, actual control was not an all or nothing phenomenon. When considered together, an average of $46 \%$ of comments related to actual control represented participants having been actively involved with or knowledgeable about the development and refinement of the standards. Conversely, 54\% of comments represented participants being uninvolved with or unknowledgeable about the development and refinement of the standards. Thus, experiences of not having actual control were more common than experiences of actual control. When the content of these experiences was examined, interesting patterns emerged. It appears that having actual control over the standards is closely tied to direct participation on the Standards Advisory Committee. The participants who were members of this committee reported higher frequencies and more varied examples of actual control. Those who were not on the Standards Advisory Committee but described experiences of having actual control tended to be individuals who were in contact with someone from the Standards Advisory Committee. Thus, participation on or 
close relationships with members of the Standards Advisory Committee appears to be an important aspect of the descriptions of actual control. Conversely, those who more commonly reported not having actual control over the standards were individuals that lacked connections to and knowledge about the Standards Advisory Committee.

It was hypothesized that experiences of actual control would be greater among compliance program participants compared to low compliance program participants. Despite the large effect size $(d=1.21)$, a statistically reliable difference was not detected. While this is the case, gaining an understanding of the more specific content of the interview data helps describe the nuances in actual control experiences across high and low compliance program participants. High compliance program participants primarily voiced comments indicative of having actual control over the standards (actual control ratio $=.65)$, while low compliance program participants primarily voiced comments indicative of not having actual control over the standards (actual control ratio $=.36$ ). Review of interview material revealed that all three participants who serve or have served on the Standards Advisory Committee are members of the high compliance group. Further, the other members of the high compliance group were aware of the standards creation process and Standards Advisory Committee. In contrast, participants from the low compliance group were not members of the Standards Advisory Committee and did not have a clear sense of how or who developed the standards.

Perceived control. Across participants, descriptions consistent with both the presence and absence of perceived control were discussed. On average, $66 \%$ of the 
comments made related to perceived control were indicative of participants perceiving a lack of control over the standards.

As was seen with actual control, all of the individuals who are or have been members of the Standards Advisory Committee reported perceiving control over the standards moving forward. Beyond these participants, others who made statements indicating they perceived having control tended to report knowing who they would turn to if a concern arose or indicating they are confident in themselves that they could induce change if desired. Further, after accounting for those who serve or served on the Standards Advisory Committee, only a small minority of participants reported they perceive the standards process as fair and/or the Standards Advisory Committee as representative of most providers.

Participants' comments about perceived control most often indicated a lack of influence on the standards. The content of these comments varied but themes that were discussed by multiple participants included lack of confidence regarding whom to raise concerns with, questions as to whether their concerns would be taken seriously, and lack of adequate representation on the Standards Advisory Committee. Participants discussed a lack of knowledge about the identity of key individuals in the standards community. Additionally, participants discussed the political climate of the IPV community and how tensions and competition in the community have impacted their perceptions related to being taken seriously when issues arise. Finally, numerous participants questioned the representativeness of the Standards Advisory Committee in terms of race/ethnicity, sexual orientation, class, role in the community collaborative response, and geographic 
program location. Overall, perceived control over the standards appeared to be limited in the sample.

It was hypothesized that high compliance program participants would describe greater experiences of perceived control compared to low compliance program participants. Despite the strong effect size $(d=.93)$, this hypothesis was not supported inferentially. This being the case, the content of the interview data is still informative to better understand the experiences of actual control in these two groups. While both groups primarily reported experiences consistent with perceived lack of control, this deficit was most prominent for low compliance program participants. Specifically, high compliance program participants reported perceived control in $47 \%$ of their comments related to this construct, while only $18 \%$ of the low compliance program participant comments related to perceived control were indicative of having perceived control. Review of the interview data revealed that high compliance program participants discussed being familiar with the content of the standards and key individuals related to the standards. Conversely, low compliance program participants discussed being unfamiliar with the standards and those that they could contact in order to impact the standards. This familiarity reported by high compliance program participants contributed to them feeling more confident that they would be able to impact the standards if desired.

Actual and perceived control. Finally, it was hypothesized that high actual control participants would report greater perceived control compared to low actual control participants. Those who reported higher actual control had a perceived control ratio $23 \%$ higher than those who reported lower actual control but this difference was not 
statistically reliable. Specifically, there was not a significant difference in perceived control ratios among those who primarily reported experiences of actual control versus those who primarily reported experiences lacking actual control. Further, in both the high and low actual control groups, perceived control ratios were below .40. This indicates that across both groups, less than $40 \%$ of the comments related to perceived control were indicative endorsements of perceived control.

Negative attitude change and maintenance and absoluteness. Of the eight participants who had an initial response ratio below .50 , indicative of a primarily negative initial reaction to the standards, four had a primarily positive current response to the standards, indicating rationalization may have occurred, and four had a primarily negative current response to the standards, indicating reactance may have occurred. Those whose reported changing their initial negative attitudes towards the standards tended to voice apprehension when the standards were introduced followed by greater acceptance of the standards after becoming more familiar with the content. These participants initially questioned the content of the standards and were fearful about the ways in which the standards would be enforced. As they learned more, their anxiety about the standards decreased and ultimately, they described their current views towards the standards as primarily positive. Those whose reported maintaining their initial negative attitudes towards the standards tended to have significant concerns about the content of the standards initially, which persisted over time. These participants initially had specific concerns about the content of the standards, such as the lack of flexibility, as well as the feasibility of complying with the standards. The negative perceptions 
persisted and when participants were asked to describe their current view of the standards, most comments focused on aspects of the standards with which they disagree (e.g., exclusive focus on male batterers, lack of flexibility, etc.).

Next, the construct of absoluteness was introduced in order to differentiate those who changed their negative attitudes (i.e., a proxy for rationalization) and those who maintained their negative attitudes (i.e., a proxy for reactance) among participants. It was hypothesized that those who changed their initial negative attitudes would view the standards as primarily absolute, while those who maintained their initial negative attitudes would view the standards as primarily non-absolute. In order to evaluate this hypothesis, the frequency and content of experiences of absoluteness and nonabsoluteness were examined within the negative attitude change and negative attitude maintenance groups. Within the negative attitude change group 58\% of comments discussing the extent to which the standards are absolute were indicative of absoluteness versus non-absoluteness. Within the negative attitude maintenance group $71 \%$ of the comments discussing the extent to which the standards are absolute were indicative of absoluteness versus non-absoluteness. When the content of comments were examined, it was determined that the negative attitude change and negative attitude maintenance groups did not meaningfully differ with regards to the types of comments made relating to absoluteness, though the negative attitude maintenance group made a greater number of comments related to absoluteness. Thus, this hypothesis was not supported. Regardless of group, most participants viewed the standards as primarily absolute and the negative attitude maintenance group voiced relatively more comments indicative of absoluteness. 
Next, it was hypothesized that those who changed their initial negative attitudes towards the standards (i.e., a proxy for rationalization) would be more likely to represent high compliance programs while those who maintained their initial negative attitudes towards the standards (i.e., a proxy for reactance) would be more likely to represent low compliance programs. Both of these hypotheses were supported in the current study. Specifically, $75 \%$ of the participants who changed their initial negative attitudes were representatives from high compliance programs and $100 \%$ of the participants who maintained their initial negative attitudes were representatives from low compliance programs.

Legitimacy. Across all participants, $40 \%$ of comments related to legitimacy indicated the participants believed the standards creation process and authority body was legitimate, while $60 \%$ of the comments indicated that participants did not believe the process and body were legitimate. In this study legitimacy was comprised of three components, procedural justice, norms, and policy logic. Participants tended to view the policy standards as somewhat low in procedural justice; only $30 \%$ of comments related to procedural justice reflected perceptions that endorsed the standards creation as being fair. When norms were considered it was evident that participants tended to make a limited number of comments related to norms and only seven participants (54\%) discussed either positive or negative norms surrounding the standards. Of those that did make a comment related to norms, these comments were nearly half positive and half negative. More commonly, participants reported discussion about standards in the field but did not feel that the discussion was particularly positive or negative. Finally, examination of policy 
logic revealed that comments were nearly split with $48 \%$ of comments related to policy logic being indicative of having policy logic and $52 \%$ being indicative of lacking policy logic.

Comments reflecting legitimacy included statements that the Standard Advisory Committee was inclusive, that the standards were viewed positively among providers, and that the standards were based in best practice. Comments reflecting lack of legitimacy included statements that the Standards Advisory Committee lacked diversity, that the field of providers views the standards negatively, and that the standards were not created from evidence-based practice due in large part to the lack of research in the field. Thus, across all participants, a lack of perceived legitimacy was observed. Given the low degree of procedural justice and relatively equal experiences of social norms and policy logic, it is not surprising that overall participants viewed the standards as primarily nonlegitimate as opposed to legitimate.

It was hypothesized that high compliance program representatives would describe the standards as more legitimate compared to low compliance program representative's. The current study did not support this hypothesis. While the difference in legitimacy ratios was not significantly different across high and low compliance participants $(d=$ .27), contrary to expectations, high compliance program representatives made relatively fewer comments endorsing legitimacy (legitimacy ratio $=.37$ ) compared to low compliance program representatives (legitimacy ratio $=.44$ ).

Former providers. In addition to exploring the social psychological phenomenon among current providers, these experiences were also examined among former providers. 
The final sub-research question for $R Q 2$ inquired about the presence of these social psychological constructs, as well as impact of the standards overall, for providers who are no longer offering services. Interviews with former providers revealed that by and large participants did not view the standards as the primary or exclusive reason for terminating BIP services. On average, only $37 \%$ of comments related to the impact of standards were indicative of a high impact. Further, only three participants made at least one comment indicating high impact of the standards while all five participants made at least one comment indicating low impact of the standards. All participants who reported at least one comment reflecting the high impact of the standards did so while qualifying that the standards were one of several reasons for no longer offering BIP services. Most commonly, the reason for ceasing BIP services was logistical (e.g., finances, prioritization of other services). Further, most former participants (60\%) indicated they would be interested in re-introducing BIP services into their program once again if it were possible.

After establishing that the standards were not the primary rationale for program closures, the social psychological phenomenon assessed among current provider participants were evaluated. First, actual control was examined. Similar to what was observed among current providers, participants tended to report fewer instances of having actual control as compared to not having actual control, as evidenced by an actual control ratio of .32. Comments related to actual control indicated that these providers often did not participate in the standards process, though some did have an awareness of the standards creation process. 
Next, the extent to which negative attitudes towards the standards changed (i.e., a proxy for rationalization) or were maintained (i.e., a proxy for reactance) were assessed. The initial response to the standards was primarily positive for these providers, with $67 \%$ of comments related to initial response indicating a positive response. This was higher than what was observed in the sample of current providers (52\%). Comments related to initial response indicated that the former provider participants understood the reasoning for the standards and were generally supportive, though two participants noted disagreement. Current perception towards the standards was slightly less positive than the initial response. Specifically, $57 \%$ of comments related to current response were reflective of a positive current response. This was slightly higher than what was observed among current providers (44\%). While this is the case, given the difference in circumstance for current and former providers, current responses may be qualitatively distinct. For instance, some former providers responses suggested they might not have thought about the standards in several years. Additionally, their responses related to current perceptions of the standards were much more succinct and less detailed than what was observed for current providers. While, former provider participants may represent a different type of current perception, it is still interesting to note that participants tended to indicate that their responses had not shifted and what they viewed as positive and negative initially had stayed constant.

This information was used to examine negative attitude change and maintenance. Only two participants had initial responses to the standards that were primarily negative and thus only these participants were examined. Of these participants, both reported 
primarily negative current responses. This indicates their negative attitudes persisted, which may possibly be due to the experience of reactance or other factors (i.e., lack of exposure to the standards). Perhaps due to the desire to maintain freedoms indicative of reactance, $40 \%$ of those who ceased providing services have maintained their negative attitudes towards the standards (i.e., a proxy for reactance) while no former provider participants reported changing their negative attitudes to become primarily positive (i.e., a proxy for rationalization). It is possible that while these providers do not view the standards as having a large impact on their program closure, the negative perceptions towards the standards contributed to their decision to stop services.

Next, perceptions of absoluteness among former providers were identified. Approximately half of the comments related to absoluteness made were indicative of viewing the standards as absolute. This was similar to what was observed among current providers. The content of these comments points to the perception that compliance with the standards was valued and expected, though questions were raised as to the breadth and depth of knowledge about the standards held by community partners. Absoluteness was examined among the two providers who maintained negative attitudes towards the standards. As was observed in the current provider participants, a consistent pattern that aligns with expectations was not observed. Specifically, one former provider who maintained negative attitudes towards the standards viewed the standards as non-absolute while the other viewed the standards as absolute.

Finally, legitimacy was evaluated within the sample of former providers. The three components of legitimacy were not evenly represented in former provider 
interviews. Specifically, there were no comments related to procedural justice and only one comment related to norms. Thus, legitimacy was almost exclusively evaluated through the discussion of policy logic. This provided a proxy for legitimacy but it is important to recognize that this limited operationalization is flawed, as it does not include all aspects of legitimacy. Across all participants, $80 \%$ reported believing the standards did not have policy logic exclusively, and $20 \%$ reported believing the standards do have policy logic exclusively. This was different than what was observed among current providers. Current providers tended to discuss the standards as based in best practice but not in evidence, while former providers discussed just one aspect or viewed them as synonymous. As was observed in the sample of current providers, the content of comments related to policy logic indicated that standards are not based on evidence because evidence does not yet exist.

Implementation. The third research question identified the process by which BIPs in the state of Oregon have implemented state standards. This question assessed the strategies utilized to achieve implementation, changes made in the interest of implementation, enablers and barriers to achieving compliance, and support desired to allow a more thorough implementation. The breadth and depth of discussion related to implementation was accomplished through the utilization of quantitative and qualitative assessment techniques.

Implementation strategies. In order to successfully implement the various components of the standards, participants described many strategies that were utilized. Nearly every participant (92\%) provided at least one strategy they have utilized to 
encourage implementation of standards in their program. These strategies included making changes to program practices or policies (54\%), attending trainings (45\%), attempts to create or improve collaboration (38\%), thoroughly reading the standards (38\%), hosting trainings (23\%), and hiring of additional staff (15\%).

Difficulty and ease changing policies and characteristics. During the course of the interview, participants described components of their programs that were easily modified to achieve greater implementation, as well as components that were difficult to implement or remain out of compliance. Changes that were achieved easily and changes that were more difficult were identified at a similar frequency with most participants (92\%) describing components that were easy to change as well as components that were or remain difficult to change.

When participants described changes in program policies and characteristics that were relatively easy to enact it was clear that numerous components of the standards had already been integrated or were relatively simple to integrate into current program functioning. Specific components that were described as easy to implement included program length (69\%), program philosophy or curriculum (54\%), collaboration (38\%), mandating an accountability plan (23\%), achieving training requirements (23\%), mixed gender co-facilitation (23\%), and mandated aftercare (17\%). Further, the majority of participants (69\%) made an overarching statement indicating that overall standards have not been difficult to implement. While there were numerous components that participants believed were easy to implement, participants also reported that many components were difficult to implement or remain out of compliance. Relatively difficult components to 
implement included achieving training requirements (54\%), mixed gender co-facilitation (54\%), program philosophy or curriculum $(31 \%)$, program length $(23 \%)$, collaboration (23\%), and maintaining the standards mandated maximum number of participants per group (23\%). Additionally, two participants (15\%) indicated that the standards overall were difficult to implement.

Implementation enablers and barriers. After establishing which program characteristics have been relatively easy and difficult to change, participants were asked to describe factors that enabled implementation. Most participants $(85 \%)$ voiced at least one implementation enabler and many participants described multiple implementation enablers that they had experienced. Participants reported a variety of enablers including relationships with agencies, participation in activities, and program characteristics. Agency facilitators primarily included references to relationships with collaborative partners, such as corrections, DV councils, and BIP provider organizations, that have been vital in ensuring implementation occurs. Enabling activities included participation in relevant organizations, such as the Standards Advisory Committee, participation in trainings, and local monitoring of program characteristics. Participants also described qualities of their program or staff, such as personal knowledge of the standards as they were developed, within agency support for implementation, and personal qualities such as expertise or confidence in abilities, which enabled implementation. A minority of programs also indicated that the way in which the standards were written provided guidance and enabled implementation. Additionally, an unexpected enabler arose over the course of the interview process. Nearly half of the participants (46\%) made at least 
one comment indicating that the research process was an enabler to implementation. These comments included references to the researcher's outreach necessary to create the BIP Directory, the BIP survey, and the interview process.

As was observed when enablers to implementation were examined, barriers to implementation were numerous and varied. Every participant described at least one barrier to implementation and on average participants reported more than five barriers. The barriers to implementation described by participants included relationships with relevant agencies, difficulties achieving required activities, the content of the standards, and features of the program. Agency barriers included problems developing or maintaining required collaboration with collaborative partners. Participants described difficulties working with diverse partners including other BIPs, victim advocacy agencies, and the local LSA. Activity barriers were related almost exclusively to meeting the components of the standards related to mixed gender co-facilitation and training. Content barriers were described by over half of the participants and included comments indicating that the way in which the standards are written make them difficult to implement. For instance, the formal language and lack of clarity regarding the role of the LSA posed problems in interpretation for some participants. Finally, program barriers were voiced indicating that participants felt factors such as being from a rural location, small program or not having sufficient funding were problematic for implementation.

Support needed. After discussing the enablers and barriers to implementation, participants were provided the opportunity to brainstorm suggestions as to what could be done to encourage implementation for their and other programs. Participants were 
innovative and synergistic in their suggestions, providing a thorough list of possible options that could be undertaken to improve implementation statewide. When suggestions from all 13 participants were considered together, several categories of recommendations emerged. Categories of suggested changes include changes to the standards, collaborative relationships, monitoring and enforcement, and funding.

The most prominent category of suggestions centered on changes to the standards. Many of these comments stemmed from a lack of clarity as to what the standards required because they are difficult to understand for some participants. These participants indicated that a version of the standards written in succinct and clear language would be helpful. The next most common category of suggestions focused on collaborative relationships and was primarily voiced by participants in rural locations. This is perhaps not surprising given that participants identified positive collaborative relationships as an enabler to implementation and noted that problematic or lack of collaborative relationships inhibits implementation. Most suggestions regarding collaborative relationships were rooted in the desire to increase collaboration among providers. Participants went beyond simply noting that more collaboration would be helpful and instead provide concrete suggestions about how to increase collaboration among various agencies statewide. These suggestions included the creation of a statewide BIP network or professional organization, a conference for Oregon BIP providers, and resources to enable better communication among providers (e.g., e-mail listserv).

In addition to suggestions related to the content of the standards and relationships, about one-third of participants recommended introducing some type of formal monitoring 
or enforcement of standards. Across these participants the extent to which they believed monitoring should occur and recommendations related to the severity of outcomes associated with non-compliance differed. Most participants requested supportive monitoring to help guide programs in their process of implementation and one participant requested a formal certification process that eliminates providers who are not meeting the standards. The final support for implementation suggested by participants centered on the lack of funding for BIP services. While increased availability of funding would be valuable for programs, participants did not discuss where this funding might come from or how programs would be eligible for funding.

\section{Limitations}

As with any study there are several limitations inherent to the current study that must be taken into consideration when interpreting the implications of the findings. First, this study is contextually specific to the state of Oregon and findings may not be transferable to other states. Limited transferability (Lincoln \& Guba, 1985) may be the result of several factors. One factor is the variation in the content and enforcement of standards between states. As was demonstrated by Maiuro and Eberle (2008), while there are some commonalities among states there are also numerous differences that make the content of standards in each state unique. Oregon does not utilize a statewide enforcement or monitoring systems. In regards to compliance and implementation, this creates a different atmosphere than would be expected in a state that formally requires compliance. For example, states such as Washington, Kentucky, Colorado, and Virginia carry out some type of certification or accreditation process to ensure programs meet the 
components of the particular state's standards. States that utilize certification or accreditation procedures may require programs to complete applications documenting program practices and staff qualifications. These applications are then reviewed by as elected agency such as a government agency or community coalition. For providers, the benefit of this review process is that programs that achieve certification may be labeled as such or placed on a preferred providers list. For the community, this process ensures that all certified programs are adhering to state regulations.

Providers in states that utilize a certification process may have different experiences and reactions than those in Oregon. Specifically, if programs are unable to function successfully and receive referrals without certification, the role of the various psychological constructs examined in this study may not be related to compliance. Instead, these programs may comply with standards to maintain referrals, despite lacking actual or perceived control or maintaining negative views towards the standards. While this may be the case, little is known about the extent to which other states actually implement the certification processes they have in place. Thus, in states where certification does exist but little attention is paid to ensuring the accuracy of reported practices, reactions towards standards and experiences in implementation may be similar to what was observed in Oregon.

Future research should attempt to better understand the relationship between reactions to standards, the content of standards, and formal enforcement. To achieve this, a useful example is the state of Washington. Due to the proximity of Washington to the state of Oregon, several participants discussed the Oregon state standards and compared 
them to the Washington state standards. It became evident that despite physical proximity, the standards in Washington are quite different, with distinct training, length, and certification requirements. These differences may make it difficult to compare experiences relating to implementation across the two states, as there are different expectations and pressures to comply. While this is the case, it would be interesting to identify the various social psychological processes at play in a state like Washington with formal enforcement criteria and compare those reactions and responses to a location without statewide monitoring, such as Oregon. This would provide interesting insight into how rigidity of monitoring and enforcement impact perceptions and reactions including actual and perceived control, attitude change and maintenance, absoluteness, and legitimacy. For example, while the quality of these reactions may be similar across the two locations, it is possible that they are not helpful in differentiating compliance, as all programs would be expected to achieve a certain degree of adherence to standards.

A second factor that may impact transferability is the fact that each state has a unique history that led to the development and implementation of its standards. These unique histories may make it difficult to determine the extent to which the findings of the current study will transfer across the United States. In Oregon, the standards were adopted in 2006 and have been part of the BIP environment for seven years. The length of time a state has utilized standards may impact how program representatives perceive and implement standards in different states. In areas where standards are relatively new, program representatives may be just beginning the process of learning about the components of standards and determining what the regulations mean for their program. 
Conversely, in areas where standards have been in place for many years, providers may feel more comfortable with the various components and knowledgeable about how the standards impact their program. These differences may influence perceptions and comfort towards the standards and their various components.

An additional limitation of the study is the reliance on self-report assessments. All survey and interview data were generated through self-reports and may not be entirely accurate. Additionally, because the survey was designed and administered in collaboration with the Standards Advisory Committee, programs may have felt inclined to respond to the survey in a socially desirable way that aligns with the state standards. Though enforcement and monitoring does not occur on a statewide level in Oregon, participants may have thought that their ability to obtain referrals could be jeopardized if their program is perceived as one that does not adhere to the standards. If participants felt this way, they may have altered their reports to align more closely with the standards and the goals of the Standards Advisory Committee. In addition to the logical reasons participants may be motivated to report greater adherence to the standards, a metaanalysis examining reported compliance with policy found that self-reports tend to be biased towards greater adherence to policy when compared to objective measures (Adams, Soumerai, Lomas \& Ross-Degnan, 1999). Interestingly, this study found that the areas that were not overestimated included areas in which the topic was sensitive or when individuals were unaware of the current guideline (Adams et al., 1999). This finding suggests the plausibility of more accurate reporting of program practices if the 
participants view the standards as a sensitive topic or are not knowledgeable about the content of the standards.

Support for the validity of self-reports of BIP practices was demonstrated in the findings from a previous study, which found varying levels of reported compliance despite survey development and administration involvement by the Standards Advisory Committee (Boal, 2010). Variability demonstrates that programs are reporting compliance with some components of the standards and non-compliance with other components, suggesting that self-reports reflect actual practices rather than simply an endorsement of having achieved all components of standards. Specifically, in 2008 average compliance ranged from .25 to $1.00(M=.72$ and $S D=.22)$ when only four components were examined. Additionally, the current study, which included many more aspects of compliance than were included by Boal (2010), found average compliance ranged from .53 to .97 .This variation suggests that even if participants bolster their selfreports, they continue to report practices and characteristics that do not align with the standards.

In the context of the interviews, every attempt was made to ensure confidentiality and build rapport. The researcher initiated contact with all participants in order to foster trust and continuity of the participant-researcher relationship. The researcher met the participants in person at a location of their preference, which was intended to make the interview as comfortable and convenient as possible. Participants were ensured that only personnel on the research team would see their interviews in their entirety and no identifying information would be published. Participants were also given the opportunity 
to review their interview transcripts and review or modify any passages with which they did not feel comfortable. During the course of the interviews, all but one participant was extremely open and verbose, which appeared to indicate comfort and openness. The one participant who did not seem as engaged was not feeling well but insisted on completing the interview. Thus, it is difficult to determine whether their relatively brief responses were due to disinterest or mistrust versus simply not feeling well. Additionally, it is worth noting that participants shared many sensitive details about their views of the standards and the workings of their program, some of which they asked to be omitted from the interview transcripts. While these direct quotes are not available for analysis, their existence highlights the fact that during the interviews most participants were open and forthcoming.

Reliance on a single individual as the representative for each program could limit the validity of reports because different staff who are responsible for implementing policies and practices in BIP groups may have differing experiences implementing standards, perceptions of the content of standards, needs to encourage compliance, barriers to compliance, and enablers of compliance. While this may be the case, BIP directors were permitted to select representatives who would serve as the best source of knowledge regarding the implementation of standards. Four individuals who did not serve as program director were selected to complete the interview. Three of these participants represented programs that offer diverse services in addition to their BIP component (e.g., drug and alcohol programming), and the representative who participated was integral to the BIP component of the program. The final non-director participant was 
nominated because the program director could not attend the interview as planned. In this case, the program manager completed the interview and the program director reviewed the interview transcript and provided additional information when necessary. This ensured that participants in Phase Two of the study were likely to be most familiar with the programs' policies related to standards and how they evolved over time. This allowance produced a sample of participants who served in different roles in their program including program directors, managers, and lead facilitators. Because directors were permitted to nominate a program representative who is most knowledgeable about standards within the program, the current sample includes individuals responsible for diverse components of the standards including the need to hire new staff to allow cofacilitation, the creation of written program policies and procedures, and collaboration with community partners, such as probation and victim advocacy agencies. Additionally, it was common for participants to have dual roles (e.g., program manager and facilitator), making it likely that some participants may experience the impact of standards from a variety of perspectives.

An additional limitation relates to the extent to which the findings from Phase Two of the current study can be generalized to all BIPs in Oregon. This limitation stems from the use of purposive sampling in Phase Two. Specifically, it is possible that because individuals who were most extreme in terms of compliance were selected, their experiences may not align with the average program. While the use of purposive sampling for extreme cases may make generalization more difficult, this sampling choice was made based on the strengths that purposive sampling brings to the study. 
Specifically, purposive sampling for extreme cases ensured that participants with variation in their success implementing standards were included in the sample. This sampling technique was valuable because it ensured variability in program compliance such that a description of perceptions, reactions, and experiences were gathered from individuals that have had more and less success in implementation. Further, the segmented sample provided the additional feature of creating high and low compliance groups for comparison. Finally, while there may be limitations to generalizability related to Phase Two of the study, Phase One did attempt to sample all BIPs in the state of Oregon, thus increasing the ability to generalize the Phase One findings across the state.

The study design was useful in describing the extent to which compliance has been achieved and explaining the reactions that occurred during the process of implementation. While the selected design was immensely valuable, there are limitations that were introduced. These limitations are not uncommon in the study of policy implementation as complexities and nuance inherent in studying a dynamic and evolving implementation process tend to make experimental designs that account for all confounding variables extremely difficult, if not impossible (Meyers, Durlak \& Wandersman, 2012). Because this study investigated the real-world phenomenon of policy implementation in real time, only retrospective accounts of past attitudes and beliefs could be attained. This is especially relevant when considering the constructs of rationalization and reactance as these constructs require knowledge of attitudes preceding and following the introduction of a policy. The current study could not obtain attitudes and perceptions prior to the introduction of standards because they were introduced in 
2006. Thus, this study had to rely on retrospective accounts of attitudes and perceptions. This may have introduced an important limitation as retrospective accounts may differ from those provided in real time. While this is the case, established assessment tools, which allow for retrospective recollections (e.g., Dillard \& Shen, 2005), were used to develop interview questions. Additionally, it is valuable to note that initial responses to the standards were described frequently and were varied, suggesting that these retrospective accounts may still shed light on the general perceptions individuals had towards standards when they were introduced.

Given that there is a significant limitation inherent to the current study due to the use of attitude change or lack of change as proxies for the processes of rationalization and reactance, it is important to explore the diversity of explanations for the observed findings. The current study focused on the change and maintenance of initial negative attitudes toward the standards and used this information to provide a proxy for rationalization and reactance. Thus, while speculation as to the role of rationalization and reactance is introduced and discussed based on the literature available related to these constructs, it is important to note that these constructs only provide two potential explanations for the patterns in attitudes towards the standards observed in the current study. It is possible that additional factors may have influenced initial and current attitudes towards the standards. First, participants who reported changes in their attitudes towards the standards sometimes attributed this shift to increases in familiarity with or education about the standards. It is plausible that shifts in attitudes to view the standards more favorably were achieved through these processes rather than through the process of 
rationalization. If this is the case, the findings suggest that if increased compliance is important, developing opportunities to become more acquainted with the standards and learn more about their specific components may be immensely important. Second, participants who reported that their attitudes were consistently negative tended to discuss specific components with which they disagree. It is possible that these participants did not undergo reactance but instead have maintained their negative attitudes due to the specificity of their attitudes. Some of these participants also mentioned the lack of evidence-based practice in the BIP field and speculated that given the lack of evidence there may be a need for greater latitude to make professional judgments. This points to a different rationale for maintenance of negative views. Specifically, since the standards were enacted there has been little growth in the evidence-base for BIP practices. Greater familiarity or education about the standards would not change the fact that this evidencebase is lacking and it is possible that this lack of research and knowledge encouraged the maintenance of negative attitudes. It is unknown whether changes in or maintenance of attitudes towards the standards is attributable to rationalization and reactance or if other experiences were influential. Thus, while rationalization and reactance are offered as one lens of interpretation, other explanations may be equally useful in understanding the study findings.

The next area in which study design may have introduced limitations is associated with the lack of experimental control, which may have introduced differences in the measurement and assessment of some constructs when compared to previous research. For example, the prior study of absoluteness that generated H2c (Laurin et al., 2012) 
manipulated perceptions of absoluteness through the use of stimuli. Given that the standards are not simply stimuli that can be varied but instead are inherently uniform across participants and have actual relevance for participants, it was not possible to manipulate their views related to absoluteness. Thus, the findings of this study may have provided slightly different information than what would have been obtained with generic stimuli that does not have actual impact. It is possible that perceptions of absoluteness were generally high because individuals had similar perceptions regarding the same stimuli (i.e., standards) and variation based on stimuli could not be assessed. While the lack of experimental design raises questions as to the comparability findings related to absoluteness, the use of qualitative methodology does promote accuracy in understanding the theoretical linkage between rationalization, reactance, and absoluteness (Fine \& Elsbach, 2000). Specifically, the role of absoluteness in differentiating these phenomena has only been assessed in one study. The current study provides information about the extent to which the association between these reactions holds when a real policy with personal implications is introduced. The current study suggests that absoluteness may not assist in differentiating these reactions when participant perceptions of absoluteness are considered rather than standardized stimuli. This finding is valuable as it may contribute to ensuring theory about the relationship between these constructs is practically relevant (Fine \& Elsbach, 2000). While this contribution is important and provides a vital step towards assessing these constructs in applied settings, it is important to reiterate that the current study does not directly assess rationalization and reactance and thus it is possible 
that the lack of support for the linkage between these constructs may be attributable to the way in which rationalization and reactance were approximated in the current study.

An additional limitation inherent in the study design is the lack of information regarding the directionality of social psychological experiences and compliance. It is possible that differences in the assessed social psychological constructs contributed to differences in compliance. Alternatively, it is possible that differences in compliance preceded any observed differences in the various social psychological constructs. The current study's use of a non-experimental design does not allow for speculation regarding the directionality of these effects. While this is the case, the segmented sample allowed for comparisons across the high and low compliance groups. This design feature provided information regarding the extent to which the social psychological processes included in the study differ across programs with varying levels of compliance. Additionally, given that this study examines reactions to an actual policy in the real world, it would have been exceedingly difficult, if not impossible, to design an experimental study that captured directionality without foregoing some of the ecological validity or real world relevance ingrained in the current study design.

Beyond limitations associated with study design, the analytic approach employed may have impacted the analysis of the interview data. Constructs were typically examined across programs in isolation rather than in relation to other constructs within the same individual. For example, perceptions of perceived control were not examined as they relate to absoluteness within each participant. Rather, the analyses focused on each construct across all participants and within person analyses were not consistently 
conduced for all combinations of constructs. This analysis choice was made to accommodate the research questions of interest, which were focused on the extent to which different responses and reactions occurred across participants. While this was generally the case, constructs including attitude change and absoluteness, as well as actual and perceived control, required a more detailed exploration into the constellation of these constructs in order to better understand how they manifest in relation to one another. It is possible that interesting themes and connections among constructs would emerge if thirteen unique within-subject analyses (i.e., case studies) were utilized. While it was outside the scope of the current project, future research could examine these interview transcripts as individual case studies in order to understand the consolation of each of these phenomenon and implementation processes within each participant.

Similarly, the analysis approach employed focused on the breadth of experiences and attitudes expressed by participants. This allowed for the acquisition of knowledge about the variety of reactions, responses, and experiences participants underwent during the process of implementing standards. While this method of analysis was valuable in identifying all experiences related to implementation, it did not account for the saliency of participant experiences. Specifically, the data was not analyzed to examine each participant's initial response to each question in relation to any relevant secondary information they provided. Thus, aside from the relative frequency of coded comments, it is unknown which comments were most salient to participants. Future research could examine reactions and responses to the implementation of a policy by focusing on 
saliency of perceptions in order to provide a more robust understanding of this phenomenon.

Finally, my own voice and biases as a researcher and person are likely inherent in this study (Stein \& Mankowski, 2004). Interpretation of qualitative data is a personal experience and I cannot remove myself from this process (Stein \& Mankowski, 2004). Several steps were taken to ensure that my point of view is not the only point of view expressed in the study. First, all aspects of the study, including the design, survey instrument, and interview questions, were created in collaboration with the Standards Advisory Committee. This allowed for multiple perspectives to be considered in the development of the study and made the study practically useful for the committee. Second, two trained research assistants assisted with any modifications to the predetermined codebook in order to ensure that any post-hoc changes were amenable to three separate individuals familiar with the interview transcripts and project. Third, the same two trained individuals conducted data coding and their responses were compared for inter-rater reliability in order to make certain the coders assigned codes consistently. Finally, within the Methods section of this dissertation I outlined my experiences and perspectives related to the Oregon BIP community and state standards in order to provide the reader with information regarding the perspective I brought to the study design, data collection, and analyses.

It is important to note that my experiences in the IPV community and understanding of the nuances and context related to BIP standards can also be considered strengths. My prolonged involvement in the Oregon IPV and standards communities 
bolsters the trustworthiness and credibility of the research findings. Specifically, I was able to design the study, conduct interviews, and complete analyses with contextual knowledge gained from prolonged exposure to these communities and opportunities to make observation across multiple contexts (e.g., Standards Advisory Committee meetings, local provider meetings, etc.). Additionally, my ability to maintain relationships with participants allowed member checking to validate the accuracy of the information shared during the interview process (Lincoln \& Guba, 1986; Merrick, 1999).

\section{Implications and Future Directions}

Theoretical implications. The unique premise of this study aimed to determine the extent to which policy has been implemented, along with program representatives' reactions and responses related to implementation. The study's mixed methods approach allowed several contributions to the BIP, social psychological, and social policy implementation literature.

Compliance with Oregon BIP standards. Phase One of this study updated previous work (Boal, 2010) to provide more current and comprehensive information about BIP practices and characteristics as they relate to a specific set of state standards. Previous work examined BIP practices in 2008, thus the current study investigated implementation after programs have had three additional years to adapt to standards. Other recent studies have examined the content of standards (Maiuro \& Eberle, 2008) or common program practices (Price \& Rosenbaum, 2009), but none looked at program practices and characteristics in the context of state standards. The information gained through Phase One of the current study is useful in better understanding how programs 
currently function in light of the standards. This phase of the study pinpointed components of standards that achieved high compliance, in addition to components that had low rates of compliance across all programs. This information is vital for understanding which components of the standards are actually implemented in programs across the state and which components have not yet been integrated into practice. Not only is this information useful for understanding implementation, it also serves as important preliminary information necessary to study the impact of standards on program efficacy.

It is important to note that the current study has examined implementation with the assumption that achieving compliance is sought after and positive. This assumption is consistent with the typical goals of policy implementation research (O'Toole, 1986; $2000 ; 2004)$. While this is typical of studies of policy implementation, compliance with Oregon BIP standards may not be wholly positive. Debate continues in the BIP research and practitioner communities regarding the extent to which standards are beneficial and based in research (Austin \& Dankwort, 1999; Holtzworth-Munroe, 2001). Given this debate and the uncertainty regarding the effectiveness of state standards, it is possible that compliance with standards does not necessarily translate into better outcomes for the men in these programs and their partners. For example, despite continued debate in the field (Holtzworth-Munroe, 2001; O’Leary, 2001; O’Leary et al., 1999) the state standards list couples counseling as a universally "inappropriate" intervention strategy and those who reported utilization of this strategy were deemed non-compliant with this component of the standards. While some scholars suggest couples counseling may be appropriate in 
some situations (see O'Leary, 2001 for a review), the standards do not allow for the use of this strategy at all. Thus, non-compliance with this component of standards could potentially reduce participant outcomes if couples counseling is in fact useful for some individuals. Given the lack of evidence for all components of standards, the extent to which compliance is positive should be critically considered when interpreting findings. Additionally, it is particularly interesting that Oregon state legislature calls for the use of state standards that are not based in evidence, due to the lack of evidence-based practice in the field, given another piece of legislation that is currently in practice.

Senate Bill 267 (SB 267) was passed in the Oregon State Senate in 2003. This bill requires the use of evidence-based practice in various contexts including mental health treatment, drug and alcohol treatment, and the prevention of re-offense for those in the criminal justice system. This bill was discussed in participant interviews as a concern given the lack of evidence for the practices of BIPs. Specifically, participants mentioned the conflict of state standards (SB 81) and SB 267 such that they were aware that BIP practices were not entirely evidence-based and were unsure whether following the standards was a violation of SB 267 . When thinking about how the seemingly divergent state standards and SB 267 can coincide there are several possible explanations. First, the standards were passed prior to the passage of SB 267. It is possible that because the standards were already in development, the guidance of SB 267 was not integrated into the standards. Second, SB 267 refers to specific agencies that must account for their use of evidence-based practices. While BIPs typically receive referrals from state agencies held to this law, such as the Department of Corrections and the Department of Human 
Services, they are not directly affiliated with these state agencies. It is possible that this disconnection from direct funding makes it more difficult to hold BIPs accountable to the requirements of SB 267. Third, it is plausible that despite the desire to ensure BIPs are based in evidence, the lack of evidence in the field makes it difficult to ensure this is being done. In the face of this the standards serve as a collection of agreed upon best practices from diverse members of the community collaborative response that can be utilize in lieu of evidence-based requirements.

Given the discrepancy between SB 81 and SB 267, it becomes important to consider the extent to which compliance with SB 81, or the state standards, is most important and should be prioritized. It is possible that while the current study focused on compliance with SB 81 and discussed how compliance could be increased, the lack of evidence for some components of the standards and inherent non-compliance with SB 267 may indicate that compliance with SB 81 is not necessarily positive. Thus, recommendations for increasing compliance discussed in the current study are presented under the assumption that compliance is desired with the understanding that there is little knowledge to determine whether compliance is actually useful for improving participant outcomes and victim safety. Despite this gap in knowledge, this study makes an important first step towards empirically examining the standards to determine whether the specific components, as well as the standards as a whole, are supported by research.

The current study provides the necessary foundation to conduct subsequent studies aimed at determining the impact of BIP standards on outcomes of interest including recidivism and victim safety. Studying the impact of standards on these 
outcomes would be flawed without a comprehensive understanding of the extent to which implementation has occurred (Derzon, Sale, Springer \& Brounstein, 2005; Durlak \& DuPre, 2008). Specifically, it is plausible that if non-significant or negative findings regarding the relationship between standards and outcomes were identified, it may be the result of non-compliance rather than a failure of the standards to achieve outcomes (Derzon et al., 2005; Durlak \& DuPre, 2008). Thus, having knowledge of implementation and compliance allows greater internal and external validity in studies of the impact of standards on recidivism and victim safety such that conclusions can be drawn with an accurate understanding of the extent to which standards were actually followed as intended (Durlak \& DuPre, 2008). With the information gained from the current study, future research should assess whether standards impact program effectiveness.

Future studies evaluating the effectiveness of state standards may utilize the findings from the current study in several ways. First, given the knowledge regarding variation in program practices in Oregon, it may be possible to pinpoint specific components of standards and evaluate the extent to which those components influence participant outcomes. It is possible that this could be accomplished within and between programs depending on the component of interest. Within program studies may be extremely useful because confounds such as location, staff, and curriculum would remain consistent, though this approach could only be used for components that vary in compliance within a given program. Specifically, because some programs reported being in compliance with some components of standards for a portion but not all groups, it may be possible to compare recidivism outcomes for individuals who attend groups that 
function in compliance with the component of standards and groups that do not. One useful example is the use of mixed gender co-facilitation. Survey findings indicate that $77 \%$ of programs have at least one group that utilizes mixed gender co-facilitation but only $56 \%$ of programs use this facilitation strategy for all groups. Thus, programs exist that comply with this component of standards in some but not all groups. This knowledge could be used to identify a program or programs with groups that do and do not have a mixed gender co-facilitation team in order to examine participant outcomes for those who experience this co-facilitation strategy and those who do not. This approach would account for contextual confounds and permit rigorous research design including random assignment to group. This evaluation strategy could be applied to additional components that vary within groups including group size and training of facilitators.

While within program studies to establish the effectiveness of some components of standards may be valuable, other components of standards pertain to the program as a whole and therefore cannot be studies within a single program. For these studies, data gathered in the current study may be useful in identifying programs that are common across most components of standards but differ in regards to the component of interest. It may be possible to identify programs that comply with the same components of standards except one component for which effectiveness can be examined. For example, data from Phase One could aid in identifying programs that comply with the same components of standards except in the case of completion requirements. If other aspects of compliance, as well as other program features (e.g., size and location) are similar, it may be possible to explore the influence of completion requirements on recidivism. While this approach is 
less controlled than the within program evaluations, it may shed light onto how discrete components influence recidivism across different programs.

While examining outcomes associated with differential compliance with specific components of standards is useful in establishing the extent to which each component is or is not supported by evidence, it is also possible to examine the impact of compliance as a whole. Data from the current study highlights the variance in compliance across programs. This information could be used to identify programs with similar contextual features but different levels of compliance and evaluate the extent to which recidivism differs for participants in each program. This process would provide insight into whether overall compliance with the standards is associated with positive outcomes, negative outcomes, or has no impact on participant outcomes. Explorations examining differences in outcomes within programs with variance in specific components, between programs with variance in specific components, and between programs with variance in overall compliance will provide much needed empirical evidence to inform the use and development of program practices and standards. Critics of standards (e.g., Austin \& Dankwort, 1999; Holtzworth-Munroe, 2001) and participants in the current study have noted that standards lack a solid evidence base. The current study provides the foundational knowledge to examine how differences in compliance with components of the standards impact outcomes. This in turn could lead to the establishment of evidencebased practices to guide BIP program characteristics and practices.

In addition to the importance of this study's findings generally, specific findings may also contribute to the BIP literature and BIP practice. This study demonstrated that 
programs tend to have implemented the majority of the components of standards assessed in the current study. However, there were several areas of non-compliance. Areas of noncompliance discussed below include those in which less than three-quarters of programs reported successful implementation. While these components are discussed, the argument can be made that all components of the standards should be improved upon, as perfect implementation across programs was almost non-existent. Alternatively, some may argue that non-compliance is indicative of program diversity and due to the lack of evidencebased practices in the field non-compliance may not be problematic. While both of these perspectives are valid, areas where non-compliance was greatest were examined in greater detail in order to better understand which components of standards were most problematic for implementation. The most salient areas of non-compliance tended to belong to four categories. These categories related to lack of compliance in areas requiring documentation, facilitator training, collaborative efforts, and in areas that may be especially important for victim safety.

The first general category of non-compliance, where more than one-quarter of participants had not achieved implementation, was related to difficulties maintaining and distributing documentation. A substantial number of programs do not have all written policies and procedures called for by the standards. Similarly, a sizable number of programs do not keep records of program completion after intake. Additionally, the number of programs who distribute all types of information recommended by the standards to victims and partners was limited. Each of these areas of non-compliance is related to tasks that require documentation and development of materials. There are 
several possible reasons for the lack of required documentation. First, it is possible that the lower compliance observed for these components of the standards is related to the time required to develop these materials within organizations that have limited time for additional tasks beyond the day to day operation of the program. One way to address this burden and encourage greater documentation would be to provide example and template documents from which providers could model their materials. A second possibility for non-compliance related to documentation is that programs are unaware that written documentation is necessary. Discussion with one interview participant revealed that completion of the BIP Survey alerted them to the fact that these materials were important and lead to the subsequent development of written materials for this program. Thus, education and outreach to programs to inform them more clearly about the requirements of the standards may increase implementation in this arena.

The second general category of non-compliance is related to requirements for collaboration and community involvement. Theoretically, prevention and intervention will be more successful and less fragmented if the entire community is held responsible for holding perpetrators accountable and ensuring survivor safety, rather than individual agencies alone (Allen, Watt, \& Hess, 2008; Klevens, Baker, Shelley \& Ingram, 2008; Shepard, Falk \& Elliot, 2002). Ensuring collaboration occurs is important as advocates for its use have indicated that a collaborative approach has benefits that reach beyond reducing abusive behavior and criminal recidivism, including increasing access to services for survivors and creating systems level change in the judicial response to IPV (Allen et al., 2013; Klevens et al., 2008). 
While reports of collaboration with community partners were high, they were less so for more specific and relationship-intensive aspects of compliance. For example, while participants reported general collaboration with the criminal justice system and victim advocacy agencies, when asked about specific qualities of the collaborative relationship, such as having the victim advocacy agency review their policies and procedures, compliance was lower. More specifically, programs reported lower compliance with the requirements that a victim advocacy agency must review program policies and procedures, outcome and attendance of participants must be reported to a liaison in the criminal justice system, programs must participate in BIP organizations to the extent possible, and programs must engage in outreach to the community to the extent possible. Each of these components requires more than just having contact with a community partner. Instead, these components call for active participation and engagement. Specifically, programs are expected to have liaisons and communicate with criminal justice and victim advocacy agencies, attend meetings with these agencies, collaborate with other BIP providers, and perform outreach to the community. Thus, to increase compliance programs may need further guidance about how to maximize the relationships they currently have in order to allow for more intensive collaboration. Further, some programs may need to be connected to community partners. For instance, it may be more difficult for programs that are geographically isolated to participate in a BIP organization in a conventional way. Those located in rural areas may have to travel long distances to attend meetings, incurring both monetary and time costs for travel. Additionally, while programs located close to meeting locations can take minimal time 
away from their program responsibilities to attend meetings, those farther away would likely have to take the entire day or days off and may not be able to provide services to clients because of this absence. Increasing access to community partners though technology such as online forums, options to call in to meetings, or webcasts of meetings, may provide more opportunities for meaningful involvement. Interview participants suggested that a statewide association of BIP providers or even a statewide e-mail listserv would be a valuable resource to enable more extensive and meaningful collaboration. While remote collaboration is different than in-person collaborative efforts, investigations of the success of DV councils in achieving council goals include qualities such as climate, structure, and membership as gauges of successful collaboration (Allen, 2005; 2006). Specifically, councils that are inclusive of diverse perspectives and active are rated more positively and are more effective in achieving council goals (Allen, 2005). Thus, even if they have to participate remotely, including rural providers in collaborative efforts may broaden the views of the council and allow for a larger number of members to actively work towards collaborative goals.

Interview participants raised two additional explanations for the lack of collaboration with community partners: problematic relationships among collaborative partners, and the community partner's lack of time and resources to achieve collaboration. Interview participants noted that collaboration is difficult when relationships between the BIP and available collaborative partners are not ideal. Collaboration can only occur when all partners are willing and able to participate in the collaborative relationship, an issue that may be especially important to address in the 
field of IPV. Scholars have discussed the difficulties inherent in collaboration in situations of inter-group conflict and have noted that IPV is one area in particular that is marked with a history of difficulties (Allen, 2006; Foster-Fishman, Perkins \& Davidson, 1997). Active involvement of partners from diverse aspects of the response to IPV is necessary for the success of collaborative councils (Allen, 2006). Thus, addressing longstanding tensions among collaborative partners is vital for the collaborative relationship to yield desired outcomes. Given the longstanding tensions in the IPV community, alleviating this problem may require both time and honest conversation among community partners to identify sources of tension and work towards identifying mutually agreed upon goals for collaborative efforts. Further, interview participants noted that collaboration was problematic when collaborative partners did not have time or resources to devote to progressing the collaborative relationship. For example, interview participants described that other agencies were just barely getting by, making it difficult to spend time and resources to improve collaboration. Identifying ways that collaboration could be mutually beneficial, such as sharing of resources, referral of clients, or reciprocal trainings, may free up resources and time to encourage collaboration.

The third category of lower compliance was related to the training of group facilitators. Slightly more than half of the programs indicated all facilitators had completed the required victim advocacy training and had completed the required BIP training. This indicates that a substantial proportion of programs are functioning without all staff having achieved the training deemed necessary by the state standards. While this is the case, within programs approximately three-quarters of facilitators had achieved the 
required trainings. Together this suggests that most facilitators have fulfilled the training requirements, though nearly half of programs do not have all facilitators who have reached the training criteria. It is possible that some facilitators within these programs are new to the field of batterer intervention and have not yet had the opportunity to participate in these trainings. Alternatively, it is possible that some facilitators have neglected to complete the trainings despite having served as a facilitator for a prolonged period of time. The interview portion of the study suggested that non-compliance with the training requirements is often due to structural factors including training location, financial resources, and time. Interview participants described difficulties inherent in attaining training when trainings were held at a great distance from their program location, trainings were expensive to attend, and the size of the program could not compensate for staff time away. For example, a representative from a small, geographically isolated program may have to travel a great distance, incur costs for travel above those that exist for the training, and experience a loss of income from having to cancel groups that the facilitator leads during their time away. This likely makes achieving compliance with the training requirements much more difficult than what is experienced for larger programs in metropolitan areas.

Whether lack of training is due to the newness of the employee or barriers to attaining training, ensuring that those working with group participants are knowledgeable about batterer intervention strategies thought to be best practice and is required by most state standards (Austin \& Dankwort, 1999; Maiuro \& Eberle, 2008). Increasing compliance with the training components of standards for all facilitators could be 
accomplished in several ways. First, as was suggested related to collaboration, trainings provided online or over the telephone may be useful for enabling programs with fewer resources or located remotely to access training opportunities. Second, the state could organize and hold trainings periodically that rotate in location and include rural areas. This would allow for face-to-face training and relationship building, while easing the burden of extensive travel for some programs. Third, a consortium of those who provide victims advocacy or BIP trainings could be developed in order to make it easier for programs seeking training to request trainings be held. This would allow programs to notify the consortium when new staff members are hired so that trainers are aware that trainings are needed. This could be useful in ensuring trainings are held when there is a need rather than at an arbitrary time. Alternatively, the consortium could compile a list of all trainings planned for the upcoming year so that programs are aware of all opportunities in all locations and have time necessary to plan, both logistically and financially, for the trainings.

The fourth category of lower compliance included the use of intervention techniques specified in the standards as "inappropriate". More than $25 \%$ of programs reported having contact with victims for reasons prohibited by the standards. A minority of programs endorsed causes of battering and intervention strategies prohibited by the standards. While these prohibited techniques were only observed in a minority of programs, they may have important implications. First, the requirements for victim contact were developed to maximize victim safety. If programs are not adhering to these regulations, they may be placing victims at greater risk for further victimization. For 
example, the standards outline when contact is "appropriate" (e.g., provide information about participant attendance, discharge, or referral to resources) in order to reduce the risk that contact with victims will make the victim feel pressured to disclose information about the client. If victims were pressured to disclose information and the client knew or suspected this was the case, this may place the victim in danger. The requirement that these policies and procedures be reviewed by a victim advocacy agency is intended as a further protection to ensure that victim contact does not negatively impact victims.

Second, the endorsement of prohibited causes of battering and intervention techniques highlights possible philosophical differences between the standards and some providers. Again, the standards are rooted in the premise that utilization of techniques deemed "appropriate" in the standards will promote change and increase victim safety. When providers are adhering to techniques such as encouraging victim or partner disclosure of information or participation, the victim or partner may be placed at greater risk. Because the use of these prohibited strategies is not the result of structural barriers to compliance with state standards, such as time or money, but is instead likely rooted in philosophical differences between the standards and providers, changing the use of these strategies may be especially challenging. For example, if providers require victim or partner disclosure because they fundamentally believe the information is vital for promoting behavior change, providing additional resources may not change this philosophical view of IPV. To change prohibited practices, such as the requirement for victim or partner disclosure, a different approach would be necessary. Rather than providing relationships or resources, changing these practices may require tactics such as 
education to convey the rationale behind the prohibition of these strategies or increased penalties for those who utilize these strategies. Education about the rationale and empirical support for these components of the standards may be valuable in addressing discrepancies in practice and philosophy for these programs. Interview participants indicated that trainings provided by the state would be helpful to ensure greater comprehension of the standards and why the various components of standards are important. It is possible that a yearly state training for all providers would be immensely beneficial in not only outlining the specifications of the standards, but also providing contextual knowledge to help providers understand the rationale of each component and why adherence is important.

In addition to areas of non-compliance, there are two components of the standards that were assessed and are interesting to think about in greater detail. The first component that may be useful to examine further is program length. Previous research has demonstrated that shifts in average program length for BIPs in Oregon have coincided with changes to the state standards (Boal, 2010). Contrary to what has been found previously, the current study determined that programs require an average of 44 weekly sessions in order to complete the program, four weeks shy of the 48 weeks required by the standards. The most recent assessments of state standards and program practices nationwide found that most state standards require 24 to 26 weeks of intervention (Maiuro \& Eberle, 2008) and on average most programs in the U.S. are approximately 31 weeks in length (Price \& Rosenbaum, 2009). Thus, while Oregon programs were on average shorter than what is expected based on the standards, their length exceeds what is 
typically expected by standards and achieved by programs nationally. Nonetheless, the program length is a decrease from the prior assessment of BIP practices in Oregon, which found that most programs required approximately 50 weeks of intervention (Boal, 2010). This indicates that programs have on average become shorter since 2008. There are several possible explanations for this change. One explanation is that during the time of the survey, the Standards Advisory Committee was in the midst of making changes to the length requirement of the standards. While the Standards Advisory Committee has the ability to suggest revisions and refinements to the standards as needed, a major change had not been made since they were originally created. Thus, this was a new experience for providers. The updated standards, which were publically posted on September $1^{\text {st }}$, 2013 (approximately 9 months after the 2011 BIP Survey closed), now require that programs utilize a minimum of 36 sessions and submit a summary report justifying the need for additional sessions if they believe additional sessions are warranted. This raises the possibility that some programs were aware that required program length would be shortened and had begun shifting program practices to account for the upcoming change. Conversation during the qualitative interviews revealed another potential explanation: local standards sometimes mandate shorter program length. Specifically, a number of participants indicated that some areas in Oregon utilize local standards that diverged from the state standards in terms of program length. It is possible that the local standards have impacted the average length of programs as some providers may be expected to require shorter programs. These local forces may explain why the average program length decreased since 2008 such that it dipped below what is expected by the standards. 
While differences between local and state standards were described as problematic for some participants as it caused confusion in which standards should be followed, it may also provide an opportunity for further research examining program length. Given that some areas require different program lengths, it may be possible to examine outcomes for participants who take part in programs with different lengths, though there would be many confounds inherent to a study comparing different programs in different areas that may be difficult to navigate. Despite the difficulties in examining outcomes related to length, one area of inquiry that would be less fraught with methodological issues would be the exploration of how length impacts the materials and content covered by programs. This would provide novel insight to determine what curriculum and programmatic differences coincide with shortened program length and allow speculation into how that may impact the education received by participants. Thus, the findings of this study may situate future work that aims to better understand the meaning of changes to program length for service delivery.

The findings on mixed-gender co-facilitation also should be compared to national practices and standards. The last assessment of program practices nationwide (Price \& Rosenbaum, 2009) found that one-third of programs use a mixed-gender co-facilitation approach in the majority of their groups. The current study found that $56 \%$ of the programs in Oregon report utilizing this strategy for all groups, which surpasses typical practice nationally. The fact that most BIPs in Oregon exceed national norms related to mixed-gender co-facilitation is noteworthy given the theoretical association between mixed-gender modeling of appropriate behavior. Theoretically, this model of co- 
facilitation is expected to be beneficial because it provides an opportunity for modeling which may in turn promote behavior change (Bandura, 1974; Gist, Schwoerer \& Roser, 1989; Sarason \& Ganzer, 1973). Specifically, this facilitation approach serves as a model of healthy male-female relationships and provides an opportunity for the men to interact with women in an appropriate manner (Adams \& Cayouette, 2002; La Violette, 2001; ODOJ, 2009). Beyond the theoretical merit of mixed-gender co-facilitation, one recent study (Roy, Lindsay \& Dallaire, 2012) found that men in BIPs reported mixed-gender cofacilitation was useful in relation to the discussion of violence, support provided by facilitators, and in managing group dynamics. Thus, the relatively common use of mixedgender co-facilitation among BIPs in Oregon aligns with current best practices for group facilitation in BIPs. While the reason behind the more widespread use of mixed-gender co-facilitation in the state of Oregon is unknown, it is possible that the inclusion of this recommendation in state standards promotes the use of this strategy.

This information is useful when considering how the effectiveness of mixedgender co-facilitation can be evaluated. While the majority of programs use mixedgender co-facilitation in all groups, nearly one-third indicated they utilize mixed-gender co-facilitation in some but not all groups. These programs may provide an optimal environment to examine the impact of different facilitation models. Specifically, if all other program features are identical and the only distinction between groups is facilitation style, it may be possible to randomly assign individuals to different groups with differing facilitation styles and examine potential differences in partner-reported re-offense or recidivism detected by the criminal justice system. Building an improved body of 
empirical evidence to situate the standards may in turn positively impact perceptions of legitimacy towards the standards.

Social psychology. The current study provided an opportunity to evaluate social psychological phenomena that may underlie BIP representatives' responses to state standards. This study aimed to determine the extent to which the phenomena of actual control, perceived control, attitude change, absoluteness, and legitimacy were experienced in the sample of interview participants. Developing a better understanding of these phenomena is useful in determining how these theoretical constructs manifest during the introduction of a new policy in the real world. Further, this study provides insight regarding opportunities to build capacity and encourage greater implementation by addressing experiences that may inhibit greater compliance.

Actual control. Actual control refers to the extent to which individuals have control over their behaviors (Ajzen \& Madden, 1986). Actual control is important to consider because having actual control typically indicates that the individual has access to resources and greater ability to reach a desired outcome such as implementation of standards (Ajzen \& Madden, 1986). Additionally, having actual control in the form of shared decision-making power is associated with better implementation (Durlak \& DuPre, 2008). Given that experiences of actual control, including shared decision-making power, are expected to be associated with having better access to resources, ability to achieve desired outcomes, and better implementation (Ajzen \& Madden, 1986; Durlak \& DuPre, 2008), it was expected that high and low compliance program participants would report different experiences related to actual control. 
It is worthwhile to consider how actual control manifested in the current study and why this may be associated with compliance. Participants were considered to have actual control when they played an active role in informing the content of the standards, or were knowledgeable about the standards creation process or those involved in the process. There are several components of compliance that may go hand in hand with actual control as it was operationalized. Those who report participating on, providing feedback to, or knowing members of the Standards Advisory Committee are likely those who have relationships with others in the BIP community. Specifically, they must be well connected enough to be aware of who developed the standards and how they were developed, indicating they have ties to the IPV community. This is immensely important as community collaboration is an explicit component of the standards and having these connections to the Standards Advisory Committee may indicate that the provider is better connected in general. Additionally, when participants were asked to describe enablers, barriers, and support need to achieve compliance, positive and information sharing relationships with others in the BIP community were described as enablers and support needed, while negative or lack of relationships were described as barriers. Those who participated on the Standards Advisory Committee by definition are an active part of the IPV community as they are required to have working relationships with and participate in regular meetings with different members of the community collaborative response. Additionally, participants who indicated that they felt isolated from the BIP community were also less informed about what the standards included, indicating there is an opportunity for education among low compliance programs. Thus, it may be the case that 
lower compliance programs have fewer connections to enable active and positive relationships, which may lead to isolation and lower comprehension of the standards. This logic indicates that increasing connections among those in the IPV community may increase the sense of actual control among providers, which may in turn increase compliance.

The hypothesis that actual control would differ across high and low compliance programs was not supported inferentially. While this is the case, it is important to note that the small sample size likely impacted the current study's ability to detect the large effect $(d=1.21)$ that was identified. Further research with a larger sample size is needed to more comprehensively identify possibly statistically reliable differences in actual control among high and low compliance BIPs.

The specific content of the interview responses was helpful in more precisely understanding the experiences of actual control across high and low compliance programs. These findings provide some support for the theoretical connection between actual control and achievement of outcomes (i.e., compliance) such that compared to low compliance programs, high compliance programs spoke more deeply and comprehensively about experiences consistent with having actual control over the standards (e.g., serving on or providing direct feedback to the Standards Advisory Committee). This finding is consistent with previous work suggesting that having decision making power influences implementation (Durlak \& DuPre, 2008) and points to the importance of offering opportunities for provider involvement in the standards development and refinement process. One way that this could be achieved is through 
allowing more providers on the Standards Advisory Committee. While this is an option, the current committee is composed of diverse individuals related to IPV intervention in order to ensure multiple perspectives are accounted for. Though increasing the number of providers may increase their experiences of actual control, this may negatively impact the diverse composition of the Standards Advisory Committee. An alternative option would be to increase transparency of the standards creation process and access to members of the Standards Advisory Committee in case questions or concerns arise. Increasing transparency and access would ensure that providers had a clear understanding of the process by which questions or concerns can be raised and would enable them to take action if necessary. This option could increase actual control while maintaining the diverse opinions of the Standards Advisory Committee.

Perceived control. In addition to actual control, perceived control was also considered. The current study utilized the conceptualization of perceived control in the sociopolitical context offered by Zimmerman and Zahnsier (1991). This aspect of perceived control is centered on the extent to which an individual believes they can successfully navigate and impact policy decisions and political systems (Paulhus, 1983; Zimmerman \& Zahniser, 1991). Given this conceptualization, perceived control was operationalized to include perceptions regarding each participant's ability to meaningfully contribute to the content of the standards and/or the Standards Advisory Committee. When asked about perceived control over the standards it was evident that for most participants, both the presence and absence of perceived control were described. This is consistent with prior work, as scales assessing sociopolitical control allow 
participants to identify the areas in which they perceive control and do not perceive control, indicating it is possible to experience a mixture of perceptions related to different aspects of control over a policy (e.g., Paulhus \& Van Selst, 1990; Zimmerman \& Zahniser, 1991).

Prior research suggested that increased perceived control is associated with increased action towards desired outcomes, which equates to compliance in the current study (Holden et al., 2004; Itzaky \& York, 2000; Ohmer, 2008). Thus, it was hypothesized that increased perceived control would be associated with greater compliance. This hypothesis was not supported, though, as was the case with actual control, a large effect size $(d=.93)$ was observed. While this is the case, the specific content of interview responses was useful in explaining the nuances of perceived control across participants.

Those who reported primarily perceiving control over the standards indicated they knew who to talk to when questions or concerns about the standards arose and were confident their voice would be heard. Most of the high perceived control participants had these views because of prior experience but some felt they would be able to impact the standards simply because they are confident in their ability to make change, if desired. This points to two facets of perceived control: perceived control that is based on previous experience and perceived control that is based on internal attributes of the program representatives. This is consistent with prior examinations of perceived control that suggest that personality, cognitive, and motivational factors all influence perceived control (Zimmerman \& Zahnsier, 1991). Additionally, studies have demonstrated that 
self-efficacy (i.e., perceived ability to make necessary changes) for implementation is associated with better implementation (Dufrene, Noell, Giberson \& Duhon, 2005; Durlak $\&$ DuPre, 2008; Sterling-Turner, Watson \& Moore, 2002). While this is the case, effort to impact program directors' internal attributes in order to increase their perceived control with the hope of increasing program compliance may be especially difficult and unrealistic in this context. This would likely require one-on-one interactions to identify the needs of each individual program director and a tailored attempt to increase internal attributes related to control. This process could potentially be beneficial but is likely outside the scope of the role of the Standards Advisory Committee. Alternatively, it may be more feasible to provide opportunities to increase experience-based aspects of perceived control. Increasing perceived control through greater experiences related to control could be accomplished simply by conducting outreach to ensure providers are aware of the Standards Advisory Committee and have up to date contact information in case they have a concern. Actions as simple as outreach to program directors may provide an increased sense that if a change were desired, the individual would know where to go to advocate for that change.

Actual and perceived control. Studies examining the connection between actual and perceived control as related to policy have indicated that higher actual control is associated with higher perceived control (Becker, Israel, Schulz, Parker \& Klem, 2002; Kieffer, 1984; Schulz, Israel, Zimmerman \& Checkoway, 1994). The findings of this study did not support an association between actual control and perceived control over the standards when tested inferentially. Further research with a larger sample would be 
valuable to determine if the lack of inferential support is an artifact of the extremely small sample size or if this relationship truly does not exist in this context. While a statistically reliable association was not detected, the pattern of responses suggested that those who described more comprehensive experiences of having actual control also perceived having a greater degree of control moving forward.

The theoretical link between actual and perceived control is valuable due to its potential utility in improving compliance. Specifically, while perceived control was associated with outcomes in previous studies (Holden et al., 2004; Itzaky \& York, 2000; Ohmer, 2008), it may not be necessary to influence perceived control directly when trying to achieve an outcome of interest such as compliance. Instead, focusing on improving individuals' sense of actual control may be most useful. Specifically, increasing an individual's internal confidence could influence perceived control or by increasing the number of successful experiences of control they have to draw from and form perceptions about control. Addressing perceptions of control that are rooted in internal confidence would not likely occur in the context of encouraging compliance due to the personalized and personal investment necessary to make such changes. Instead, it is likely most beneficial to focus on increasing the number of successful experiences of control that the individual can base perceptions upon. Thus, having meaningful actual control experiences becomes vital.

Research suggests that increasing actual control will contribute to an increase in perceived control over the standards moving forward by allowing for a better sense of who to talk to in order to impact the standards. Additionally, increasingly meaningful 
experiences of actual control may provide opportunities for positive experiences with those involved with the standards. An increase in actual control could impact compliance directly, as well as indirectly by boosting perceived control. Future studies could further assess the connection between actual control, perceived control, and compliance in a larger sample of BIP representatives in order to determine if the large effect size observed translates into a statistically reliable difference in compliance among those with high and low actual control and high and low perceived control, as well as perceived control among those with high and low actual control. Additionally, it may be valuable to assess how these constructs are impacted by increased opportunities to learn about or provide input for the standards in order to determine if the theoretical suggestion of increasing opportunities to interact with the standards and the Committee are useful for increasing compliance.

Attitude change and absoluteness. In addition to the theoretical questions raised by this study related to the actual and perceived control literature, this study also provokes questions regarding the experiences of changing or maintaining negative attitudes. One construct that may aid in explaining attitude change is that of rationalization. The construct of rationalization stems from the cognitive dissonance literature (e.g., Festinger, 1957) and is focused on explaining one way that individuals respond to a situation where their beliefs and behaviors are misaligned. While studies have investigated this phenomenon as it relates to voluntary behaviors, it can also be applied in situations where behavior change is not voluntary, such as compliance with a mandated policy (Gilbert et al., 1998). In these cases, individuals are motivated to change 
their negative cognitions about the policy at hand in order to remove discomfort associated with making changes consistent with the policy's requirements. Thus, in the current study those who initial viewed the standards as negative but shifted their views over time to perceive the standards as primarily positive were examined, with one possible explanation for this shift being the process of rationalization. Alternatively, reactance stems from the notion that when freedoms are limited, individuals are motivated to maintain their freedoms and tend to view whatever or whoever is attempting to limit their freedoms more negatively (Brehm, 1966). Again, this phenomenon has been assessed in the context of the introduction of a policy (e.g., Erceg-Hurn \& Steed, 2011; Mann, 2010). These studies suggest that when freedoms are limited, participants may experience resistance and report greater misalignment with the goals of the policy (ErcegHurn \& Steed, 2011; Mann, 2010). Thus, those who initially viewed the standards as negative and currently maintain their negative views were examined, with one possible explanation for this attitude consistency being reactance.

It is important to stress that studies of both rationalization and reactance are inherently flawed when studied retrospectively. This is due to the necessity for information about perceptions and attitudes before and after the introduction of some freedom limiting intervention. To best assess rationalization and reactance information regarding perceptions in real time, rather than retrospective recollections, is preferred. While this is the case, studies have attempted to understand these phenomena by exploring reported attitudes and beliefs before and after the introduction of stimuli that vary in the extent to which they limit freedoms (e.g., Beasley \& Joslyn, 2001; Dillard \& 
Shen, 2005; Kay et al., 2002). Thus, while not perfect, the current operationalization of these constructs allows for the acquisition of cursory knowledge about whether and how participants change their views after the introduction of standards. While this is the case, it remains unknown whether any attitude change or lack of change is due to rationalization and reactance or other experiences (e.g., greater familiarity, education, etc.).

There is limited research exploring the experiences of rationalization and reactance for required behaviors. This may be due to the difficulties associated with assessment of these experiences in situations where behavior change is actually required. The current study experienced difficulties in measurement of rationalization and reactance due to the reliance on retrospective accounts of initial attitudes towards the standards. In the face of this challenge, participant reports of initial responses and current responses were used to examine change or maintenance of negative attitudes, and this served as a rough proxy for the experiences of rationalization and reactance. Though the measurement of these constructs was not ideal, this study provides descriptive, qualitative information that can be used to guide further and more standardized examination of these processes when change is not a choice. The limited research examining rationalization in the context of non-voluntary behaviors, such as the need to conform to a new policy, suggests that when individuals experience cognitive dissonance they are motivated to shift their attitudes to reduce dissonance (Beasley \& Joslyn, 2001; Granberg \& Brent, 1983; Kay et al., 2002). This suggests that individuals who initially view the standards as primarily negative but are expected to comply with the standards despite negative initial 
perceptions may change their views to view the standards as primarily positive, thus having a reaction that could possibly be attributable to rationalization. Further, it was hypothesized that participants who changed his or her initial negative attitudes (i.e., a proxy for rationalization) would include representatives from high compliance programs as the behavior of complying with standards and current positive view of the standards would be aligned. This hypothesis was supported as nearly every participant who reported changing his or her initial negative attitudes represented a high compliance program.

Determining the extent to which negative attitudes shifted to positive attitudes, which could possibly be attributed to rationalization, occurred within the sample and the prevalence of high compliance programs among those who shifted negative attitudes is valuable for understanding responses to an involuntary change required by the introduction of policy, an area that has received relatively little attention in the empirical literature. Even when a policy is initially met with fear or distrust, it may be possible to overcome these initial negative responses and encourage greater acceptance of the policy. Further, those who are able to overcome their negative initial beliefs represent programs that were more successful in integrating the policy into their program practices. However, it is unknown whether a shift in views preceded changes in program practices or if changes in program practices preceded a shift in views. Determining whether programs' ability to change practices or the shifting of attitudes was the first step towards greater compliance would help identify the best point of intervention to promote shifting attitudes to be more positive and compliance. Specifically, it would disentangle whether 
providing support for structural changes that would directly impact program practice is most important or if further education and outreach to change perceptions would be most valuable.

In addition to shifting negative attitudes, the maintenance of negative attitudes, which could possibly be attributed to reactance, was also assessed. Though not ideal, using maintenance of negative attitudes as a proxy for reactance was useful in examining the findings from the current study in the context of the reactance literature. Previous research and theory suggests that reactance occurs when individuals attempt to maintain threatened freedoms, such as one threatened by the introduction of policy, and continue to view the threat to freedoms negatively (Brehm, 1966; Dillard \& Shen, 2005; ErcegHurn \& Steed, 2011; Mann, 2010). Given that resisting changes is a feature of reactance, it was hypothesized that those who maintained negative attitudes (i.e., possibly experienced reactance) would represent low compliance programs. This prediction was supported as every participant who initially had negative views towards the standards and maintained their views was from a low compliance program. The findings from the current study lend further support to the one possible consequence of the introduction of a new policy, the lack of policy implementation (i.e., non-compliance), which may occur when individuals maintain negative attitudes towards the introduction of a policy.

Given that some individuals maintain their negative views of the standards and are also those who are most out of compliance with standards, it is useful to identify their views more precisely. As was mentioned, these individuals tended to have negative views about the specific components of the standards that persisted over time. These 
perceptions represent a different outlook than what was observed in participants who were able to shift their negative attitudes into positive attitudes. Those who were able to shift attitudes made more comments indicating a sense of general fear and ambiguity initially, rather than just discussing only the components of the standards with which they disagreed. This suggests more targeted outreach may increase compliance to address concerns of those who have maintained negative attitudes. An additional tool for addressing the maintenance of negative attitudes that may be valuable to consider is motivational interviewing. This approach would involve discussions with program directors or program representatives to provide support and encourage them identify their own motivations to make program changes based on standards (Hettema, Steele, \& Miller, 2005). Motivational interviewing has been found to be useful when individuals are resistant to change (Hettema et al., 2005). If monitoring of standards becomes more common in the future, it may be possible to integrate motivational interviewing into the monitoring process. For instance, if a representative from the state or Standards Advisory Committee communicates with or visits each program, they may incorporate tools from motivational interviewing to encourage greater compliance. Future research could assess the effect of education regarding specific program components and the rationale for those components, as well as the use of motivational interviewing, in promoting greater compliance. This education and outreach could be beneficial to address specific concerns of those who have maintained negative attitudes towards the standards, but also be useful to increase communication and build connections with other providers and other members of the community collaborative response, which may impact actual and 
perceived control. Further, if education and outreach can explain why the components of the standards were selected and connect them to evidence-based practice or best practice, it is possible that the perceived legitimacy of the standards may be increased as well.

Taken together, it appears that some participants were able to shift their negative attitudes while others maintained negative attitudes and whether or not shifts were made may help explain compliance with standards. Initial perceptions were varied in the two groups with those who shifted their negative attitudes providing more comments indicating a global sense of fear or uncertainty, while those who maintained their negative attitudes described more specific aspects or characteristics of the standards with which they disagreed. Thus, it is possible that global concerns that could be addressed through education or greater familiarity with the standards occurred over time without explicit effort by the Standards Advisory Committee. This may have allowed individuals to construct an explanation for their experience of dissonance such that their initial discomfort was due to unfamiliarity rather than actual negative views of the standards, which in turn could have contributed to a lessening of cognitive dissonance and greater acceptance of the standards. This explanation coincides with other studies of the response to the introduction of unavoidable changes, such as elections or policy changes, which indicate that when personal freedoms are limited by policy, individuals are motivated to change their beliefs to make the policy more attractive (Beasley \& Joslyn, 2001; Granberg \& Brent, 1983; Kay et al., 2003). Conversely, as the vast majority of the components of standards have persisted since their creation, changing negative perceptions related to specific components may be more difficult. If the components of 
the standards that negative attitude maintenance participants disagreed with were initially viewed as extremely important, the theory of reactance suggests that these individuals would be motivated to maintain those limited freedoms and thus negative perceptions have persisted (Brehm, 1966). While the type of comments related to initial perceptions provided by participants (i.e., global versus specific) may provide insight into the different responses to the introduction of a new policy, another possible factor for differentiating these experiences, absoluteness, has been recently suggested in the literature.

Absoluteness in the context of policy introduction is the extent to which the enactment of a policy is certain (Laurin et al., 2012). The only study that has assessed the role of absoluteness in helping explain these reactions found that absoluteness is useful to distinguish those that experience rationalization versus reactance after the introduction of a new policy (Laurin et al., 2012). Thus, the current study hypothesized that the findings of Laurin and colleagues (2012) would hold in the current sample. While the current study did not directly assess rationalization and reactance, negative attitude change (i.e., rationalization) and maintenance (i.e., reactance) were used as proxies to examine the role in differentiating responses based on absoluteness. In contrast to the findings of Laurin and colleagues (2012), in this study, absoluteness varied substantially within both the negative attitude change and maintenance groups.

There are several possible explanations for the lack of support in the data for this hypothesis. First, the previous study (Laurin et al., 2012) examined absoluteness as an experimental condition rather than in relation to participants own perceptions. In the 
current situation all participants have received the same set of standards and the extent to which absoluteness is observed is the result of the participants own interpretation, understanding, and contextual experiences. This is quite different than all participants receiving standardized materials that vary in terms of absoluteness. Specifically, in the current study, absoluteness is conceptualized as internal to the participant rather than an external feature of the experimental stimuli. This may have resulted in differences in the ability to compare absoluteness in the negative attitude change and maintenance groups. As the relationship established by Laurin and colleagues (2012) has yet to be replicated, more research is needed to determine whether the lack of support for the current study is due to differences in measurement (i.e., quantitative versus qualitative; use of attitude change proxy rather than measurement of rationalization and reactance) and context (laboratory-based versus field-based) or due to the lack of a robust relationship between these constructs.

Legitimacy. Finally, the study assessed whether perceptions of legitimacy might help explain implementation of the standards. The definition of legitimacy used to guide the current study focused on the extent to which those in power are believed to make fair and appropriate decisions (Tyler, 2006). Obtaining legitimacy is important because individuals viewed as legitimate have greater latitude to make decisions and their decisions are viewed more favorably (Tyler, 2006). Additionally, compared to other power tactics (e.g., coercive power or reward power) legitimacy may be a more effective and less costly means of increasing implementation or obtaining compliance (Tyler, 2006; Tyler \& Ho, 2002; Stevenson et al., 2002; Wallner, 2008). When attempting to 
understand the extent to which a power authority and policy are viewed as legitimate, three components of legitimacy should be considered. These include procedural justice, social norms, and policy logic. Procedural justice includes the extent to which individuals believe that those making decisions are doing so fairly (Thibaut \& Walker, 1975). A synthesis of available studies showed that those who appear to display procedural justice are viewed as more legitimate (Tyler, 2006). As is described in the legitimacy literature, social norms include the extent to which others impacted by the policy view the policy as positive or negative. Thus, valence of norms is immensely important in relation to this conceptualization. Norms are transmitted through interactions and provide information about what is normally done, what should be done, and what others view as appropriate (Cialdini \& Trost, 1998). Thus, norms can be based observations of the behavior of others. Research related to legitimacy of authority broadly has demonstrated that those who report positive social norms also report increased legitimacy towards the policy and the policy authority at hand (Tyler, 2006; Zelditch, 2001; Zelditch \& Walker, 2000). Finally, policy logic refers to the extent to which the policy at hand is based in evidence and best practice in the field. Studies indicate that policies viewed to be high in policy logic are also perceived as more legitimate (Stryker, 1994; Wallner, 2008). Thus, the current operationalization of legitimacy included these three components. Each component of legitimacy was captured using a pair of two codes to represent presence or absence of procedural justice, positive or negative norms, and presence or absence of policy logic. 
It is evident that the vast majority of comments related to procedural justice raised questions about the fairness of the Standards Advisory Committee and standards creation process. The lack of procedural justice had a profound impact on overall ratings of legitimacy. Statements indicating a lack of procedural justice highlighted the lack of representation for diverse fields, geographic areas, and viewpoints on the Standards Advisory Committee. Comments relating to the lack of diversity were varied but many centered on the lack of input from individuals from: ethnic or racial minority groups; lesbian, gay, bisexual, and transgendered (LGBT) communities; and rural locations. Participants indicated that the lack of diverse representation was problematic and is an area for improvement. Additionally, a minority of participants formally serve or did serve on the Standards Advisory Committee, thus most participants did not have an opportunity for prolonged and meaningful engagement with the standards creation process. These factors contribute to an overall sense that the standards creation process was not as transparent as it could have been, providers are not as informed about the process as they could be, and the Standards Advisory Committee does not adequately represent all the groups that it should. In order to address these gaps and ultimately increase legitimacy, it appears that increasing the diversity of the Standards Advisory Committee and educating providers about the structure of the Standards Advisory Committee and its decision making process may be especially valuable in building trust and confidence.

The lack of discussion related to perceived valence of norms related to standards among providers poses several interesting areas for further inquiry. First, the current study conceptualized norms as descriptions of the standards as viewed positively or 
negatively in the field. It is possible that asking only whether participants believed other providers viewed the standards as positive or negative was not ideal and future research should assess other aspects of norms, such as what participants see others doing in the field. Despite the possible measurement inadequacy of assessing norms by asking about perceived valence of views towards the standards among providers, a post-hoc code was useful in gaining further knowledge about discussion in the field. The use of a post-hoc discussion code indicated that key program staff participate in conversations with other providers in the field, though the discussions tend to be educational and focused on information sharing rather than centered on whether the standards are particularly positive or negative. With the available conceptualization of norms, it is evident that comments related to norms were equally positive and negative. Thus, it appears that norms in the field are mixed. As interaction is required for the transmission of norms (Cialdini \& Trost, 1998) encouraging and providing opportunities for providers to interact with others in the community collaborative response (e.g., criminal justice system, victim advocates) may be useful in sharing norms from other perspectives as they relate to standards.

Finally, the findings related to policy logic were not surprising given the current lack of evidence-based practices in the field of batterer intervention. Participants tended to believe that the standards were based on available best practices but what is known related to best practices may be limited to majority cultures. Most participants did not believe the standards were developed from evidence-based practice. This has been a major critique of standards among the research community (Austin \& Dankwort, 1999; 
Holtzworth-Munroe, 2001) and it is clearly a critique shared by providers. The only way to alleviate this concern and build policy logic is to create a better empirical research base that can be used to develop standards. This is important for two reasons. First and foremost, building a better evidence-base for batterer intervention work should lead to better outcomes for the men and subsequently for their partners. Testing different intervention techniques and approaches to determine which aspects of program functioning should be dictated by standards and what those specific mandates should include would be valuable to ensure the components of the standards are tied to decreased recidivism and increased victim safety. This would not only ensure the components of standards will provide for better outcomes, but also would remove components that are not shown to be effective, potentially providing increased provider autonomy for aspects of program functioning that will not compromise victim safety. Second, increasing the evidence behind the components of standards may change how they are viewed amongst providers and improve perceptions related to policy logic. If components of the standards are based in empirical evidence, it may be more difficult for providers to disagree with their inclusion. Evidence suggesting that specific provisions in the standards (e.g., program length) are associated with lowered recidivism and increased victim safety would make their inclusion in the standards less controversial as there would be a clear link between the component and desired outcomes for program participants.

A review of the legitimacy literature (Tyler, 2006) suggests that legitimacy impacts how a policy or administrative body is viewed (i.e., procedural justice, social norms, and policy logic), which in turn impact adherence to a given policy such that 
increased legitimacy is associated with increased implementation (Tyler \& Huo, 2002; Stevenson et al., 2002; Wallner, 2008). Thus, it was predicted that legitimacy would differentiate high and low compliance programs. This prediction was not supported, as all participants reported relatively low levels of legitimacy regardless of compliance group. There are several plausible explanations for the lack of support for this hypothesis. First, there was a large difference observed between the two groups on one aspect of legitimacy. After separating the construct of legitimacy into its three components (i.e., procedural justice, norms, and policy logic), it was evident that low compliance programs made fewer comments indicative of perceiving procedural justice and policy logic. While this was the case, the large difference in the proportion of positive norms discussed in the two groups impacted the legitimacy ratio such that low compliance programs had higher legitimacy ratios on average. This indicates that it may be important to consider the aspects of legitimacy in isolation when attempting to understand how legitimacy functions across the two groups. Though it is plausible to suggest that increasing procedural justice and policy logic may be important for low compliance programs while increasing positive norms may be important for high compliance programs, it is important to recognize that all three of these components of legitimacy were relatively low across the groups. Thus, in order to increase legitimacy, which may in turn influence implementation, all three components of legitimacy should be targeted across all programs.

Second, it is possible that the way legitimacy was assessed in the current sample contributed to the low levels of legitimacy and thus revised measures may make the 
relationship between compliance and legitimacy more apparent. Specifically, norms regarding the state standards were operationalized to include perceptions of the standards as positive or negative in the field. This provided useful information about the subjective and injunctive norms surrounding standards but neglected to gather information about descriptive norms. Thus, while providers may have believed others had negative attitudes towards the standards, which was reflected in the relatively high prevalence of negative norms, findings from this study cannot speak to whether providers believed others were implementing the standards despite their negative attitudes. In retrospect, it may have been valuable to assess descriptive norms including the perceived practices of other providers in the field in order to more comprehensively assess norms and subsequently assess legitimacy.

Third, it is possible that those from high compliance programs, who were also those with more actual control over the standards, have negative views about legitimacy that are qualitatively different than low compliance programs. For instance, while high compliance program participants' perceptions of low legitimacy stemmed from direct exposure to the standards process, low compliance program participants' perceptions were typically based on a hypothetical understanding of the standards creation process. Thus, while perceptions based on real or hypothetical understanding of the standards creation process are relevant to legitimacy, they may be capturing different ends of the spectrum related to this construct. It is possible that perceptions of legitimacy based on actual exposure to the policy process and key individuals may provide a different 
perspective on legitimacy than hypothetical musings related a body and process with which they are unaware.

The relatively low degree to which participants endorsed legitimacy of the standards, standards creation process, and the Standards Advisory Committee, suggest it may be valuable to attempt to increase legitimacy across all programs. In order to increase legitimacy, several steps could be taken. First, providing program representatives with knowledge as to how the standards were developed and how the Standards Advisory Committee functions may provide more confidence in the procedural justice of the standards. If providers gained knowledge that the standards were built through collaboration with multiple stakeholders and were informed that they are able to provide feedback to the Standards Advisory Committee, they may feel that this process is fairer. The next two components of legitimacy may be more difficult to impact in a targeted way. Participants tended to have few comments related to norms. Providing opportunities to talk about the standards may allow providers to gain a better sense of how they are viewed in the field and create a consensus around the norms related to the standards. It is possible that providing a space for conversation may lead to the development of positive norms but it is also possible it could introduce negative norms. Thus, educational efforts designed to address transparency are needed to promote procedural justice and should be undertaken along with allowing and encouraging conversation regarding norms. This could be achieved through an increase in networking among BIP providers that was suggested by interview participants. Finally, the lack of policy logic may be a difficult hurdle to overcome. Current research is not definitive as to 
the effectiveness of BIPs or the mechanisms that promote effectiveness (Babcock et al., 2004; Feder \& Wilson, 2005; Feder et al., 2008; Gondolf, 2002). Additionally, research has not established which specific components of the standards are effective in reducing recidivism and improving victim safety. Because of this it is difficult to root the standards in evidence. In order to address this concern, more research should be done to examine what aspects of program functioning are related to positive outcomes. Given the difficulties conducting rigorous research in this area (Gondolf, 2002), it is imperative that multiple outcomes are considered (e.g., increased victim safety, increased victim knowledge, recidivism, etc.) in order to get a better sense of which approaches impact which outcomes. Without having this base of research, it will be difficult to increase policy logic.

Former providers. When experiences were quantified, current and former providers were similar in regards to some experiences (i.e., actual control and absoluteness) but differed in relation to other experiences (i.e., negative attitude change, negative attitude maintenance, and policy logic). In terms of commonalities, actual control and absoluteness were experienced to a similar degree in both groups. This is informative as, regardless of group, it appears that most participants reported more instances of not having actual control over the standards. Thus, it does not appear to be the case that former providers had less actual control than their current provider colleagues. This suggests that having control over and familiarity with the development and refinement of the standards may not be associated with whether or not a program ceases to provide services. Similarly, absoluteness appeared to be similar across the 
groups, indicating that those who stopped providing services did not perceive the standards as more or less absolute than the current providers.

While this is the case, former providers reported more positive initial and current responses to the standards. The relatively higher initial response observed among former provider participants indicates that when the standards were developed, they had a generally high opinion of them. This makes sense, as these participants did not believe the standards were the primary or only reason for program closure and most indicated they would like to provide BIP services in the future. The higher current response among former provider participants may be due to a truly more positive perception of the standards or may be due to the fact that these providers are no longer impacted by the standards and because of that they do not have to think about the implications of the standards on a day-to-day basis. The different pattern of responses related to policy logic may be the result of the condensed interview structure or the fact that these providers have not been recently immersed in the BIP community and may not be aware of the current state of research and practice. Current provider participants were more descriptive and explanatory when describing the policy logic of the standards while former provider participants tended to just state whether they believed they were or were not based in best practice or based in evidence without exploring the distinction between the two.

Finally, a slightly larger proportion of the former provider participants reported maintenance of negative attitudes towards standards (40\% vs. 31\%) and no participants reported experiences of changing negative attitudes towards standards. As maintenance of negative attitudes may suggest reactance occurred, the reactance literature is useful to 
consider. The reactance literature suggests that when individuals are faced with a freedom-limiting policy they are motivated to maintain those freedoms and may resist making changes in order to accomplish this (Brehm, 1966; Dillard \& Shen, 2005; ErcegHurn \& Steed, 2011; Mann, 2010). Perhaps when faced with the numerous changes that were required in areas that they may not agree, coupled with other factors, these former providers determined that the decision to cease services was most logical. As was observed among current providers, absoluteness was not consistent among these providers. This finding does not support the prediction that absoluteness differs those who change and maintain negative attitudes towards the standards, suggesting that maintaining negative perceptions towards the standards is not impacted by the extent to which former providers viewed the standards as required.

The exploration of former provider experiences highlights that while current and former providers may have some unique responses to standards (i.e., change in negative attitudes, maintenance of negative attitudes, and policy logic), there are also commonalties (i.e., actual control and absoluteness). Additionally, former providers do not attribute the stopping of BIP services to standards exclusively and currently view the standards as primarily positive. Further, former providers were not as vocal or informed about the current state of the standards since they had not been involved for some time. Given this, while former providers shed light on the impact of standards on program practices and characteristics, current providers were more vocal and descriptive about their experiences. 
Policy implementation. The identification and explanation of social psychological reactions to the introduction of a policy were valuable for identifying individual characteristics and perceptions that could be targeted for intervention. Experiences related to policy implementation were also evaluated in order to get a clear description of the impact of standards at the program level, the impact of different social psychological constructs on the process of policy implementation, as well as the barriers and facilitators experienced during implementation.

Top-down and bottom-up implementation analysis. The integration of both phases of the study provides insight into top-down and bottom-up implementation of standards. Top-down implementation occurs when those in positions of power generate policy decisions and are responsible for ensuring they are carried out, while bottom-up implementation occurs when those impacted by implementation are able to guide policy and implementation decisions (Barrett, 2004; Elmore, 1979; Matland, 1995). Those in the field of policy implementation have advocated for the integration of these two frameworks (O’Toole, 2004) in understanding and analyzing the implementation process. It is evident that the ways in which the standards in Oregon were mandated and created involved aspects of both frameworks. Specifically, the mandate for standards was introduced at the state level through legislation. While this was the case, the development and refinement of standards have provided the opportunity for providers to give input and help shape the content and scope of the standards. This provided an opportunity to assess how these two frameworks coincide and contribute to the understanding of policy implementation in this context. Specifically, the findings of this study demonstrate that 
utilizing an exclusively top-down or bottom-up approach to understanding BIP standards implementation is inadequate. The actual control over the standards appears to be a valuable indicator of whether their implementation should be conceptualized as top-down or bottom-up. The policy implementation process appears to be experienced as a topdown process for those who were not involved with or aware of the formation of the standards. These individuals were simply given the regulations to which they were expected to adhere and were not involved in participatory exchange regarding the content of the standards. Conversely, those who participated on the Standards Advisory Committee, provided input to members of the Standards Advisory Committee, or were able to keep abreast of the development process appear to have experiences more aligned with a bottom-up analysis of policy implementation. These findings are consistent with current literature surrounding policy implementation analysis, which points to the complementary nature of these approaches (O'Toole, 2000; 2004). Specifically, understanding experiences that align with the top-down implementation structure, such as legitimacy, as well as experiences that align with the bottom-up implementation structure, such as actual and perceived control, are both valuable in understanding implementation. This knowledge is particularly useful in determining the best approach for further study of policy implementation related to BIPs and perhaps other policies that are mandated legislatively but carried out with input from those impacted. Specifically, if future questions regarding implementation in this context arise, researchers and policymakers should draw on experiences consistent with both top-down and bottom-up implementation. Future efforts to understand policy implementation among BIPs should 
take the diversity of implementation processes into account when determining the most appropriate questions to ask to capture implementation.

Implementation strategies. Comments related to implementation strategies revealed that building relationships among other members of the community collaborative response (e.g., victim advocates) is crucial to implementation. Specifically, three categories of strategies that were identified in the interview data (i.e., attending trainings, increasing collaborations, and hosting trainings) center on improving, creating, or capitalizing on collaborative relationships. Given that the standards have a clear emphasis on the importance of collaboration (ODOJ, 2009) it is perhaps not surprising that participants commonly reported strategies for implementation related to collaboration. While many strategies centered on collaboration, the most prevalent strategy required participants to make changes to their program practices or policies. Similarly, $15 \%$ of participants reported hiring new staff to better meet the requirements of the standards. Given that program changes were an important implementation strategy, determining the quality and extent of changes was necessary.

Difficulty and ease changing policies and characteristics. The discussion of program features that are more and less difficult to change highlights the unique experience and context of each program. First, it is evident that most participants have attempted to make changes in their programs to more thoroughly implement the standards. Conversation about the different changes they have made or have attempted to make was descriptive and thorough, indicating that many participants do make efforts to change. Second, many of the same components were raised as easy and difficult 
components of the standards to implement (e.g., training requirements, collaborations, program length). This indicates that components of the standards that are relatively straightforward for one program may be highly problematic for implementation in another program. The diversity in components described as easier and harder to implement calls for an individualized effort to aid programs in improving implementation. Specifically, while one program may be in need of consultation surrounding collaboration, another program may lack capacities for mixed gender cofacilitation. Thus, if full implementation were a goal for the Standards Advisory Committee or leaders in Oregon, then it appears that the approaches used to increase implementation should take the diversity of program needs into account. Not only would this account for differences in needs across programs, but this would also prevent resources from being wasted when programs have already successfully implemented some components of the standards.

Compliance enablers and barriers. Taken together the common thread between the reported enablers to compliance appears to be related to the acquisition of knowledge and relationships. Across all types of enablers, there is a connection to having the necessary knowledge of the standards to make informed choices about program characteristics, as well as having access to positive collaborative relationships that are vital to pursuing implementation. It is difficult to tease apart whether having knowledge or relationships is most vital as it appears that for some participants having knowledge of the standards motivated the creation or strengthening of collaborative relationships, while for some participants collaborative relationships were vital in providing knowledge about 
the standards. These findings indicate that in order to encourage implementation, providers may benefit from increased knowledge about the explicit requirements and recommendations of the standards, as well as the opportunity to connect to other key members of the community collaborative response. Together, these activities would help facilitate the majority of enablers to implementation described by participants and in turn possibly increase implementation.

When the barriers to compliance are considered in combination, it is obvious that the types of barriers experienced by participants are diverse and likely relate to the unique context of each program. For instance, experiencing content barriers such as understanding the definition and role of an LSA was voiced by participants located in areas where the LSA has used their designation to make substantive changes to the regulations set forth by the standards. This barrier was not voiced for those in areas with an LSA that has decided to require compliance with the standards as they were written. Similarly, barriers such as rural location and small program size were voiced most commonly by those located in rural locations. Thus, it appears that in order to address barriers to compliance, distinct approaches may be most helpful for programs in different contexts. For instance, negotiation between the LSA and Standards Advisory Committee regarding the content of the standards may be useful for some programs, while building capacities to establish relationships for those that are geographically isolated may be useful for other programs.

When the enablers and barriers to compliance are considered together, it appears that enablers for some programs are barriers for other programs. This is most evident in 
the case of the agency barriers, where not only is collaboration with agencies more problematic for some programs, but collaboration with certain agencies may constitute an enabler while collaboration with other agencies may constitute a barrier within the same program. Indications that collaborative relationships enabled compliance focused on the utility of open, communicative, and supportive relationships among relevant agencies. These comments highlighted the importance of information sharing and constructive feedback when attempting to implement the standards. Conversely, agency barriers associated with implementation were focused on difficulties experienced when relationships are unbalanced in terms of power, contentious, or non-existent. Together, these comments indicate that collaborative relationships are immensely beneficial when they are supportive but challenging relationships can actually inhibit implementation. This suggests that work to bridge relationships and provide examples as to how positive collaborative relationships could function would be beneficial.

Support needed. Program representatives offered diverse suggestions to support achieving compliance that fell into four categories: changes to the standards and standards materials, collaborative relationships, monitoring and enforcement, and funding. First, changes to the standards and standards materials as a tool to enable greater compliance could be considered. Given that numerous participants felt uninformed about the standards process and found the standards difficult to understand, the gains in compliance that may be associated with the creation of a plain language synopsis of the standards distributed to programs statewide, may be worth the effort required to develop these materials. This product could include a simple description of the rationale behind 
the standards and the standards creation process, how and by whom they are monitored, a bulleted list of required program characteristics, and a bulleted list of suggested program characteristics. This product could be immensely beneficial for current providers, as well as providers that begin providing BIP services in the future.

Second, to enable greater compliance suggestions for community collaboration (e.g., creating a professional organization for providers, a statewide provider network, a BIP conference, developing listservs for providers) could be considered. The use of a conference to train batterer intervention providers may be particularly valuable as research demonstrates that providing training or technical assistance regarding the policy or program to be implemented is associated with better implementation (Dufrene et al., 2005; Durlak \& DuPre, 2008; Sterling-Turner et al., 2002). The goal of training in this context would be to prepare providers to make changes in their programs including practical suggestions to enable changes and efforts to increase self-efficacy and motivation to adhere to the standards (Dufrene et al., 2005; Durlak \& DuPre, 2008; Sterling-Turner et al., 2002). While training may be especially valuable, the resources required to enact the suggestions raised by participants vary, with some suggestions requiring relatively few state resources and others requiring a more substantial commitment. For example, setting up an e-mail listserv or distributing regular newsletters to providers would likely be relatively low in cost while developing and executing a statewide conference may require more resources. Thus, these suggestions provide the Standards Advisory Committee with options that can be utilized depending on the extent to which resources are available. 
Third, participants indicated that increased monitoring and enforcement would likely enable greater compliance. The suggestion to increase monitoring coincides with empirical evidence suggesting that implementation is more successful when the process is monitored as compared to when there is no monitoring processes in place (DuBois, Holloway, Valentine, \& Cooper, 2002; Durlak \& DuPre, 2008; Smith, Schneider, Smith \& Ananiadou, 2004). While monitoring and enforcement in one form or another may be valuable for increasing compliance with standards (DuBois et al., 2002; Durlak \& DuPre, 2008; French \& Raven, 1959; Smith et al., 2004), discussion in the BIP community has revealed that these efforts are difficult given the financial realities in the state. Specifically, participation in the BIP community and observations of discussion in provider meetings has revealed that ideas related to monitoring and enforcement have been assessed but have not yet been feasible. Participants indicated that there had been conversations and progress to develop a monitoring system within an organization that focused on monitoring of other types of businesses that are required to follow state or local standards. This process was halted when the monitoring agency indicated they were unable to take on the increased workload associated with monitoring BIPs and has not been reinitiated in any formal way. Comments from those well acquainted with this situation indicated that further steps towards monitoring have not been made due to financial constraints. While this is the case, several participants noted that participation in the BIP survey served as a form of monitoring and encouraged them to evaluate their practices as they relate to the standards. This indicates that while state-level monitoring 
may not be financially possible, administering the BIP Survey at regular intervals may serve as a valuable proxy for formal monitoring.

Finally, participants reported that increased funding would be valuable for achieving greater compliance. Greater funding would be useful because it would likely increase programs' ability to achieve components of the standards that rely on having resources. Funding could be used to hire additional facilitators in order to allow for mixed-gender co-facilitation, to ensure facilitators receive training, to compensate staff time for the development of required materials, and to pay staff to attend meetings with community partners. While this is the case, the lack of ideas regarding where additional funding may come from suggests that this approach may be less feasible. Thus, despite the potential benefits that may occur with increased funding, further thought as to how financial resources would be acquired and distributed is necessary.

Policy implementation and social psychology. When social psychology and policy implementation are considered together, numerous conceptual linkages and opportunities for greater exploration arise. The current study suggests that the responses and reactions of key staff, including experiences related to actual control, perceived control, and attitude change and maintenance, are useful for understanding the extent to which an entire program implements a policy. Due to the wide applicability of these phenomena, it is possible to examine their role in other studies of policy implementation, thus allowing a broader investigation of factors that impact implementation that are applicable in diverse contexts. Further, these social psychological constructs are not strictly theoretical and can be tied to many concrete recommendations to improve 
implementation. This makes them practically useful for generating recommendations, in addition to being theoretically relevant for building a base of knowledge about the role of key individuals in increasing program level implementation. Future research should expand upon this framework and attempt to examine how the fields of social psychology and policy implementation can build upon one another to develop a base of knowledge that is both theoretically relevant and valuable for real-world decisions.

Practice and policy. This project has the potential to make several important contributions to policy and practice. O'Toole $(1986 ; 2004)$ suggests that policy implementation research has the ability to be practically useful in identifying problematic elements of the translation of policy into action and making others aware of the problems. This study contributes to the field of batterer intervention by addressing the potential of policy implementation research to describe the process by which standards were implemented and the reactions, responses, barriers and facilitators therein. First, this study aimed to integrate the ideals of social action research. Steps were taken to contribute in practical ways to the community throughout the research process. The BIP directory is a concrete example of a product that was developed as a result of the project. This directory is a practical and useful resource in the community, as it is the only source of statewide referral information. The directory is currently used by many organizations in Oregon including courts, probation, BIPs, and advocacy agencies. These agencies use the directory to refer clients and network programs across the state. It is currently posted on a webpage for the Oregon Department of Justice (ODOJ, 2010) and the Oregon Department of Human Services (DHS, 2013). The use of the directory by ODOJ and 
DHS, as well as use among BIP providers, points to the need for accurate and current information. The preliminary information gained through initial phone calls was utilized to update the Oregon BIP directory. Thus, this project had the practical implication of providing an up-to-date resource for any interested parties in Oregon or elsewhere, thus giving back to the community and upholding the ideals of social action research (Lewin, 1946). Additionally, the findings from the study will be presented back to policymakers and this may potentially inform the policy development process. The act of giving information back to the community corresponds to the description of social action research outlined by Lewin (1946) due to the focus on providing information that is not only useful theoretically, but in practice as well. Further, this process has the potential to begin and encourage dialogue among program staff and policymakers, as it is a first step towards that process.

Second, knowledge gained regarding the extent to which programs have implemented the standards can be utilized by policymakers to inform decisions about developing a monitoring or enforcement system. Currently, Oregon does not utilize a monitoring or enforcement system. Research demonstrates that monitoring is associated with better implementation (DuBois et al., 2002; Durlak \& DuPre, 2008; Smith et al., 2004). Further, some participants indicated that having a supportive and non-punitive monitoring mechanism would be helpful in increasing compliance. While creating a monitoring system may be a large undertaking, if complete compliance is important, it may be useful. Further, due to the desire for a supportive and non-punitive system, it is possible that monitoring could occur somewhat informally such as through surveys or 
telephone consultation, rather than requiring a full certification process (e.g., site visits to verify self-reports with consequences for discrepancies). This study may serve as a platform to begin new discussion about whether and what type of monitoring or enforcement system may be valuable.

Third, the content of interview responses provided by BIP representatives revealed components of the standards with which participants agree and were able to implement, as well as areas of disagreement or challenge with implementation. The Oregon state standards are periodically refined and revised to account for developments in the field of batterer intervention and feedback from providers or others involved in the community collaborative response. As standards evolve, policymakers should pay attention to the experiences and perceptions of providers to help guide future iterations of the standards. For instance, current efforts have been undertaken to modify the length requirement of the standards and it is possible that further changes will be proposed in the future. The current study could serve as an important source of information to inform these efforts. For instance, this information could be used to modify standards to become more aligned with the experiences of providers. While many components of the standards may be inappropriate or dangerous to modify given their theoretical or empirical linkage to victim safety, there are some areas where changes could be considered. One example provided by participants was related to the lack of standards for perpetrators in same sex relationships or for female perpetrators. Participants believe that these are different populations who require different intervention techniques than those typically used with males who are violent towards a female victim. These providers reported that a set of 
standards to help guide practice when they do get female or LGBT clients would be helpful. Thus, while not every component should be altered to align with program practices or provider opinions, there are likely some areas where provider experiences could inform changes to the standards.

While altering the content of the standards is one approach that may be useful for a small portion of the components, a major theme of these findings points to the need for education and networking among providers. Promoting greater access to those involved in the IPV community may not only address some of the barriers and supports needed that were identified by participants, but it also may bolster actual and perceived control as these providers become better acquainted with others in the field. Thus, these steps may be necessary to encourage implementation with components that currently do not align with participants' beliefs but are rooted in victim safety or thought to be the most appropriate practice currently available.

Fourth, the current study included program directors and key program representatives as participants, ensuring those who are most responsible for enacting steps relevant to implementation were involved in the study. The psychology of these individuals is immensely important as their choices and decisions ultimately determine the ways in which the standards are implemented and extent to which compliance is achieved. The study in and of itself could be viewed as an intervention given that participants may have gained greater knowledge about the standards and felt connected to someone in the BIP community (i.e., the researcher). The implementation literature suggests that the attitudes and perceptions of those responsible for implementation 
influence the extent to which implementation occurs (O'Toole, 1986; 2000; 2004). It is plausible that participation in the study may have encouraged participants to think more critically about the standards and the extent to which their program is or is not in compliance, which may in turn influence subsequent implementation strategies and overall compliance. For example, participation in the 2011 BIP Survey educated one participant about the need for written guidelines and procedures. This new knowledge prompted the participant to develop written protocols for their program. If the individual who completed the survey did not have the power to enact program change, the new knowledge may not have been translated into action. Thus, the use of key representatives was crucial to ensure that those who are asked to think deeply about their experience with implementation, as well as their critiques and endorsements of the standards, are also those who are best poised to use the experience to move forward and make changes in their program or the community.

Fifth, this project may have been beneficial to participants because it provided them an opportunity to make their thoughts, opinions, and experiences known to those making policy decisions. Program representatives had the opportunity to share what they have experienced and which components of the standards align with their beliefs and practices. As Rappaport (1998) argued, providing an opportunity for participants to discuss their experiences can be an empowering experience. Participants had the opportunity to describe their stories and experiences related to the implementation of standards to policymakers, thus amplifying their voices (Rappaport, 1998). The act of storytelling in and of itself, as occurred during the interview process, can be a catalyst for 
social change (Rappaport, 1998). One concrete example of this project being a catalyst for change includes the fact that two providers contacted the researcher after the interviews were complete to share additional information or inform the researcher that they had made steps towards greater implementation. This project gave those directly affected by the policy an avenue to discuss their views and present their insights to policymakers. Individuals who develop the standards will have the opportunity to hear the voices of those impacted by the standards and better understand how they are perceived in the field. Out of this process emerges the potential to significantly impact the dialogue between policymakers and BIP directors.

While the current study serves as a platform to amplify participant voices, the extent to which the Standards Advisory Committee will value their opinions and beliefs remains unknown. The Standards Advisory Committee demonstrated interested in this study and provided suggestions to make this study relevant to their needs but it is possible that the voices of providers will not necessarily be valued or taken seriously. The goals and composition of the Standards Advisory Committee will likely play an important role in determining whether and how the information gained in the current study will be utilized. If the Standards Advisory Committee is interested in working with providers to ensure the standards meet the needs of clients and are feasible for providers, this study provides numerous avenues to pursue that goal. If that is not a goal of the committee or if the committee members do not feel that BIP providers are best poised to provide this information, their use of this study may be limited. It will be interesting to 
observe how the amplified voices of BIP providers are or are not taken seriously by the committee and integrated into future decisions.

Additionally, it is possible that the experience of making their thoughts known to policymakers might have provided the participants with an increased sense of control over the content of the standards and encourage them to become more involved in the standards refinement process in the future. This in turn may impact compliance such that programs change their practices to align with the standards, which theoretically could increase victim safety. Given the length of the interviews and the engagement that nearly every participant displayed, it was clear that participants had a great deal to say about their implementation experiences. It became apparent during the course of the interviews that providers would like others to recognize that the work they are doing is difficult and they are trying their best. For instance, one provider indicated what they wanted from the IPV community: "Just some acknowledgment or an appreciation for those who are doing batterers intervention work. For being willing to do the hard work". This quote highlighted the experience observed with many of the participants; just having a nonjudgmental individual interested in hearing about their work was valuable. Beyond being there to listen and better understand what providers do, this study will amplify their voices in a way that protects their identity and privacy, while still informing policymakers about their views and opinions related to the standards.

\section{Conclusions}

The study of policy implementation and the social psychological factors that may impact implementation in the context of BIPs is both innovative and timely. Specifically, 
there has yet to be a study investigating the implementation of state standards among BIPs and research and federal agency attention (e.g., National Institutes of Health (NIH)) have underscored the importance of implementation for the understanding of policy and program outcomes (Durlak \& DuPre, 2008; Eccles, Roy, Sales, Wensing \& Mittman, 2012; Meyers et al., 2012; NIH, 2013). Thus, this study serves as a crucial step towards understanding the process of implementation and current compliance that can be utilized for future studies aimed at determining the impact of standards on outcomes such as recidivism and victim safety.

The current study provides valuable information that can be used to further the development of theory, improve program practices, and inform policy. This study updates previous work (Boal, 2010) to determine the current practices and characteristics of BIPs in Oregon. This allows for greater insight into the extent to which programs are in compliance with state policy, as well as the areas for which compliance is greatest and most lacking. Next, this study sheds light onto the processes underlying program representatives' responses to state standards. Specifically, this study applies information from the actual control, perceived control, attitude change and maintenance (including the possible explanations of rationalization and reactance), absoluteness, and legitimacy literature to develop an understanding of these constructs among those implementing policy. Additionally, this study assesses how these constructs differentiate those who are most and least successful in implementing standards. Finally, this study provides practical insight regarding the process of policy implementation, including the enablers, barriers, and support needed to encourage greater compliance When considering the 
underlying social psychological constructs and the process of policy implementation together, this study suggests that participation in the policy process may be immensely valuable for developing actual control, perceived control and connections among community partners. Not only do these experiences appear to be useful in impacting program compliance, but they also call to the needs voiced by providers. 
Table 1.

Cohen's Kappa for Qualitative Coding

\begin{tabular}{|l|c|}
\hline \multicolumn{1}{|c|}{ Code } & Cohen's Kappa \\
\hline High Actual Control & 0.65 \\
\hline Low Actual Control & 0.87 \\
\hline High Perceived Ability & 0.63 \\
\hline Low Perceived Ability & 0.75 \\
\hline Positive Initial Response & 0.64 \\
\hline Negative Initial Response & 0.86 \\
\hline Positive Current Response & 0.76 \\
\hline Negative Current Response & 0.63 \\
\hline Absoluteness & 0.62 \\
\hline Non-absoluteness & 0.66 \\
\hline High Procedural Justice & 0.66 \\
\hline Low Procedural Justice & 0.55 \\
\hline Positive Norms & 0.88 \\
\hline Negative Norms & 0.65 \\
\hline High Policy Logic & 0.77 \\
\hline Low Policy Logic & 0.82 \\
\hline Implementation Strategies & 0.62 \\
\hline Implementation Ease & 0.66 \\
\hline Implementation Difficulty & 0.65 \\
\hline Facilitators & 0.74 \\
\hline Barriers & 0.62 \\
\hline Needed Support & 0.73 \\
\hline Social Action Research & 0.85 \\
\hline Limited Contact & 0.71 \\
\hline Discussion & 0.58 \\
\hline High impact & 0.62 \\
\hline Low impact & 1.00 \\
\hline Interest & 0.62 \\
\hline
\end{tabular}


Table 2.

Summary of Findings

\begin{tabular}{|c|c|c|c|}
\hline \multicolumn{3}{|c|}{ Research Question } & Finding \\
\hline$R Q 1$ & \multicolumn{2}{|c|}{$\begin{array}{l}\text { What are the current practices and policies of } \\
\text { BIPs in Oregon? }\end{array}$} & $\begin{array}{l}\text { On average, programs are in compliance } \\
\text { with } 75 \% \text { of the assessed components of } \\
\text { standards. }\end{array}$ \\
\hline \multirow[t]{10}{*}{$R Q 2$} & \multicolumn{2}{|c|}{$\begin{array}{l}\text { How do program representatives in Oregon react } \\
\text { and respond to state standards? }\end{array}$} & \\
\hline & $R Q 2 a$ & $\begin{array}{l}\text { To what extent do program } \\
\text { representatives report having actual } \\
\text { control over the content and } \\
\text { development of the standards? }\end{array}$ & $\begin{array}{l}\text { On average, } 54 \% \text { of comments related to } \\
\text { actual control indicated participants did } \\
\text { not have actual control over the standards. }\end{array}$ \\
\hline & $R Q 2 b$ & $\begin{array}{l}\text { Do program representatives perceive } \\
\text { having control over the content and } \\
\text { development of the standards? }\end{array}$ & $\begin{array}{l}\text { On average, } 64 \% \text { of comments related to } \\
\text { perceived control indicated participants } \\
\text { did not have perceived control over the } \\
\text { standards. }\end{array}$ \\
\hline & $H 2 a$ & $\begin{array}{l}\text { Program representatives who primarily } \\
\text { report having actual control over the } \\
\text { standards will describe higher perceived } \\
\text { control as compared to those who } \\
\text { primarily report not having actual } \\
\text { control over the standards. }\end{array}$ & $\begin{array}{l}\text { Consistent with expectations, those who } \\
\text { primarily reported having actual control } \\
\text { over the standards had perceived control } \\
\text { ratios } 23 \% \text { higher than those who } \\
\text { primarily reported not having actual } \\
\text { control over the standards. Though, this } \\
\text { difference was not statistically reliable. }\end{array}$ \\
\hline & $R Q 2 c$ & $\begin{array}{l}\text { Do program representatives describe } \\
\text { responses to the standards consistent } \\
\text { with the phenomenon of } \\
\text { rationalization? }\end{array}$ & $\begin{array}{l}\text { Four participants }(31 \%) \text { reported shifting } \\
\text { their initial negative attitudes towards the } \\
\text { standards to be primarily positive. }\end{array}$ \\
\hline & $R Q 2 d$ & $\begin{array}{l}\text { Do program representatives describe } \\
\text { responses to the standards consistent } \\
\text { with the phenomenon of reactance? }\end{array}$ & $\begin{array}{l}\text { Four participants }(31 \%) \text { reported } \\
\text { maintaining their initial negative attitudes } \\
\text { towards the standards over time. }\end{array}$ \\
\hline & $R Q 2 e$ & $\begin{array}{l}\text { Do program representatives view the } \\
\text { standards as an absolute policy? }\end{array}$ & $\begin{array}{l}\text { On average, } 58 \% \text { of comments related to } \\
\text { absoluteness indicated participants } \\
\text { viewed the standards as absolute. }\end{array}$ \\
\hline & $H 2 b$ & $\begin{array}{l}\text { Program representatives who respond to } \\
\text { the standards with rationalization will } \\
\text { view the standards as more absolute } \\
\text { than program representatives who } \\
\text { respond to the standards with reactance. }\end{array}$ & $\begin{array}{l}\text { Contrary to expectations, absoluteness did } \\
\text { not differentiate those who changed their } \\
\text { initial negative attitudes towards the } \\
\text { standards (i.e., a proxy for rationalization) } \\
\text { and those who maintained their initial } \\
\text { negative attitudes (i.e., a proxy for } \\
\text { reactance) towards the standards. }\end{array}$ \\
\hline & $R Q 2 f$ & $\begin{array}{l}\text { Do program representatives perceive } \\
\text { the standards and the process by which } \\
\text { the standards were created as } \\
\text { legitimate? }\end{array}$ & $\begin{array}{l}\text { On average, } 60 \% \text { of comments related to } \\
\text { legitimacy indicated participants did not } \\
\text { view the standards and their creation as } \\
\text { legitimate. }\end{array}$ \\
\hline & $R Q 2 g$ & $\begin{array}{l}\text { How have state standards impacted BIP } \\
\text { closures across the state of Oregon? }\end{array}$ & $\begin{array}{l}\text { On average, } 73 \% \text { of comments related to } \\
\text { the impact of standards on program } \\
\text { closure indicated participants believed the } \\
\text { standards were not responsible for } \\
\text { ceasing BIP services. }\end{array}$ \\
\hline
\end{tabular}




\begin{tabular}{|c|c|c|c|}
\hline \multicolumn{3}{|c|}{ Research Question } & Finding \\
\hline$R Q 3$ & \multicolumn{2}{|c|}{$\begin{array}{l}\text { How have programs in Oregon implemented } \\
\text { state standards? }\end{array}$} & \\
\hline & $R Q 3 a$ & $\begin{array}{l}\text { What specific strategies have program } \\
\text { representatives used to implement the } \\
\text { standards? }\end{array}$ & $\begin{array}{l}\text { Participants named a total of } 57 \\
\text { implementation strategies }(M=4.38, S D \\
=3.04) \text {, which included reading the } \\
\text { standards, changing practices and } \\
\text { policies, attending trainings, hosting } \\
\text { trainings, building relationships, and } \\
\text { hiring new staff. }\end{array}$ \\
\hline & $R Q 3 b$ & $\begin{array}{l}\text { Which program policies and } \\
\text { characteristics are described as } \\
\text { relatively easy and relatively difficult to } \\
\text { implement by program representatives? }\end{array}$ & $\begin{array}{l}\text { Participants named a total of } 67 \text { program } \\
\text { characteristics that were easy to change } \\
(M=5.15, S D=3.58) \text {, and } 69 \\
\text { characteristics that were difficult to } \\
\text { change }(M=5.31, S D=3.15) \text {. Most } \\
\text { commonly, participants indicated } \\
\text { program length and alignment with the } \\
\text { philosophical orientation of the standards } \\
\text { were easy to implement while the most } \\
\text { common components that were difficult } \\
\text { to implement were related to access and } \\
\text { resources (e.g., collaboration, training, } \\
\text { staffing). }\end{array}$ \\
\hline & $R Q 3 c$ & $\begin{array}{l}\text { What factors enable BIPs' compliance } \\
\text { with state standards? }\end{array}$ & $\begin{array}{l}\text { Participants named a total of } 53 \text { enablers } \\
\text { to compliance }(M=4.08, S D=3.52) \text {, } \\
\text { which primarily included strong } \\
\text { collaborative relationships, useful } \\
\text { activities, and program or staff } \\
\text { characteristics. Participants also indicated } \\
\text { participation in the study was valuable to } \\
\text { encourage compliance. }\end{array}$ \\
\hline & $R Q 3 d$ & $\begin{array}{l}\text { What factors are barriers to BIPs' } \\
\text { compliance with state standards? }\end{array}$ & $\begin{array}{l}\text { Participants named a total of } 105 \text { barriers } \\
\text { to compliance }(M=8.08, S D=5.12), \\
\text { which primarily included problematic } \\
\text { collaborations, achieving trainings, } \\
\text { difficulties interpreting the standards, and } \\
\text { challenges due to small size or rural } \\
\text { location. }\end{array}$ \\
\hline & $R Q 3 e$ & $\begin{array}{l}\text { What needs do program representatives } \\
\text { identify in order to successfully } \\
\text { implement the standards? }\end{array}$ & $\begin{array}{l}\text { Participants named a total of } 68 \\
\text { suggestions to improve implementation } \\
(M=5.23, S D=3.42) \text {, which included } \\
\text { improving collaboration among BIP } \\
\text { providers, creation of a monitoring } \\
\text { system, modification of the standards to } \\
\text { increase flexibility and comprehension, } \\
\text { and increased resources. }\end{array}$ \\
\hline
\end{tabular}




\begin{tabular}{|c|c|c|c|}
\hline \multicolumn{3}{|c|}{ Research Question } & \multirow[t]{2}{*}{ Finding } \\
\hline$R Q$ & \multicolumn{2}{|c|}{$\begin{array}{l}\text { Do the responses and reactions to standards } \\
\text { differ for programs with different levels of } \\
\text { compliance? }\end{array}$} & \\
\hline & $H 4 a$ & $\begin{array}{l}\text { High compliance program } \\
\text { representatives will describe } \\
\text { relatively more experiences of } \\
\text { actual control as compared to } \\
\text { low compliance program } \\
\text { representatives. }\end{array}$ & $\begin{array}{l}\text { Comments indicative of having actual } \\
\text { control over the standards was } 57 \% \\
\text { higher among high compliance program } \\
\text { participants compared to low compliance } \\
\text { program participants. This difference was } \\
\text { not statistically reliable. }\end{array}$ \\
\hline & $H 4 b$ & $\begin{array}{l}\text { High compliance program } \\
\text { representatives will describe } \\
\text { relatively more experiences of } \\
\text { perceived control as compared } \\
\text { to low compliance program } \\
\text { representatives. }\end{array}$ & $\begin{array}{l}\text { Comments indicative of having perceived } \\
\text { control over the standards was } 89 \% \\
\text { higher among high compliance program } \\
\text { participants compared to low compliance } \\
\text { program participants. This difference was } \\
\text { not statistically reliable. }\end{array}$ \\
\hline & $H 4 c$ & $\begin{array}{l}\text { High compliance program } \\
\text { representatives will describe } \\
\text { relatively more reactions } \\
\text { consistent with rationalization } \\
\text { as compared to low compliance } \\
\text { program representatives. }\end{array}$ & $\begin{array}{l}\text { Consistent with expectations } 75 \% \text { of the } \\
\text { participants who changed their initial } \\
\text { negative attitudes towards the standards } \\
\text { (i.e., a proxy for rationalization) were } \\
\text { representatives from high compliance } \\
\text { programs. }\end{array}$ \\
\hline & $H 4 d$ & $\begin{array}{l}\text { Low compliance program } \\
\text { representatives will describe } \\
\text { relatively more reactions } \\
\text { consistent with reactance as } \\
\text { compared to high compliance } \\
\text { program representatives. }\end{array}$ & $\begin{array}{l}\text { Consistent with expectations, } 100 \% \text { of the } \\
\text { participants who maintained their } \\
\text { negative attitudes towards the standards } \\
\text { (i.e., a proxy for reactance) were } \\
\text { representatives from low compliance } \\
\text { programs. }\end{array}$ \\
\hline & $H 4 e$ & $\begin{array}{l}\text { High compliance program } \\
\text { representatives will describe } \\
\text { relatively more perceptions of } \\
\text { the standards and process of } \\
\text { standards creation consistent } \\
\text { with legitimacy as compared to } \\
\text { low compliance program } \\
\text { representatives. }\end{array}$ & $\begin{array}{l}\text { Contrary to expectations, comments } \\
\text { indicative of legitimacy were } 18 \% \text { higher } \\
\text { among low compliance program } \\
\text { participants compared to high compliance } \\
\text { program participants. This difference was } \\
\text { not statistically reliable. }\end{array}$ \\
\hline
\end{tabular}


Table 3.

Compliance with Standards Ranked by \% in Compliance

\begin{tabular}{|c|c|c|c|c|c|}
\hline Standards Requirement & Sub-Requirements & $n$ & $M$ & $S D$ & $\begin{array}{c}\% \text { in } \\
\text { Compliance }\end{array}$ \\
\hline Group size & & 32 & 9.96 & 7.21 & $94 \%$ \\
\hline $\begin{array}{l}\text { Required program } \\
\text { length }\end{array}$ & & 34 & 44.13 & 11.95 & $94 \%$ \\
\hline \multirow[t]{3}{*}{$\begin{array}{l}\text { Liaison to criminal } \\
\text { justice system }\end{array}$} & & 34 & & & $94 \%$ \\
\hline & Communicate attendance & 33 & & & $94 \%$ \\
\hline & $\begin{array}{l}\text { Communicate program } \\
\text { outcomes }\end{array}$ & 33 & & & $70 \%$ \\
\hline \multirow[t]{3}{*}{$\begin{array}{l}\text { Contact with victim } \\
\text { advocacy agency }\end{array}$} & & 35 & & & $91 \%$ \\
\hline & Designated liaison & 32 & & & $91 \%$ \\
\hline & $\begin{array}{l}\text { Submit policies and } \\
\text { procedures }\end{array}$ & 32 & & & $69 \%$ \\
\hline \multirow[t]{5}{*}{$\begin{array}{l}\text { Completion } \\
\text { requirements }\end{array}$} & & 34 & 3.88 & .33 & $88 \%$ \\
\hline & Attendance & 34 & & & $100 \%$ \\
\hline & $\begin{array}{l}\text { Compliance with program } \\
\text { rules }\end{array}$ & 34 & & & $100 \%$ \\
\hline & Compliance with group rules & 34 & & & $94 \%$ \\
\hline & Accountability plan & 34 & & & $94 \%$ \\
\hline $\begin{array}{l}\text { Staff member attends } \\
\text { DV council meetings }\end{array}$ & & 30 & & & $87 \%$ \\
\hline $\begin{array}{l}\text { Collaboration with } \\
\text { BIPs }\end{array}$ & & 35 & & & $83 \%$ \\
\hline \multirow[t]{9}{*}{$\begin{array}{l}\text { Refrain from } \\
\text { prohibited program } \\
\text { curriculum }\end{array}$} & & 35 & .23 & .55 & $83 \%$ \\
\hline & View battering as addiction & & & & $100 \%$ \\
\hline & $\begin{array}{l}\text { Encourage ventilation } \\
\text { techniques }\end{array}$ & & & & $100 \%$ \\
\hline & $\begin{array}{l}\text { Blame battering on victim } \\
\text { qualities }\end{array}$ & & & & $100 \%$ \\
\hline & $\begin{array}{l}\text { View battering as bi- } \\
\text { directional }\end{array}$ & & & & $100 \%$ \\
\hline & $\begin{array}{l}\text { Require victim or partner } \\
\text { disclosure }\end{array}$ & & & & $97 \%$ \\
\hline & $\begin{array}{l}\text { Use actions of moral } \\
\text { superiority }\end{array}$ & & & & $97 \%$ \\
\hline & $\begin{array}{l}\text { Encourage victim or partner } \\
\text { disclosure }\end{array}$ & & & & $91 \%$ \\
\hline & $\begin{array}{l}\text { Support or recommend } \\
\text { couples/family counseling or } \\
\text { medication }\end{array}$ & & & & $91 \%$ \\
\hline
\end{tabular}




\begin{tabular}{|c|c|c|c|c|c|}
\hline Standards Requirement & Sub-Requirements & $n$ & $M$ & $S D$ & $\begin{array}{c}\% \text { in } \\
\text { Compliance }\end{array}$ \\
\hline \multirow{8}{*}{$\begin{array}{l}\text { Refrain from } \\
\text { endorsing prohibited } \\
\text { primary cause of } \\
\text { battering }\end{array}$} & & 35 & 3.71 & 2.36 & $80 \%$ \\
\hline & Substance use or abuse & & & & $94 \%$ \\
\hline & Anger & & & & $91 \%$ \\
\hline & $\begin{array}{l}\text { Client or victim mental health } \\
\text { problems }\end{array}$ & & & & $91 \%$ \\
\hline & Poor impulse control & & & & $89 \%$ \\
\hline & Unconscious motivation & & & & $89 \%$ \\
\hline & Past experience & & & & $86 \%$ \\
\hline & Low self-esteem & & & & $86 \%$ \\
\hline \multirow[t]{9}{*}{$\begin{array}{l}\text { Approved } \\
\text { intervention strategies }\end{array}$} & & 35 & 7.77 & .49 & $80 \%$ \\
\hline & Respectful confrontation & & & & $100 \%$ \\
\hline & Address tactics to justify & & & & $100 \%$ \\
\hline & $\begin{array}{l}\text { Stress impact of battering on } \\
\text { victims }\end{array}$ & & & & $100 \%$ \\
\hline & $\begin{array}{l}\text { Promote accountability for } \\
\text { controlling and abusive } \\
\text { behavior }\end{array}$ & & & & $100 \%$ \\
\hline & $\begin{array}{l}\text { Increase understanding of } \\
\text { causes, types and effects of } \\
\text { battering }\end{array}$ & & & & $97 \%$ \\
\hline & $\begin{array}{l}\text { Reinforce personal } \\
\text { responsibility }\end{array}$ & & & & $97 \%$ \\
\hline & $\begin{array}{l}\text { Reinforce appropriate beliefs } \\
\text { and behavioral alternatives }\end{array}$ & & & & $97 \%$ \\
\hline & $\begin{array}{l}\text { Increase recognition of } \\
\text { criminal aspect of behavior } \\
\text { and thoughts }\end{array}$ & & & & $86 \%$ \\
\hline $\begin{array}{l}\text { Victim information } \\
\text { only available to } \\
\text { designated BIP staff }\end{array}$ & & 34 & & & $79 \%$ \\
\hline $\begin{array}{l}\text { Distribute } \\
\text { informational } \\
\text { materials to victims }\end{array}$ & & 34 & & & $74 \%$ \\
\hline Contact with LSA & & 35 & & & $71 \%$ \\
\hline \multirow{3}{*}{$\begin{array}{l}\text { Refrain from } \\
\text { prohibited victim } \\
\text { contact }\end{array}$} & & 29 & & & $69 \%$ \\
\hline & $\begin{array}{l}\text { Informing victim about things } \\
\text { client said in group }\end{array}$ & 29 & & & $97 \%$ \\
\hline & Solicit information & 29 & & & $69 \%$ \\
\hline $\begin{array}{l}\text { Mixed gender co- } \\
\text { facilitation }\end{array}$ & & 31 & & & $58 \%$ \\
\hline
\end{tabular}




\begin{tabular}{|c|c|c|c|c|c|}
\hline Standards Requirement & Sub-Requirements & $n$ & $M$ & $S D$ & $\begin{array}{c}\% \text { in } \\
\text { Compliance }\end{array}$ \\
\hline \multirow[t]{2}{*}{$\begin{array}{l}\text { Victim advocacy } \\
\text { training }\end{array}$} & & 32 & & & $56 \%$ \\
\hline & $\begin{array}{l}\text { Facilitators meeting } \\
\text { requirement within program }\end{array}$ & 32 & .74 & .35 & \\
\hline \multirow[t]{2}{*}{ BIP training } & & 32 & & & $56 \%$ \\
\hline & $\begin{array}{l}\text { Facilitators meeting } \\
\text { requirement within program }\end{array}$ & 32 & .80 & .27 & \\
\hline $\begin{array}{l}\text { Collaboration in the } \\
\text { community }\end{array}$ & & 35 & & & $43 \%$ \\
\hline \multirow[t]{7}{*}{$\begin{array}{l}\text { Materials include all } \\
\text { required information }\end{array}$} & & 25 & 4.52 & 1.61 & $40 \%$ \\
\hline & $\begin{array}{l}\text { Includes victim advocacy } \\
\text { resources }\end{array}$ & 25 & & & $96 \%$ \\
\hline & $\begin{array}{l}\text { Includes BIP contact } \\
\text { information }\end{array}$ & 25 & & & $76 \%$ \\
\hline & Includes BIP description & 25 & & & $74 \%$ \\
\hline & $\begin{array}{l}\text { Includes statement of BIP } \\
\text { limitations }\end{array}$ & 25 & & & $72 \%$ \\
\hline & $\begin{array}{l}\text { Includes victims' rights } \\
\text { information }\end{array}$ & 25 & & & $68 \%$ \\
\hline & $\begin{array}{l}\text { Includes safety planning } \\
\text { information }\end{array}$ & 25 & & & $64 \%$ \\
\hline \multirow[t]{7}{*}{$\begin{array}{l}\text { Written policies and } \\
\text { procedures }\end{array}$} & & 35 & 4.31 & 1.49 & $26 \%$ \\
\hline & Program completion & 35 & & & $97 \%$ \\
\hline & Victim confidentiality & 34 & & & $88 \%$ \\
\hline & Victim safety & 35 & & & $77 \%$ \\
\hline & Victim contact & 34 & & & $71 \%$ \\
\hline & Storing victim information & 33 & & & $63 \%$ \\
\hline & Client transfers & 35 & & & $43 \%$ \\
\hline
\end{tabular}


Table 4.

Compliance with Program Logistics

\begin{tabular}{|c|c|c|c|c|c|}
\hline Standards Requirement & Sub-Requirements & $n$ & $M$ & $S D$ & $\begin{array}{c}\% \text { in } \\
\text { Compliance }\end{array}$ \\
\hline Group size & & 32 & 9.96 & 7.21 & $94 \%$ \\
\hline \multirow[t]{7}{*}{$\begin{array}{l}\text { Written policies and } \\
\text { procedures }\end{array}$} & & 35 & 4.31 & 1.49 & $26 \%$ \\
\hline & Victim safety & 35 & & & $77 \%$ \\
\hline & Program completion & 35 & & & $97 \%$ \\
\hline & Client transfers & 35 & & & $43 \%$ \\
\hline & Victim contact & 34 & & & $71 \%$ \\
\hline & Storing victim information & 33 & & & $63 \%$ \\
\hline & Victim confidentiality & 34 & & & $88 \%$ \\
\hline $\begin{array}{l}\text { Documentation of } \\
\text { program completion }\end{array}$ & & 35 & & & $66 \%$ \\
\hline $\begin{array}{l}\text { Required program } \\
\text { length }\end{array}$ & & 34 & 44.13 & 11.95 & $94 \%$ \\
\hline $\begin{array}{l}\text { Mixed gender co- } \\
\text { facilitation }\end{array}$ & & 31 & & & $58 \%$ \\
\hline \multirow[t]{5}{*}{$\begin{array}{l}\text { Completion } \\
\text { requirements }\end{array}$} & & 34 & 3.88 & .33 & $88 \%$ \\
\hline & Attendance & 34 & & & $100 \%$ \\
\hline & Compliance with program rules & 34 & & & $100 \%$ \\
\hline & Compliance with group rules & 34 & & & $94 \%$ \\
\hline & Accountability plan & 34 & & & $94 \%$ \\
\hline
\end{tabular}


Table 5.

Compliance with Training of Facilitators

\begin{tabular}{|l|l|c|c|c|c|}
\hline \multicolumn{1}{|c|}{ Standards Requirement } & \multicolumn{1}{|c|}{ Sub-Requirements } & $n$ & $M$ & $S D$ & $\begin{array}{c}\% \text { in } \\
\text { Compliance }\end{array}$ \\
\hline Victim advocacy training & & 32 & & & $56 \%$ \\
\hline & $\begin{array}{l}\text { Facilitators meeting } \\
\text { requirement within } \\
\text { program }\end{array}$ & 32 & .74 & .35 & \\
\hline BIP training & & 32 & .80 & .27 & $56 \%$ \\
\hline & $\begin{array}{l}\text { Facilitators meeting } \\
\text { requirement within } \\
\text { program }\end{array}$ & 32 & & \\
\hline
\end{tabular}


Table 6.

Compliance with Program Intervention Strategies $(n=35)$

\begin{tabular}{|c|c|c|c|c|}
\hline $\begin{array}{l}\text { Violation of Standards } \\
\text { Requirement }\end{array}$ & Sub-Requirements & $M$ & $S D$ & $\begin{array}{c}\% \text { in } \\
\text { Compliance }\end{array}$ \\
\hline \multirow{8}{*}{$\begin{array}{l}\text { Refrain from endorsing } \\
\text { prohibited primary cause } \\
\text { of battering }\end{array}$} & & 3.71 & 2.36 & $80 \%$ \\
\hline & Past experience & & & $86 \%$ \\
\hline & Low self-esteem & & & $86 \%$ \\
\hline & Poor impulse control & & & $89 \%$ \\
\hline & Unconscious motivation & & & $89 \%$ \\
\hline & Anger & & & $91 \%$ \\
\hline & $\begin{array}{l}\text { Client or victim mental health } \\
\text { problems }\end{array}$ & & & $91 \%$ \\
\hline & Substance use or abuse & & & $94 \%$ \\
\hline \multirow{9}{*}{$\begin{array}{l}\text { Refrain from prohibited } \\
\text { program curriculum }\end{array}$} & & .23 & .55 & $83 \%$ \\
\hline & Encourage victim or partner disclosure & & & $91 \%$ \\
\hline & $\begin{array}{l}\text { Support or recommend couples/family } \\
\text { counseling or medication }\end{array}$ & & & $91 \%$ \\
\hline & Require victim or partner disclosure & & & $97 \%$ \\
\hline & Use actions of moral superiority & & & $97 \%$ \\
\hline & View battering as addiction & & & $100 \%$ \\
\hline & Encourage ventilation techniques & & & $100 \%$ \\
\hline & Blame battering on victim qualities & & & $100 \%$ \\
\hline & View battering as bi-directional & & & $100 \%$ \\
\hline \multirow{9}{*}{$\begin{array}{l}\text { Approved intervention } \\
\text { strategies }\end{array}$} & & 7.77 & .49 & $80 \%$ \\
\hline & $\begin{array}{l}\text { Increase recognition of criminal aspect } \\
\text { of behavior and thoughts }\end{array}$ & & & $86 \%$ \\
\hline & $\begin{array}{l}\text { Increase understanding of causes, types } \\
\text { and effects of battering }\end{array}$ & & & $97 \%$ \\
\hline & Reinforce personal responsibility & & & $97 \%$ \\
\hline & $\begin{array}{l}\text { Reinforce appropriate beliefs and } \\
\text { behavioral alternatives }\end{array}$ & & & $97 \%$ \\
\hline & Respectful confrontation & & & $100 \%$ \\
\hline & Address tactics to justify & & & $100 \%$ \\
\hline & Stress impact of battering on victims & & & $100 \%$ \\
\hline & $\begin{array}{l}\text { Promote accountability for controlling } \\
\text { and abusive behavior }\end{array}$ & & & $100 \%$ \\
\hline
\end{tabular}


Table 7.

Compliance with Policies Relating to Victims and Partners

\begin{tabular}{|c|c|c|c|c|c|}
\hline Standards Requirement & Sub-Requirements & $n$ & $M$ & $S D$ & $\begin{array}{c}\% \text { in } \\
\text { Compliance }\end{array}$ \\
\hline $\begin{array}{l}\text { Victim information only } \\
\text { available to designated BIP } \\
\text { staff }\end{array}$ & & 34 & & & $79 \%$ \\
\hline \multirow[t]{3}{*}{$\begin{array}{l}\text { Refrain from prohibited } \\
\text { victim contact }\end{array}$} & & 29 & & & $69 \%$ \\
\hline & Solicit information & 29 & & & $69 \%$ \\
\hline & $\begin{array}{l}\text { Informing victim about } \\
\text { things client said in } \\
\text { group }\end{array}$ & 29 & & & $97 \%$ \\
\hline $\begin{array}{l}\text { Distribute informational } \\
\text { materials to victims }\end{array}$ & & 34 & & & $74 \%$ \\
\hline \multirow[t]{7}{*}{$\begin{array}{l}\text { Materials include all required } \\
\text { information }\end{array}$} & & 25 & 4.52 & 1.61 & $40 \%$ \\
\hline & $\begin{array}{l}\text { Includes victim } \\
\text { advocacy resources }\end{array}$ & 25 & & & $96 \%$ \\
\hline & $\begin{array}{l}\text { Includes victims' rights } \\
\text { information }\end{array}$ & 25 & & & $68 \%$ \\
\hline & $\begin{array}{l}\text { Includes safety planning } \\
\text { information }\end{array}$ & 25 & & & $64 \%$ \\
\hline & Includes BIP description & 25 & & & $74 \%$ \\
\hline & $\begin{array}{l}\text { Includes statement of } \\
\text { BIP limitations }\end{array}$ & 25 & & & $72 \%$ \\
\hline & $\begin{array}{l}\text { Includes BIP contact } \\
\text { information }\end{array}$ & 25 & & & $76 \%$ \\
\hline
\end{tabular}


Table 8.

Compliance with Community Collaboration Requirements

\begin{tabular}{|l|l|c|c|}
\hline \multicolumn{1}{|c|}{ Standards Requirement } & \multicolumn{1}{|c|}{ Sub-Requirements } & $n$ & $\%$ in Compliance \\
\hline $\begin{array}{l}\text { Contact with victim advocacy } \\
\text { agency }\end{array}$ & & 35 & $91 \%$ \\
\hline & Designated liaison & 32 & $91 \%$ \\
\hline $\begin{array}{l}\text { Staff member attends DV } \\
\text { council meetings }\end{array}$ & $\begin{array}{l}\text { Submit policies and } \\
\text { procedures }\end{array}$ & 32 & $69 \%$ \\
\hline Contact with LSA & 30 & $87 \%$ \\
\hline $\begin{array}{l}\text { Liaison to criminal justice } \\
\text { system }\end{array}$ & 35 & $71 \%$ \\
\hline & $\begin{array}{l}\text { Communicate program } \\
\text { outcomes }\end{array}$ & 33 & $94 \%$ \\
\hline Collaboration with BIPs & Communicate attendance & 33 & $70 \%$ \\
\hline & & 35 & $94 \%$ \\
\hline Collaboration in the community & $\begin{array}{l}\text { Participation in local or } \\
\text { statewide BIP }\end{array}$ & 35 & $43 \%$ \\
\hline
\end{tabular}


Table 9.

Overview of Qualitative Codes

\begin{tabular}{|c|c|c|c|c|c|}
\hline Construct & Code & Frequency & $\begin{array}{c}\% \\
\text { Mentioned }\end{array}$ & $M(S D)$ & Exemplar Quote \\
\hline \multirow{3}{*}{ Actual Control } & $\begin{array}{l}\text { High Actual } \\
\text { Control }\end{array}$ & 46 & $85 \%$ & $\begin{array}{c}3.54 \\
(S D=3.41)\end{array}$ & $\begin{array}{l}\text { "I think somebody nominated me because I kept } \\
\text { attending and being involved... and then... I was } \\
\text { voted on as a member" }\end{array}$ \\
\hline & $\begin{array}{l}\text { Low Actual } \\
\text { Control }\end{array}$ & 30 & $77 \%$ & $\begin{array}{c}2.31 \\
(S D=1.60)\end{array}$ & $\begin{array}{l}\text { "I know nothing. I don't know when, I don't know } \\
\text { how, I don't know why" }\end{array}$ \\
\hline & $\begin{array}{l}\text { Actual Control } \\
\text { Ratio }\end{array}$ & - & - & $\begin{array}{c}.46 \\
(S D=.52) \\
\end{array}$ & - \\
\hline \multirow{5}{*}{ Perceived Control } & $\begin{array}{l}\text { High Perceived } \\
\text { Ability }\end{array}$ & 18 & $62 \%$ & $\begin{array}{c}1.38 \\
(S D=1.45)\end{array}$ & $\begin{array}{l}\text { "I think that if I were to be involved in the re- } \\
\text { creation of this, my input would be considered and } \\
\text { there would be some adjustments made as a result } \\
\text { of my input. So I do think that my voice would be } \\
\text { heard..." }\end{array}$ \\
\hline & $\begin{array}{l}\text { Low Perceived } \\
\text { Ability }\end{array}$ & 21 & $77 \%$ & $\begin{array}{c}1.62 \\
(S D=1.66)\end{array}$ & $\begin{array}{l}\text { "A lot of politics has entered in. I'm willing to } \\
\text { discuss anything with anybody but I don't want to } \\
\text { discuss it. I don't want to be yelled and screamed at. } \\
\text { And I don't want to be dismissed" }\end{array}$ \\
\hline & $\begin{array}{l}\text { High } \\
\text { Procedural Justice }\end{array}$ & 12 & $38 \%$ & $\begin{array}{c}.92 \\
(S D=1.55)\end{array}$ & $\begin{array}{l}\text { "I think for the most part I've been really } \\
\text { appreciative... I think [the committee] has done a } \\
\text { really good job of hearing us" }\end{array}$ \\
\hline & $\begin{array}{l}\text { Low Procedural } \\
\text { Justice }\end{array}$ & 32 & $69 \%$ & $\begin{array}{c}2.46 \\
(S D=2.54)\end{array}$ & "It's majority culture, middle class, professionals" \\
\hline & $\begin{array}{l}\text { Perceived Control } \\
\text { Ratio }\end{array}$ & - & - & $\begin{array}{c}.34 \\
(S D=.29)\end{array}$ & - \\
\hline
\end{tabular}




\begin{tabular}{|c|c|c|c|c|c|}
\hline Construct & Code & Frequency & $\begin{array}{c}\% \\
\text { Mentioned } \\
\end{array}$ & $M(S D)$ & Exemplar Quote \\
\hline \multirow{6}{*}{$\begin{array}{l}\text { Negative Attitude } \\
\text { Change and } \\
\text { Maintenance }\end{array}$} & $\begin{array}{l}\text { Positive Initial } \\
\text { Response }\end{array}$ & 36 & $92 \%$ & $\begin{array}{c}2.77 \\
(S D=2.17)\end{array}$ & $\begin{array}{l}\text { "I felt like it was progress... for batterers } \\
\text { intervention in Oregon" }\end{array}$ \\
\hline & $\begin{array}{l}\text { Negative Initial } \\
\text { Response }\end{array}$ & 35 & $85 \%$ & $\begin{array}{c}2.69 \\
(S D=1.55)\end{array}$ & $\begin{array}{l}\text { "I thought it was a mistake. We don't know } \\
\text { enough" }\end{array}$ \\
\hline & $\begin{array}{l}\text { Initial Response } \\
\text { Ratio }\end{array}$ & - & - & $\begin{array}{c}.52 \\
(S D=.31)\end{array}$ & - \\
\hline & $\begin{array}{l}\text { Positive Current } \\
\text { Response }\end{array}$ & 116 & $100 \%$ & $\begin{array}{c}8.92 \\
(S D=2.47)\end{array}$ & $\begin{array}{l}\text { "I think it's important to have some kind of uniform } \\
\text { criteria for these kinds of programs" }\end{array}$ \\
\hline & $\begin{array}{l}\text { Negative Current } \\
\text { Response }\end{array}$ & 179 & $100 \%$ & $\begin{array}{c}13.77 \\
(S D=10.19)\end{array}$ & $\begin{array}{l}\text { "The approach to standards is very simplistic and I } \\
\text { think that the problem and the change of the } \\
\text { problem is a... much more complex answer" }\end{array}$ \\
\hline & $\begin{array}{l}\text { Current Response } \\
\text { Ratio }\end{array}$ & - & - & $\begin{array}{c}.44 \\
(S D=.15)\end{array}$ & - \\
\hline \multirow{3}{*}{ Absoluteness } & Absoluteness & 77 & $92 \%$ & $\begin{array}{c}5.92 \\
(S D=6.82)\end{array}$ & $\begin{array}{l}\text { "We all know if we don't comply to these standards } \\
\text { then we don't get referrals" }\end{array}$ \\
\hline & Non-absoluteness & 48 & $85 \%$ & $\begin{array}{c}3.69 \\
(S D=3.11)\end{array}$ & $\begin{array}{l}\text { "I don't think there are any [consequences]. That's } \\
\text { my understanding. Maybe I'm not right but to my } \\
\text { knowledge, nothing" }\end{array}$ \\
\hline & Absoluteness Ratio & - & - & $\begin{array}{c}.58 \\
(S D=.25)\end{array}$ & - \\
\hline
\end{tabular}




\begin{tabular}{|c|c|c|c|c|c|}
\hline Construct & Code & Frequency & $\begin{array}{c}\% \\
\text { Mentioned }\end{array}$ & $M(S D)$ & Exemplar Quote \\
\hline \multirow{10}{*}{ Legitimacy } & $\begin{array}{l}\text { High Procedural } \\
\text { Justice }\end{array}$ & 12 & $38 \%$ & $\begin{array}{c}.92 \\
(S D=1.55)\end{array}$ & $\begin{array}{l}\text { "I think for the most part I've been really } \\
\text { appreciative... I think [the committee] has done a } \\
\text { really good job of hearing us" }\end{array}$ \\
\hline & $\begin{array}{l}\text { Low Procedural } \\
\text { Justice }\end{array}$ & 32 & $69 \%$ & $\begin{array}{c}2.46 \\
(S D=2.54)\end{array}$ & "It's majority culture, middle class, professionals" \\
\hline & $\begin{array}{l}\text { Procedural Justice } \\
\text { Ratio }\end{array}$ & - & - & $\begin{array}{c}.30 \\
(S D=.25)\end{array}$ & - \\
\hline & Positive Norms & 8 & $46 \%$ & $\begin{array}{c}.62 \\
(S D=.77)\end{array}$ & $\begin{array}{l}\text { "What I've seen is that everybody's pretty on board } \\
\text { with it" }\end{array}$ \\
\hline & $\begin{array}{l}\text { Negative } \\
\text { Norms }\end{array}$ & 12 & $38 \%$ & $\begin{array}{c}.92 \\
(S D=1.38)\end{array}$ & $\begin{array}{l}\text { "I really don't know anybody who is in favor of the } \\
\text { standards the way they are written" }\end{array}$ \\
\hline & Norms Ratio & - & - & $\begin{array}{c}.47 \\
(S D=.39)\end{array}$ & - \\
\hline & High Policy Logic & 20 & $77 \%$ & $\begin{array}{c}1.54 \\
(S D=1.33)\end{array}$ & $\begin{array}{l}\text { "[The standards are] based on the evidence that is } \\
\text { available for the population" }\end{array}$ \\
\hline & Low Policy Logic & 25 & $77 \%$ & $\begin{array}{c}1.92 \\
(S D=2.43)\end{array}$ & $\begin{array}{l}\text { "I don't think they are best practice because I don't } \\
\text { think that there really was a best practice." }\end{array}$ \\
\hline & Policy Logic Ratio & - & - & $\begin{array}{c}.48 \\
(S D=.31)\end{array}$ & - \\
\hline & Legitimacy Ratio & - & - & $\begin{array}{c}.40 \\
(S D=.24)\end{array}$ & - \\
\hline
\end{tabular}




\begin{tabular}{|c|c|c|c|c|c|}
\hline Construct & Code & Frequency & $\begin{array}{c}\% \\
\text { Mentioned }\end{array}$ & $M(S D)$ & Exemplar Quote \\
\hline \multirow{6}{*}{ Implementation } & $\begin{array}{l}\text { Implementation } \\
\text { Strategies }\end{array}$ & 57 & $92 \%$ & $\begin{array}{c}4.38 \\
(S D=3.04)\end{array}$ & $\begin{array}{l}\text { "Obviously there were curriculum adjustments, } \\
\text { policy adjustments, procedure adjustments" }\end{array}$ \\
\hline & $\begin{array}{l}\text { Implementation } \\
\text { Ease }\end{array}$ & 67 & $92 \%$ & $\begin{array}{c}5.15 \\
(S D=3.58)\end{array}$ & $\begin{array}{l}\text { "Most... everything that the standards require we } \\
\text { had in place before the standards came out, so it } \\
\text { wasn't really a challenge" }\end{array}$ \\
\hline & $\begin{array}{l}\text { Implementation } \\
\text { Difficulty }\end{array}$ & 69 & $100 \%$ & $\begin{array}{c}5.31 \\
(S D=3.15)\end{array}$ & $\begin{array}{l}\text { "Getting training hours [is most difficult]. It's } \\
\text { because some of the training is unavailable. That's } \\
\text { been the number one thing." }\end{array}$ \\
\hline & Enablers & 53 & $85 \%$ & $\begin{array}{c}4.08 \\
(S D=3.52)\end{array}$ & $\begin{array}{l}\text { "Corrections... I mean just the fact that they'll even } \\
\text { give me the proposed standards and talk to me } \\
\text { about it is great. I'd call that support" }\end{array}$ \\
\hline & Barriers & 105 & $100 \%$ & $\begin{array}{c}8.08 \\
(S D=5.12)\end{array}$ & $\begin{array}{l}\text { "[A barrier is] making sure that all of our } \\
\text { facilitators are getting the training they need, even } \\
\text { though there is some training that is really not } \\
\text { available out there, so we can't do it all" }\end{array}$ \\
\hline & Needed Support & 68 & $100 \%$ & $\begin{array}{c}5.23 \\
(S D=3.42)\end{array}$ & $\begin{array}{l}\text { "I think... the connected communication between } \\
\text { different BIP facilitators and BIP facilities could } \\
\text { help us all. Just having regular conferences on } \\
\text { occasion to talk about our different programs and } \\
\text { how we're complying with the standards and OARs } \\
\text { and everything. I think it would be helpful across } \\
\text { the state" }\end{array}$ \\
\hline
\end{tabular}




\begin{tabular}{|c|c|c|c|c|c|}
\hline Construct & Code & Frequency & $\begin{array}{c}\% \\
\text { Mentioned } \\
\end{array}$ & $M(S D)$ & Exemplar Quote \\
\hline \multirow{4}{*}{ Former Providers } & High Impact & 8 & $60 \%$ & $\begin{array}{c}1.60 \\
(S D=2.07)\end{array}$ & $\begin{array}{l}\text { "The standards absolutely impacted my program } \\
\text { but it was in combination with the environment" }\end{array}$ \\
\hline & Low Impact & 9 & $100 \%$ & $\begin{array}{c}1.80 \\
(S D=.84)\end{array}$ & $\begin{array}{l}\text { "We had to be part of the DV community because } \\
\text { of the standards and so we had to pay for someone } \\
\text { to got to those meetings. This expense impacted our } \\
\text { finances and finances are why we stopped. But, to } \\
\text { be honest, we would have gone to those meetings } \\
\text { with out the standards. So no, they didn't make us } \\
\text { close at all" }\end{array}$ \\
\hline & Impact Ratio & - & - & $\begin{array}{c}.37 \\
(S D=.38)\end{array}$ & - \\
\hline & Interest & 4 & $60 \%$ & $\begin{array}{c}.80 \\
(S D=.84)\end{array}$ & $\begin{array}{l}\text { "I definitively did not stop because I didn't want to } \\
\text { do the work anymore, I enjoyed the work" }\end{array}$ \\
\hline \multirow{3}{*}{ Other } & $\begin{array}{l}\text { Limited } \\
\text { Contact }\end{array}$ & 6 & $31 \%$ & $\begin{array}{c}.46 \\
(S D=.78)\end{array}$ & $\begin{array}{l}\text { I wish I was more [involved]. I would love to be in } \\
\text { the loop more, but I'm not" }\end{array}$ \\
\hline & Discussion & 30 & $85 \%$ & $\begin{array}{c}2.31 \\
(S D=1.97)\end{array}$ & $\begin{array}{l}\text { "People have a lot of questions about what's in [the } \\
\text { standards]...especially the new people. So } \\
\text { [discussion] is mostly just going over basic things } \\
\text { about what's required or not required, or what I am } \\
\text { supposed to be doing or not doing. It's not about } \\
\text { this is bad or this is good, it's this is what [the } \\
\text { standards include]" }\end{array}$ \\
\hline & $\begin{array}{l}\text { Social Action } \\
\text { Research }\end{array}$ & 26 & $46 \%$ & $\begin{array}{c}2.00 \\
(S D=2.80)\end{array}$ & $\begin{array}{l}\text { "I learned some of the standards just by taking your } \\
\text { survey, it was a good survey" }\end{array}$ \\
\hline
\end{tabular}


Table 10.

Implementation Strategies

\begin{tabular}{|l|c|l|}
\hline \multicolumn{1}{|c|}{ Strategy } & $\begin{array}{c}\% \\
\text { Mentioned }\end{array}$ & \multicolumn{1}{c|}{ Exemplar Quote } \\
\hline $\begin{array}{l}\text { Shifting practices } \\
\text { or policies }\end{array}$ & $54 \%$ & $\begin{array}{l}\text { "Obviously there were curriculum adjustments, policy } \\
\text { adjustments, procedure adjustments" }\end{array}$ \\
\hline Attend trainings & $45 \%$ & $\begin{array}{l}\text { "Even though I have limited resources I pay to have [staff] go out } \\
\text { and get trained" }\end{array}$ \\
\hline Host trainings & $23 \%$ & $\begin{array}{l}\text { "We found it has worked really well to invite victims advocates... } \\
\text { and train them in batterers intervention. We've had some drug and } \\
\text { alcohol counselors who were interested in [batterers intervention] } \\
\text { and we train them on that side of things". }\end{array}$ \\
\hline $\begin{array}{l}\text { Fostering } \\
\text { relationships }\end{array}$ & $38 \%$ & $\begin{array}{l}\text { "I think there's been an attempt to do the collaborative work and } \\
\text { let other people know what I do and how I do what I do" }\end{array}$ \\
\hline Read standards & $38 \%$ & $\begin{array}{l}\text { "I read them thoroughly. I underline things. I make sure that I } \\
\text { understand what they're asking. I take them very seriously" }\end{array}$ \\
\hline Hire staff & $15 \%$ & $\begin{array}{l}\text { "We brought in a(n) [opposite sex] facilitator so that we have co- } \\
\text { facilitation" }\end{array}$ \\
\hline
\end{tabular}


Table 11.

Implementation Ease

\begin{tabular}{|c|c|c|}
\hline $\begin{array}{c}\text { Policy or } \\
\text { Characteristic }\end{array}$ & $\begin{array}{c}\% \\
\text { Mentioned }\end{array}$ & Exemplar Quote \\
\hline Overall ease & $69 \%$ & $\begin{array}{l}\text { "Most... everything that the standards require we had in place } \\
\text { before the standards came out, so it wasn't really a challenge" }\end{array}$ \\
\hline Program length & $69 \%$ & $\begin{array}{l}\text { "Obviously the length was the easiest to understand and to } \\
\text { implement for us..." }\end{array}$ \\
\hline Aftercare & $17 \%$ & $\begin{array}{l}\text { "We were tasked with developing an aftercare program [which was } \\
\text { relatively easy]" }\end{array}$ \\
\hline $\begin{array}{l}\text { Program } \\
\text { philosophy/curri } \\
\text { culum }\end{array}$ & $54 \%$ & $\begin{array}{l}\text { "Content... I mean that's the kind of core stuff that is pretty easy. I } \\
\text { don't know how you run a batterer's program and not be in line } \\
\text { with those standards of the curriculum" }\end{array}$ \\
\hline $\begin{array}{l}\text { Accountability } \\
\text { plan }\end{array}$ & $23 \%$ & $\begin{array}{l}\text { "The accountability plan and helping the guys be in tune with each } \\
\text { other and hold each other accountable. Those things were already in } \\
\text { place" }\end{array}$ \\
\hline $\begin{array}{l}\text { Community } \\
\text { collaboration }\end{array}$ & $38 \%$ & $\begin{array}{l}\text { "It's really easy to develop working relationships with your shelter } \\
\text { and your advocacy programs" }\end{array}$ \\
\hline Training & $23 \%$ & "We were already doing the 40 hours of training for all staff" \\
\hline $\begin{array}{l}\text { Mixed gender } \\
\text { co-facilitation }\end{array}$ & $23 \%$ & "We were already doing co-facilitation" \\
\hline
\end{tabular}


Table 12.

Implementation Difficulty

\begin{tabular}{|l|c|l|}
\hline $\begin{array}{c}\text { Policy or } \\
\text { Characteristic }\end{array}$ & $\begin{array}{c}\% \\
\text { Mentioned }\end{array}$ & \multicolumn{1}{c|}{ Exemplar Quote } \\
\hline Overall difficulty & $15 \%$ & "I think most of the standards are not easily implemented" \\
\hline Training & $54 \%$ & $\begin{array}{l}\text { "Getting training hours [is most difficult]. It's because some of the } \\
\text { training is unavailable. That's been the number one thing" }\end{array}$ \\
\hline $\begin{array}{l}\text { Mixed gender } \\
\text { co-facilitation }\end{array}$ & $54 \%$ & $\begin{array}{l}\text { "Ideally we would like a co-facilitator. Unfortunately, we don't } \\
\text { have the resources in a rural area like this to do that" }\end{array}$ \\
\hline $\begin{array}{l}\text { Program } \\
\text { philosophy/curri } \\
\text { culum }\end{array}$ & $31 \%$ & $\begin{array}{l}\text { "I think politically it's been a difficulty in terms of the } \\
\text { development of our curricula. It's been challenging" }\end{array}$ \\
\hline $\begin{array}{l}\text { Program length } \\
\text { "We were obviously [changing] our 24 week program into a } 48 \\
\text { week program... but we really didn't get very far [with that due to } \\
\text { county standards]" }\end{array}$ & \begin{tabular}{l} 
"Collaboration and working with the community is the hardest" \\
\hline $\begin{array}{l}\text { Community } \\
\text { collaboration }\end{array}$
\end{tabular}$\quad 23 \%$ \\
\hline $\begin{array}{l}\text { Group size } \\
\text { "The standards say 15 people per class... when you have only two } \\
\text { or three people on staff, your class is as big as your class is" }\end{array}$ \\
\hline
\end{tabular}


Table 13.

Enablers to Compliance

\begin{tabular}{|l|c|l|}
\hline \multicolumn{1}{|c|}{ Enabler } & $\begin{array}{c}\% \\
\text { Mentioned }\end{array}$ & \multicolumn{1}{c|}{ Exemplar Quote } \\
\hline $\begin{array}{l}\text { Agency } \\
\text { enablers }\end{array}$ & $62 \%$ & $\begin{array}{l}\text { "The key stakeholders in our community are supportive, you know, } \\
\text { are willing to look at whatever suggestions we're making. If we were } \\
\text { to say something like, well, the standards say it should be this way, } \\
\text { then they would say okay." }\end{array}$ \\
\hline $\begin{array}{l}\text { Activity } \\
\text { enablers }\end{array}$ & $38 \%$ & $\begin{array}{l}\text { "I think in some ways the involvement of PO monitoring... has been } \\
\text { challenging but helpful" }\end{array}$ \\
\hline $\begin{array}{l}\text { Content } \\
\text { enablers }\end{array}$ & $15 \%$ & $\begin{array}{l}\text { "[The standards] made it very easy to put the program together } \\
\text { because there was the teeth of what I needed contained in the } \\
\text { document. I think the expectations were fairly clear of what each } \\
\text { program should contain and how they should go about providing } \\
\text { services." }\end{array}$ \\
\hline $\begin{array}{l}\text { Program } \\
\text { characteristic } \\
\text { enablers }\end{array}$ & $62 \%$ & $\begin{array}{l}\text { "The agency's support [is an enabler]. The agency has been } \\
\text { committed to this since the 90s" }\end{array}$ \\
\hline $\begin{array}{l}\text { Engagement in } \\
\text { research }\end{array}$ & $46 \%$ & $\begin{array}{l}\text { "I learned some of the standards just by taking your survey, it was a } \\
\text { good survey" }\end{array}$ \\
\hline
\end{tabular}


Table 14.

Barriers to Compliance

\begin{tabular}{|l|c|l|}
\hline \multicolumn{1}{|c|}{ Barrier } & $\begin{array}{c}\% \\
\text { Mentioned }\end{array}$ & \multicolumn{1}{c|}{ Exemplar Quote } \\
\hline Agency barriers & $69 \%$ & $\begin{array}{l}\text { "I'm not being collaborated with. I'm the one doing the } \\
\text { collaboration. I'm the one that supports them. They don't particularly } \\
\text { support" }\end{array}$ \\
\hline $\begin{array}{l}\text { Activity } \\
\text { barriers }\end{array}$ & $54 \%$ & $\begin{array}{l}\text { "[A barrier is] making sure that all of our facilitators are getting the } \\
\text { training they need, even though there is some training that is really } \\
\text { not available out there, so we can't do it all" }\end{array}$ \\
\hline Content barriers & $54 \%$ & $\begin{array}{l}\text { "The standards are so complicated. They are so cumbersome. They } \\
\text { are, from my point of view...if they are not already, they are quickly } \\
\text { becoming unworkable" }\end{array}$ \\
\hline $\begin{array}{l}\text { Program } \\
\text { characteristic } \\
\text { barriers }\end{array}$ & $69 \%$ & $\begin{array}{l}\text { "Eastern Oregon itself [is a barrier]. We're kind of forgotten about } \\
\text { out here" }\end{array}$ \\
\hline
\end{tabular}


Table 15.

Support Needed for Compliance

\begin{tabular}{|l|c|l|}
\hline Support Needed & $\begin{array}{c}\% \\
\text { Mentioned }\end{array}$ & \multicolumn{1}{c|}{ Exemplar Quote } \\
\hline $\begin{array}{l}\text { Relationship } \\
\text { building }\end{array}$ & $46 \%$ & $\begin{array}{l}\text { "I think... the connected communication between different BIP } \\
\text { facilitators and BIP facilities could help us all." }\end{array}$ \\
\hline Monitoring & $38 \%$ & $\begin{array}{l}\text { "If I could see the [monitoring] process as being supportive and not } \\
\text { punitive. Not just you will comply but this will be helpful to build a } \\
\text { stronger, better program..." }\end{array}$ \\
\hline $\begin{array}{l}\text { Modified } \\
\text { content of } \\
\text { standards }\end{array}$ & $54 \%$ & $\begin{array}{l}\text { "Taking all the legal mumbo-jumbo out of the standards... Having it } \\
\text { cut and dry. Here's what the batterer needs to do. Little bullet } \\
\text { statements would be nice... If I could understand them better, I } \\
\text { could implement them better" }\end{array}$ \\
\hline Resources & $46 \%$ & $\begin{array}{l}\text { "Maybe creating some online training would be good. That way... } \\
\text { those of us that are busy could fit it in our schedules" }\end{array}$ \\
\hline
\end{tabular}


Table 16.

Comparison of High and Low Compliance Programs

\begin{tabular}{|c|c|c|c|c|c|c|c|}
\hline Construct & $\begin{array}{l}\text { Compliance } \\
\text { group }\end{array}$ & $\begin{array}{c}M \text { Ratio } \\
(S D)\end{array}$ & Range & $t$ & $d f$ & $p$-value & $d$ \\
\hline \multirow{2}{*}{$\begin{array}{l}\text { Actual } \\
\text { control }\end{array}$} & High & $.65(.41)$ & $0.00-1.00$ & \multirow{2}{*}{1.55} & \multirow{2}{*}{11} & \multirow{2}{*}{.15} & \multirow{2}{*}{1.21} \\
\hline & Low & $.36(.22)$ & $0.00-.67$ & & & & \\
\hline \multirow{2}{*}{$\begin{array}{l}\text { Perceived } \\
\text { control }\end{array}$} & High & $.47(.30)$ & $0.00-1.00$ & \multirow{2}{*}{2.01} & \multirow{2}{*}{11} & \multirow{2}{*}{.07} & \multirow{2}{*}{.93} \\
\hline & Low & $.18(.21)$ & $0.00-.43$ & & & & \\
\hline \multirow{2}{*}{ Legitimacy } & High & $.37(.23)$ & $0.00-.67$ & \multirow{2}{*}{-.48} & \multirow{2}{*}{11} & \multirow{2}{*}{.64} & \multirow{2}{*}{.27} \\
\hline & Low & $.44(.28)$ & $.07-.86$ & & & & \\
\hline
\end{tabular}




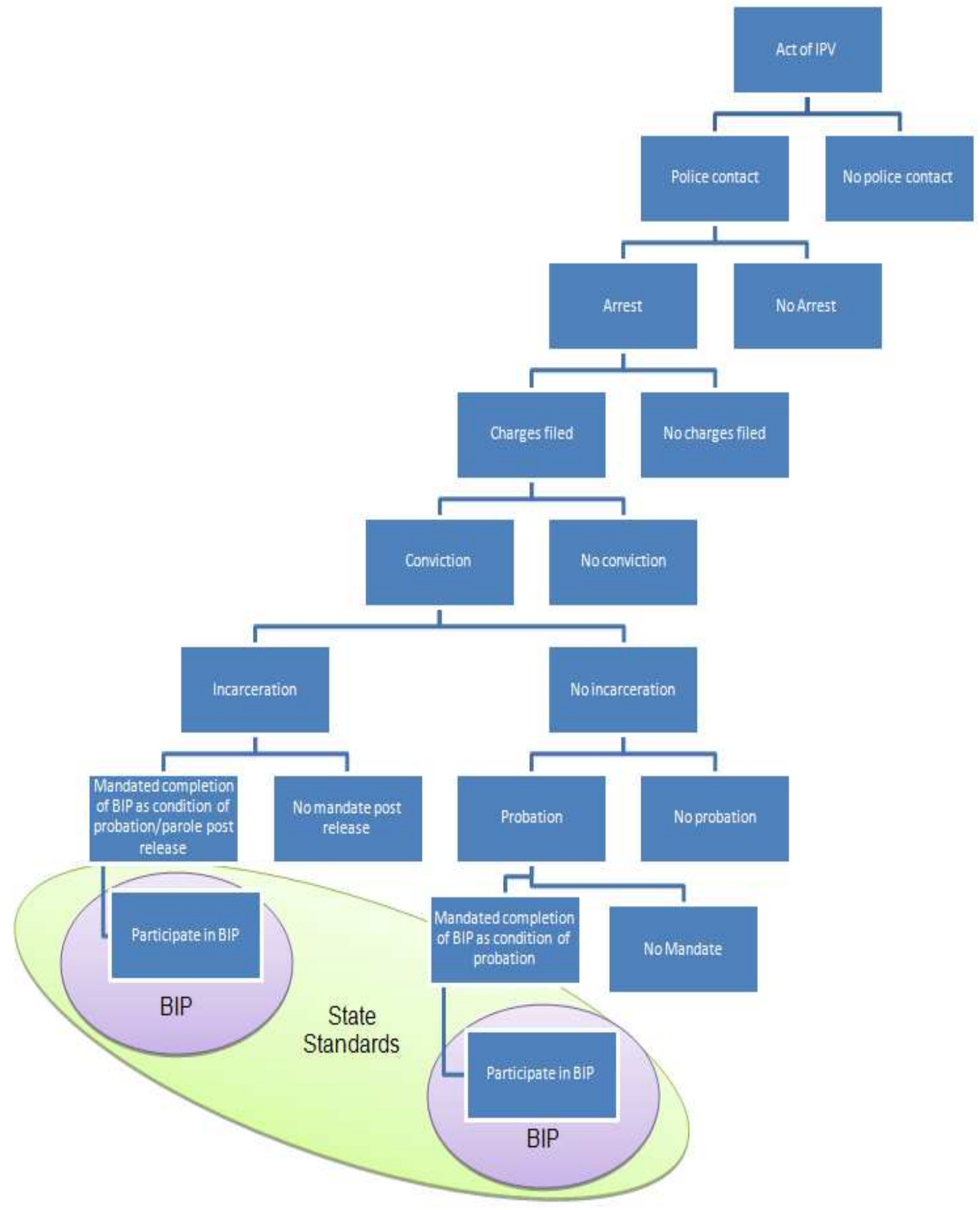

Figure 1. Locating State Standards for BIPs within the Criminal Justice Response to IPV 


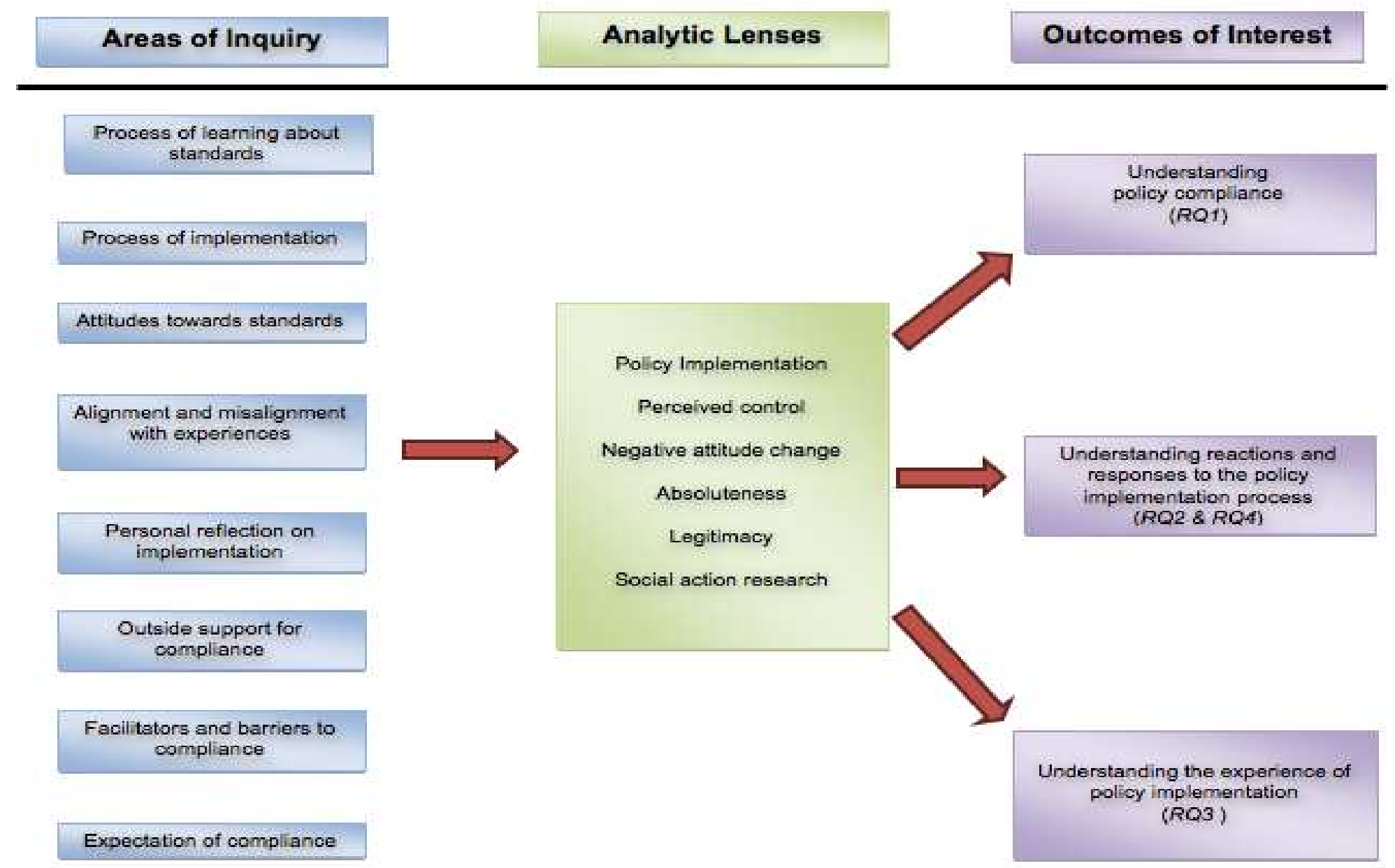

Figure 2. Conceptual Framework: Examining BIP Directors' Response to State Standards 


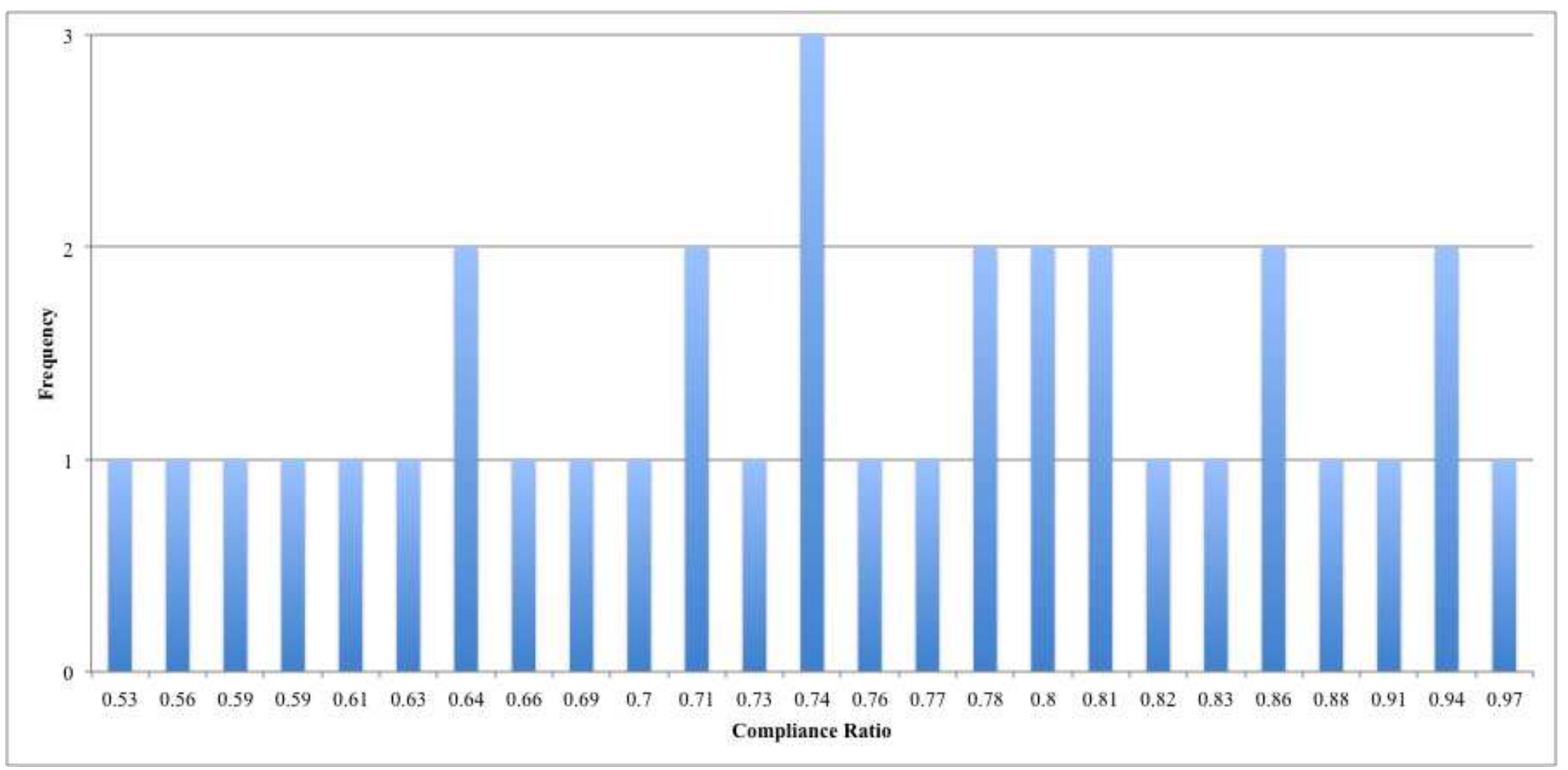

Figure 3. Distribution of compliance ratios $(n=34)$ 


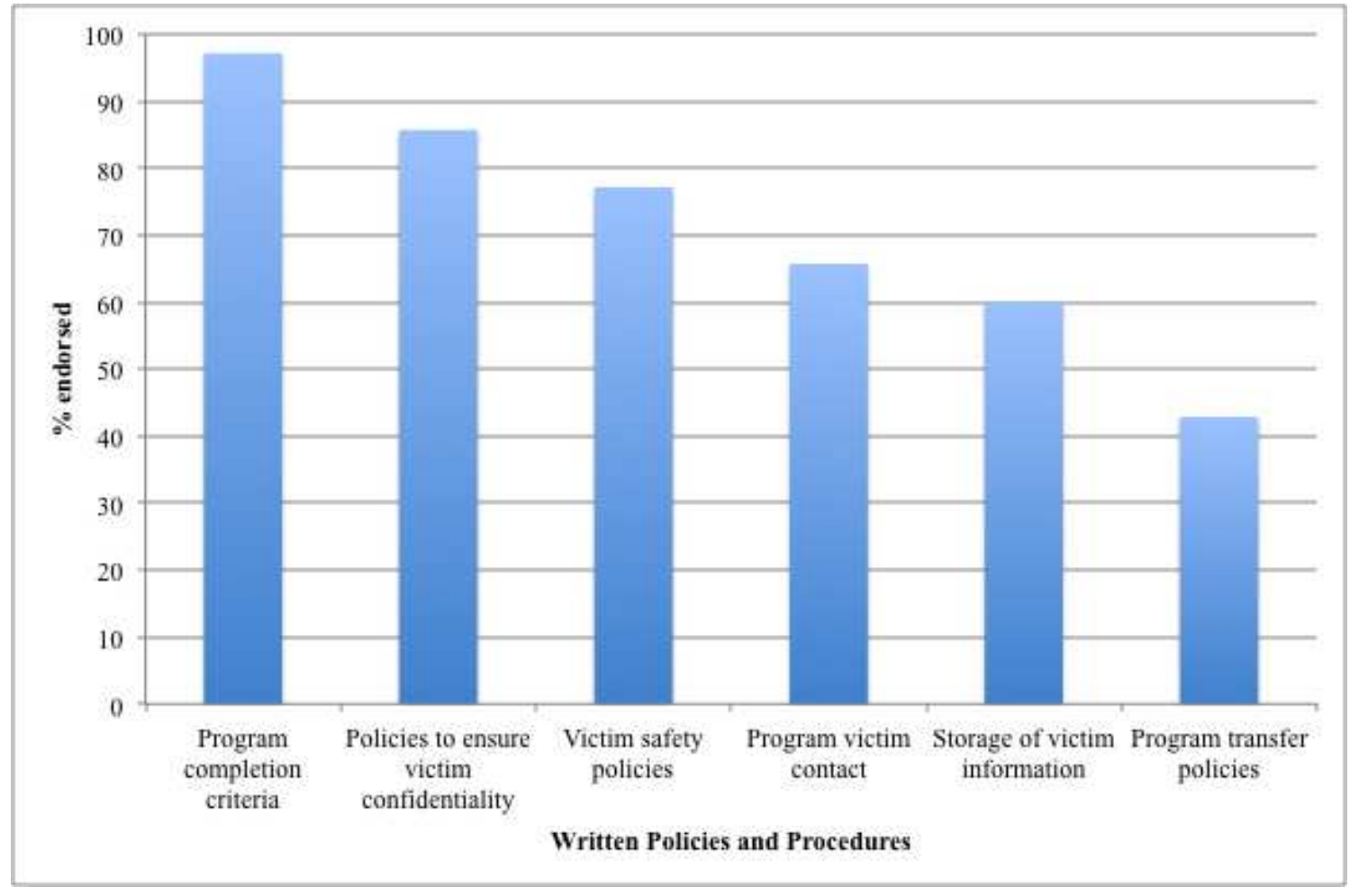

Figure 4. Written policies and procedures $(n=35)$ 


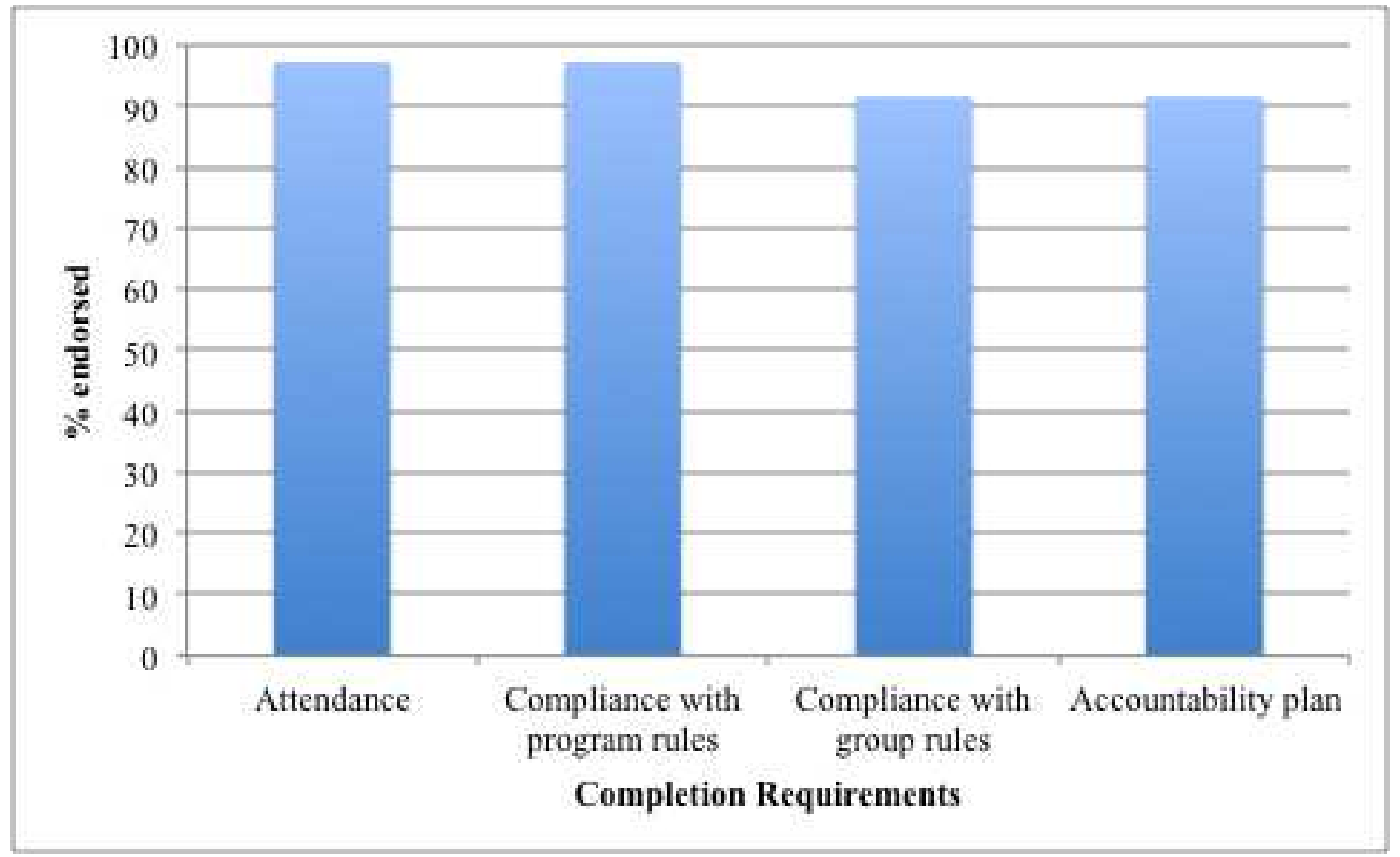

Figure 5. Completion criteria $(n=35)$ 


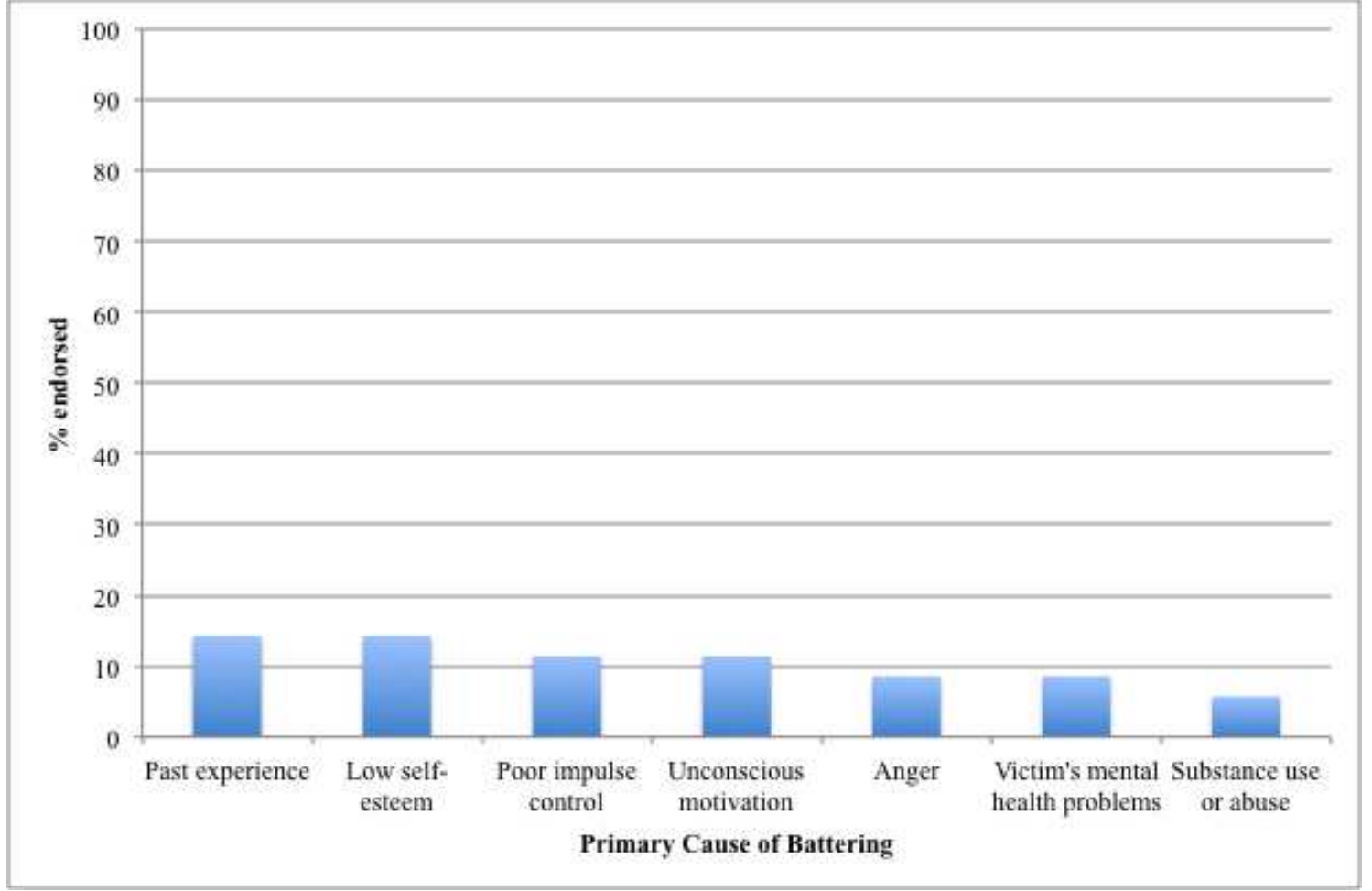

Figure 6. Primary cause of battering $(n=35)$ 


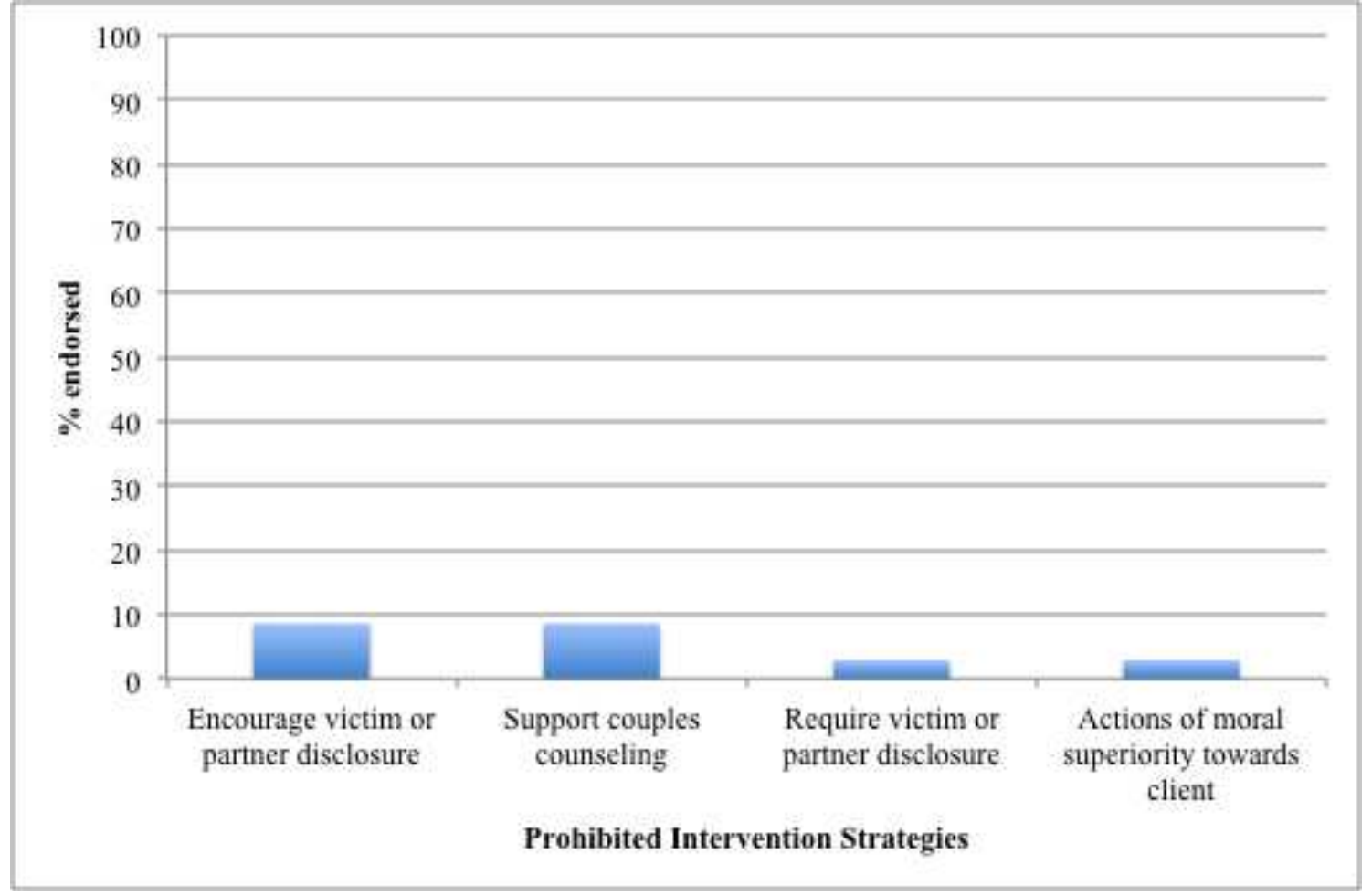

Figure 7. Prohibited intervention strategies $(n=35)$ 


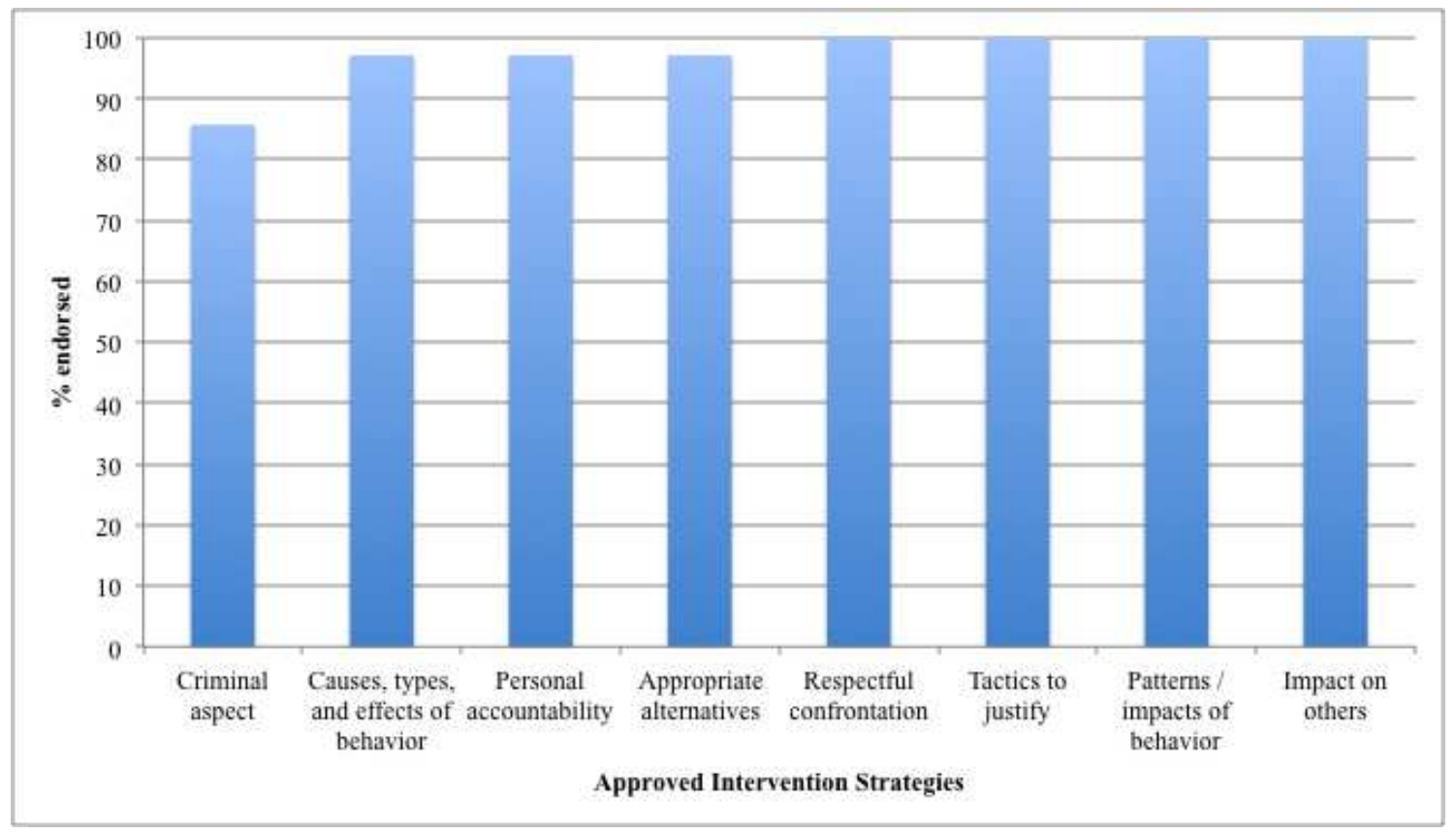

Figure 8. Approved intervention strategies $(n=35)$ 


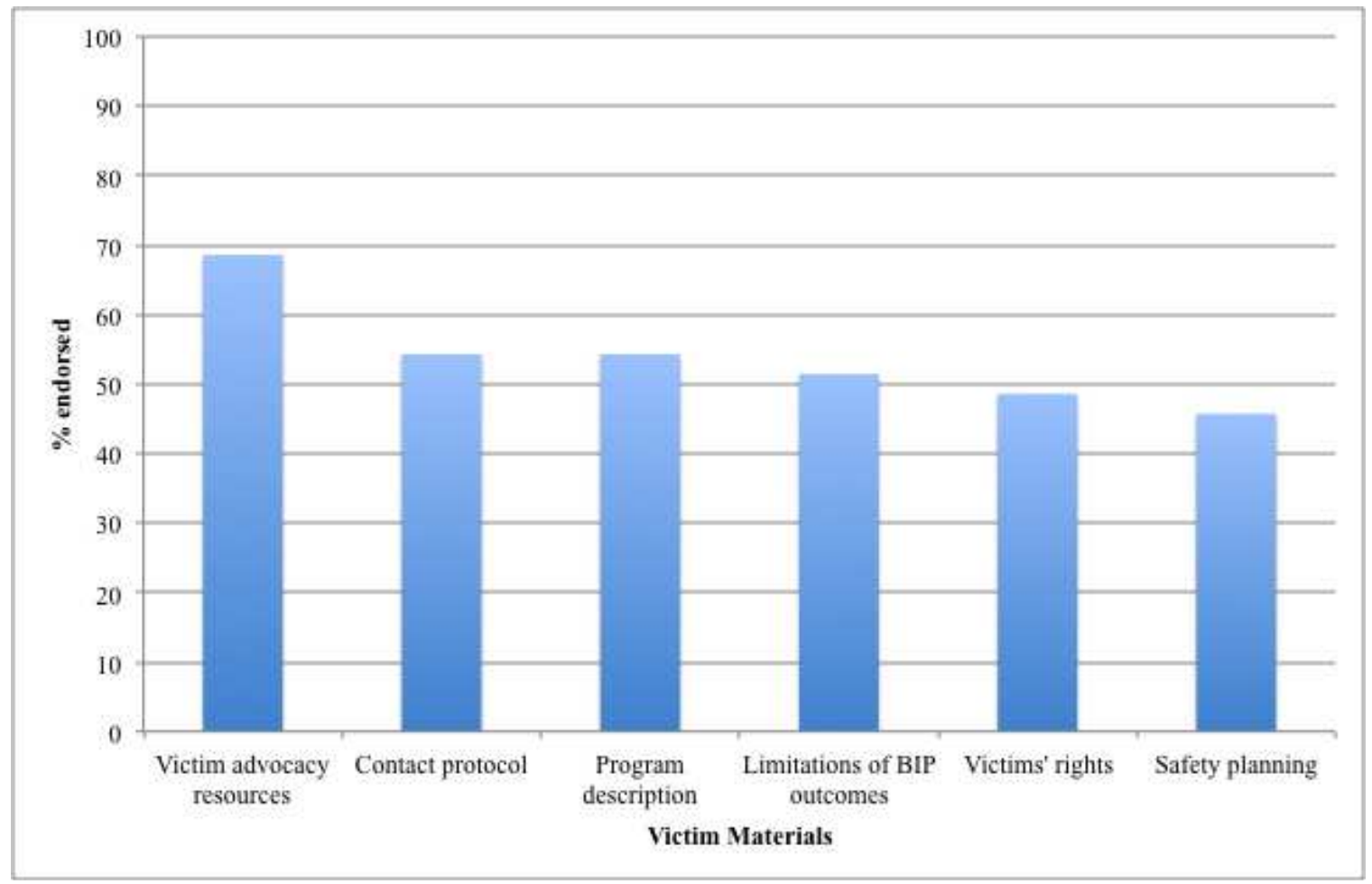

Figure 9. Materials distributed to victims $(n=35)$ 


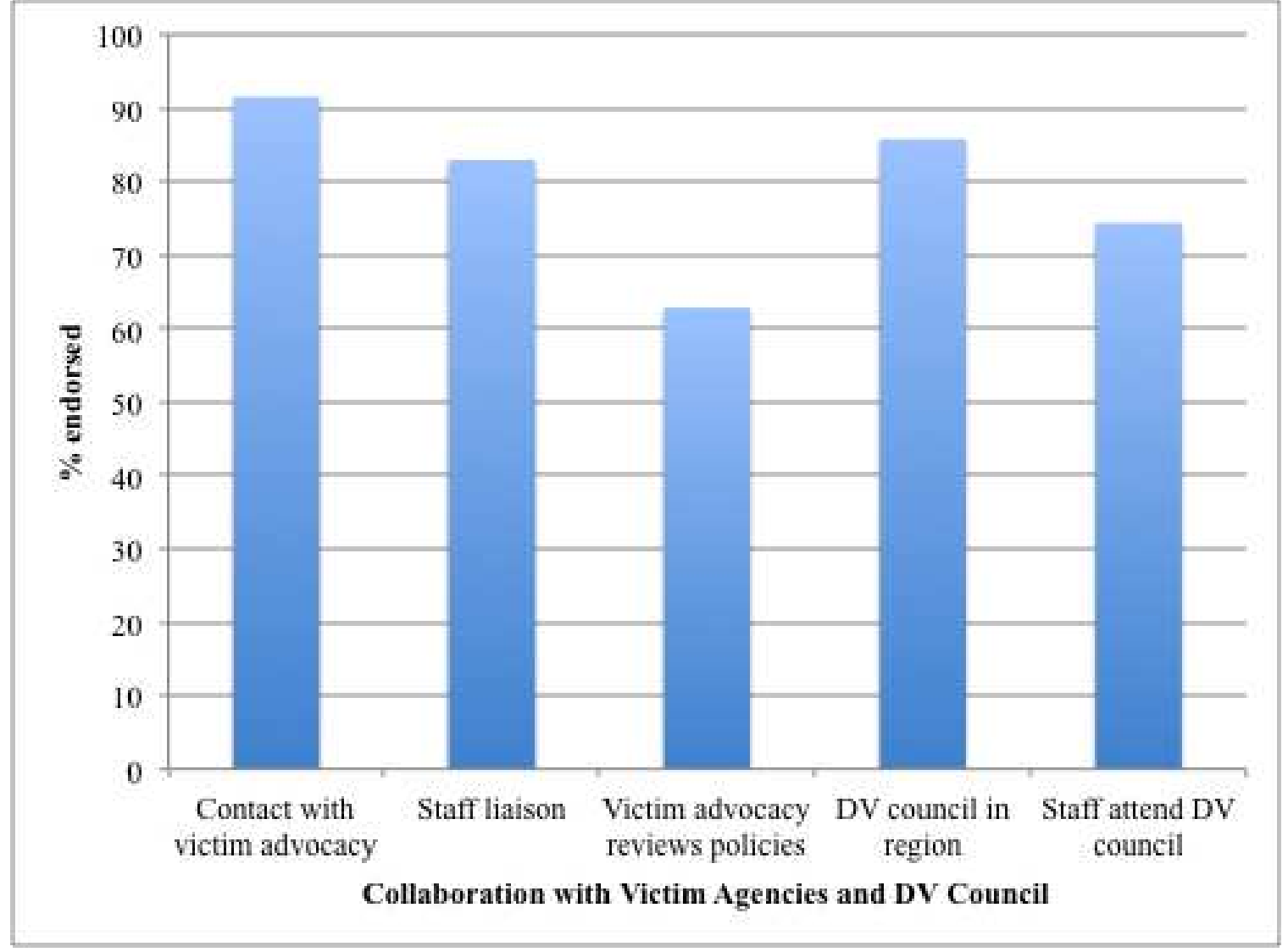

Figure 10. Collaboration with victim advocates and domestic violence council $(n=35)$ 


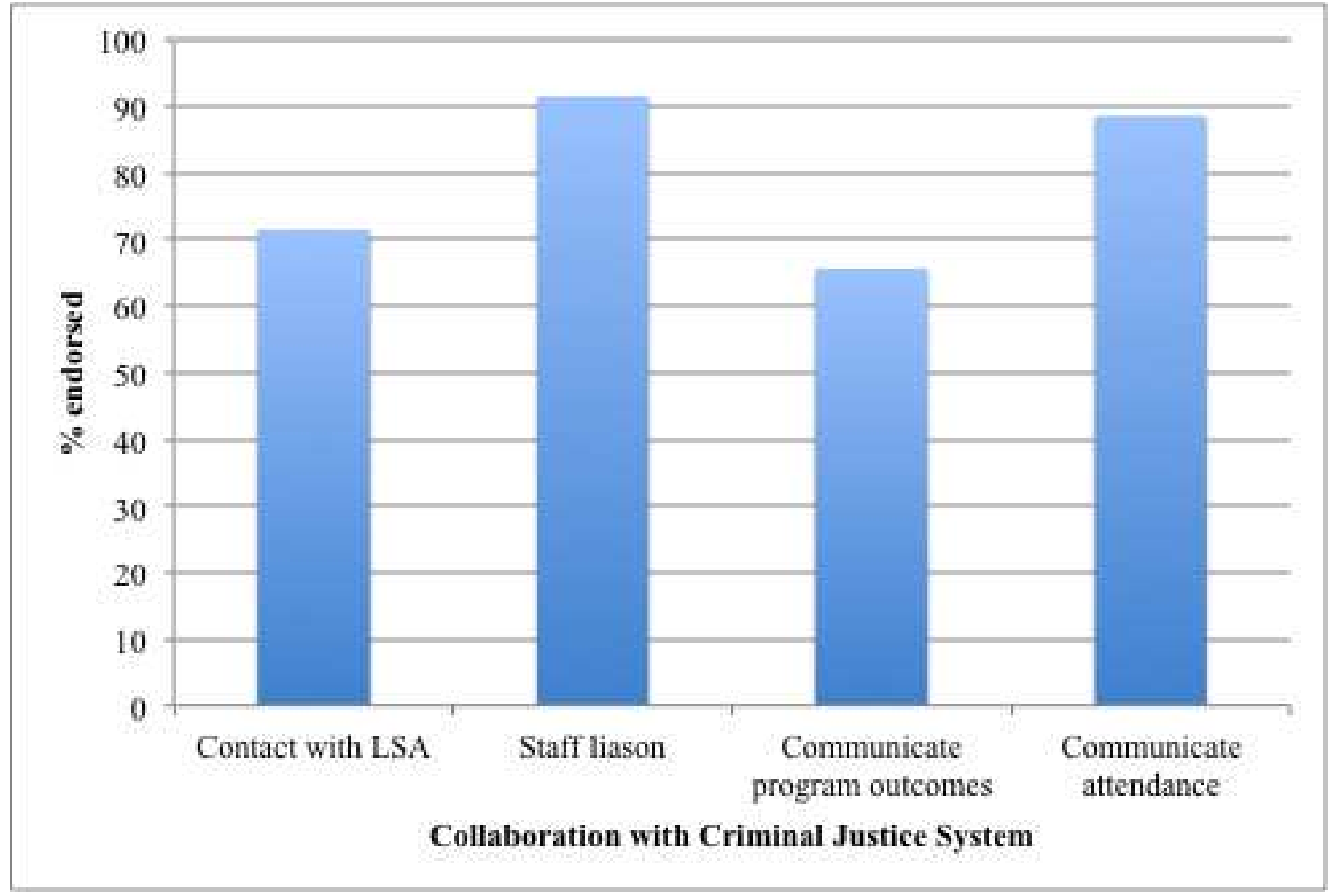

Figure 11. Collaboration with criminal justice system $(n=35)$ 


\section{References}

Adams, D., \& Cayouette, S. (2002). Emerge: A group education model for abusers. In E. Alarondo, \& Mederos, F. (Ed.), Programs for Men Who Batter: Intervention and Prevention Strategies in a Diverse Society. NY: Civic Research Inc.

Adams, J. S., \& Rosenbaum, W. B. (1962). The relationship of worker productivity to cognitive dissonance about wage inequities. Journal of Applied Psychology, 46(3), 161-164.

Adams, A., Soumerai, S. B., Lomas, J., \& Ross-Degnan, D. (1999). Evidence of selfreport bias in assessing adherence to guidelines. International Journal for Quality in Health Care, 187-192.

Ajzen, I., \& Madden, T. J. (1986). Prediction of goal-directed behavior: Attitudes, intentions and perceived behavioral control. Journal of Experimental Social Psychology, 22, 453-474.

Allen, N. E. (2005). A multi-level analysis of community coordinating councils. American Journal of Community Psychology, 35(1/2), 49-63.

Allen, N. E. (2006). An examination of the effectiveness of domestic violence coordinating councils. Violence Against Women, 12, 46-67.

Allen, N. E., Todd, N. R., Anderson, C. J., Davis, S. M., Javandi, S., Bruehleer, V., \& Dorsey, H. (2013). Council-based approaches to intimate partner violence: Evidence for distal change in the system response. American Journal of Community Psychology. Advance online publication. 
Allen, N. E., Watt, K., \& Hess, J. Z. (2008). The outcomes and activities of domestic violence coordinating councils. American Journal of Community Psychology, 41, $63-73$.

Atlas.ti (Version 7) [Computer software]. Berlin, Germany: GmbH.

Austin, J. B., \& Dankwort, J. (1999). Standards for batterer programs: A review and analysis. Journal of Interpersonal Violence, 14(2), 152-168.

Babcock, J. C., Green, C. E., \& Robie, C. (2004). Does batterers' treatment work? A meta-analytic review of domestic violence treatment. Clinical Psychology Review, 23(8), 1023-1053.

Bakeman, R. (2000). Behavioral observation and coding. In H. T. Reis \& C. M. Judd (Eds.), Handbook of Research Methods in Social and Personality Psychology, pp. 138-159. Cambridge, UK: Cambridge University Press.

Bandura, A. (1974). Behavior theory and the models of man. American Psychologist, December 1974, 589-569.

Bandura, A. (1982). Self-efficacy mechanism in human agency. American Psychologist, $37,122-147$.

Banerjee, M., Capozzoli, M., McSweeney, L., \& Sinha, D. (1999). Beyond kappa: A review of interrater agreement measures. The Canadian Journal of Statistics, 27(1), 3-23.

Baron, R., \& Rodin, J. (1978). Perceived control and crowding stress. In A. Baum, J. E. Singer \& S. Valine (Eds.), Advances in Environmental Psychology, pp. 145-190. Hillsdale, NJ: Lawrence Erlbaum Associates. 
Barrett, S. M. (2004). Implementation studies: Time for a revival? Personal reflections on 20 years of implementation studies. Public Administration, 82(2), 249-262.

Barrett-Howard, E., \& Tyler, T. (1986). Procedural justice as a criterion in allocation decisions. Journal of Personality and Social Psychology, 50(2), 296-304.

Bartholomew, K., Henderson, A. J. Z., \& Marcia, J. E. (2000). Coding semi structured interviews in social psychological research. In H. T. Reis \& C. M. Judd (Eds.), Handbook of Research Methods in Social and Personality Psychology, pp. 286312. Cambridge, UK: Cambridge University Press.

Bauer, R. A. (1965). Social psychology and the study of policy formation. Division 8 , American Psychological Association (pp. 933-942). Chicago, IL.

Beasley, R. K., \& Joslyn, M. R. (2001). Cognitive dissonance and post-decision attitude change in six presidential elections. Political Psychology, 22, 521-539.

Becker, A. B., Israel, B. A., Schulz, A. J., Parker, E. A., \& Klem, L. (2002). Predictors of perceived control among African American women in Detroit: Exploring empowerment as a multilevel construct. Health Education \& Behavior, 29, 699715.

Begun, A. L., Shelley, G., Strodthoff, T., \& Short, L. (2001). Adopting a stages of change approach for individuals who are violent with their intimate partners. Journal of Aggression, Maltreatment \& Trauma, 5(2), 105-127.

Bennett, L., \& Piet, M. (1999). Standards for batterer intervention programs: In whose interest? Violence Against Women, 5(6), 6-24. 
Bennett, L., \& Williams, O. (2001). Controversies and recent studies of batterer intervention program effectiveness. [Electronic Version]. Applied Research Forum. Retrieved July 10, 2009, from http://www.vawnet.org

Bennett, L. W., \& Vincent, N. (2001). Standards for batterer programs: A formative evaluation of the Illinois protocol. Journal of Aggression, Maltreatment \& Trauma, 5(2), 181-197.

Berman, P. (1978). Study of macro and micro implementation of social policy. The Rand Paper Series. Retrieved on October 20, 2011, from: www.rand.org/pubs/papers/2008/P6071.pdf

Boal, A. L. (2010). Changes in Oregon Batterer Intervention Program Characteristics in Relation to State Standards (Master's thesis). Available from Dissertation Express database.

Boal, A. L., \& Mankowski, E. S. (in press). Barriers to compliance with Oregon batterer intervention program standards. Violence and Victims.

Bograd, M., \& Mederos, F. (1999). Battering and couples therapy: universal screening and selection of treatment modality. Journal of Marital and Family Therapy, 25(3), 291-312.

Brehm, J. W. (1966). A Theory of Psychological Reactance. New York, New York: Academic Press.

Brehm S. S., \& Brehm, J. W. (1981). Psychological Reactance: A Theory of Freedom and Control. New York, New York: Academic Press. 
Bronfenbrenner, U., \& Morris, P. A. (1998). The ecology of developmental processes. In R. M. Lerner (Ed.), Handbook of Child Psychology, pp. 993-1028. New York, New York: Wiley.

Campbell, J. C., Snow Jones, A., Dienemann, J., Kub, J., Schollenberger, J., O'Campo, P., Carlson Gielen, A., \& Wynne, C. (2002). Intimate partner violence and physical health consequences. Archives of Internal Medicine, 162, 1157-1163.

Catalano, S. (2007). Intimate partner violence in the United States. U.S. Department of Justice: Bureau of Justice Statistics. Retrieved August 5, 2009, from: http://www.ojp.usdoj.gov/bjs/intimate/ipv.htm

Centers for Disease Control and Prevention (2008). Adverse health conditions and health risk behaviors associated with intimate partner violence—United States, 2005. Retrieved October 15, 2011, from: http://www.cdc.gov/mmwr/preview/mmwrhtml/mm5705a1.htm

Centers for Disease Control and Prevention (2010). Intimate Partner Violence: Definitions. Retrieved April 14, 2013, from: http://www.cdc.gov/violenceprevention/intimatepartnerviolence/definitions.html Chai, S., \& Cho, Y. (2011). Cognitions associated with recovery from alcohol dependence. Japanese Psychological Research, 53(3), 327-332.

Cialdini, R. B., \& Goldstein, N. J. (2004). Social influence: Compliance and conformity. Annual Review of Psychology, 55, 591-621. 
Cialdini, R. B., \& Trost, M. R. (1998). Social influence: social norms, conformity, and compliance. In D. T. Gilbert, S. T. Fiske, \& G. Lindzey (Eds.), The Handbook of Social Psychology, (pp. 151-192).Boston, MA, USA: McGraw-Hill.

Cialdini, R. B., Trost, M. R., \& Newsom, J. T. (1995). Preference for consistency: Development of a valid measure and the discovery of surprising behavioral implications. Journal of Personality and Social Psychology, 69(2), 318-328.

Clark, S. J., Burt, M. R., Schulte, M. M., \& Maguire, K. E. (1996). Coordinated community responses to domestic violence in six communities: Beyond the justice system. [Electronic Version], Urban Institute: Research of Record. Retrieved July 15, 2009 from: http://www.urban.org/publications/406727.html

Clee, M. A., \& Wicklund, R. R. (1980). Consumer behavior and psychological reactance. Journal of Consumer Research, 6(4), 389-405.

Cohen, J. (1960). A coefficient of agreement for nominal scales. Educational and Psychological Measurement, 20, 37-46.

Coker, A. L., Davis, K. E., Arias, E., Desai, S., Sanderson, M., Brandt, H. M., \& Smith, P. H. (2002). Physical and mental health effects of intimate partner violence for men and women. American Journal of Preventative Medicine, 23(4), 260-268.

Coker, A. L., Smith, P. H., McKeown, R. E., \& King, M. J. (2000). Frequency and correlates of intimate partner violence by type: Physical, sexual, and psychological battering. American Journal of Public Health, 90(4), 553-559.

Crano, W. D., \& Brewer, M. B. (2002). Principles and Methods of Social Research $\left(2^{\text {nd }}\right.$ ed.). Mahwah, New Jersey: Lawrence Erlbaum Associates. 
Cummings, W. H., \& Venkatesan, M. (1976). Cognitive dissonance and consumer behavior: A review of the evidence. Journal of Marketing Research, 13(3), 303308.

Dalton, B. (2007). What's going on out there? A survey of batterer intervention programs. Journal of Aggression, Maltreatment \& Trauma, 15(1), 59-74.

Dash, D. P. (1999). Current debates in action research. Systemic Practice and Action Research 12(5), 457-492.

Department of Human Services (2013). Domestic Violence. Retrieved April 13, 2013, from: http://www.oregon.gov/dhs/abuse/domestic/Pages/index.aspx

Derzon, J. H., Sale, E., Springer, J. F., \& Brounstein, P. (2005). Estimating intervention effectiveness: Synthetic projection of field evaluation results. The Journal of Primary Prevention, 26, 321-343.

Dickerson, C. A., Thibodeau, R., Aronson, E., \& Miller, D. (1992). Using cognitive dissonance to encourage water conservation. Journal of Applied Social Psychology, 22(11), 841-854.

Dillard, J. P., \& Shen, L. On the nature of reactance and its role in persuasive health communication. Communication Monographs, 72(2), 144-168.

Diver, C. S. (1983). The optimal precision of administrative rules. The Yale Law Journal, 93(1), 65-109.

Donnell, A. J., Thomas, A., \& Buboltz, W. C. (2001). Psychological reactance: Factor structure and internal consistency of the questionnaire for the measurement of psychological reactance. The Journal of Social Psychology, 14(5), 679-687. 
DuBois, D. L., Holloway, B. E., Valentine, J. C., \& Cooper, H. (2002). Effectiveness of mentoring programs for youth: A meta-analytic review. American Journal of Community Psychology, 30, 157-198.

Dufrene, B. A., Noell, G. H., Gilberson, D. N., \& Duhon, G. J. (2005). Monitoring implementation of reciprocal peer tutoring: Identifying and intervening with students who do not maintain accurate implementation. School Psychology Review, 34, 74-86.

Durlak, J. A., \& DuPre, E. P. (2008). Implementation matters: A review of research on the influence of implementation on program outcomes and the factors affecting implementation. American Journal of Community Psychology, 41, 327-350.

Eccles, M. P., Foy, R., Sales, A., Wensing, M., \& Mittman, B. (2012). Implementation Science six years on: Our evolving scope and common reasons for rejection without review. Implementation Science, 7, 71-76.

Elmore, R. F. (1979). Backward mapping: Implementation research and policy decisions. Political Science Quarterly, 94(4), 601-616.

Elo, S., \& Kyngas, H. (2008). The qualitative content analysis process. Journal of Advanced Nursing, 62(1), 107-115.

Erceg-Hurn, D. M., \& Steed, L. G. (2011). Does exposure to cigarette health warning elicit psychological reactance in smokers? Journal of Applied Social Psychology 41(1), 219-237.

Esses, V. M., \& Dovidio, J. F. (2011). Social psychology, social issues, and social policy: What have we learned? Social Issues and Policy Review, 5(1), 1-7. 
Farone, D. W., Fitzpatrick, T. R., \& Bushfield, S. Y. (2008). Hope, locus of control, and quality of health among elder Latina cancer survivors. Social Work in Health Care, 46(2), 51-70.

Feder, L., \& Wilson, D. B. (2005). A meta-analytic review of court-mandated batterer intervention programs: Can courts affect abusers' behavior? Journal of Experimental Criminology, 1, 239-262.

Feder, L., Wilson, D. B., \& Austin, S. (2008). Court-mandated interventions for individuals convicted of domestic violence. Campbell Systematic Reviews, 12, 149.

Federal Bureau of Investigation (2011). Uniform Crime Reports for the United States, 2010.Washington, DC: US Department of Justice, 2011. Retrieved October 15, 2011, from http://www.fbi.gov/about-us/cjis/ucr/crime-in-the-u.s/2010/crime-inthe-u.s.-2010/offenses-known-to-lawenforcement/expanded/expandhomicidemain

Festinger, L. (1957). A theory of cognitive dissonance. Stanford, CA: Stanford University Press.

Festinger, L., \& Carlsmith, J. M. (1959). Cognitive consequences of forced compliance. Journal of Abnormal and Social Psychology, 58, 203-210.

Fine, G. A., \& Elsbach, K. D. (2000). Ethnography and experiment in social psychological theory building: Tactics for integrating qualitative field data with quantitative lab data. Journal of Experimental Social Psychology, 36, 51-76. 
Fischhoff, B. (1990). Psychology and public policy: Tool or toolmaker? American Psychologist, 45 (5), 647-653.

Flouri, E. (2006). Parental interest in children's education, children's self-esteem and locus of control, and later educational attainment: Twenty-six year follow-up of the 1970 British birth cohort. British Journal of Educational Psychology, 76, 4155.

Fontana, A., \& Frey, J. H. (2000). The interview: From structured questions to negotiated text. In N. K. Denzin \& Y. S. Lincoln (Eds.), Handbook of qualitative research ( $2^{\text {nd }}$ ed), pp. 1001-1017. Thousand Oaks, CA: Sage.

Foster-Fishman, P. G., Perkins, D. F., \& Davidson, W. S. (1997). Developing effective evaluation partnerships: Paradigmatic and contextual barriers. Análise Psicólogica, 3, 389-403.

Fraser, J. (1974). Validating a measure of national political legitimacy. American Journal of Political Science, 18(1), 117-134.

French, J. R. P., \& Raven, B. H. (1959). The bases of social power. In D. Cartwright \& A. Zander (Eds.), Group Dynamics, pp. 150-167. New York, NY: Harper \& Row. Fuchs, R. F. (1938). Procedure in administrative rule making. Harvard Law Review, 52 (2), 259-280.

Geffner, R. A., \& Rosenbaum, A. (2001). Domestic violence offenders: Treatment and intervention standards. Journal of Aggression, Maltreatment \& Trauma, 5(2), 1-9.

Gelles, R. J. (2001). Standards for programs for men who batter? Not yet. Journal of Aggression, Maltreatment \& Trauma, 5(2), 11-20. 
Gifford, D., Mianzo, F., \& Briceño-Perriott, J. (2006). Locus of control: Academic achievement and retention in a sample of university first-year students. Journal of College Admission, 18-25.

Gilbert, D.T., Pinel, E.C., Wilson, T.D., Blumberg, S.J., \& Wheatley, T. (1998). Immune neglect: A source of durability bias in affective forecasting. Journal of Personality and Social Psychology, 75, 617-638.

Gist, M. E., Schwoerer, C., \& Rosen, B. (1989). Effects of alternative training methods on self-efficacy and performance in computer software training. Journal of Applied Psychology, 74(6), 884-891.

Golding, J. M. (1999). Intimate partner violence as a risk factor for mental disorders: A meta-analysis. Journal of Family Violence, 14(2), 99-132.

Gondolf, E. W. (1988). The effect of batterer counseling on shelter outcome. Journal of Interpersonal Violence, 3, 275-289.

Gondolf, E. W. (1997). Batterer programs: What we know and need to know. Journal of Interpersonal Violence, 12, 83-98.

Gondolf, E. W. (2002). Batterer Intervention Systems: Issues, Outcomes, and Recommendations. Thousand Oaks: Sage Publications.

Granberg, D., \& Brent, E. (1983). When prophecy bends: The preference-expectation link in U.S. presidential elections, 1952-1980. Journal of Personality and Social Psychology, 45, 477-491. 
Hart, B. J. (1995, March). Coordinated community approaches to domestic violence. Paper presented at the Violence Against Women Research, Strategic Planning Workshop of the National Institute of Justice, Washington DC.

Hettema, J., Steele, J., \& Miller, W. R. (2005). Motivational interviewing. Annual Review of Clinical Psychology, 1, 91-111.

Holden, D. J., Crankshaw, E., Nimsch, C., Hinnant, L. W., \& Hund, L. (2004). Quantifying the impact of participation in local tobacco control groups on psychological empowerment of involved youth. Health Education \& Behavior, $31(5), 615-628$.

Holtzworth-Munroe, A. (2001). Standards for batterer treatment programs: How can research inform our decisions? Journal of Aggression, Maltreatment \& Trauma, 5(2), 165-180.

Hong, S. M. (1992). Hong's psychological reactance scale: A further factor analytic validation. Psychological Reports, 70, 512-514.

Hong, S. M., \& Faedda, S. (1996). Refinement of the Hong psychological reactance scale. Education and Psychological Measurement, 56, 173-182.

Hong, S. M., \& Ostini, R. (1989). Further evaluation of Merz's psychological reactance scale. Psychological Reports, 64, 707-710.

Hsieh, H. F., \& Shannon, S. E. (2005). Three approaches to qualitative content analysis. Qualitative Health Research, 15(9), 1277-1288.

Itzhaky, H., \& York, A. S. (2000). Sociopolitical control and empowerment: An extended replication. Journal of Community Psychology, 28(4), 407-415. 
Jackson, S. (2003). Batterer intervention programs. Batterer intervention programs:

Where do we go from here? Retrieved October 15, 2011, from:

http://nij.gov/pubs-sum/195079.htm

Jellinek, E.M. (1946). Phases in the drinking history of alcoholics. Quarterly Journal of Studies on Alcohol, 7, 1-88.

Kay, A. C., Jimenez, M. C., \& Jost, J. T. (2002).Sour grapes, sweet lemons, and the anticipatory rationalization of the status quo. Personality and Social Psychology Bulletin, 28, 1300-1312.

Kieffer, C. H. (1984). Citizen empowerment: A developmental perspective. Prevention in Human Services, 3, 9-36.

Kish-Gephart, J. J., Harrison, D. A., \&Treviño, L. K. (2010). Bad apples, bad cases, and bad barrels: Meta-analytic evidence about sources of unethical decisions at work. Journal of Applied Psychology, 95(1), 1-31.

Klevens, J., Baker, C. K., Shelley, G. A., \& Ingram, E. M. (2008). Exploring the links between components of coordinated community responses and their impact on contact with intimate partner violence services. Violence Against Women, 14, 346-358.

La Violette, A. (2001). Batterers' treatment: Observations from the trenches. Journal of Aggression, Maltreatment \& Trauma, 5(2), 45-56.

Laurin, K., Kay, A. C., \& Fitzsimons, G. J. (2012). Reactance versus rationalization: Divergent responses to policies that constrain freedom. Psychological Science, 23(2), 205-209. 
Legard, R., Keegan, J., \& Ward, K. (2003). In-depth interviews. In J. Ritchie \& J. Lewis (Eds.), Qualitative research practice: A guide for social science students and researchers, pp. 138-169. Los Angeles, CA: Sage.

Lewin, K. (1946). Action research and minority problems. Journal of Social Issues 2(4), 34-46.

Lincoln, Y. S., \& Guba, E. (1985). Naturalistic Inquiry. Beverly Hills, CA: Sage.

Lindsey, L. L. M. (2005). Anticipated guilt as behavioral motivation: An examination of appeals to help unknown others through bone marrow donation. Human Communication Research, 31(4), 453-481.

Maiuro, R. D., \& Eberle, J. A. (2008). State standards for domestic violence perpetrator treatment: Current status, trends, and recommendations. Violence and Victims, 23(2), 133-155.

Maiuro, R. D., Hagar, T. S., Lin, H., \& Olson, N. (2001). Are current state standards for domestic violence perpetrator treatment adequately informed by research? A question of questions. Journal of Aggression, Maltreatment \& Trauma, 5(2), 2144.

Mann, C. B. (2010). Is there a backlash to social pressure? A large-scale field experiment on voter mobilization. Political Behavior, 32, 387-407.

Max, W., Rice, D. P., Finkelstein, E., Bardwell, R. A., \& Leadbetter, S. (2004). The economic toll of intimate partner violence against women in the workplace. Violence and Victims, 19(3), 259-272. 
Matland, R. E. (1995). Synthesizing the implementation literature: The ambiguityconflict model of policy implementation, Journal of Public Administration Research and Theory, 5(2), 145-174.

Matz, D. C., \& Wood, W. (2005). Cognitive dissonance in groups: The consequences of disagreement. Journal of Personality and Social Psychology, 88(1), 22-37.

Mavropoulou, S., \& Padeliadu, S. (2002). Teachers' casual attributions for behaviour problems in relation to perceptions of control. Educational Psychology, 22(2), 191-202.

Mazis, M. B. (1975). Antipollution measures and psychological reactance: A field experiment. Journal of Personality and Social Psychology, 31 (4), 654-660.

McLaughlin, M. W. (1987). Learning from experience: Lessons from policy implementation. Educational Evaluation and Policy Analysis, 9(2), 171-178.

Mederos, F., \& Perilla, J. (May 2004). Community connections: Men, gender and violence. Paper presented at The Melissa Institute For Violence Prevention and Treatment, $8^{\text {th }}$ Annual Conference, Miami, FL.

Merrick, E. (1999). An exploration of quality in qualitative research: Are "reliability" and “validity” relevant? In M. Kopola \& A. Suzuki (Eds.), Using qualitative methods in psychology, pp. 25-36. London: Sage.

Merz, J. (1983). A questionnaire for the measurement of psychological reactance [in German]. Diagnostica, 29, 75-82. 
Meyers, D. C., Durlak, J. A., \& Wandersman, A. (2012). The quality implementation framework: A synthesis of critical steps in the implementation process. American Journal of Community Psychology, 50, 462-480.

Mondak, J. J. (1994). Policy legitimacy and the Supreme Court: the sources and contexts of legitimation. Political Research Quarterly, 47(3), 675-692.

Morgan, D. L. (1993). Qualitative content analysis: A guide to paths not taken. Qualitative Health Research, 3(1), 112-121.

Morgan, D. L. (1996). Focus groups. Annual Review of Sociology, 22, 129-152.

Morgan, D. L. (1997). Focus Groups as Qualitative Research. Thousand Oaks, CA; Sage Publications.

Morgan, D. L. (1998). Practical strategies for combining qualitative and quantitative methods: Application to health research. Qualitative Health Research, 8, 362-376.

Nail, P. R., \& Boniecki, K. A. (2011). Inconsistency in cognition: Cognitive Dissonance. In D. Chadee (Ed.), Theories in Social Psychology, pp. 44-71, West Sussex, United Kingdom: Blackwell Publishing, Ltd.

National Institutes of Health (2013). Dissemination and implementation. Retrieved July 27, 2013, from: http://obssr.od.nih.gov/scientific_areas/translation/dissemination_and_implement ation/index.aspx 
O’Hea, E. L., Moon, S., Grothe, K. B., Boudreax, E., Bodenlos, J. S., Wallston, K., \& Brantley, P. J. (2009). The interaction of locus of control, self-efficacy, and outcome expectancy in relation to HbA1c in medically underserved individuals with type 2 diabetes. Journal of Behavioral Medicine, 32, 106-117.

O’Leary, K. D. (2001). Conjoint therapy for partners who engage in physically aggressive behavior: Rationale and research. Journal of Aggression, Maltreatment \& Trauma, 5(2), 145-164.

O'Leary, K. D., Heyman, R. E., \& Neidig, P. H. (1999). Treatment of wife abuse: A comparison of gender-specific and conjoint approaches. Behavior Therapy, 30(3), 475-505.

O’Toole, L. J. (1986). Policy recommendations for multi-actor implementation: An assessment of the field. Journal of public policy, 6(2), 181-210.

O’Toole, L. J. (2000). Research on policy implementation: Assessment and prospects. Journal of Public Administration Research and Theory, 10(2), 263-288.

O'Toole, L. J. (2004). The theory-practice issue in policy implementation research. Public Administration, 82(2), 309-329.

Ohmer, M. L. (2008). The relationship between citizen participation and organizational processes and outcomes and the benefits of citizen participation in neighborhood organizations. Journal of Social Service Research, 34(4), 41-60.

Oregon Department of Justice (2009). Batterer intervention program rules. Oregon Administrative Rules. Retrieved May 24, 2009, from: http://arcweb.sos.state.or.us/rules/OARS_100/OAR_137/137_087.html 
Oregon Department of Justice (2009). Batterer Intervention Program (BIP) Advisory Committee. Retrieved April, 2013 from: http://arcweb.sos.state.or.us/rules/OARS_100/OAR_137/137_087.html Paulhus, D. (1983). Sphere-specific measures of perceived control. Journal of Personality and Social Psychology, 44(6), 1253-1265.

Paulhus, D., \& Christie, R. (1981). Spheres of control: An interactionist approach to the assessment of perceived control. In H. M. Lefcourt (Ed), Research within the locus of control construct: Volume 1 - Assessment methods, pp. 161-188. New York, NY: Academic Press, Inc.

Paulhus, D. L., \& Van Selst, M. (1990). The spheres of control scale: 10 yr of research. Personality and Individual Differences, 11(10), 1029-1036.

Price, B. J., \& Rosenbaum, A. (2009). Batterer intervention programs: A report from the field. Violence and Victims, 24(6), 757-770.

Quick, B. L. (2012). What is the best measure of psychological reactance? An empirical test of two measures. Health Communication, 27, 1-9.

Rappaport, J. (1998). The art of social change: Community narratives as resources for individual and collective identity. In X. B. Arriaga \& S. Oskamp (Eds.), Addressing community problems: Psychological research and intervention, pp. 225-246. Thousand Oaks, CA: Sage.

Reeves, C., \& O'Leary-Kelly, A. M. (2007). The effects and costs of intimate partner violence for work organizations. Journal of Interpersonal Violence, 22(3), 327344. 
Ritchie, J., Lewis, J., \& Elam, G. (2003). Designing and selecting samples. In J.

Ritchie \& J. Lewis (Eds.), Qualitative research practice: A guide for social science students and researchers, pp. 77-108. Los Angeles, CA: Sage.

Ritchie, J., Spencer, L., \& O’Connor, W. (2003). Carrying out Qualitative Analysis. In J. Ritchie \& J. Lewis (Eds.), Qualitative research practice: A guide for social science students and researchers, pp. 219-262. Los Angeles, CA: Sage.

Rodin, J. (1990). Control by any other name: Definitions, concepts and processes. In J. Rodin, C. Schooler \& K. W. Schaie (Eds.), Self-Directedness: Cause and Effects Throughout the Life Course, pp. 1-17. Hillsdale, New Jersey: Lawrence Erlbaum Associates.

Rosenbaum, A., \& Leisring, P. A. (2001). Group interventions programs for batterers. Journal of Aggression, Maltreatment \& Trauma, 5(2), 57-71.

Rothbaum, F., Weisz, J. R., \& Snyder, S. S. (1982). Changing the world and changing the self: A two-process model of perceived control. Journal of Personality and Social Psychology, 42(1), 5-37.

Rotter, J. B. (1966). Generalized expectancies for internal versus external control of reinforcement. Psychological Monographs, 80, 1-28.

Roy, V., Lindsay, J., \& Dallaire, L. F. (2012). Mixed-gender co-facilitation in therapeutic groups for men who have perpetrated intimate partner violence: Group members' perspectives. The Journal of Specialists in Group Work, 38(1), 3-29. 
Ryan, G. W., \& Bernard, H. R. (2000). Data management and analysis methods. In N. K. Denzin \& Y. S. Lincoln (Eds.), Handbook of qualitative research ( $\left.2^{\text {nd }} \mathrm{ed}\right)$, pp. 1001-1017. Thousand Oaks, CA: Sage.

Saetren, H. (2005). Facts and myths about research on public policy implementation: Out-of-fashion, allegedly dead, but still very much alive and relevant. The Public Studies Journal, 33(4), 559-582.

Saltzman, L. E., Fanslow, J. L., McMahon, P. M., \& Shelley, G. A. (2002). Intimate partner violence surveillance: uniform definitions and recommended data elements, version 1.0. Atlanta (GA): Centers for Disease Control and Prevention, National Center for Injury Prevention and Control.

Saunders, D. G. (1996). Feminist-cognitive-behavioral and process-psychodynamic treatments for men who batter: Interaction of abuser traits and treatment model. Violence and Victims, 11, 393-414.

Saunders, D. G. (2008). Group interventions for men who batter: A summary of program descriptions and research. Violence and Victims, 23(2), 156-172.

Sarason, I. G., \& Ganzer, V. J. (1973). Modeling and group discussion in the rehabilitation of juvenile delinquents. Journal of Counseling Psychology, 20(5), $442-449$.

Schulz, A. J., Israel, B. A., Zimmerman, M. A., \& Checkoway, B. N. (1994). Empowerment as a multi-level construct: Perceived control at the individual, organizational and community levels. Health Education Research, 10(3), 309327. 
Shepard, M. F., Falk, D. R., \& Elliot, B. A. (2002). Enhancing coordinated community responses to reduce recidivism in cases of domestic violence. Journal of Interpersonal Violence, 17, 551-569.

Skinner, E. A. (1995). Perceived Control, Motivation, and Coping. Thousand Oaks, CA: Sage.

Skinner, E. A. (1996). A guide to constructs of control. Journal of Personality and Social Psychology, 71(3), 549-570.

Smith, C. P. (2000) Content analysis and narrative analysis. In H. T. Reis \& C. M. Judd (Eds.), Handbook of Research Methods in Social and Personality Psychology, pp. 313-338. Cambridge, UK: Cambridge University Press.

Smith, M., \& Doyle, M. (2007). Action research: Traditions and perspectives. Teaching and Learning, 4(2), 24-26.

Smith, J. D., Schneider, B. H., Smith, P. K., \& Ananiadou, K. (2004). The effectiveness of whole-school antibullying programs: A synthesis of evaluation research. School Psychology Review, 33, 547-560.

Snape, D., \& Spencer, L. (2003). The foundations of qualitative research. In J. Ritchie \& J. Lewis (Eds.), Qualitative research practice: A guide for social science students and researchers, pp. 1-23. Los Angeles, CA: Sage.

Spittal, M. J., Siegert, R. J., McClure, J. L., \& Walkey, F. H. (2002). The spheres of control scale: The identification of a clear replicable three-factor structure. Personality and Individual Differences, 32, 121-131. 
Stein, C. H., \& Mankowski, E. S. (2004). Asking, witnessing, interpreting, knowing: Conducting qualitative research in community psychology. American Journal of Community Psychology, 33, 21-35.

Sterling-Turner, H. E., Watson, T. S., \& Moore, J. W. (2002). The effects of direct training and treatment integrity on treatment outcomes in school consultations. School Psychology Quarterly, 17, 47-77.

Stevenson, F. A., Britten, N., Barry, C. A., Bradley, C. P., \& Barber, N. (2002). Perceptions of legitimacy: The influence on medicine taking and prescribing. Health, 6(1), 85-104.

Stryker, R. (1994). Rules, resources, and legitimacy processes. American Journal of Sociology, 99, 847-910.

Swanberg, J. E., Logan, T. K., \& Make, C. (2005). Intimate partner violence, employment, and the workplace. Trauma, Violence \& Abuse, 4, 1-26.

Thibaut, J. W., \& Walker, L. (1975). Procedural Justice: A Psychological Analysis. Hillsdale, NJ: Lawrence Erlbaum.

Thibodeau, R., \& Aronson, E. (1992). Taking a closer look: Reasserting the role of the self-concept in dissonance theory. Personality and Social Psychology Bulletin, 18, $591-602$.

Thompson, R. S., Bonomi, A.E., Anderson, M., Reid, R.J., Dimer, J.A., Carrell, D., \& Rivara, F.P. (2006). Intimate partner violence: Prevalence, types, and chronicity in adult women. American Journal of Preventive Medicine, 30(6), 447-457. 
Tjaden, P., \& Thoennes, N. (1998). Prevalence, incidence, and consequences of violence against women: Findings from the national violence against women survey. National Institute of Justice Centers for Disease Control and Prevention: Research in Brief, 1-16.

Tjaden, P., \& Thoennes, N. (2000). Full report of the prevalence, incidence, and consequences of violence against women. U.S. Department of Justice. Retrieved July 5, 2009, from: http://www.ncjrs.gov/pdffiles1/nij/183781.pdf

Tolman, R. M. (2001). An ecological analysis of batterer intervention program standards. Journal of Aggression, Maltreatment \& Trauma, 5(2), 221-233.

Tremblay, M. A. (1957). The key informant technique: A nonethnographic application. American Anthropologist, 59(4), 688-701.

Tucker, R. K., \& Byers, P. Y. (1987). Factorial validity of Merz's psychological reactance scale. Psychological Reports, 61, 811-815.

Tyler, T. R. (2006). Psychological perspectives on legitimacy and legitimation. Annual Review of Psychology, 57, 375-400.

Tyler, T. R., \& Huo, Y. J. (2002). Trust in the law: encouraging public cooperation with the police and courts. New York, NY: Russell Sage Foundation.

Wallner, J. (2008). Legitimacy and public policy: Seeing beyond effectiveness, efficiency, and performance. The Policy Studies Journal, 36(3), 421-443.

Wang, Q., Bowling, N. A., \& Eschleman, K. J. (2010).A meta-analytic examination of work and general locus of control. Journal of Applied Psychology, 95(4), 761768. 
Wang, S., Tomlinson, E. C., \& Noe, R. A. (2010). The role of mentor trust and protégé locus of control in formal mentoring relationships. Journal of Applied Psychology, 95(2), 358-367.

Ward, C. L., \& Rothaus, P. (1991). The measurement of denial and rationalization in male alcoholics. Journal of Clinical Psychology, 47(3), 465-468.

Weber, M. (1968). Economy and society; an outline of interpretive sociology. New York, NY: Bedminster Press.

Weick, K. E. (1985). Systematic observational methods. In G. Lindzey \& E. Aronson (Eds.), The handbook of social psychology ( ${ }^{\text {rd }}$ ed., pp567-634). Reading, MA: Addison-Wesley.

Wortman, C. B. (1975). Some determinants of perceived control. Journal of Personality and Social Psychology, 31(2), 282-294.

Zelditch, M. (2001). Processes of legitimation: Recent developments and new directions. Social Psychology Quarterly, 64(1), 4-17.

Zelditch, M., \& Walker, H. A. (1984). Normative regulation of power. Advances in Group Processes, 17, 155-178.

Zimmerman, M. A., \& Zahniser, J. H. (1991). Refinements of sphere-specific measures of perceived control: Development of a sociopolitical control scale. Journal of Community Psychology, 19, 189-204. 
Zimmerman, M. A., Ramirez-Valles, J., \& Maton, K. I. (1999). Resilience among urban African American male adolescents: A study of the protective effects of sociopolitical control on their mental health. American Journal of Community Psychology, 27(6), 733-751. 
Appendix A

\section{Oregon State Standards}

\section{BATTERER INTERVENTION PROGRAM RULES}

\section{7-087-0000}

\section{Purpose and Implementation}

(1) ORS 180.700 gives the Attorney General authority, in consultation with an advisory committee, to adopt rules that establish standards for batterers' intervention programs (BIP). OAR 137-087-0000 through 137-087-0100 establish those BIP standards (standards) for intervention services provided to male batterers who engage in battering against women. Additional rules shall be developed later to address standards for intervention services for women batterers and battering in same sex relationships. Nothing in these rules should be construed to prevent a BIP from providing appropriate batterer intervention services to batterers who are not within the scope of these rules at this time.

(2) The purposes of the standards are:

(a) To help ensure the safety of women, their children and other victims of battering;

(b) To help ensure that BIPs use appropriate intervention strategies to foster a batterer's stopping his violence, accepting personal accountability for battering and personal responsibility for the decision to stop, or not to stop, battering; and to promote changes in the batterer's existing attitudes and beliefs that support the batterer's coercive behavior;

(c) To help ensure that BIPs address all forms of battering;

(d) To help ensure that BIPs are culturally informed and provide culturally appropriate services to all participants;

(e) To help ensure egalitarian and respectful behavior by BIP staff toward women and men of all races and cultures;

(f) To help ensure that BIPs provide services that are affordable and accessible for participants, including participants with disabilities;

(g) To provide a uniform standard for evaluating a BIP's performance;

(h) To foster local and statewide communication and interaction between BIPs and victim advocacy programs, and among BIPs; and 
(i) To help ensure that BIPs operate as an integrated part of the wider community response to battering.

(3) Implementation and transition provisions.

(a) A BIP may only apply these standards to BIP applicants who request or are referred for admission to the BIP after the effective date of these rules.

(b) BIPs in operation on the effective date of these rules shall make reasonable efforts to conform their policies and practices with these standards as soon as practicable but no later than six months after the effective date of these rules.

(c) BIPs commencing operations after the effective date of these rules shall comply with these standards as soon as practicable but no later than six months after commencing operations.

Stat. Auth.: ORS $180.070-180.710$

Stats. Implemented: ORS 180.070 - 180.710

Hist.: DOJ 16-2005, f. 11-23-05, cert. ef. 1-1-06.

\section{7-087-0005}

\section{Definitions}

For purposes of OAR 137-087-0000 through 137-087-0100, the following terms have the meanings set forth below.

(1) "Batterer" means:

(a) An adult male 18 years of age or older who engages in "battering" against women; or

(b) A male minor criminally convicted as an adult of conduct against women that constitutes "battering" in whole or in part.

(2) "Battering" includes but is not limited to physical violence, sexual violence, threats, isolation, emotional and psychological intimidation, verbal abuse, stalking, economic abuse, or other controlling behaviors against women in, but not limited to, the following relationships:

(a) A current or former spouse of the batterer;

(b) An unmarried parent of a child fathered by the batterer;

(c) A woman who is cohabiting with or has cohabited with the batterer; 
(d) A woman who has been involved in a sexually intimate relationship with the batterer within the past two years;

(e) A woman who has a dating relationship with the batterer;

(f) An adult woman related by blood, marriage or adoption to the batterer; or

(g) A woman who relies on the batterer for ongoing personal care assistance. "Battering" may or may not violate criminal law and in most instances is patterned behavior.

(3) "Batterer intervention program" (BIP) means a program, whether public or private, profit or non-profit, that is conducted to provide intervention and education services to batterers related to ending their battering.

(4) "Facilitator" means anyone who provides BIP intervention services, whether in a group or class setting, or individually.

(5) "Local Domestic Violence Coordinating Council" (Council) means a council set up by local entities that works to intervene with or prevent domestic violence, and to foster a coordinated community response to reduce domestic violence. A Council shall include representatives of the criminal justice system (such as law enforcement, prosecution, and judiciary) and victims' advocacy programs. A Council may also include medical professionals, mental health professionals, health agencies, substance abuse programs, culturally specific providers, child protective services, child support enforcement, school personnel, senior services, disability services, self-sufficiency services (public assistance) and other applicable programs of the Oregon Department of Human Services (DHS), representatives from faith communities, other community groups, and BIPs.

(6) "Local Supervisory Authority" (LSA) means the local corrections agencies or officials designated in each county by that county's board of county commissioners or county court to operate corrections supervision services, or custodial facilities, or both.

(7) "Mandating Authority" (MA) means the court, district attorney, or corrections system authority that has ordered or required the batterer to participate in a BIP.

(8) "Participant" means a batterer who participates in a BIP.

(9) "Partner" means a female in a past or present intimate relationship with a batterer, including persons described in subsection (2) of this section. A partner may be under the age of 18 and may or may not be an identified victim of the participant's battering.

(10) "Victim" means a female, including a past or present partner, subjected to battering. A victim may be under the age of 18 . In no event shall the batterer be considered a victim for purposes of these rules. 
(11) "Victim advocacy program" (VP) means a nonprofit organization, agency or program that assists domestic violence or sexual assault victims. VPs include, but are not limited to, battered women's shelters, rape crisis centers, and other sexual assault and domestic violence programs assisting victims of battering.

Stat. Auth.: ORS 180.070 - 180.710

Stats. Implemented: ORS 180.070 - 180.710

Hist.: DOJ 16-2005, f. 11-23-05, cert. ef. 1-1-06

\section{7-087-0010}

\section{Integration With Total Community Response to Domestic Violence}

(1) BIP in Wider Community Response. A BIP shall be part of a wider community response to battering and not a "stand alone" form of response. A BIP shall interface with VPs, the Council, the criminal justice system including the LSA, other BIPs, members of the Council, and entities recommended to be part of the Council in OAR 137-0870005(5), to achieve the following objectives:

(a) Increase victim safety and batterer accountability and responsibility;

(b) Increase BIP coordination and communication with the criminal justice system, VPs, other BIPs, and all other entities involved in the total community response to domestic violence;

(c) Decrease the likelihood that a lack of communication between BIPs and other representatives in the community response to domestic violence will jeopardize victim safety or be used by the batterer to manipulate the response system;

(d) Increase the likelihood that BIPs are not working at cross-purposes with other agencies serving domestic violence and sexual assault victims and offenders;

(e) Increase the likelihood that BIPs are providing services representing best practices;

(f) Promote community beliefs and attitudes that discourage battering; and

(g) Support other programs that work to reduce or prevent battering.

(2) BIP and Council. A BIP shall participate in and seek to join the Council if a Council exists in the BIP's service area.

Stat. Auth.: ORS 180.070 - 180.710

Stats. Implemented: ORS 180.070 - 180.710

Hist.: DOJ 16-2005, f. 11-23-05, cert. ef. 1-1-06 


\section{7-087-0015}

\section{Interface Standards -- Victims and Current Partners}

\section{(1) Victim/Current Partner Notification Policies:}

(a) A BIP shall have written policies and procedures that govern BIP contact with identified victims and current partners, and that adequately address the safety of victims, including present and past partners. BIP policies relating to victim or partner contacts shall include a policy as to how to document victim or partner contact information that is consistent with OAR 137-087-0060(4)(b), and shall require the segregation and protection of victim or partner contact records. A BIP shall provide a VP with the opportunity to review and comment on the BIP's proposed victim or partner contact policies and procedures, and any amendments to those policies and procedures, before a BIP adopts them.

(b) In all BIP contacts with victims or partners, the primary goal is the safety of the victim or partner. Any BIP victim or partner contact procedure shall consider victim or partner safety, including the risk of identifying victim location, and the risk of any other unauthorized BIP disclosure of information from the victim or partner.

(c) A BIP shall not pressure, coerce or require victims or partners to disclose any information, have any future contact with the BIP or participant, or attend any BIP or other program sessions, meetings or education groups as a condition of the participant's involvement with the BIP.

(d) Victim or partner contact initiated by a BIP normally shall be limited to the following circumstances:

(A) Notifying the victim or partner that the participant has been accepted or denied admission to the BIP;

(B) Notifying the victim or partner of any conditions imposed on the participant's admission to the BIP;

(C) Notifying the victim or partner of the participant's attendance record;

(D) Notifying the victim or partner that the participant has been suspended, discharged or terminated from the BIP; and

(E) Giving the victim or partner general information about the BIP and community resources, consistent with section (2) of this rule. 
(e) A BIP may adopt a victim or partner contact policy that provides for victim or partner contact using a VP in any of the circumstances described in section (1)(d) of this rule, or other contacts requested by the BIP. This policy may be established by a formal interagency agreement with the VP.

(2) Informational Materials:

(a) A BIP shall prepare for distribution to victims and partners informational materials written in plain language, tailored to the community and responsive to relevant cultural components. The information shall be made available by the BIP upon request to any victim or partner, provided to the VP and LSA, and made available in a form that may be distributed through community resources.

(b) The materials shall include information about the following:

(A) A brief description of the BIP, including program expectations, content and philosophy;

(B) A clear statement that the victim or partner is not expected in any way to help the participant complete any BIP requirements, and that the participant's eligibility for the BIP's services is not contingent in any way on victim or partner participation or on other victim or partner contact with the BIP;

(C) The limitations of BIPs, including a statement that the batterer's participation in a BIP does not ensure the participant will stop any or all battering behaviors;

(D) The high likelihood of participants misusing information they hear in their BIP groups or classes against the victim or partner;

(E) The risk of participants re-offending, or changing their control tactics, or both, while in the BIP or after completion of BIP requirements;

(F) The victim's or partner's right, at her discretion, to contact the BIP, or the facilitators of the group or class the participant is attending, signed up for, or sanctioned into, with any questions or concerns, and the right to have communications kept confidential unless confidentiality is waived by the victim or partner, or unless release of victim information is required by federal or state law or regulation or court order;

(G) A statement that the victim or partner may complain to the BIP, LSA, a VP, or the Council if she has a concern about how the BIP is contacting her;

(H) Contact information related to victim services, such as services offered by VPs in the victim's community, the statewide automated victim notification system (VINE), Oregon crime victims' compensation program, and constitutional and statutory victims' rights; 
(I) Encouragement for victims to make safety plans to protect themselves and their children, including community resources to contact if they believe they are at risk; and

(J) Notification that a VP may be available as a means by which the information set forth in section (1)(d) of this rule may be communicated, thereby allowing the victim to choose to avoid direct contact with the BIP.

(c) Upon request, a BIP shall make a reasonable effort to provide its informational materials in a form suitable for victims or partners with vision impairments or with limited English proficiency.

(3) Imminent Threat to Health or Safety. The BIP shall disclose participant information when, and to the extent, the BIP in good faith believes such disclosure is necessary to prevent or lessen an imminent threat to the health or safety of a person or the public. No authorization to release information is required in such circumstances. The BIP may provide information to a person or persons reasonably able to prevent or lessen the risk of harm, including but not limited to the victim and past or present partners.

(4) Victim-Initiated or Partner-Initiated Contacts. If a victim or partner contacts the BIP, the BIP may provide information and referral as allowed by state and federal confidentiality laws. The BIP shall not inform the batterer about the victim or partner contact. In response to victim-initiated or partner-initiated contacts, any information the BIP wants to request from the victim or partner (e.g., level of concern for her own safety, recent behaviors of her partner) shall only be sought after she has given full consent. The BIP shall make clear that the victim or partner is under no obligation to provide any information, that refusal to do so shall not affect the status of the participant, and that information shared with the BIP may be subject to release if required by federal or state law or regulation or court order. Any information provided to the BIP shall be kept completely confidential unless the victim or partner expressly authorizes its disclosure, or unless release of information is required by federal or state law or regulation or court order. In considering whether to request such information from the victim or partner, the BIP shall prioritize victim or partner safety over any other concerns.

Stat. Auth.: ORS 180.070 - 180.710

Stats. Implemented: ORS 180.070 - 180.710

Hist.: DOJ 16-2005, f. 11-23-05, cert. ef. 1-1-06 
137-087-0020

\section{Confidentiality of Victim and Partner Information}

(1) Confidentiality. All information about or from a victim or partner shall be confidential.

(2) Treatment of Information. Any information the BIP receives about or from a victim or partner is not a part of the participant's record and shall be kept in a secure location separate from information about any participant.

(3) Restriction of Access to Information. A BIP shall restrict access to and use of victim or partner information to only BIP staff who have a specific need to know the information and who are accountable for their access to and use of that information.

(4) Disclosure of Information. Any disclosure of information about the victim or partner shall be made only with the victim's or partner's authorization, or as otherwise required by federal or state law or regulation, or court order.

(5) Notification of Possible Disclosure of Information. If a BIP is put on notice that federal or state law or regulation or court order may require the disclosure of information provided by a victim or partner, the BIP shall immediately notify the victim or partner or the appropriate VP unless such notification would endanger the safety of the victim or partner.

Stat. Auth.: ORS 180.070 - 180.710

Stats. Implemented: ORS 180.070 - 180.710

Hist.: DOJ 16-2005, f. 11-23-05, cert. ef. 1-1-06

137-087-0025

\section{Interface Standards -- Victim Advocacy Programs}

(1) Liaison. A BIP shall designate a program staff member to serve as a liaison to at least one VP and to the Council in the BIP's service area. Through the liaison, the BIP shall:

(a) Work collaboratively with VPs to help ensure that victims are provided informational materials about, or are referred to, a VP or other advocacy, safety planning, or assistance agencies;

(b) Provide BIP policies, procedures and informational materials, and any amendment to such policies, procedures and informational materials, to the VPs and Council for review and comment as to whether the policies, procedures and materials help ensure the safety of victims and follow best practices related to victim notification; 
(c) Work cooperatively with VPs to post, in appropriate locations, information about how victims can contact the BIP, LSA or MA for more information about the BIP;

(d) Work cooperatively with VPs to address VP concerns or problems related to BIP interventions with batterers, or the BIP's relationship with the LSA or MA, or both; and

(e) Develop a procedure to notify VPs when the BIP believes in good faith that such notification is necessary to prevent or lessen an imminent threat to the health or safety of the victim or the public.

(2) Imminent Threat to Health or Safety. A BIP shall disclose participant information to a VP when, and to the extent, the BIP in good faith believes such disclosure is necessary to prevent or lessen an imminent threat to the health or safety of a person or the public. No authorization to release information is required in such circumstances.

Stat. Auth.: ORS $180.070-180.710$

Stats. Implemented: ORS 180.070 - 180.710

Hist.: DOJ 16-2005, f. 11-23-05, cert. ef. 1-1-06

\section{7-087-0030}

\section{Interface Standards -- Criminal Justice System}

(1) Participation in Judicial or Corrections Response. A BIP's intervention services may be part of a judicial or corrections response to battering, either as a condition of probation or post-prison supervision, through a domestic violence deferred sentencing agreement, or as otherwise authorized by law. A BIP is encouraged to use the power of the criminal justice system to hold batterers accountable for their battering.

(2) Liaison. A BIP shall designate a program staff person to serve as a liaison to the LSA and the MA. The liaison shall:

(a) Request information such as court orders, protective orders, no-contact orders, and police reports;

(b) Work collaboratively with the LSA and MA to facilitate coordination of BIP services with supervision requirements so the BIP is not working at cross-purposes with criminal justice system requirements applicable to the batterer;

(c) Report to the appropriate LSA or MA, or both, any known violations of the requirements of a court order, any criminal assaults, or threats of harm to the victim, unless doing so would jeopardize the safety of the victim; 
(d) If violations of BIP program requirements create a significant risk of termination from the BIP, report such violations and risk of termination to the appropriate LSA or MA, or both;

(e) Upon request of the LSA or MA, or both, submit periodic status reports about participant attendance, recommendations for further intervention, and program exit summary; and

(f) Report any other information requested by the LSA or MA to the extent permitted by federal or state law, required by court order, or authorized by the participant.

(3) Communications about Participant Release. In communications about participant release for completion of BIP intervention services, a BIP shall note that such release shall not be interpreted as evidence that the participant is presently non-abusive, as descriptive of his present behavior outside the group, or as predictive of his future behavior.

(4) Consistency with Court Orders. A BIP shall ensure BIP actions are consistent with all court orders, including orders affecting batterer contact with the victim(s) or partner(s).

(5) Training. A BIP shall participate in training and cross-training in conjunction with VPs and criminal justice agencies, and shall offer technical assistance to the criminal justice system and VPs relating to batterers and appropriate intervention strategies to eliminate battering of women and abuse of children.

(6) Imminent Threat to Health or Safety. The BIP shall disclose participant information when, and to the extent, the BIP in good faith believes such disclosure is necessary to prevent or lessen an imminent threat to the health or safety of a person or the public. No authorization to release information is required in such circumstances. The BIP may provide information to a person or persons reasonably able to prevent or lessen the risk of harm, including but not limited to the LSA, the MA, and other law enforcement or corrections personnel.

Stat. Auth.: ORS 180.070 - 180.710

Stats. Implemented: ORS 180.070 - 180.710

Hist.: DOJ 16-2005, f. 11-23-05, cert. ef. 1-1-06 
137-087-0035

\section{Interface Standards -- Other BIPs}

(1) Purpose. The purpose of sections (2)-(4) of this rule is to promote accountability and completion of BIP program requirements and to deter batterers from changing enrollment from one BIP to another BIP to avoid accountability.

(2) Restrictions on Participant Transfer. A participant may not transfer from one BIP to another BIP without the specific authorization of the LSA or MA, or its agent, with supervisory responsibility for the batterer.

(3) Authorization to Obtain Information. After receiving a referral for a new BIP participant from the LSA or MA, a BIP shall require the participant to authorize any former BIP(s) to send the new BIP information about the participant's attendance, participation and payment record, Accountability Plan, exit summary and transfer plan. The new BIP shall promptly request the authorized information from any former BIP(s).

(4) Credit for Sessions. The new BIP may, but is not required to, extend credit for the number of sessions attended at the former BIP; however, the participant shall be required to complete all of the new BIP's program requirements before program completion.

(5) Participation in BIP Organizations. A BIP shall be active in local and statewide BIP organizations to help:

(a) Provide quality services to enhance the safety of victims;

(b) Participate in peer review that fosters statewide compliance with the standards set out in these rules;

(c) Discourage practices by other BIPs that do not comply with these standards;

(d) Assist in the development of relationships with VPs and others in the coordinated community response to domestic violence;

(e) Share research results and new practices with other BIPs; and

(f) Cooperate, to the extent practicable, in research on domestic violence that is approved by the Council and otherwise consistent with victim or partner safety, and collaborate in the production and dissemination of research findings.

Stat. Auth.: ORS 180.070 - 180.710

Stats. Implemented: ORS 180.070 - 180.710

Hist.: DOJ 16-2005, f. 11-23-05, cert. ef. 1-1-06 
137-087-0040

\section{Interface Standards -- Social Service Interfaces}

BIP Responsibilities. To the extent reasonably practicable, a BIP shall:

(1) Establish a liaison with the DHS office in the BIP's service area(s);

(2) Participate in and seek to join the Council if a Council exists in the BIP's service $\operatorname{area}(\mathrm{s})$;

(3) Coordinate with community members to provide community education and public awareness campaigns related to domestic violence;

(4) Assist in training professionals in the community about batterers, services for batterers and accountability for batterers; and

(5) Collaborate with community representatives on issues of public policy related to safety for battered women and children, and intervention with batterers.

Stat. Auth.: ORS 180.070 - 180.710

Stats. Implemented: ORS 180.070 - 180.710

Hist.: DOJ 16-2005, f. 11-23-05, cert. ef. 1-1-06

\section{7-087-0045}

\section{Intervention Strategies}

(1) Appropriate Intervention Strategies. A BIP's intervention strategies shall include, but are not limited to, the following:

(a) Using a culturally specific curriculum whenever possible;

(b) Increasing the participant's understanding of the causes, types and effects of his battering behavior;

(c) Identifying beliefs that support battering;

(d) Using respectful confrontation that encourages participants to challenge and change their beliefs and behaviors;

(e) Addressing tactics used to justify battering such as denial, victim blaming, and minimizing; increasing participant recognition of the criminal aspect of his thoughts and 
behavior; and reinforcing participant identification and acceptance of personal responsibility and accountability for such tactics;

(f) Reinforcing appropriate respectful beliefs and behavioral alternatives;

(g) Promoting participant recognition of and accountability for patterns of controlling and abusive behaviors and their impacts, and participant responsibility for becoming noncontrolling and non-abusive; and

(h) Ensuring that the impact of battering on victims, partners and children, including their safety and their right to be treated respectfully as individuals, remains in the forefront of intervention work.

(2) Inappropriate Intervention Strategies. The following intervention strategies are inappropriate and inconsistent with these standards because each compromises victim safety:

(a) Blaming the participant's decision to batter on the victim's qualities or behaviors;

(b) Coercing, mandating, requiring or encouraging victim or partner disclosure of information or participation in the intervention with the participant;

(c) Offering, supporting, recommending or using couples, marriage or family counseling or mediation as appropriate intervention for battering;

(d) Identifying any of the following as a primary cause of battering or a basis for batterer intervention: poor impulse control, anger, past experience, unconscious motivations, substance use or abuse, low self-esteem, or mental health problems of either participant or victim;

(e) Using ventilation techniques such as punching pillows or encouraging the expression of rage;

(f) Viewing battering as a bi-directional process with responsibility shared by the victim;

(g) Viewing battering as an addiction and the victim as enabling or co-dependent in the battering; or

(h) Using actions or attitudes of moral superiority, or controlling or abusive behaviors toward participants.

Stat. Auth.: ORS $180.070-180.710$

Stats. Implemented: ORS 180.070 - 180.710

Hist.: DOJ 16-2005, f. 11-23-05, cert. ef. 1-1-06 
137-087-0050

\section{Intervention Curriculum}

(1) Basic Intervention Curriculum Requirements. Challenging and confronting participant beliefs and behaviors shall be balanced by creating a safe and respectful environment for change. To accord with these standards, a curriculum for batterers shall include, but is not limited to, the following basic requirements:

(a) Addressing belief systems that legitimize and sustain battering of women and abuse of children;

(b) Informing participants about the types of battering as defined in OAR 137-0870005(2);

(c) Challenging participants to identify the patterns of their battering behaviors and all tactics used to justify battering such as denial, victim blaming, and minimizing; increasing participant recognition of the criminal aspect of his thoughts and behavior; reinforcing participant identification and acceptance of personal responsibility and accountability for all such tactics; and reinforcing alternatives to non-battering behavior;

(d) Encouraging participants to identify the cultural factors that are used by a batterer to legitimize both individual acts of abuse and control and battering as a whole;

(e) Modeling respectful and egalitarian behaviors and attitudes;

(f) Increasing participants' understanding and acceptance of the adverse legal, interpersonal and social consequences of battering;

(g) Increasing the participants' overall understanding of the effects of battering upon their victims, themselves, and their community, and encouraging participants to go beyond the minimum requirements of the law in providing victims and their children with financial support and restitution for the losses caused by their battering;

(h) Identifying the effects on children of battering directed at their mothers, including but not limited to the incompatibility of the participant's battering with the child's well-being, the damage done to children witnessing battering, and educating participants about the child's need for a close mother-child bond, nurturance, age-appropriate interactions, and safety;

(i) Facilitating participants' examination of values and beliefs that are used to justify and excuse battering; 
(j) Requiring participants to speak with respect about their partners and other women, and challenging participants to respect their partner and other women and to recognize their partner and other women as equals who have the right to make their own choices;

(k) Encouraging empathy and awareness of the effect of participants' behavior on others;

(1) Challenging participants to accept personal responsibility and accountability for their actions;

(m) Encouraging participants to challenge and change their own battering beliefs and behaviors; and

(n) Identifying how the participant uses alcohol and other drugs to support battering behaviors.

(2) Accountability Plan. A BIP shall require every participant to develop an Accountability Plan (Plan), and a BIP's curriculum shall provide information that a participant can use to develop his Plan. Accountability planning is an ongoing process intended to increase the batterer's self-awareness, honesty and acceptance of responsibility for battering and its consequences. A participant's Plan shall include specific and concrete steps to be identified and implemented by the participant. A BIP shall always prioritize the safety and best interests of the victim when teaching and reporting on accountability planning. Under no circumstances may the terms of a Plan require, or imply authorization of or permission for, conduct that violates the terms of a court order or other legally binding requirements.

(3) Elements of the Plan. The Plan shall include, but need not be limited to, the following elements.

(a) Description of the conduct to stop and to be accountable for, including:

(A) Description of the specific actions that caused harm, including the entire range of attempts used to control and dominate the victim(s) or partner(s), specific actions that led to the participant being in the BIP, and the participant's intentions or purposes in choosing those actions.

(B) Identification of the beliefs, values, and thinking patterns the participant used:

(i) To prepare himself and plan to batter;

(ii) To justify his battering to himself and to others;

(iii) To blame other persons and circumstances outside his control for his battering; and 
(iv) To minimize and deny his battering, its harmful effects, and his personal accountability and responsibility for the battering and its effects.

(C) Identification of the full range of effects and consequences of the battering on the $\operatorname{victim}(\mathrm{s}), \operatorname{partner}(\mathrm{s})$, children, the community and the participant.

(b) Participant's plan for choosing to treat his former, current or future partner(s) and children in a continually respectful and egalitarian manner, including:

(A) Description of the excuses and underlying beliefs used to justify his battering;

(B) Description of the participant's plan for intervening in his battering to prevent himself from continuing his pattern of battering;

(C) Description of battering the participant is currently addressing and how he is utilizing his Plan;

(D) Description of how the participant is intervening in his battering including the excuses, beliefs and behaviors he is addressing;

(E) Description of how the participant shall choose to act in ways that no longer cause harm to the victim(s), partner(s), children and the community;

(F) Description of how the participant shall take responsibility for choosing to act in ways that no longer cause harm to the victim(s), partner(s), children and the community;

(G) Description of the thoughts, beliefs and actions the participant shall need to change to become non-abusive and non-controlling, and a description of alternative thoughts, beliefs and actions he can use to make non-abusive and non-controlling choices; and

(H) Description of the thoughts, beliefs and actions that the participant uses in other areas of his life that demonstrate that he is already aware and capable of making responsible non-abusive and non-controlling choices.

(c) Acceptance of full responsibility for the participant's choices and their consequences, including:

(A) Acknowledgement that the participant's actions causing harm to the victim(s), partner(s), children and the community were his choice, that he had other options, and that he is fully accountable for his choices and the consequences of those choices for himself and others;

(B) Acceptance of full responsibility for having brought the criminal justice system into his life, and for other consequences of his behaviors; and 
(C) Participant's plan for beginning and continuing to make reparation and restitution for the harms caused, either directly to the victim(s) if appropriate, approved by the $\operatorname{victim}(\mathrm{s})$, and not manipulative, or indirectly by anonymous donation or community service when the victim wants no contact with the participant.

Stat. Auth.: ORS 180.070 - 180.710

Stats. Implemented: ORS 180.070 - 180.710

Hist.: DOJ 16-2005, f. 11-23-05, cert. ef. 1-1-06

\section{7-087-0055}

\section{Culturally Informed Interventions}

(1) Familiarity with Cultural Demographics. A BIP shall maintain familiarity with the cultural demographics of its service area(s) to help the BIP:

(a) Anticipate the various cultural backgrounds that may be represented by participants; and

(b) Identify factors within a particular cultural background that influence battering, or that can be used by the participant to excuse the battering or by the BIP to assist the participant in ending battering without using such factors as excuses for battering.

(2) Scope. For purposes of these rules, cultural groups shall be construed broadly to include race, religion, and national origin, as well as economic and social groups that are identifiable within the BIP's service area(s).

(3) Basic Service Requirement. Culturally-specific services shall be offered to the extent practicable; however, if culturally-specific services are not available, BIPs shall offer culturally informed services.

(4) Culturally Informed Curriculum. A BIP's curriculum shall address, in a culturally informed way, the factors within the particular cultural background of a participant that influence battering. The curriculum shall avoid cultural stereotyping. Facilitators shall show videos and provide information from a variety of cultural perspectives to staff and participants.

(5) Personnel Policies and Procedures. A BIP's personnel policies and procedures shall require training and other activities that:

(a) Promote recognition and understanding of the factors within a particular cultural background that support battering and hinder batterers from stopping violence. Such training shall promote the recognition and avoidance of cultural stereotype views and beliefs by BIP staff. The BIP shall provide staff with the tools to understand their own 
biases and preconceptions about people from specific cultures, and how to avoid such biases or preconceptions in the provision of BIP services and activities;

(b) Inform staff about the negative effects of all forms of oppression and about how individuals within each specific cultural background in the BIP's service area(s) may experience oppression within their own culture or within the dominant community;

(c) Inform staff about how the cultural backgrounds of the populations in the BIP's service area(s) view gender roles and family structure, and how those cultures typically respond to domestic violence, sexual assault, and conflict;

(d) Inform staff about specific strengths of the cultural backgrounds in the BIP's service area(s), e.g., strong kinship ties and work ethic, adaptability of family roles, and egalitarianism, high achievement goals, and strong religious orientation; and

(e) Inform staff about specific traditions within the particular cultural backgrounds in the BIP's service area(s) that support battering and hinder batterers from stopping their battering.

(6) Library of Information and Resources. A BIP shall develop and maintain a library of information and resources about specific cultural backgrounds and culturally sensitive modes of intervention.

(7) Diverse Staff and Environment. To the extent possible, a BIP shall provide a staff and environment that reflect the diversity of cultural backgrounds in the BIP's service area(s).

(8) Relationship with Other Programs. BIPs shall develop relationships with appropriate culturally-specific programs to obtain information or training about the culture, and to refer participants for non-BIP culturally-specific services as needed. BIPs shall cooperate with other BIPs in developing culturally specific programs that comply with these standards.

Stat. Auth.: ORS 180.070 - 180.710

Stats. Implemented: ORS 180.070 - 180.710

Hist.: DOJ 16-2005, f. 11-23-05, cert. ef. 1-1-06 
137-087-0060

\section{Admission Policies and Procedures}

(1) Admission Criteria. A BIP shall have written criteria for accepting or refusing admission requests or referrals. An applicant or referral shall be referred to as a potential participant until the BIP admits the person to the BIP program. The admission criteria shall be available to potential participants, staff, victims, partners and the community, and shall include the following provisions:

(a) A BIP may reject any potential participant the BIP deems to be inappropriate. Inappropriate potential participants may include but are not limited to:

(A) Persons whose conduct causing the referral or application is not battering as defined in OAR 137-087-0005(2); and

(B) Persons whose behavior would be disruptive to meaningful participation in the BIP.

(b) Except for reasons identified in section (1)(a) of this rule, a BIP may not reject a potential participant referred for anger management that is intended to address battering.

(c) After admitting a participant, a BIP may terminate participation on the ground the admission was inappropriate based on the criteria in section (1)(a) of this rule.

(d) If a BIP rejects a referral as inappropriate, or terminates participation of a referral because admission was inappropriate, the BIP shall notify the referral source of the reason for rejection or termination of participation and, when appropriate, may make recommendations for other intervention, treatment services or criminal justice action. The BIP shall notify the referral source within seven working days of the rejection or termination of participation.

(e) A BIP's admission criteria and practices shall not discriminate against any potential participant based on national origin, race, culture, age, disability, religion, educational attainment or sexual orientation. Where there is a substantial barrier to a potential participant's participation in a BIP because of cultural background, language, literacy level, or disability, a BIP shall make reasonable modifications in policies, practices, and procedures to provide BIP services within available resources and in consultation with the referring LSA or MA.

(2) Intake procedures:

(a) A BIP shall use an intake procedure that includes an interview with the potential participant and written documentation of the information collected. 
(b) The BIP shall request information from the potential participant and other relevant sources that the BIP shall use initially to determine whether the potential participant is appropriate and otherwise meets the BIP's admission criteria. That information includes, but is not limited to, the history of battering or violent criminal conduct; history of BIP participation; existence of restraining, protection or no-contact orders; police reports; court orders; involvement with DHS child welfare services; and terms and conditions of probation.

(c) In addition to the information requested under subsection (b) of this rule, a BIP may request additional information from the potential participant and other relevant sources. Any BIP contact to obtain information from a victim or partner shall comply with the victim and partner interface standards in these rules, OAR 137-087-0015. Additional information may be requested by a BIP related to the following:

(A) Factors that may indicate a risk of future violence against the victim or other intimate partner, including but not limited to: safety concerns expressed by the victim; prior assaults against intimate partner(s), children and pets; criminal history; prior violation of conditional release or restraining order(s) or other court orders; history of stalking; extreme isolation or dependence on the victim or partner; attitudes that condone or support domestic violence; history of weapon possession or use; access to firearms; credible threats of injury, death or suicide; lack of personal accountability; minimization or denial of domestic violence history; and association with peers who condone domestic violence.

(B) Factors that may make participation in the BIP difficult or impossible, including but not limited to: lifestyle instability (e.g., unemployment or lack of housing); substance use, abuse or addiction; information about any mental health diagnosis that would affect ability to appropriately participate in the program; negative response to prior services (dropping out, lack of motivation and resistance to change); and persistent disruptive behavior.

(C) Factors that may indicate risk of future violence toward the BIP provider or other participants, including but not limited to a history of weapon use and violent criminal behavior.

(D) Demographic factors that may be used for statistical reasons or programmatic planning, including but not limited to age at time of offense and length of relationship with current or former victim(s).

(3) Participant Orientation to the BIP:

(a) A BIP shall use an orientation procedure to inform the participant about BIP requirements and expectations. A BIP may combine orientation with intake. 
(b) The orientation shall provide the participant with the following BIP materials verbally and in writing:

(A) Statement of the BIP's philosophy consistent with these standards;

(B) Length of program, program attendance policies, and consequences of failure to comply with attendance policies;

(C) Specified fees, methods of payment, and consequences of failure to comply with payment agreements;

(D) Statement of active participation requirement, including personal disclosure and completion of group or class activities and assignments;

(E) Rules for group or class participation and statement of requirement to cooperate with those rules;

(F) Statement of requirement to develop and present an Accountability Plan;

(G) Statement of the BIP's drug and alcohol policy, including but not limited to a prohibition against attending any sessions while under the influence of drugs or alcohol;

(H) Statement of procedure for asserting grievances with the BIP;

(I) Prohibition of weapons possession while on BIP premises or when participating in a BIP function;

(J) Statement of any other BIP rules and conditions for participation in the BIP;

(K) Statement of the BIP's obligation to follow all federal or state laws and regulations, including these standards, relating to required disclosures in the case of: imminent danger to self, victim, current partner or others; or child abuse, elder abuse, abuse of vulnerable adults, or any other circumstances requiring reporting;

(L) Statement of the BIP's confidentiality policy as to participant records, identity of other BIP participants, and information disclosed by other participants in the BIP groups or classes;

(M) Notification that the BIP shall not provide the participant with any information about the victim or partner, either directly or in any judicial or administrative proceeding;

(N) Statement of a requirement that the participant execute all necessary documents to obtain information from, or release of information to, law enforcement, the courts, prior 
intervention or treatment services, social services, victim(s), partner(s), and others as appropriate; and

(O) Statement of criteria for program completion or release.

(4) Participant Record:

(a) A BIP shall keep the following information in each participant's record:

(A) Participant's name, address and phone number;

(B) Name and telephone number of contact in case of emergency;

(C) Fee agreement;

(D) Intake information obtained under section (2) of this rule, name of staff member completing intake, and participant's signed acknowledgement of receiving orientation materials;

(E) Copy of any signed releases of information;

(F) Records of participant's attendance and other participation;

(G) Information received by the BIP after intake, including court orders, police reports, and restraining orders; and information as to any violations, offenses, new arrests or criminal charges during participation;

(H) Except for victim or partner contact information addressed in subsection (b) of this section, documentation of BIP disclosures, including name(s) of person(s) notified due to imminent danger or mandatory reporting consistent with these rules;

(I) Documentation of the participant's status as to completion of the requirements of the program, and any current obstacles to completion;

(J) Exit summary pursuant to OAR 137-087-0070; and

(K) Documentation of any refusal to provide requested information or to sign authorization forms.

(b) The following information is not a participant record and shall not be documented:

(A) Contact or other information about the whereabouts of a victim or partner, other information about a victim or partner not provided by the participant, and any information received by the BIP from a victim or partner; 
(B) Any disclosures to a victim or partner, including any indication that the victim or partner was contacted by the BIP.

(c) Any record of information described in section (4)(b) of this rule shall comply with OAR 137-087-0015.

(5) Participant Access to Records. Subject to denial of access pursuant to subsection (a) of this section, a BIP shall provide the participant an opportunity to review information in the BIP's participant record under section 4(a) of this rule within a reasonable time of receiving a review request, and shall provide a copy of the records upon payment of the cost of duplication.

(a) A BIP may deny or limit a participant's access to the BIP's participant record:

(A) When the BIP determines that disclosure of the records is reasonably likely to endanger the life or safety of the participant or another person;

(B) When the BIP determines that the information was provided to the BIP on the condition that the information not be re-disclosed; or

(C) When the BIP determines that the information was compiled by the BIP in reasonable anticipation of, or for use in, a civil, criminal, or administrative action or proceeding involving the BIP.

(b) If a document in the BIP's records contains any information, obtained from a source other than the participant, about a person other than the participant, the BIP shall redact that information.

(c) Except as expressly provided in these rules, nothing in these rules is intended to create any expectation or right of privacy or confidentiality for any records, files or communications relating to potential participants or participants in BIP services. The BIP may use and disclose information unless and to the extent prohibited or restricted by federal or state law or regulation, including these rules. Use or disclosure of otherwise confidential medical, mental health and treatment records shall comply with applicable federal and state law and regulations.

(d) The BIP shall adopt policies that provide for the confidentiality of a participant record, to the greatest extent practicable consistent with these rules, of a participant who is a defendant participating in a domestic violence deferred sentencing agreement.

Stat. Auth.: ORS 180.070 - 180.710

Stats. Implemented: ORS 180.070 - 180.710

Hist.: DOJ 16-2005, f. 11-23-05, cert. ef. 1-1-06 
137-087-0065

\section{BIP Program Format}

(1) Use of Group or Class Format. A BIP shall ordinarily provide intervention in a group or class format. Exceptions to the group or class format shall be rare and the reasons clearly documented and provided to the Council.

(2) Gender-specific. BIP groups or classes shall be gender-specific.

(3) Group or Class Size. To maximize the impact of the program curriculum, groups or classes shall ideally be composed of 7-12 participants, but shall have no more than 15 participants in addition to the co-facilitators unless approved by the Council and the LSA or MA. Group or class sizes of more than 12 shall be reported to the Council for review and comment.

(4) Co-facilitation. Whenever possible, BIP groups or classes shall be conducted by at least one male and one female to establish an egalitarian model of intervention, increase accountability, and to model healthy egalitarian relationships. The BIP shall notify the Council and LSA when co-facilitation is not occurring, stating the reasons and justifications. At least one of the co-facilitators shall have already met all training requirements as specified in these rules.

(5) Number and Length. After intake, participants shall be involved in the program for at least 48 weekly sessions. Each group or class shall last one and one-half to two hours. There shall be a three month transition period immediately after such completion, with at least one group session each month. A BIP may extend the period of required participation for an individual pursuant to attendance policies and program completion requirements in sections (6) and (7) of this rule.

(6) Written Attendance and Tardiness Policies. A BIP shall adopt written group or class attendance and tardiness policies. At a minimum, such policies shall address punctuality of attendance, criteria for excused and unexcused absences, criteria for a maximum number of absences allowed, and criteria for obtaining exceptions to the attendance policies.

(7) Written Completion Requirements. A BIP shall adopt written program completion requirements, including consequences for excessive absences and other non-compliance, and provide a copy of the completion requirements to the LSA and Council.

Stat. Auth.: ORS 180.070 - 180.710

Stats. Implemented: ORS 180.070 - 180.710

Hist.: DOJ 16-2005, f. 11-23-05, cert. ef. 1-1-06 
137-087-0070

\section{Policies and Procedures as to Termination or Release}

(1) Policies and Procedures. A BIP may release a participant based upon program compliance, or terminate participation based on program non-compliance or for other reasons, as provided in sections (3)-(6) of this rule.

(2) Program Exit Summary. No later than 30 days after the last service contact, a BIP shall prepare for the participant's record an exit summary describing the reason for release or termination and the participant's status. A BIP shall provide a copy of the exit summary to the LSA or MA, or both, or their designees within seven business days after its preparation. In communications about release based on program compliance, a BIP shall note that release is not evidence that the participant is presently non-abusive or nonviolent, does not describe current behavior outside the BIP, and does not predict future behavior.

(3) Release for Program Compliance. A BIP may release a participant based on program compliance only if a participant has achieved:

(a) Compliance with the BIP's attendance policy for the entire time period established in accordance with the BIP's rules;

(b) Compliance with group or class rules throughout intervention services;

(c) Completion of the Accountability Plan; and

(d) Compliance with other BIP rules and conditions for participation in the BIP.

(4) Terminating Participation for Program Non-Compliance. A BIP may terminate participation based on program non-compliance for any of the following reasons:

(a) Failing to maintain regular attendance, consistent with OAR 137-087-065(5) and (6);

(b) Failing to participate during BIP services, or failing to complete assignments, as required by BIP policies provided during orientation pursuant to OAR 137-0870060(3)(b)(D);

(c) Creating an unsafe environment or exhibiting disruptive behavior that undermines the achievement of group or class objectives;

(d) Threatening the safety of the facilitator, staff, or other BIP participants; 
(e) Failing to comply with other requirements of a BIP, including violation of the group or class rules or other conditions that are a part of the BIP's participation requirements;

(f) Failing to comply with the BIP payment agreement; or

(g) Ongoing battering behavior.

(5) LSA Request for Re-admission. Unless the participant was terminated based on section (4)(d) or section (6) of this rule, the BIP may re-admit the participant upon request of the LSA with an increased number of sessions necessary to achieve BIP program completion requirements and other conditions appropriate to the basis for termination.

(6) Terminating Participation for Other Reasons. A BIP may terminate participation because the admission was inappropriate based on the criteria in OAR 137-0870060(1)(a).

(7) Leaves of Absence. A BIP may permit a participant to remain in the BIP while temporarily not attending groups or classes for reasons the BIP determines are justified. Leaves of absence shall be rare and granted only upon proper supporting documentation and when there are no other viable options. The BIP shall immediately inform the LSA or its designee about any leave of absence.

Stat. Auth.: ORS 180.070 - 180.710

Stats. Implemented: ORS 180.070 - 180.710

Hist.: DOJ 16-2005, f. 11-23-05, cert. ef. 1-1-06

\section{7-087-0075}

\section{Post-Release Services}

(1) Service Eligibility. A BIP may provide post-release services to a participant only after his release for program compliance.

(2) Cost of Services. Whenever possible, a BIP shall offer post-release services at little or no cost for former participants to encourage long-term and on-going participation in such services.

(3) Elements of Services. Post-release services may include but are not limited to:

(a) Occasional attendance of the group or class the former participant has left; 
(b) Periodic individual meetings with BIP staff to assess maintenance and to review the Accountability Plan developed pursuant to OAR 137-087-0050;

(c) Periodic group or class meetings of typical or extended length conducted specifically for post-release men; and

(d) Regularly scheduled group or class meetings on an on-going basis.

(4) Limit on Role of Services. Attendance in a post-release group or class shall not substitute for re-enrolling in a BIP or as the primary intervention when there is a new legal charge, court mandate to complete a BIP, or when the participant or partner reports physical violence.

Stat. Auth.: ORS $180.070-180.710$

Stats. Implemented: ORS 180.070 - 180.710

Hist.: DOJ 16-2005, f. 11-23-05, cert. ef. 1-1-06

\section{7-087-0080}

\section{Personnel Standards}

(1) Personnel Policies. A BIP shall adopt the following written personnel policies and procedures applicable to program facilitators, managers or supervisors, administrative staff, volunteers and interns, board members and owners (collectively referred to as "staff" for purposes of this rule except as otherwise specifically identified):

(a) Rules of conduct and standards for ethical practices of staff involved in BIP services with participants or contact with victims or partners;

(b) Standards for use and abuse of alcohol and other drugs, and procedures for managing incidents of use and abuse that, at a minimum, would be sufficient to comply with Drug Free Workplace Standards, 41 U.S.C. 1701 et seq. as described in 45 CFR Part 76 Appendix C;

(c) Compliance with laws relating to domestic violence, sexual assault, stalking and these rules, and applicable federal and state personnel regulations including the Civil Rights Act of 1964 as amended, Equal Pay Act of 1963, the Age Discrimination in Employment Act of 1967, Title 1 of the Americans With Disabilities Act, and Oregon civil rights laws related to employment practices;

(d) Policies and procedures relating to the commission of domestic violence, sexual assault, stalking or abuse by any staff, and providing that the BIP shall terminate employment or volunteer service for such conduct unless the BIP documents reasons for not doing so in the personnel file; and 
(e) Policies and procedures relating to discipline of staff for misuse or unauthorized disclosure of information obtained from or about participants, partners or victims.

(2) Background Checks for Facilitators. A BIP shall use an appropriate method to obtain and review a fingerprint-based state and federal criminal record check for facilitators.

(a) A BIP may ask an applicant, as a condition of employment or volunteer service, to certify whether he or she is, or has been, a respondent in any civil enforcement proceeding, including but not limited to a Family Abuse Prevention Act (FAPA) proceeding involving a restraining or no-contact order, protection order, stalking order, or delinquent child support order. Failure to disclose the existence of a FAPA or no-contact order, protection order, stalking order, or delinquent child support order shall constitute grounds for dismissal or grounds not to rehire.

(b) An applicant shall be disqualified if the individual has ever been convicted of any crime or has been subjected to a FAPA restraining or no-contact order, protection order, or stalking order. The BIP may make an exception to this disqualification if the BIP can document reasons for hiring or retaining the individual consistent with factors in section (5)(d) of this rule. If the facts underlying the conviction were related to domestic violence, the applicant must have completed a BIP with standards similar to these rules, including at least 48 weeks of group classes and implementation of an Accountability Plan, and the applicant must have maintained child support and alimony payments, if any. In addition, a period of more than five years shall have passed since the conviction of the crime or expiration of a court order (e.g., restraining order, no-contact order, protection order, or stalking order), the individual shall have complied with all the terms of his or her sentence or court order, and the individual shall be in compliance with all other qualifications as a facilitator. The BIP shall provide this documentation to the Council for review and comment before hire or continuation of employment, document the response of the Council, and place documentation of the reasons for hiring or retention, and of the Council's response, in the applicant's or employee's personnel file for permanent retention.

(c) A facilitator has an ongoing responsibility to inform the BIP within three working days of any changes in his or her history, including new arrests, convictions, restraining orders or rehabilitation services.

(3) Qualifications of Facilitators. A BIP shall adopt the following minimum qualification standards for facilitators, and as a condition of employment or volunteer services at a BIP, a facilitator shall provide the BIP documentation of compliance with the BIP standards.

(a) Facilitator Experience. A facilitator shall document completion of a minimum of 200 hours of face-to-face contact co-facilitating BIP groups or classes with a facilitator who has met all the facilitator qualification requirements in these rules using a model 
consistent with these rules. A facilitator shall document that this experience was obtained over a period of at least one year. A maximum total amount of 100 hours of this requirement can also be satisfied in one or more of the following ways:

(A) By up to 50 hours of supervised face-to-face contact facilitating victim or survivor support or education classes, or up to 50 hours of working with a caseload primarily of domestic violence offenders on probation or parole;

(B) By up to 50 hours of facilitating offender-related non-domestic violence groups or classes;

(C) By earning a bachelor's degree (50 hours credit for required experience) or master's degree (100 hours credit for required experience) in women's studies, social work, criminal justice, psychology, sociology or other related field from an accredited institution of higher education. The facilitator shall document receipt of the required degree.

(b) Facilitator Training. A facilitator shall document completion of 40 hours of training provided by a nongovernmental (if available) victim advocacy program approved by the Council, and 40 hours of training on batterer intervention that includes the following topics:

(A) Dynamics of domestic violence, including sexual assault and stalking, and power and control models;

(B) Effects on children of exposure to a battering parent and to battering directed at their mothers, including but not limited to, the incompatibility of the battering with the child's well-being, the damage done to children witnessing battering, the child's need for a close mother-child bond, and how abusers use children to gain and maintain control;

(C) Historical views and social attitudes about male dominance, domestic violence including sexual assault and stalking, and the status of women;

(D) Risk factors for future or additional battering, aggressive or controlling behavior;

(E) Cultural competence as it relates to domestic violence, sexual assault, stalking and abuse;

(F) An overview of current state and federal domestic violence laws, including sexual abuse, sexual assault, stalking, child custody and visitation;

(G) An overview of battering behavior and tactics, including sexual abuse and stalking;

(H) Risk of facilitator and system collusion with the BIP participant; 
(I) Appropriate safety guidelines for BIP contact with victims;

(J) An overview of the criminal justice system;

(K) State and local requirements for BIPs, including intervention curriculum requirements in OAR 137-087-0050; and

(L) Importance and elements of a coordinated community response to domestic violence and methods of collaborating with community programs and services.

(c) Culturally Informed Intervention. To satisfy the training requirements in section (3)(b)(E) of this rule, a facilitator shall document completion of seven hours of training in oppression theory, cultural factors and anti-racism as it relates to domestic violence.

(d) Interviewing skills requirement. In addition to the experience and training requirements in sections (3)(a) and (b) of this rule, a facilitator shall document completion of at least 18 hours of training in basic interviewing and group facilitation skills.

(e) Additional training requirement. In addition to the training requirements in section (3)(b) of this rule, a facilitator shall document completion of at least 18 hours of training in substance abuse identification and screening, and at least 12 hours of training in mental health identification and screening.

(f) Documentation requirements. A facilitator shall provide the BIP with documentation of his or her training for each of the topics required by sections(3)(b)-(e) of this rule, and shall include the number of hours and dates of training for each specific topic. If the training in any specific topic was received more than five years before the employment application date or the effective date of these rules, whichever is later, the facilitator must also document completion of additional training in the specific topic(s) during the five years prior to the application date or the effective date of the rules, whichever is later, equal to 25 percent of the required hours in that topic.

(4) Continuing Education for Facilitators. After a facilitator has met the basic qualification standards in section (3) of this rule, the facilitator shall document a minimum of 32 hours over a two calendar-year period of continuing education or training in topics related to the training requirements under sections (3)(b)-(e) of this rule. Not more than eight hours of in-program training, or eight hours of internet or correspondence training, may be used annually to satisfy this biennial requirement.

(5) Background Checks for Staff other than Facilitators. Before employment or volunteer service, a BIP shall use an appropriate method to obtain and review background information for staff and applicants other than facilitators, as follows: 
(a) By having the applicant, as a condition of employment or volunteer service, apply for and receive a criminal history check from a local Oregon State Police office and furnish a copy of it to the BIP; or

(b) By having the applicant, as a condition of employment or volunteer service, sign an authorization for the BIP to contact the local Oregon State Police office for an "Oregon only" criminal history check on the individual.

(c) The BIP may ask the applicant to certify whether he or she is, or has been, a respondent in any civil enforcement proceeding, including but not limited to:

(A) A FAPA proceeding involving a restraining or no-contact order;

(B) A delinquent child support order; and

(C) A protection order or stalking order.

(D) Failure to disclose the existence of a FAPA restraining or no-contact order, protection order, stalking order, or delinquent child support order shall constitute grounds for dismissal or grounds not to hire or to allow volunteer service.

(d) The BIP shall establish policies to evaluate criminal history, if any, in determining whether an applicant shall be hired. The policies shall consider:

(A) The severity and nature of the crime(s);

(B) The number of criminal offenses;

(C) The time elapsed since commission of the crime(s);

(D) The facts of the crime(s);

(E) The applicant's participation in intervention or rehabilitation programs, counseling, therapy, or education evidencing a sustained change in behavior; and

(F) A review of the police or arrest report confirming the applicant's explanation of the crime(s).

(e) If the applicant has been convicted of a crime, the BIP shall determine whether the person poses a risk to the BIP's staff, participants, victims or partners, and whether the criminal history indicates a propensity to collusion with batterers. If the BIP intends to hire the applicant, the BIP shall confirm in writing the reasons for doing so. These reasons shall address the applicant's suitability to work with the BIP's staff or participants 
or to have contact with victims or partners in a safe and trustworthy manner. The BIP shall place this information in the staff's personnel file for permanent retention.

(f) BIP staff have an ongoing responsibility to inform the BIP within three working days of any changes in their history, including new arrests, convictions, restraining orders, nocontact order, protection order, stalking order, or delinquent child support order, or rehabilitation services.

(6) Professional Standards for Staff. A BIP shall include the following professional standards in personnel policies to ensure that staff maintain their professional objectivity and to minimize collusion or any appearance of favoritism or impropriety by the BIP or its staff:

(a) Staff shall not be delinquent in paying any required child support or spousal support;

(b) Staff shall not be involved in any criminal activity;

(c) Staff shall not be under the influence of alcohol or controlled substances while providing BIP services;

(d) Staff shall not use their position to secure special privilege or advantage with participants;

(e) Staff shall not in any way collude with participants. Collusion includes activities such as sympathizing with their complaints against wives; defending their abusive actions for any reason; or laughing at jokes about women, wives, girlfriends or violence. Staff shall not imply that any victim deserves the abuse or show disrespect of any victim.

(f) Staff shall not allow personal interest to impair performance of professional duties;

(g) Staff shall not act as a facilitator for a group or class that includes a family member, personal friend, or past or current business associate of the staff member;

(h) Staff shall not accept any gift or favor from current or former participants, or enter into any business contract or association with participants currently enrolled with the BIP. Cultural or traditional values and customs shall at all times be balanced against this principle;

(i) Staff shall report any potential conflict of interest to BIP supervisors; and

(j) Staff shall immediately report to an appropriate licensing authority, or to the MA or LSA, any unethical or illegal behavior by other staff. A BIP shall not take retaliatory action against a staff person making such report. 
(7) Prohibition of Sexual Harassment or Sexual Exploitation. A BIP shall adopt a written policy prohibiting sexual harassment and sexual exploitation, and shall document in each staff member's file that he or she has reviewed the policy and agreed to comply with it. The policy shall include disciplinary steps available to the BIP if a staff person violates the policy.

(8) Maintenance of Qualification Records. A BIP shall maintain a record documenting each staff member's compliance with applicable qualification standards. The BIP shall maintain the record for three years after the departure of a staff member.

(9) Mentoring and Internships. A BIP is encouraged to provide mentoring or internship opportunities between its staff and staff of other BIPs or VPs to promote professionalism, to provide experienced role models for less experienced staff, interns or volunteers, and to provide cross-training for the BIP's staff. Interns or those being mentored shall be required to comply with all of the supervising BIP's policies and procedures and instruction of the supervising BIP staff.

(10) Facilitators in Training. Individuals in training who have not met all the training and experience requirements applicable to facilitators under these rules may co-facilitate under the active supervision of a facilitator who meets these standards. Facilitatortrainees can co-facilitate under this status for up to two years from the start of the cofacilitating. The facilitator-trainee is immediately responsible for compliance with all other requirements of these rules applicable to a facilitator.

[Publications: Publications referenced are available from the agency.]

Stat. Auth.: ORS $180.070-180.710$

Stats. Implemented: ORS 180.070 - 180.710

Hist.: DOJ 16-2005, f. 11-23-05, cert. ef. 1-1-06

\section{7-087-0085}

\section{Research Programs}

(1) Research. A BIP may use and disclose participant information for research purposes consistent with this rule. Nothing in this section applies to a BIP's disclosure of its own aggregate data or the conduct of its own quality assurance activities. Before making use or disclosure of participant information for research purposes, a BIP shall obtain the following in writing from an independent researcher:

(a) Description of specific actions the researcher shall take to ensure the safety, confidentiality, and autonomy of victims;

(b) An adequate plan to protect participant information from improper use or disclosure; 
(c) Description of steps to ensure that any victim or partner participation, or access to information about a victim or partner by the researcher, shall be based solely on the victim's or partner's informed consent obtained in a manner consistent with section (1)(d) of this rule;

(d) Description of steps to ensure that any procedure involving any victim, partner, or family member, and other collateral contacts including but not limited to past or present employers of the research participant, victim or partner, and a request for participation in the research, shall be developed in consultation with a VP to address victim or partner safety;

(e) Description of steps taken to ensure the input and involvement of community-based domestic violence VPs in the design and implementation of the project;

(f) Description of steps to ensure that the research product shall:

(A) Report both positive and negative data and acknowledge alternative hypotheses, modalities and explanations;

(B) Include a statement about the limitations of self-reporting in accurately measuring a participant's progress or behavior when the research includes information based on selfreporting by participants, including self-reports of program effectiveness; and

(C) Clarify that release for program compliance does not provide any evidence that the participant is presently non-abusive, describe present behavior outside the BIP, or predict future behavior.

(g) Description of a plan to destroy identifiable information at the earliest opportunity or at the conclusion of the research, and to keep confidential any information about, gathered from, or traceable to the victim or partner;

(h) An agreement by the researcher, and his or her agents, not to use or further disclose the research information other than for purposes directly related to the research, and to use appropriate safeguards to prevent misuse of that information;

(i) An agreement by the researcher, and his or her agents, not to publicly identify the research participant or past or current victims or partners; and

(j) An agreement by the researcher to follow federal guidelines relating to Human Subject Research, 45 CFR Part 46, if applicable.

(2) Complaints about Research Conduct. The BIP or other researcher shall make available a person independent of the BIP or other researcher with whom ethical complaints about the conduct of the research can be filed, and establish a procedure for 
such filing. The BIP or other researcher shall inform both the participant and the victim or partner, and any other person or entity upon request, about the complaint procedure.

(3) Reporting Research. The BIP shall require a researcher conducting research on a BIP or BIPs to advise the LSA and the Council about the nature, scope and intent of the research.

Stat. Auth.: ORS 180.070 - 180.710

Stats. Implemented: ORS 180.070 - 180.710

Hist.: DOJ 16-2005, f. 11-23-05, cert. ef. 1-1-06

\section{7-087-0090}

\section{Demonstration Projects}

(1) Demonstration Projects. BIPs shall continue to evolve and change as best practices are developed. These standards are not intended to discourage innovative demonstration projects as long as victim safety and participant accountability are maintained. A BIP may propose to operate a demonstration project by a written request for project approval by the Attorney General's BIP Advisory Committee (Advisory Committee), established under OAR 137-087-0100, that addresses the following:

(a) Identification of the sections and subsections in these rules that project approval would waive;

(b) Relevant research, professional experience, or other credible data showing that the batterer intervention method proposed for the project is an effective and appropriate means of intervention, and that under no circumstances shall the project require actions that shall jeopardize the safety of women, children or the community, collude with the participant, or require victim participation;

(c) Expertise of the BIP to conduct the proposed project and the BIP's ability to maintain such expertise for the project's duration;

(d) A means, independent of the BIP, for evaluating the effectiveness of the project;

(e) The BIP's record, if any, of conducting and completing other programs or projects for private or public entities, including the BIP's record of cooperation in resolving problems identified by such entities;

(f) The geographic location to be served, the participating persons, agencies and organizations, and their respective roles in the project; the length of time for the proposed project, subject to section (3) of this rule; and expected outcomes; 
(g) The involvement, if any, of community-based VPs in the design and implementation of the project;

(h) Position of the LSA, MA and Council in the area to be included in the project as to approval of the project; and

(i) Any additional information the BIP believes is relevant to deciding whether the proposal shall be approved.

(2) Informing Community Partners of the Demonstration Project. After approval of the project by the Advisory Committee and before implementing the project, the BIP shall inform community partners (VPs, LSA, courts, Council, community justice, district attorney's office, alcohol and drug treatment providers and other agencies that come in contact with batterers or with victims or partners) of the demonstration project and changes in the BIP's program design. BIP informational materials shall be revised to state clearly the project's changes so as to avoid any misleading or inaccurate information about the BIP. On a quarterly basis, the BIP shall report to the community partners on the progress of the demonstration project, including concerns about its efficacy. A copy of each report shall also be mailed to the Advisory Committee.

(3) Demonstration Project Time Period. In general, a proposal for a demonstration project shall not exceed an 18 month period. While a demonstration project is being conducted, a BIP may petition to extend the demonstration project. The petition shall provide updated information on all the criteria identified in section (1) of this rule.

(4) Discontinuation of Demonstration Project. After a proposed project is approved, evidence of an increase in batterer abuse, or a decrease in batterer accountability, shall lead to immediate discontinuation of the project. The BIP shall immediately inform the community partners specified in section (2) of this rule, and the Advisory Committee, of the discontinuation of the demonstration project.

Stat. Auth.: ORS 180.070 - 180.710

Stats. Implemented: ORS 180.070 - 180.710

Hist.: DOJ 16-2005, f. 11-23-05, cert. ef. 1-1-06

\section{7-087-0095}

\section{Program Review}

(1) Review of BIP Performance. An LSA, in consultation with the Council, shall periodically review the performance of BIPs located within the jurisdiction of the LSA for compliance with these rules. 
(2) Availability of Records. Except for victim or partner records a BIP shall not disclose, a BIP shall make records available for, and require its staff to cooperate with, program review described in section (1) of this rule.

(3) Distribution of Review. If a review is completed under section (1) of this rule, a copy of the review shall be provided to the BIP executive director, board of directors and owners, and sent by the LSA to the presiding judge and the district attorney for the county in which the LSA operates.

(4) Action on Recommendations. Within 90 days after receipt of the written copy of the review by the BIP, the BIP shall take any corrective actions recommended by the review or advise the LSA in writing why the BIP does not intend to take a particular corrective action. The BIP shall provide a copy of its written response to the Council.

(5) Grievance Policies and Procedures. Each BIP shall develop, implement, and fully inform participants of grievance policies and procedures that provide for receipt of written grievances from participants. The BIP shall document the receipt, investigation, and any action taken as to the written grievance.

(6) Complaint Procedure. Any person, other than a participant, with a concern about a BIP's service delivery may file a written complaint with the BIP. The BIP shall respond to the complaint in writing within a reasonable period of time. In its written response, the BIP shall inform the person that if he or she is not satisfied with the BIP's response, the person may direct his or her complaint to the LSA or the Council.

Stat. Auth.: ORS 180.070 - 180.710

Stats. Implemented: ORS $180.070-180.710$

Hist.: DOJ 16-2005, f. 11-23-05, cert. ef. 1-1-06

\section{7-087-0100}

\section{BIP Advisory Committee}

The Attorney General shall appoint an Advisory Committee composed of representatives from LSAs, BIPs and VPs, and of other members the Attorney General deems appropriate. At the request of the Attorney General and consistent with ORS 180.700, the advisory committee shall evaluate the operation of these standards and provide the Attorney General with any amendments the committee recommends, and shall evaluate requests for demonstration projects that require a waiver of these BIP rules.

Stat. Auth.: ORS 180.070 - 180.710

Stats. Implemented: ORS 180.070 - 180.710

Hist.: DOJ 16-2005, f. 11-23-05, cert. ef. 1-1-06 


\section{Appendix B}

Note: * indicate the item will be utilized to generate compliance scores.

\section{Oregon Batterer Intervention Program Survey}

\section{PLEASE CHECK ONE:}

$\square$ I agree to participate in this survey.

$\square$ I agree to participate in this survey but would prefer to be surveyed over the telephone. If you select this option please provide your telephone number below.

$\square$ I do not agree to participate in this survey.

2. Your name

3. BIP Information

Name of the BIP

Address of the BIP (include any suite or building number if applicable)

BIP Phone Number

BIP Email

In which county is your program located?

In which county (counties) do you provide BIP services? 
4. Your position within the BIP (e.g., director, facilitator)- Check all that apply.
Director/Co-director
$\square$ Facilitator
$\square$ Program coordinator
$\square$ Program manager
$\square$ President/Owner
$\square$ Other (please specify)

5. What year did your BIP begin offering batterer intervention services?

6. How many weekly batterer intervention groups for men are offered by your organization?*

7. On average how many clients do you serve at any given time?*

8. How many culturally specific groups do you offer?

9. If you offer culturally specific groups, please indicate which cultures are offered. Check all that apply.

$\square$ Latino

$\square$ African-American

$\square$ Faith-based

$\square$ Russian

$\square$ Portuguese

$\square$ Native American

$\square$ Italian

$\square$ Other (please specify)

10. How many culturally specific groups do you offer that are in a language other than English? 
11. In what specific language(s)?- Check all that apply.

Spanish

$\square$ Russian

$\square$ Native Language

$\square$ Other (please specify)

12. How does your program address the needs of clients with special needs (e.g., low literacy, deaf, blind, mentally ill, other developmental or physical disability)?- Check all that apply.

$\square$ Hearing impaired services

$\square$ Interpreters available

$\square$ Individual therapy

$\square$ Refer out

$\square$ Evaluation

$\square$ Physical accessibility services

$\square$ Flexible program requirements

$\square$ Other (please specify)

13. What are the weekly fees to attend your program?

Average weekly fees

$\underline{\$}$
$\underline{\$}$
$\underline{S}$

14. Do you offer sliding scale fees?

$\square$ Yes

$\square$ No

15. Are there any circumstances under which a client attends for free?
Yes
$\square$ No

16. If yes, briefly describe these circumstances. 
17. Do other entities (e.g., probation) ever pay (either partially or completely) for clients to attend your program?

$\square$ Yes
$\square$ No

18. If yes, please indicate who else pays for clients to attend you program. - Check all that apply.

$\square$ Criminal justice system

$\square$ Government agencies

Community agencies

$\square$ Child welfare

$\square$ Insurance

$\square$ Family

$\square$ Other (please specify)

19. Does your program have a manual that identifies policies and procedures regarding victims' safety, contact with victims, batterer accountability, transfers from other BIPs, etc.?*

$\square$ No, we don't have these policies and procedures.

$\square$ Yes, we have these policies and procedures but they are not in writing (i.e., they are not in a manual)

Yes, we have these policies and procedures and they are in writing.

20. Are a specific minimum number of weekly sessions required to complete the program?*

Yes

$\square$ No

21. What is the minimum number of weekly sessions (prior to the transition period) required to complete the program?* 
22. Across all clients, what is the average number of weekly sessions participants attend in order to complete the program?

23. Are there specific written criteria for program completion?*
Yes
$\square$ No

24. Which of the following are required for program completion?*
Attendance
$\square$ Participation
$\square$ Complete assignments
$\square$ Complete payments
$\square$ Assessments
$\square$ Comprehension of curriculum
$\square$ Compliance with BIP rules
$\square$ Compliance with group rules
$\square$ Completion of an accountability plan
$\square$ Other

25. Do you keep a record of how many clients complete your program after an intake?*
Yes
$\square$ No

26. What percentage of your clients complete the program? If you don't keep these records, please provide your best estimate (e.g., $25 \%, 50 \%$, etc.)

27. What percentage of your clients are court-mandated to attend? 
28. What percentage of your clients are mandated to attend through DHS/Child Welfare?

29. What percentage of your clients are referred through other channels (e.g., noncourt referrals)?

30. How many batterer intervention program facilitators does your organization employ (not including volunteers or interns)?- If you are a facilitator please include yourself in this number.*

31. How many volunteers or interns fulfill the role of facilitator?

32. Does your program offer co-facilitated groups?
Yes
$\square$ No

33. If yes, how many co-facilitated groups does your program offer?

34. How many of these groups are co-facilitated by facilitators of different genders (e.g., one male facilitator, one female facilitator)?*

35. BIP facilitators must document completion of $\mathbf{4 0}$ hours of victim advocacy training provided by a nongovernmental (if available) victims' advocacy program approved by the Local Domestic Violence Coordinating Council. How many of your facilitators have met this requirement?* 
36. Please describe how your facilitators met this requirement. - Check all that apply.

$\square$ In house training

$\square$ Private nonprofit

$\square$ Shelter/nonprofit

$\square$ Victim advocacy agency

$\square$ Resource center

$\square$ Conferences or workshops

$\square$ Online/Distance learning

$\square$ Education/College

$\square$ Government agencies

$\square$ Other

37. BIP facilitators must also document completion of $\mathbf{4 0}$ hours of training on batterer intervention. How many of your facilitators met this requirement?*

38. Please describe how your facilitators met this requirement. - Check all that apply.

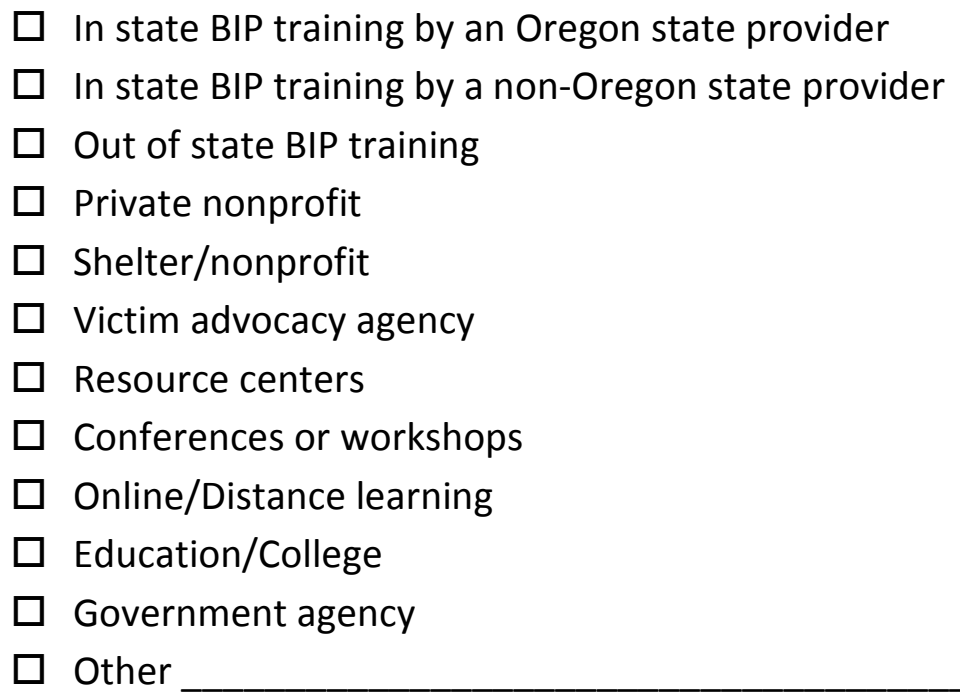


39. Does your county/region have a local domestic violence council?*
$\square$ Yes
$\square$ No
$\square$ Not sure

40. If yes, how often does the council meet?
$\square$ Monthly
$\square$ Every other month
$\square$ Quarterly
$\square$ Less than quarterly
$\square$ Not sure

41. Does a member of your program staff attend meetings held by the council?*
$\square$ Yes
$\square$ No
$\square$ Not sure

42. If yes, how often does the staff member attend these meetings?
Monthly
$\square$ Every other month
$\square$ Quarterly
$\square$ Less than quarterly

$\square$ Not sure

43. Does your program have contact with a nonprofit victims' advocacy program?*
$\square$ Yes
$\square$ No
$\square$ Not sure 
44. If yes, does this nonprofit victims' advocacy program review your program's policies, procedures, and program materials?*

$\square$ Yes

$\square$ No

$\square$ Not Sure

45. Do you have a designated contact within the nonprofit victims' advocacy program that you communicate with?

$\square$ Yes

$\square$ No

$\square$ Not sure

46. Do you have a specific staff member from your program that acts as a liaison with the nonprofit victims' advocacy program?*

$\square$ Yes

$\square$ No

$\square$ Not sure

47. If yes, what is your staff member's name and title?

48. What does this person communicate to the nonprofit victims' advocacy program?

$\square$ How victims can contact the BIP

$\square$ Discussion of problems or concerns

$\square$ How to create plans to address imminent threat

$\square$ Notifications of imminent threat

$\square$ Other 
49. Which components of the criminal justice system does your program communicate with? - Check all that apply.*

$\square$ Local Supervising Authority or LSA (The local corrections agencies or officials designated in each county by that county's board of county commissioners or county court to operate corrections supervision services, or custodial facilities, or both.)

Mandating Authority or MA (The court, district attorney, or corrections system authority that has ordered or required the batterer to participate in a BIP.)

Courts

$\square$ Law enforcement agencies

$\square$ District Attorney's office

$\square$ Domestic Violence Response Team (DVRT)

$\square$ Probation/Parole officer(s)

$\square$ Department of Human Services (DHS) Senior and People with Disabilities DHS Child Welfare

$\square$ DHS Child Welfare

$\square$ Other DHS entities

$\square$ Other public officials

$\square$ Other (please specify)

51. If yes, what does this person communicate to these criminal justice agencies?*

$\square$ Communicate program outcomes

$\square$ Communicate recidivism rates

Communicate dropout rates

$\square$ Communicate regarding attendance

$\square$ Attend regular meetings with probation

$\square$ Contact probation with program concerns

$\square$ Other (please specify) 
50. Does your program have a staff member who is designated to act as a liaison to representatives of the criminal justice system?*

$\square$ Yes

$\square$ No

52. Does your program communicate with or interact with any of the following individuals, groups, or agencies in the community? - Check all that apply.*

$\square$ Church

$\square$ Victim advocacy organizations/Shelters/Resource centers

$\square$ Other BIP or A\&D providers

$\square$ Mental health providers

$\square$ County agencies

$\square$ Family members

$\square$ Government agencies

$\square$ Schools

$\square$ Community organizations

$\square$ Medical resources

$\square$ Employers

$\square$ Defense attorneys

$\square$ Victims

$\square$ Law enforcement

$\square$ Other (please specify)

53. Is the prioritization of victims' safety evident in your program's mission statement?

$\square$ Yes

$\square$ No

We don't have a formal mission statement.

54. Is victims' safety discussed during staff orientation?*

$\square$ Yes

$\square$ No

$\square$ We don't have a formal staff orientation. 
55. Is victims' safety addressed during staff training?

$\square$ Yes
$\square$ No
$\square$ We don't have formal staff training.

56. Is the prioritization of victims' safety evident in the program's curriculum?

$\square$ Yes

$\square$ No

57. Is the prioritization of batterer accountability evident in your program's mission statement?

$\square$ Yes

$\square$ No

$\square$ We don't have a formal mission statement.

58. Is batterer accountability emphasized during staff orientation?

Yes

$\square$ No

$\square$ We don't have a formal staff orientation.

59. Is batterer accountability emphasized during staff training?

$\square$ Yes

$\square$ No

$\square$ We don't have formal staff training.

60. Is batterer accountability part of the curriculum given to clients during group meetings?*

$\square$ Yes

$\square$ No 
61. Does your program require batterers to develop an Accountability Plan?*
Yes
$\square$ Not Sure
$\square$ Other (please specify)

$\square$ No

62. Do you have written policies and procedures regarding client transfers between and to/from other batterer intervention programs?*

Yes, we have these policies and procedures and they are in writing.

$\square$ Yes, we have these policies and procedures but they are not in writing

$\square$ No, we don't have these policies and procedures (Skip to question 64).

63. What considerations are taken when accepting a referral from another program? Check all that apply.*

Attendance

$\square$ Participation

$\square$ Accountability plan

$\square$ Exit summary

$\square$ Transfer plan

$\square$ Payment history

Appropriateness for program

$\square$ Court/PO approval

$\square$ We don't allow transfers

$\square$ Other (please specify)

64. Does your program have written policies and procedures concerning the program's contact with victims and past/current partners?*

$\square$ Yes, we have these policies and procedures and they are in writing.

$\square$ Yes, we have these policies and procedures but they are not in writing

$\square$ No, we don't have these policies and procedures. (Skip to Question 67). 
65. Do these policies define procedures for storing victims'/partners' contact information?*
Yes
No

66. Do these policies define procedures for ensuring victims'/partners' confidentiality?*

$\square$ Yes

$\square$ No

67. Were these policies reviewed by a nonprofit victims' advocacy program?*

$\square$ Yes
$\square$ No

68. Who has access to victims'/partners' records and contact information? - Check all that apply.*

$\square$ Program director(s)

$\square$ Group facilitator(s)

$\square$ Other designated staff member(s)

$\square$ Client(s)

$\square$ Representative(s) from nonprofit victims' advocacy program

$\square$ Other (please specify)

69. Are victims/partners ever contacted by your program or by a contracted victims' advocacy program on your behalf?

$\square$ Yes

$\square \quad$ No (Skip to Question 71). 
70. Under what circumstances are victims/partners contacted? - Check all that apply.*

$\square$ To notify them as to whether the client was accepted or denied admission to the program

To tell them about things the client has said about them during group meetings

$\square$ To tell them about the client's attendance record

$\square$ To tell them that the client has been discharged or terminated from the program

$\square$ To tell them general information about the batterer intervention program

$\square$ To solicit information from them about how a client is doing in the home

$\square$ To inform victims/partners of immediate/imminent threat

$\square$ To provide information about community resources for victims

$\square$ Other (please specify)

71. Does your program offer any services for victims/partners?

Yes

$\square$ No (Skip to Question 76).

72. Please describe the services you offer for victims/partners.

Victim groups/Therapy

$\square$ Couples or marriage counseling

$\square$ Referrals to victim services

$\square$ Parenting classes

$\square$ Aftercare with couples

$\square$ Food and clothing bank

$\square$ Other (please specify)

73. Are these services provided by the same staff that provide services for batterers?
Yes
$\square$ Other (please specify)

$\square$ No 
74. How many staff members who provide services for batterers also provide services for victims/partners?

75. Please describe any additional training requirements (if any) for staff who provide services for both batterers and victims/partners. - Check all that apply.

Licensing or education

$\square$ Victim advocacy training or experience

$\square$ Other (please specify)

76. Does your program distribute informational materials to victims and partners?* Yes

No (Skip to Question 79).

77. What information do these materials cover? - Check all that apply.*

Victims' advocacy resources

$\square$ Community resources

Emergency and/or safety planning resources and suggestions

$\square$ Description of your program

$\square$ Statement(s) about the limitations of BIP outcomes

$\square$ Information about victims' rights

$\square$ Information about contacting or being contacted by the program

$\square$ Other (please specify)

78. Do you offer this information in languages other than English?*

$\square$ Yes

$\square$ No 
79. Does your program offer technical assistance and act as a consultant regarding issues about batterers and batterer intervention programs to: - Check all that apply.

$\square$ Criminal justice agencies

$\square$ Victims' advocacy agencies/programs

$\square$ DHS/Child Welfare

$\square$ Other (please specify)

$\square$ None of the above

80. Does your program assist in the training of others working in the field of intimate partner violence prevention and intervention?*

$\square$ Yes

$\square$ No

81. Does your program belong to any broader (e.g., county, state, or national-level) batterer intervention program organization?*

$\square$ Yes

$\square$ No

82. If yes, please specify which organizations your program belongs to.

83. Please describe the content of the curriculum your program uses. 
84. Please describe any innovative practices your program uses.

85. Please consider the following components of different batterer intervention program curricula. When considering your own program's curriculum and intervention strategies, does it: - Check all that apply.*

$\square$ Use a culturally specific curriculum?

$\square$ Increase clients' understanding of the causes, types, and effects of their battering behavior?

$\square$ View battering as an addiction and the victim as enabling or co-dependent in the battering?

$\square$ Use respectful confrontation that encourages clients to challenge and change their beliefs and behaviors?

Address tactics used to justify battering such as denial, victim blaming, and minimizing?

$\square$ Encourage ventilation techniques such as punching pillows or other expressions of rage?

$\square$ Increase client recognition of the criminal aspect of his thoughts and behavior?

$\square$ Reinforce client identification and acceptance of personal responsibility and accountability for the use of abusive tactics?

$\square$ Blame the client's decision to batter on the victim's qualities or behavior?

$\square$ Reinforce appropriate respectful beliefs and behavioral alternatives?

$\square$ Promote client recognition of and accountability for patterns of controlling and abusive behaviors and their impacts?

$\square$ Use actions or attitudes of moral superiority or controlling or abusive behaviors toward clients?

Ensure that the impact of battering on victims, partners, and children, including their safety and their right to be treated respectfully as individuals, remains in the forefront of intervention work? 
86. Does your program identify any of the following as a primary cause of battering or a basis for batterer intervention? - Check all that apply.*

Poor impulse control

Anger

$\square$ Past experience

$\square$ Unconscious motivations

Substance use or abuse

$\square$ Low self-esteem

$\square$ Client's or victim's mental health problems

$\square$ None of the above

87. Does your program: - Check all that apply.*

Require victim or partner disclosure of information or participation in the intervention with the client?

$\square$ Encourage victim or partner disclosure of information or participation in the intervention with the client?

$\square$ Support, recommend, or utilize couples, marriage, or family counseling or mediation as appropriate intervention for battering?

View battering as a bi-directional process with responsibility shared by the victim?

88. Is your agency for profit or non-profit?

For profit

$\square$ Non-profit

$\square$ Other (please specify)

89. Please indicate how many of your facilitators (not including volunteers and/or interns) hold their highest degree as the degree listed below:

Less than a Bachelor's degree

Bachelor's degree or equivalent

Master's degree or equivalent

Doctorate or equivalent 
90. How many of your program's facilitators are professionally certified and/or licensed within a field related to batterer intervention (e.g., mental health, criminal justice, etc.)?

91. Please indicate how many of your program volunteers and/or interns hold their highest degree as the degree listed below:

Less than a Bachelor's degree

Bachelor's degree or equivalent

Master's degree or equivalent

Doctorate or equivalent

92. How many of your program volunteers and/or interns are professionally certified and/or licensed within a field related to batterer intervention (e.g., mental health, criminal justice, etc.)?

93. How many of your program's facilitators are formerly abusive men or have previously completed a BIP as a client? 
94. Please describe to what extent you feel your program is in compliance with SB 81 and the OARs concerning batterer intervention program guidelines.

$\square$ Not in compliance

$\square$ Some compliance

$\square$ Mostly in compliance

$\square$ Fully in compliance

$\square$ Not sure

95. What (if any) are the biggest barriers to your program's compliance with the BIP guidelines? - Check all that apply.

$\square$ Difficulty hiring qualified facilitators

$\square$ Difficulty retaining qualified facilitators

$\square$ Creating and maintaining necessary collaborations

$\square$ Training requirements

$\square$ Rural location

$\square$ Time and workload difficulties

$\square$ Lack of funding

$\square$ Lack of evidence based requirements and curriculum

$\square$ Inability to accommodate client needs

$\square$ Conflict with county requirements

$\square$ Other (please specify)

$\square$ None of the above

96. Has an outside agency formally reviewed your program for compliance with the state standards in SB 81?

$\square$ Yes

$\square$ No

97. If an outside agency has formally reviewed your program, what was that agency? 
98. In order to ensure that all programs in Oregon have been invited to participate in the survey we would like your help in identifying other programs that provide BIP services. Please list all BIP providers that you are aware of located in your county and any contact information you have for those programs.

99. Thank you for taking the time to complete this survey. Please feel free to use the space provided here to address any other comments, questions, issues, or concerns you may have. 


\section{Appendix C}

\section{Batterer Intervention Program SB 81 Interview Guide \\ Each major bullet point is allotted approximately 5 minutes.}

1. I'd like to begin by discussing when you became aware of standards and what that experience was like. (40 minutes)

- When did you become aware that standards were in place? How did you become aware? Did your initial awareness of the standards have any impact on how you made decisions about your program? (Negative Attitude Maintenance)

○ Probe: Can you tell me more about how they impacted your ability to make decisions?

- Can you describe how you feel about the creation of state standards? What about the people involved in this process? (Perceived Control/Procedural Justice/Legitimacy)

○ Probe: What are your thoughts about creating state policy to influence program practices?

○ Probe: Do you believe that the individuals who participate on the Standards Advisory Committee represent most providers?

Probe: Why do you think this?

- What was your role in creating the Oregon BIP Standards? Did you play a part 
in their development? Are you aware of the process by which the standards were developed? Can you describe the process of creation, as you understand it? (Actual Control)

2. Next, I am going to ask a few questions about your thoughts about the content of the standards?

- What was your initial response when you learned that standards were being implemented/were in place? (Negative Attitude Change and Maintenance)

○ Probe: Providers might have both positive views of the standards, as well as concerns. What was your reaction?

- Is your reaction to the standards similar or different to the response you have had to other policies that affect domestic violence? (Negative Attitude Maintenance)

- If at all, how have your thoughts about the standards changed over time? (Negative Attitude Change)

○ Probe: If your thoughts have changed, what has made them shift?

○ Probe: If not, why do you think you still feel the same way?

- How do you feel about the standards now? (Negative Attitude Change and Maintenance)

- How familiar are you with the content of the standards? (Policy Implementation) 
- Probe: What is your current understanding of what the standards require?

- Probe: Can you describe what the standards entail?

3. Next I would like to talk about the different ways your program has implemented the standards. (45 minutes)

- How has your program changed since the implementation of standards? (Policy Implementation)

- Probe: What practices have shifted due to the standards?

○ Probe: What adjustments have you made because of the standards?

- How has your program stayed the same since the implementation of standards? (Policy Implementation)

- Probe: What practices have remained stable?

- Probe: Do the aspects of your program that have not changed relate to the standards? How so?

- What aspects of the standards have been easiest to implement? (Policy Implementation)

○ Probe: Why?

- What aspects of the standards have been most difficult to implement? (Policy Implementation)

○ Probe: Why?

4. These next questions will touch on the different things that may have make it easier or harder for you to implement the standards. 
- What factors have facilitated you meeting the standards? (Policy Implementation)

○ Probe: Why?

- What factors have been barriers to you meeting the standards? (Policy Implementation)

○ Probe: Why?

- Currently, to what degree do you believe your program practices and policies are in compliance with the standards? How do you gauge your level of compliance? Are you planning to change anything about your program practices that might impact compliance with the standards? (Negative Attitude Maintenance)

○ Probe: Will this make your program more or less compliant?

- Have you received any support in complying with standards? (Policy Implementation)

○ Probe: What types of support have you received?

- What do you think could be done to help programs comply with the standards? (Policy Implementation)

Probe: What resources are needed to do this?

5. Now I would like to discuss your views on the content of the standards, how others understand them, and how they were developed. (35 minutes) 
- Currently, what aspects of the standards seem to be consistent with your understanding of how to best work with abusive men? (Negative Attitude Change and Maintenance/Policy Logic)

- Currently, what aspects of the standards seem to be inconsistent with your understanding of how to best work with abusive men? (Negative Attitude Change and Maintenance/Policy Logic)

- Why do you think this is the case? (Policy Logic)

○ Probe: Do you think they have been created from evidence-based practices?

- Probe: Do you think they have been created from best practices in the field?

Probe: Are your feelings about the standards related to how they were developed?

○ Probe: Is there anything you would change about the standards?

- What is your understanding of the consequences of not complying with the standards? (Absoluteness)

○ Probe: Why?

○ Probe: Has this view changed over time?

○ Probe: What prompted those shifts?

6. Next, I would like to learn more about how others in the BIP and IPV community feel about standards and their importance. 
- How much do you feel compliance with standards is expected or necessary? (Absoluteness)

○ Probe: Who you do you think expects compliance?

- Probe: How have your referral sources changed since the standards came about?

○ Probe: How much do you think they value the standards?

○ Probe: How has this impacted your program?

○ Probe: Is anyone formally monitoring your compliance with standards now or have they in the past? Who?

- Can you describe the extent to which you are involved in the batterer intervention community in Oregon? Do you discuss the standards with other providers? What do you discuss? (Social Norms)

○ Probe: Can you tell me about how those in the field see the standards?

○ Probe: Do you agree with the consensus in the field?

○ Probe: Why or why not?

- To what extent do you feel that you can influence the content or scope of the standards? (Perceived Control)

o Probe: If you had wanted to participate in the creation of standards, do you think you would have been able to do so?

O Probe: If you wanted to see something changed in the standards to what extent do you believe you could influence those changes? 
7. I just have a few more questions today before we wrap up.

- Given all that we have talked about today are there particular things that we discussed that you think are most important to pay attention to? (5 minutes)

- Is there anything you would like to add? Do you have any feedback for me or the interview itself?

- We have discussed many different topics today. When I analyze the interviews I will be pulling out quotes to help explain the experiences that program directors have had implementing standards. The quotes will not be associated with any specific person but I wanted to check in with you to see if there is anything we talked about today that you do not want to be quoted or included? 


\section{Appendix D}

\section{No Longer Functioning Batterer Intervention Program \\ SB 81 Phone Interview Guide}

1. When we attempted to get ahold of you to update the Oregon BIP directory we learned that (name of program) is no longer offering batterer intervention services. Can you tell me why those services are no longer offered? (Impact)

2. Are you aware of the Oregon state standards for BIPs?

o Probe: When did you become aware that standards were in place? How did you become aware? (Negative Attitude Maintenance)

- Probe: What was your role in creating the Oregon BIP Standards? Did you play a part in their development? (Actual control)

○ Probe: Can you describe how you felt about the creation of state standards? What about the people involved in this process? (Perceived control)

- Probe: What was your initial response when you learned that standards were being implemented/were in place? (Negative Attitude Change and Maintenance)

- Probe: Did that initial response change between the time you learned about the standards and the time your program stopped providing services? (Negative Attitude Change and Maintenance)

- Probe: Can you describe the extent to which you are involved in the batterer intervention community in Oregon? Do you discuss the standards with other providers? What do you discuss? (Legitimacy) 
- Probe: Do you think they have been created from evidence-based practices or best practices in the field? (Legitimacy)

3. How much do you feel compliance with standards was/is expected or necessary? (Absoluteness)

4. Do you think the state standards had any impact on your programs functioning or viability? Can you tell me how they impacted your program? (Impact) 


\section{Appendix E}

\section{Interview Codebook}

\begin{tabular}{|c|c|c|c|}
\hline Theme & Code & Definition & $\begin{array}{l}\text { Relevant Interview } \\
\text { Question(s) }\end{array}$ \\
\hline \multirow{6}{*}{$\begin{array}{l}\text { Actual Control } \\
\text { over Standards }\end{array}$} & \multirow{3}{*}{$\begin{array}{l}\text { 1. High actual } \\
\text { control }\end{array}$} & $\begin{array}{l}\text { Served/serves on committee; provided input to committee; } \\
\text { provided input to member of the committee }\end{array}$ & \multirow{6}{*}{$\begin{array}{l}\text { What was your role in } \\
\text { creating the Oregon } \\
\text { BIP standards? Did } \\
\text { you play a part in their } \\
\text { development? Are you } \\
\text { aware of the process } \\
\text { by which the standards } \\
\text { were developed? Can } \\
\text { you describe the } \\
\text { process of creation, as } \\
\text { you understand it? }\end{array}$} \\
\hline & & $\begin{array}{l}\text { Knowledge of how standards were developed and how } \\
\text { committee functions }\end{array}$ & \\
\hline & & $\begin{array}{l}\text { Knowledge of key individuals involved in the standards } \\
\text { creation or refinement process (e.g., members of the } \\
\text { committee) }\end{array}$ & \\
\hline & \multirow{3}{*}{$\begin{array}{l}\text { 2. Low actual } \\
\text { control }\end{array}$} & $\begin{array}{l}\text { Did/does not serve on committee; did not provide input to } \\
\text { committee or member of the committee }\end{array}$ & \\
\hline & & $\begin{array}{l}\text { No/limited knowledge of how standards were developed and } \\
\text { how committee functions }\end{array}$ & \\
\hline & & $\begin{array}{l}\text { No knowledge of key individuals involved in the standards } \\
\text { creation or refinement process }\end{array}$ & \\
\hline
\end{tabular}




\begin{tabular}{|c|c|c|c|}
\hline \multirow{6}{*}{$\begin{array}{l}\text { Perceived } \\
\text { Control over } \\
\text { Standards }\end{array}$} & \multirow{3}{*}{$\begin{array}{l}\text { 3. High perceived } \\
\text { ability }\end{array}$} & $\begin{array}{l}\text { Believes he or she could have participated in the Standards } \\
\text { Advisory Committee if he or she had wanted. }\end{array}$ & \multirow{6}{*}{$\begin{array}{l}\text { To what extent do you } \\
\text { feel that you can } \\
\text { influence the content } \\
\text { or scope of the } \\
\text { standards? If you had } \\
\text { wanted to participate } \\
\text { in the creation of } \\
\text { standards, so you think } \\
\text { you would have been } \\
\text { able to do so? If you } \\
\text { wanted to see } \\
\text { something changed in } \\
\text { the standards to what } \\
\text { extent do you believe } \\
\text { you could influence } \\
\text { those changes? }\end{array}$} \\
\hline & & $\begin{array}{l}\text { Believes that he or she would know who to go to in order to } \\
\text { voice concerns regarding the standards. }\end{array}$ & \\
\hline & & $\begin{array}{l}\text { Believes that he or she would be able to voice concerns and } \\
\text { those concerns would be accounted for by the committee }\end{array}$ & \\
\hline & \multirow{3}{*}{$\begin{array}{l}\text { 4. Low perceived } \\
\text { ability }\end{array}$} & $\begin{array}{l}\text { Believes he or she could not have participated in the } \\
\text { Standards Advisory Committee if he or she had wanted }\end{array}$ & \\
\hline & & $\begin{array}{l}\text { Indicates that he or she is unaware of who to go to in order to } \\
\text { voice concerns regarding the standards }\end{array}$ & \\
\hline & & $\begin{array}{l}\text { Believes that he or she would not be able to voice concerns or } \\
\text { that if voiced, those concerns would not be accounted for by } \\
\text { the committee }\end{array}$ & \\
\hline \multirow{6}{*}{ Initial Response } & \multirow{3}{*}{$\begin{array}{l}\text { 5. Positive initial } \\
\text { response }\end{array}$} & Initial agreement with the overall concept of standards & \multirow{6}{*}{$\begin{array}{l}\text { What was your initial } \\
\text { response when you } \\
\text { learned that standards } \\
\text { were being } \\
\text { implemented/were in } \\
\text { place? }\end{array}$} \\
\hline & & $\begin{array}{l}\text { Initial perception that the standards have not impacted their } \\
\text { ability to make program decisions }\end{array}$ & \\
\hline & & Initial agreement with specific content/scope of the standards & \\
\hline & \multirow{3}{*}{$\begin{array}{l}\text { 6. Negative initial } \\
\text { response }\end{array}$} & Initial disagreement with the overall concept of standards & \\
\hline & & $\begin{array}{l}\text { Initial perception that standards have impacted their ability to } \\
\text { make program decisions }\end{array}$ & \\
\hline & & $\begin{array}{l}\text { Initial disagreement with specific content/scope of the } \\
\text { standards }\end{array}$ & \\
\hline
\end{tabular}




\begin{tabular}{|c|c|c|c|}
\hline \multirow{8}{*}{$\begin{array}{l}\text { Current } \\
\text { Response }\end{array}$} & \multirow{4}{*}{$\begin{array}{l}\text { 7. Positive current } \\
\text { response }\end{array}$} & Current agreement with the overall concept of standards & \multirow{8}{*}{$\begin{array}{l}\text { If at all, how have } \\
\text { your thoughts about } \\
\text { the standards changed } \\
\text { over time? How do } \\
\text { you feel about the } \\
\text { standards now? } \\
\text { Currently, to what } \\
\text { degree do you believe } \\
\text { your program } \\
\text { practices and policies } \\
\text { are in compliance with } \\
\text { the standards? How do } \\
\text { you gauge your level } \\
\text { of compliance? }\end{array}$} \\
\hline & & $\begin{array}{l}\text { Current agreement with the specific content/scope of the } \\
\text { standards }\end{array}$ & \\
\hline & & $\begin{array}{l}\text { Perceived similarities between the content of the standards } \\
\text { and ideal/desired program practices (what program believes } \\
\text { is good) }\end{array}$ & \\
\hline & & $\begin{array}{l}\text { Perceived similarities between the content of the standards } \\
\text { and program practices (what program actually does) }\end{array}$ & \\
\hline & \multirow{4}{*}{$\begin{array}{l}\text { 8. Negative current } \\
\text { response }\end{array}$} & Current disagreement with the overall concept of standards & \\
\hline & & $\begin{array}{l}\text { Current disagreement with the specific content/scope of the } \\
\text { standards }\end{array}$ & \\
\hline & & $\begin{array}{l}\text { Perceived inconsistencies between the content of the } \\
\text { standards and ideal/desired program practices }\end{array}$ & \\
\hline & & $\begin{array}{l}\text { Perceived inconsistencies between the content of the } \\
\text { standards and program practices }\end{array}$ & \\
\hline
\end{tabular}




\begin{tabular}{|c|c|c|c|}
\hline \multirow{7}{*}{ Absoluteness } & \multirow{4}{*}{ 9. Absoluteness } & Beliefs that adhering to the standards is required & \multirow{7}{*}{$\begin{array}{c}\text { What is your } \\
\text { understanding of the } \\
\text { consequences of not } \\
\text { complying with the } \\
\text { standards? How much } \\
\text { do you feel } \\
\text { compliance with } \\
\text { standards is expected } \\
\text { or necessary? Who } \\
\text { you do you think } \\
\text { expects compliance? } \\
\text { How much do you } \\
\text { think they value the } \\
\text { standards? Is anyone } \\
\text { formally monitoring } \\
\text { your compliance with } \\
\text { standards now or have } \\
\text { they in the past? Who? } \\
\text { How have your } \\
\text { referral sources } \\
\text { changed since the } \\
\text { standards came about? }\end{array}$} \\
\hline & & Beliefs that adhering to standards is expected & \\
\hline & & $\begin{array}{l}\text { Experiences with others that require or value compliance } \\
\text { (i.e., probation officers) }\end{array}$ & \\
\hline & & $\begin{array}{l}\text { Experiences that include changes in referral sources as a } \\
\text { result of compliance }\end{array}$ & \\
\hline & \multirow{3}{*}{$\begin{array}{l}10 . \text { Non- } \\
\text { absoluteness }\end{array}$} & Beliefs that adhering to the standards is not required & \\
\hline & & Beliefs that adhering to standards is not expected & \\
\hline & & $\begin{array}{l}\text { Experiences with others that do not require or value } \\
\text { compliance (i.e., probation officers) }\end{array}$ & \\
\hline
\end{tabular}




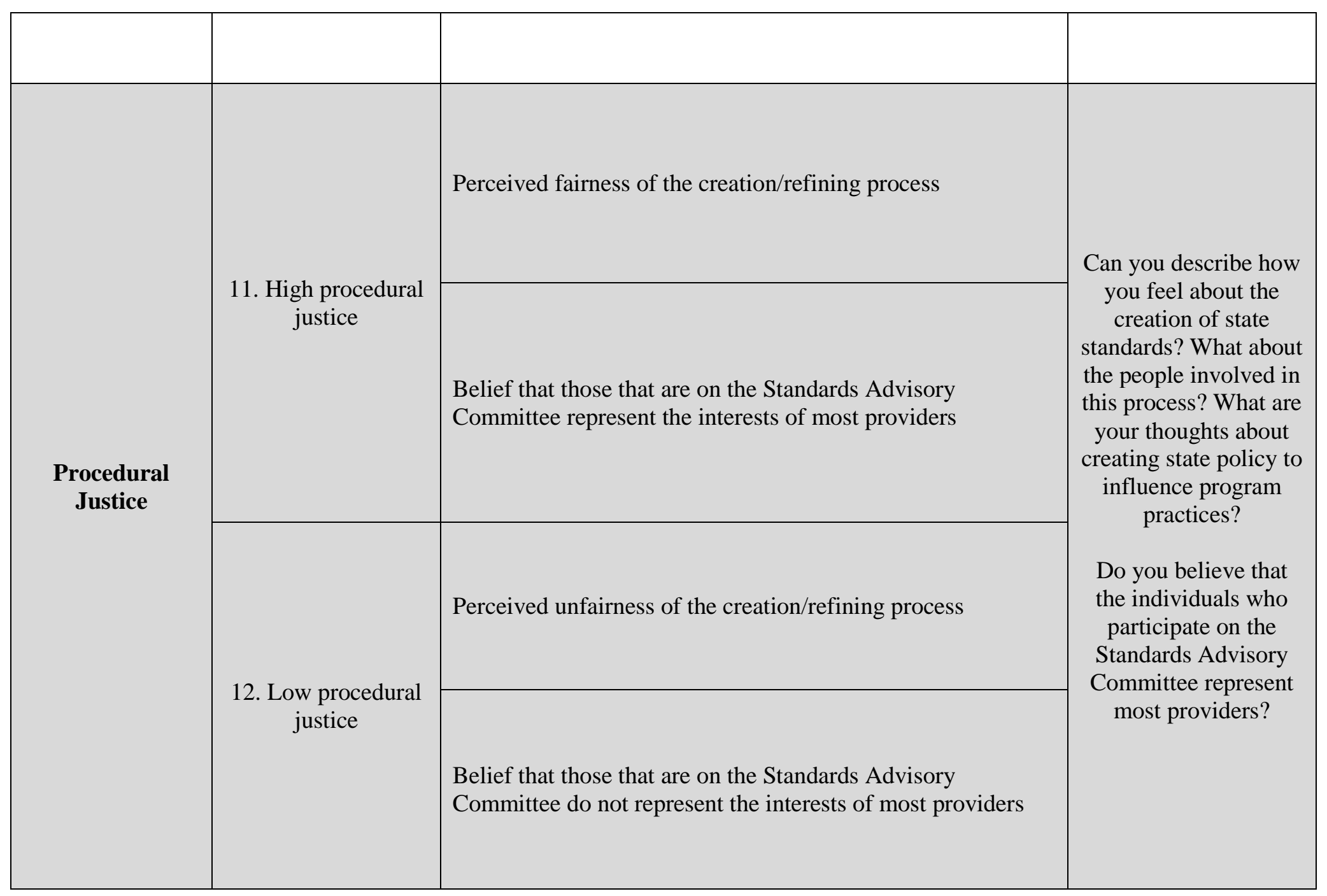




\begin{tabular}{|c|c|c|c|}
\hline \multirow{4}{*}{ Norms } & 13. Positive norms & $\begin{array}{l}\text { Experience of favorable/positive discussion of the standards } \\
\text { among providers / Perception that those in the BIP } \\
\text { community agree with the standards }\end{array}$ & \multirow{4}{*}{$\begin{array}{l}\text { Can you describe the } \\
\text { extent to which you } \\
\text { are involved in the } \\
\text { batterer intervention } \\
\text { community in } \\
\text { Oregon? Do you } \\
\text { discuss the standards } \\
\text { with other providers? } \\
\text { What do you discuss? } \\
\text { Can you tell me about } \\
\text { how those in the field } \\
\text { see the standards? }\end{array}$} \\
\hline & 14. Negative norms & $\begin{array}{l}\text { Experience of unfavorable/negative discussion of the } \\
\text { standards among providers/ Perception that those in the BIP } \\
\text { community disagree with the standards }\end{array}$ & \\
\hline & 15. Limited contact & Did not have much contact or discussion with other providers & \\
\hline & 16. Discussion & $\begin{array}{l}\text { Description of participation in discussion about standards but } \\
\text { no discussion of whether discussion was positive or negative }\end{array}$ & \\
\hline
\end{tabular}




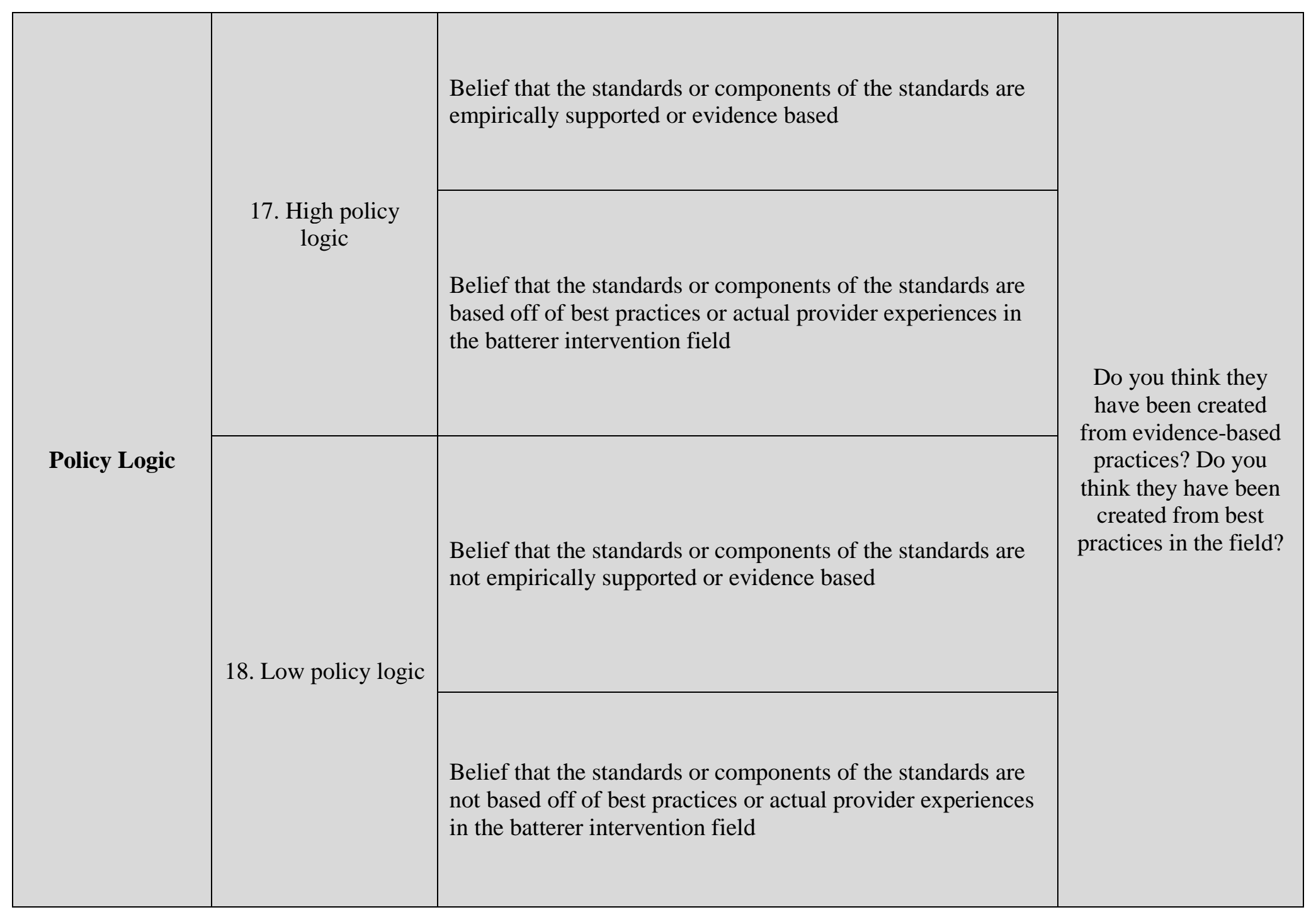




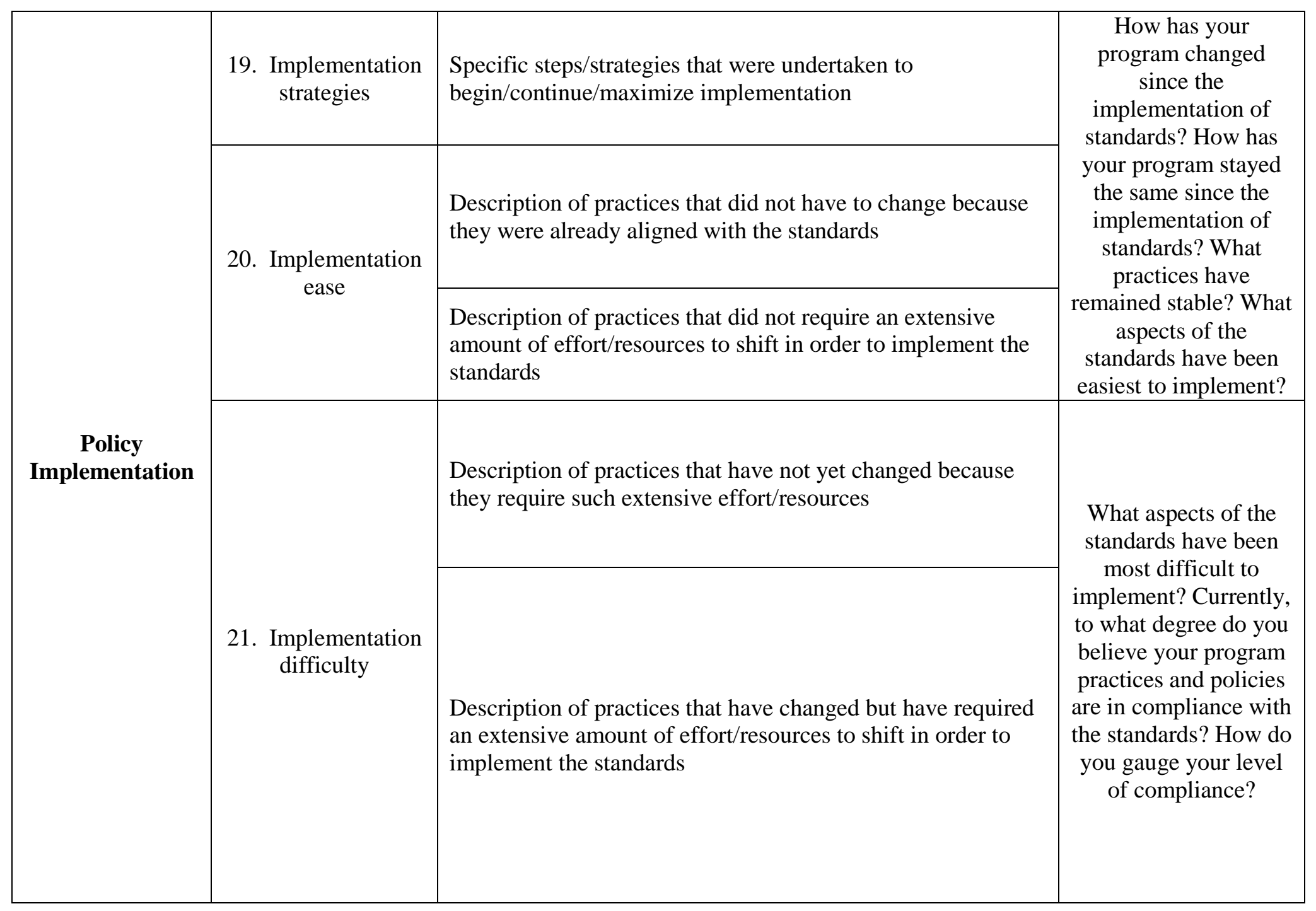




\begin{tabular}{|c|c|c|c|}
\hline \multirow{4}{*}{$\begin{array}{l}\text { Facilitators to } \\
\text { Compliance } \\
\text { Enablers }\end{array}$} & \multirow{4}{*}{$\begin{array}{l}\text { 22. Enablers to } \\
\text { compliance }\end{array}$} & $\begin{array}{l}\text { Description of agencies that enable/encourage } \\
\text { implementation }\end{array}$ & \multirow{4}{*}{$\begin{array}{l}\text { What factors have } \\
\text { facilitated you meeting } \\
\text { the standards? Have } \\
\text { you received any } \\
\text { support in complying } \\
\text { with standards? }\end{array}$} \\
\hline & & $\begin{array}{l}\text { Description of specific activities that enable/encourage } \\
\text { compliance (e.g., trainings, conferences, etc.) }\end{array}$ & \\
\hline & & $\begin{array}{l}\text { Indication that the way in which standards are worded that } \\
\text { enable/encourage compliance }\end{array}$ & \\
\hline & & $\begin{array}{l}\text { Description of program characteristics that enable/encourage } \\
\text { compliance }\end{array}$ & \\
\hline \multirow{8}{*}{$\begin{array}{l}\text { Compliance } \\
\text { Barriers }\end{array}$} & \multirow{4}{*}{$\begin{array}{l}\text { 23. Barriers to } \\
\text { compliance }\end{array}$} & $\begin{array}{l}\text { Description of agencies that are a barrier to implementation } \\
\text { (e.g. community partners) }\end{array}$ & \multirow{4}{*}{$\begin{array}{c}\text { What factors have } \\
\text { been barriers to you } \\
\text { meeting the standards? }\end{array}$} \\
\hline & & $\begin{array}{l}\text { Description of specific activities that make compliance more } \\
\text { difficult }\end{array}$ & \\
\hline & & $\begin{array}{l}\text { Indication that the way in which standards are worded make } \\
\text { compliance more difficult }\end{array}$ & \\
\hline & & $\begin{array}{l}\text { Description of program characteristics that are a barrier to } \\
\text { compliance (e.g., rural location) }\end{array}$ & \\
\hline & \multirow{4}{*}{$\begin{array}{l}\text { 24. Needed support } \\
\text { for compliance }\end{array}$} & Resources needed to increase compliance & \multirow{4}{*}{$\begin{array}{l}\text { What do you think } \\
\text { could be done to help } \\
\text { programs comply with } \\
\text { the standards? }\end{array}$} \\
\hline & & Relationships needed to increase compliance & \\
\hline & & $\begin{array}{l}\text { Support needed to increase compliance (e.g. support from } \\
\text { authority) }\end{array}$ & \\
\hline & & Changes in standards needed to increase compliance & \\
\hline
\end{tabular}




\begin{tabular}{|c|c|c|c|}
\hline \multirow{3}{*}{$\begin{array}{l}\text { Social Action } \\
\text { Research }\end{array}$} & \multirow{3}{*}{$\begin{array}{l}\text { 25. Social Action } \\
\text { Research }\end{array}$} & Survey had impact on practice/gratitude for survey & \multirow{3}{*}{$\begin{array}{l}\text { Is there anything you } \\
\text { would like to add? }\end{array}$} \\
\hline & & Interview had impact on practice/gratitude for interview & \\
\hline & & Specific example of program change due to research project & \\
\hline \multirow{6}{*}{$\begin{array}{l}\text { Impact of } \\
\text { Standards }\end{array}$} & \multirow{3}{*}{ 26. High impact } & $\begin{array}{l}\text { Explanation that program was shut down because standards } \\
\text { were unattainable }\end{array}$ & \multirow{6}{*}{$\begin{array}{l}\text { Can you tell me why } \\
\text { those services are no } \\
\text { longer offered? Do } \\
\text { you think the state } \\
\text { standards had any } \\
\text { impact on your } \\
\text { programs functioning } \\
\text { or viability? Can you } \\
\text { tell me how they } \\
\text { impacted your } \\
\text { program? }\end{array}$} \\
\hline & & $\begin{array}{l}\text { Explanation that program was shut down due to disagreement } \\
\text { or unwillingness to comply with standards }\end{array}$ & \\
\hline & & $\begin{array}{l}\text { Description of experiences in which standards impacted } \\
\text { program referrals or viability }\end{array}$ & \\
\hline & 27. Low impact & $\begin{array}{l}\text { Explanation that standards were unrelated to their program's } \\
\text { closure program }\end{array}$ & \\
\hline & \multirow{2}{*}{ 28. Interest in BIP } & Enjoyed BIP work and/or wishes to continue & \\
\hline & & Did not enjoy BIP work and/or did not wish to continue & \\
\hline
\end{tabular}




\section{Appendix F}

\section{Coding Appendix}

\section{Section 1: Actual Control}

In order to determine experiences of actual control among interview participants, two codes were utilized. Interview transcripts were coded for instances of high actual control and low actual control. The high actual control code $(\kappa=.65)$ was applied when participants reported being involved in the creation or refinement of the standards, experiences as a member of the Standards Advisory Committee, experiences providing direct input to the Standards Advisory Committee or one of its members, or awareness and familiarity with the process by which standards were created and key individuals related to that process. The low actual control code $(\kappa=.87)$ was applied when participants and the program they represent were not involved in the creation or refinement of the standards, were unaware of the process by which standards were refined and created, or did not have knowledge of the individuals who were involved in this process. Across all participants, high actual control was coded 46 times and frequency ranged from $0-12$ instances per participant $(M=3.54, S D=3.41)$. Eleven participants $(85 \%)$ described at least one experience consistent with high actual control. When these eleven participants were considered in isolation, comments representing high actual control were raised an average of $4.18(S D=3.31)$ times per participant. Experiences of low actual control were coded less frequently with 30 instances across all participants and $0-5$ instances per participant $(M=2.31, S D=1.60)$. Ten participants (77\%) described at least one experience of low actual control. When just these 10 
participants were considered, comments relating to low actual control were described an average of $3(S D=1.05)$ times per participant.

While three programs exclusively reported experiences consistent with high actual control and two participants exclusively reported experiences consistent with low actual control, most participants $(n=8,62 \%)$ voiced experiences of both high actual control and low actual control. Consequently, a ratio was created to determine the proportion of high actual control experiences compared to all experiences relating to control for each participant. Specifically, for each participant, the frequency of the high actual control code was divided by the frequency of the high actual control code and frequency of the low actual control code combined. This provided a ratio indicating the proportion of high actual control experiences compared to all experiences related to control combined. These ratios ranged from 0 to 1.00 , with zero indicating no experiences of high actual control and one indicating only experiences of high actual control. The average actual control ratio was $.46(S D=.52$, ranging from $0.00-1.00)$, indicating that across all programs, $46 \%$ of experiences described by participants related to actual control were indicative of high actual control.

This evaluation of the interview data highlights several features of the experience of actual control. First, most participants described experiences consistent with both high actual control and low actual control. This indicates that experiences of control, as operationalized, were not bound to just one type of control experience. Instead, it was possible for participants to describe some experiences indicative of high actual control, while still experiencing other situations consistent with low actual control. Second, while 
most participants experienced both aspects of control, the majority of participants experienced fewer experiences of high actual control than experiences of low actual control over the standards. Thus, given the operationalization of these codes, it appears that more participants were uninvolved with the standards process, unknowledgeable about those involved in the creation of the standards, and unaware of the process by which standards were created than were involved or knowledgeable about the key individuals related to the standards and the creation process. While an understanding of the number of high actual control and low actual control responses is valuable in order to determine and compare the frequency of high actual control and low actual control experiences, the specific responses obtained through the interview process provided ample descriptions of the quality of these experiences. Thus, the content of experiences related to control were assessed to better understand the nuances of both high actual control and low actual control in this sample.

The content of the interview responses revealed several greater depth into experiences of actual control. First, the content of descriptions consistent with high actual control was examined. The specific substance of these experiences varied across participants, though each was indicative of an experience of control over the creation and/or maintenance of the standards (i.e., served on Standards Advisory Committee; provided input to the Standards Advisory Committee; aware of creation process; aware of key individuals). Three participants reported being part of the Standards Advisory Committee at some point during the development or refinement of standards. For instance, one participant described how their participation and attendance at meetings 
leading up to the development of the Standards Advisory Committee played a crucial role in being asked to formally join, "I think somebody nominated me because I kept attending and being involved... and then... I was voted on as a member". This participant highlights the notion that previous activity and involvement in the BIP community contributed to their active role moving forward in the standards development process. Another participant who was part of the Standards Advisory Committee described the vision for representation of different stakeholders in the community collaborative response on the Standards Advisory Committee. Specifically, they remember that Hardy Myers, the Attorney General at the time, “...wanted representatives from various fields to be part of the [standards creation] process. Victim's services, batterer intervention providers, community partners... They pulled community partners and wanted various participants to kind of steer that committee...". This indicates that there appears to have been some effort to invite diverse stakeholders related to IPV to be part of the Standards Advisory Committee. This process led to that particular program director joining the Standards Advisory Committee. As the participant explained, "I thought it better to be at the table and a part of the discussion than to not be." While some experiences of high actual control were the result of participation on the Standards Advisory Committee, one participant described providing feedback directly to the Standards Advisory Committee or one of its members. Specifically, this participant worked with a larger group of providers to generate and disseminate feedback to the Standards Advisory Committee. This participant viewed this endeavor as both successful and safe, “...it was nice to [give feedback] as a group because we could be anonymous and not have to feel that we're [at 
risk]... they made some changes because of that and I appreciate that". While this participant did not serve on the Standards Advisory Committee, he or she was able to voice their thoughts and opinions about the content of the standards. Further, due to the group structure of the feedback, they were able to provide suggestions without fear of negative perceptions or consequences that might have occurred if one program in isolation suggested changes to the standards.

Finally, participants demonstrated high actual control through awareness of the process or key individuals related to the creation and maintenance of standards. Other than those serving on the Standards Advisory Committee, three participants indicated they were at least somewhat knowledgeable about how the standards were created and maintained. One of these participants was able to describe a great deal of detail regarding the development of standards. The remaining two participants were aware of key aspects of the process but were not able to provide an immense amount of detail about those components. For instance, when asked to describe their knowledge of the standards creation process, one participant was able to accurately outline the process in great detail. This participant remembered:

There was a series of meetings, I'm aware of that. They had a number of providers and corrections officers and attorneys and victim advocates come down to Salem and... have a discussion about things that were already happening, things that needed to change, things that were going to be new.

This participant was perhaps more aware than others because at one point they were invited to join the Standards Advisory Committee, though they had to decline the offer due to other responsibilities. Others only had a vague sense of the process, though they were aware of critical components of the creation process. For instance, one participant 
reported awareness of the Standards Advisory Committee and accurately believed that BIP providers were represented, "I know it was a committee of people. I think there was a committee of people who ran programs that sat on a board or whatever it was that set up the standards". Thus, these participants were at least somewhat aware of the creation process, even if they could not outline specific details of that process. In addition to understanding the process, many participants could name key individuals involved in the state standards. Specifically, other than those already serving on the Standards Advisory Committee, eight participants were able to name at least one individual who serves on the Standards Advisory Committee. This indicates that the majority of providers have at least heard and retained the names of the key individuals responsible for the standards.

In addition to experiencing high actual control, participants also described experiences consistent with low actual control. Experiences of low actual control took three forms: the indication that the participant did not participate in the Standards Advisory Committee; is unaware of the process by which standards were created; or is unaware of key individuals related to the standards. When asked about involvement with the Standards Advisory Committee during the creation of the standards all 10 of the individuals who reported at least one experience consistent with low actual control indicated that they do not currently and have not ever served on the Standards Advisory Committee. Reasons for inactivity were almost exclusively tied to a lack of information about the Standards Advisory Committee and standards development process. Only two participants described a reason for inactivity other than lack of knowledge. One participant indicated that he or she was just starting their program when the standards 
development was underway and because of this they did not feel competent enough to participate in the creation of standards. Specifically, this participant remembered, "I think that in the beginning I didn't have a lot of input because I was so new at the time when they were first being developed". The second participant was asked to join the Standards Advisory Committee but declined due to time constraints:

At one point I was invited to be a part [of the Standards Advisory Committee] but at that time, beginning an agency and being in between finding a curriculum that was going to fit, I didn't think I had enough time to devote to it. In hindsight I wish I would have.

Thus, this individual did have the opportunity to influence the standards but had to prioritize other obligations and therefore declined the opportunity. This participant was clear throughout their interview that they regretted this decision and wish they had played a more active role in the standards creation.

Beyond these two participants who had specific rationale for deciding not to become involved in the standards creation process, the remaining participants indicated inactivity and lack of awareness about the standards creation process. Specifically, when asked about whether these participants played a role in the standards process these participants tended to answer with a simple, "No". When asked to describe their understanding of the process by which the standards were created, it became clear that these participants had very little information about how the standards were developed. For example, one participant explained, "I don't know what the process was at all." An additional participant reiterated this point, “I know nothing. I don’t know when, I don’t know how, I don't know why". This lack of knowledge as to how the standards were created clearly raised questions for participants as to what voices contributed to their 
development. One provider indicated that because they do not know how the standards were put together, they are unsure about who was actually represented when they were developed, “I don't even know who was represented there or if anybody there was domestic violence trained. That would have been nice to know". Similarly, another participant voiced their concern about the lack of provider representation, "I don't know if batterer intervention had a representative there, I'm not positive". Further, when asked if the participant knew of any individuals that contributed to the standards, two participants indicated they did not know of anyone related to the standards. One participant explained that their lack of knowledge about the standards creation process is the result of their unawareness regarding who was responsible for the development of standards, "I don't know exactly where they came from because I don't know who developed them". Together these experiences indicate that most providers did not play an active role in the creation of the standards by participating on the Standards Advisory Committee. Further, the majority of these participants did not decide whether or not they were interested in participating on the Standards Advisory Committee but instead the lack of activity appears to stem from a lack of awareness. Thus, while programs that participants represent were functioning while the standards were developed and introduced, most did not play an active role in their development.

\section{Section 2: Perceived Control}

In order to examine perceived control among interview participants, interview transcripts were coded for instances of high perceived ability; high procedural justice; low perceived ability; and low procedural justice. These two sets of codes (i.e., high and 
low perceived ability and high and low procedural justice) were applied separately, rather than combined into one high and one low perceived control code in order to permit the further examination of the procedural justice codes in a subsequent research question. While the codes are ultimately combined to create a gauge of perceived control, each code was also examined independently in order to provide insight into how these components of perceived control were experienced by participants. The high perceived ability code $(\kappa=.63)$ was applied when participants reported believing that they could have participated in the standards creation process if they had wanted to, believing they know with whom they can raise concerns about the standards, or believing that their concerns would be taken seriously. The low perceived ability code $(\kappa=.75)$ was applied when participants reported not believing they could have participated in the standards creation process if they had wanted to, believing they do not know with whom to raise concerns about the standards, or not believing that their concerns would be taken seriously. The code of high procedural justice $(\kappa=.66)$ was applied when participants indicated they viewed the standards process as fair or they believed the Standards Advisory Committee represented most providers. The code of low procedural justice $(\mathbf{\kappa}=$ .55) was applied when participants perceived the standards process as unfair or the committee as unrepresentative of most providers.

Across all participants, high perceived ability was coded 18 times and frequency ranged from $0-4$ instances per participant $(M=1.38, S D=1.45)$. Eight participants described at least one belief consistent with high perceived ability. When these eight participants $(62 \%)$ were considered in isolation, perceptions consistent with high 
perceived ability were discussed an average of $2.25(S D=1.17)$ times per participant. Experiences of low perceived ability were coded more frequently with 21 instances across all participants and $0-6$ instances per participant $(M=1.62, S D=1.66)$. Ten participants (77\%) described at least one experience consistent with low perceived ability. When just these 10 participants are considered, perceptions representative of low perceived ability were coded $2.10(S D=1.60)$ times per participant. The high procedural justice code was used 12 times with frequency ranging from $0-5$ instances per participant $(M=.92, S D=1.55)$. This code was utilized fairly infrequently with only five participants (38\%) describing perceptions consistent with high procedural justice at least once. Across these five participants, comments were coded as high procedural justice an average of 2.40 times $(S D=1.67)$ per interview. The low procedural justice code was used 32 times, with frequency ranging from $0-7$ instances per participant $(M=2.46, S D$ $=2.54)$. Nine participants (69\%) described perceptions consistent with low procedural justice at least once. Across these nine participants, comments were coded as low procedural justice an average of 3.56 times $(S D=2.30)$ per interview.

The construct of perceived control was operationalized to include experiences consistent with the perceived ability codes and the procedural justice codes. Thus, to gain a complete picture of perceived control, the high perceived ability and high procedural justice codes were combined and the low perceived ability and low procedural justice codes were combined. This provided a composite of the number of experiences consistent with high and low perceived control. Combining material coded as high perceived ability and high procedural justice produced a gauge of high perceived control, while combining 
material coded as low perceived ability and low procedural justice produced a gauge of low perceived control. High and low perceived control perceptions were then examined. Across interviews there were 30 instances of high perceived control, with frequency ranging from $0-6$ instances per participant $(M=2.31, S D=2.25)$. Nine participants (69\%) described perceptions of high perceived control at least once. Across these nine participants, comments indicative of high perceived control were detected an average of 3.33 times $(S D=1.93)$ per interview. Low perceived control was evident 53 times across all participants, with frequency ranging from $0-8$ instances per participant $(M=4.08$, $S D=2.60)$. Twelve participants $(92 \%)$ described perceptions consistent low perceived control at least once. Across these 12 participants, comments indicative of high perceived control were detected an average of 4.42 times $(S D=2.39)$ per interview. The average perceived control ratio was $.34(S D=.29$; ranging from $0.00-1.00)$, indicating that across all programs, $34 \%$ of experiences described by participants related to perceived control were indicative of high perceived control.

Most participants $(n=8)$ voiced experiences of both high perceived control and low perceived control. Only one participant exclusively reported experiences consistent with high perceived control, while four participants reported experiences exclusively consistent with low perceived control. Given this, a ratio was created to determine the proportion of high perceived control experiences compared to all experiences relating to control for each participant. Specifically, for each participant, the frequency of the high perceived control perceptions was divided by the frequency of the high perceived control perceptions and frequency of the low perceived control perceptions combined. This 
provided a ratio indicating the proportion of high perceived control perceptions compared to all perceptions related to perceived control combined. These ratios ranged from 0 to 1.00 , with zero indicating no perceptions consistent with high perceived control and 1.00 indicating only perceptions consistent with high perceived control.

This evaluation of the interview data highlights several features of the experience of perceived control over the creation and refinement of the standards. First, as was seen when examining actual control, participants described experiences consistent with both high and low perceived control, indicating that perceptions of both can exist. Second, while most participants experienced both aspects of control, the majority of participants experienced fewer experiences of high perceived control than experiences of low perceived control over the standards. Thus, it appears that more participants tend to believe they do not have the ability to participate or would not be taken seriously, and do not believe the standards process and committee members represent most providers than believe they could participate, their voices would be heard, the standards process was fair, and the committee members represent most providers. Next, the content of perceptions related to perceived control were assessed to better understand the nuances of the four codes utilized for this research question. Specifically, the content of responses coded as high perceived ability, high procedural justice, low perceived ability and low procedural justice was examined.

The high perceived ability code was applied to include several types of perceptions in the current study, including beliefs that participation in the creation or refinement of the standards was/is possible and feedback regarding the standards would 
be taken seriously. It is important to note that three of the eight participants who described perceptions of high perceived ability are individuals who served or currently serve on the Standards Advisory Committee. These individuals are in a slightly different position in terms of perceptions about the possibility of future participation, as they are already integrated into the functioning of the Standards Advisory Committee. All three of these participants indicated they were confident that future participation would be easily achieved. For example, one participant reported that future participation would not be problematic due to their prior involvement, "Well, yeah [it would be possible]. Only because we have [our program director] right on the committee. So, to some extent yes [we could participate], a major extent". Of the remaining five participants, when asked if they believe they could potentially participate on the Standards Advisory Committee if they desired, four participants endorsed this notion. For instance, one participant reported the belief that the Standards Advisory Committee is an open group and participation would be possible, "I don't think it's a closed group and certainly yes, I would be amenable to joining". Additionally, of these five participants, all five believe they know who they would go to or know how to start finding information about where to go with concerns about the standards. One participant described the process they would pursue to raise any concerns:

I would try and make sure [a specific member of the committee] knew who I was and that [they] needed to get me meeting information and I would go and make sure I had plenty of documentation of my ideas and justification for it and make them have a discussion about it.

Thus, this individual has a plan for whom they would contact and how they would present their ideas in a way that they perceive would allow participation in future 
decisions regarding the standards. Another participant indicated that their route to connecting to the right people would include getting in touch with the researcher, "I'd probably call you first. I'd be like, who would I talk to?". While not as detailed as the action steps described by the other participant, this individual still believes they would contact someone, in this case the researcher, to connect them and facilitate participation. A belief that concerns would be validated was also common across the eight participants who discussed at least one aspect of high perceived ability, with seven participants indicating their ideas would be validated. One participant indicated that if they were to draw attention to a matter, they believe it would be addressed, "I do think that for me personally, if I made a big enough stink about it, at least people would hear, they would be forced to hear". This participant has served or currently serves on the Standards Advisory Committee, so it is not surprising that they believe their voice would be heard. Another participant who was not part of the Standards Advisory Committee felt confident that if they were to join the committee now, their perspective would be valued:

I think that if I were to be involved in the re-creation of this, my input would be considered and there would be some adjustments made as a result of my input. So I do think that my voice would be heard.

These comments highlight that the majority of participants do believe that could potentially have some ability to participate and their concerns would be taken seriously.

High procedural justice was coded to capture participants' perceptions of fairness. Five participants reported at least one experience or perception indicating that they believed the Standards Advisory Committee attempted to be fair in the standards 
process and had representation on the committee. One participant explained that they believed the spirit behind the creation of the standards was positive and fair:

I think the fact that in good faith, people got together and tried to glean information from lots of resources, from lots of disciplines, gives credence to the fact that this group of people worked for two years to develop these standards and they weren't doing it ... for a self-servicing purpose but for the betterment of all the programs.

This comment points to the participant's belief that the standards were created from input from diverse stakeholders and were developed in the spirit of improvement. Another provider shared their gratitude for the ways in which the Committee has responded to and incorporated feedback, "I think for the most part I’ve been really appreciative... I think [the committee] has done a really good job of hearing us". Thus, this participant has seen fairness in the process of developing standards by observing their views and input taken seriously. A third participant indicated that the ultimate outcome of the process was a positive one, "Certainly I think that good was done by the people that were there, to come up with these standards and put this into policy."

In addition to these comments related to fairness of the standards creation process, three participants stated that they believed the committee represented most providers. As one participant described, "I thought it had the concerned players, you know, the stakeholders, if you will". Another reinforced this, "I don't think that it was exclusionary, I think there were a certain group of individuals that just made it a priority to be part of this when it was starting up". The content of material coded as high procedural justice indicates that a minority of participants believe the standards creation process had elements of fairness and representation was adequate. While some providers reported 
experiences and perceptions related to high perceived ability and/or high procedural justice, much more discussion surrounded perceptions of low perceived ability and/or low procedural justice.

Despite the endorsement of at least one perception consistent with high perceived ability by 10 participants, there were substantially more instances of perceptions consistent with low perceived ability. Specifically, these participants indicated that they did not know who to go to or reported feeling that their voice would be heard or valued if they had a concern. In several cases this feeling stemmed from previous negative interactions with those in the IPV community. One provider recounted an experience from a discussion with various stakeholders when the standards were being developed, "I only remember two, maybe three discussions that were put out there but it became so unsafe that no one was willing to comment". Another participant described similar experiences, "A lot of politics has entered in. I'm willing to discuss anything with anybody but I want to discuss it. I don't want to be yelled and screamed at. And I don't want to be dismissed". Finally, one participant described their hesitation to discuss their views towards working with female batterers based on what he/she heard about one meeting in particular:

I talked about going into a meeting ... and the person there told me, 'they'll squash you, it's such a heated, fiery battle that they will just simply stomp all over you. You'll go out of there feeling like a crushed cockroach.' And so with that, I don't think I want to go to that meeting.

These participants reported holding back on sharing their views and opinions in order to avoid being placed in difficult positions. These views may or may not stem directly from actions of the Standards Advisory Committee, but the culture in the community appears 
to restrain some providers from taking their concerns to any relevant committees or individuals. Other perceptions consistent with low perceived ability included comments that indicated uncertainty surrounding possible participation or value of their opinions. For instance, when asked whether they believed their input would be valued, one provider said, "I'm not certain. I would hope so". Another participant was asked if they would know who to raise concerns with and if those concerns would be valued. They quickly replied, "No and no". Thus, some individuals appear to have had previous interactions that contribute to their lack of confidence regarding their ability to participate and be heard, while others lack of confidence may stem from limited exposure to the Standards Advisory Committee.

Low procedural justice was coded to capture participants' perceptions of unfairness. Most comments coded as low procedural justice centered around representativeness of the committee and how lack of diversity may impact the process by which standards were developed and their content. The belief that the Standards Advisory Committee does not represent most providers was discussed in the context of race/ethnicity, sexual orientation, class, role in the community collaborative response, and geographic location of those participating on the Standards Advisory Committee. One participant raised concerns related lack of cultural diversity:

I don't think it's a very diverse group. I think that there's a somewhat diverse representation of disciplines but that's not the same as having people from tribes, people from the Hispanic community, people from Europe. In this state a large percentage of people come over... and they don't speak much English... those refugees, immigrant populations, they're not represented, the African American community isn't represented. 
Another participant spoke to the lack of diversity surrounding sexual orientation, "I don't think that the LGBTQ community was even represented. Same sex wasn't represented". Other participants mentioned the lack of representation from non-metropolitan areas, “I'm not saying that they're purposefully trying to exclude folks from rural communities, I'm just saying it's more difficult and there's not much consideration for somebody that's outside the community. It's what's best for the majority". This participant is pointing out that while lack of inclusion of rural providers may not be intentional, the Standards Advisory Committee does not include adequate representation of rural communities. Other participants described similar concerns, “... there was not as much representation from rural providers" and "... it was more the major tri-county areas that had some access to resources that the rural communities don't [have]". The result of limited inclusion of rural providers was summarized by one participant, “[Standards are] something that was thought up where there's more people, more resources". In addition to cultural and geographic representation, one participant discussed the lack of representation from people of different socioeconomic class backgrounds, "It's majority culture, middle class, professionals". This participant indicated that because of this, the standards are best suited for program participants who are part of the majority, middle class culture, rather than inclusive of different experiences. One participant succinctly described the lack of cultural and geographic diversity in the committee, “... [there is] very little actual concern about what it's like to be from a disenfranchised community, what it's like to be from rural Oregon, what it's like to be from a community of color". Another area of concern voiced by participants was the profession of those on the 
Standards Advisory Committee, including how those professions translate to power on the committee. Two participants discussed the powerful role of corrections on the Standards Advisory Committee, "probation and parole has a big voice" and "community corrections... kind of co-opted the committee in some ways to promote their own agenda". The dominant role of community corrections appeared to make some participants worried that the standards may make batterers intervention more focused on its role in the criminal justice system as opposed to its role as a treatment. While participants were weary of the power in the hands of community corrections, they also raised concerns about the lack of representation from judges, lawyers, and law enforcement, “...we did not have as many attorneys or judges as we would have liked". Similarly, “... one of the things we were lacking was real representation from judicial and law enforcement... that could've really helped". One participant described why they felt the lack of individuals from various fields was problematic:

I think sometimes when people are making decisions around what batterer intervention providers should be doing when they don't even know what it looks like. They don't even understand the struggles that we deal with fully. Or to even realize that these men are human.

This participant believe that at times, representatives from the criminal justice system are uneducated about how BIPs function or do not believe that men participating in the BIPs are capable of change. They indicated that the lack of knowledge and confidence may impact how the standards are developed. These comments point to the numerous concerns participants had regarding the representativeness of the Standards Advisory Committee, as well as the impact this may have had on standards. 


\section{Section 3: Negative Attitude Change and Maintenance}

In order to examine whether those who initially viewed the standards as negative shifted their attitudes or maintained their attitudes, two pairs of codes were utilized. First, it was necessary to establish participants' initial response to the state standards. Initial responses were coded as either positive or negative based on the content of their experiences. Positive initial response $(\boldsymbol{\kappa}=.64)$ was coded when participants indicated an initial overall agreement with the standards, initial perception of limited impact, and initial agreement with specific components of the standards. Negative initial response (к $=.86)$ was coded when participants indicated an initial overall disagreement with the standards, initial perception of great impact, and initial disagreement with specific components of the standards. Across all participants positive initial response was coded 36 times and frequency ranged from $0-7$ instances per participant $(M=2.77, S D=$ 2.17). Nearly every participant $(n=12 ; 92 \%)$ reported at least one experience consistent with positive initial response. Across these 12 participants, this code was applied an average of $3.00(S D=2.09)$ times per participant. Negative initial response was coded 35 times and frequency ranged from $0-5$ instances per participant $(M=2.69, S D=1.55)$. Eleven participants (85\%) reported at least one experience consistent with negative initial response. When just these 11 participants were considered, negative initial response was coded in an average of $3.18(S D=1.08)$ instances per participant.

While two participants described experiences exclusively consistent with positive initial response and one participant described experiences exclusively consistent with negative initial response, most participants reported experiences of both positive initial 
response and negative initial response. To account for this, a positive initial response ratio was created. Specifically, the number of instances of positive initial response was divided by the number of instances of positive initial response and negative initial response combined. This process produced a ratio indicating the extent to which participants' experienced positive initial response, with zero corresponding to no mention of positive initial response and one corresponding to experiences of positive initial response exclusively. The average initial response ratio across participants was .52 (SD $=.31$; ranging from $0.00-1.00$ ), indicating $52 \%$ of their comments regarding their initial response to the standards were consistent with positive initial response. Five participants $(38 \%)$ reported a higher frequency of positive initial response experiences as compared to negative initial response experiences. Six participants (46\%) reported a higher frequency of negative initial response experiences as compared to positive initial response experiences. The remaining participants $(n=2 ; 15 \%)$ reported an equal frequency of positive initial response and negative initial response experiences.

This information highlights several features of participants' initial responses to the state standards. First, as for both actual and perceived control, most participants reported some indication of both a positive initial response and negative initial response. While most individuals had either a primarily positive or negative initial reaction, they reported experiences that included both positive and negative initial reactions to the standards. Second, it is evident that experiences of initial response varied across participants. On average, most comments made related to initial response were indicative of a positive initial response. This provides important insight in regards to the 
participants overall. Specifically, as a group it appears they initially viewed the standards positively. While this was the case, it is important to recognize that the majority of participants reported equally positive and negative, or a primarily negative initial response to the standards. Thus, those who had a primarily positive initial response were more vocal in describing their positive response, which has contributed to an average initial response ratio above .50 despite the fact that most participants' individual initial response ratios were .50 or lower. This reinforces the importance of examining participant responses in different ways as different vantage points may reveal unique trends. Next, the content of interview responses related to the shifting and maintenance of negative attitudes towards the standards were evaluated.

The code of positive initial response was applied when various types of experiences were described. Most participants who reported at least one comment consistent with positive initial response noted that initially they agreed with the concept of standards as a whole. One participant explained that the notion of standards seemed like a step forward for the field, "I felt like it was progress... for batterers intervention in Oregon". This view of the standards as progress for the field may be due to the credibility formal policy may provide. For instance, one participant explained, "I saw [the standards] as a form of validation... a little more teeth and recognition". While some initial agreement with the concept of standards was centered on progress and credibility, some participants appreciated having structure to develop their programs. Participants explained, "I was happy that there was some guidelines... we [shouldn't] wander into the wilderness without a map or compass" and "I was starting, so I was like, oh good, 
clarity". Thus, the standards appear to have provided desired structure for some providers that were in the midst of developing their programs. While some participants initially enjoyed the structure of the standards, others indicated they appreciated that the standards initially functioned as guidelines rather than strictly enforced rules. As one provider explained, "At that time, by and large, I thought it was not too bad. There was still a lot of freedom in the approach. There was still quite a bit of leeway at first". In addition to overall initial agreement, the positive initial response code was also applied when participants noted that the standards did not have a large impact on their program initially. Seven participants indicated that initially the standards did not have a large impact on their programs. Some of these participants referenced specific requirements, while others discussed impact more broadly. For instance, "I think in content we were pretty much all in agreement on the things that needed to be covered, so there was no surprise with any of that". Another participant described, "I came in with somebody who was [on the committee] and that's how I got trained and how I learned. So, I didn't have to change anything because it was already there". These descriptions highlight the fact that for some individuals, the initial set of standards closely corresponded to their current practice and therefore were not problematic to adopt. Finally, initial positive response also captured experiences in which participants noted initial agreement with specific components of the standards. For instance, one participant described specific components of the standards that they felt positively towards, "It seemed to come from a good place... victim safety, incorporating community involvement...".While most providers described 
at least one experience consistent with initial positive response, nearly as many described experiences consistent with a negative initial response to the standards.

The code of negative initial response was applied when participants described initial overall disagreement with the standards, perception that the standards greatly impacted their program initially, or initial disagreement with specific components of the standards. Of the 11 participants who described at least one experience consistent with negative initial response, only three participants described initial overall disagreement. Each of these participants raised a concern about why they believed the use of standards was unnecessary or problematic. One participant described the standards as premature, "I thought it was a mistake. We don't know enough". The second participant was concerned about how restricting clinical judgment may impact victims safety, "I was initially worried, how many people are we gonna get killed over this?". The third participant voiced concern over the role of government in providing intervention services, "One of the initial concerns was... you put a government entity in charge, specifically an entity that has the power and control system in place, and it's a disaster". For these participants, the introduction of the standards was met with global concerns about what this would mean for the future of BIP services, including innovation in the field and victim safety. While only three participants discussed overall disagreement, eight participants reported that the standards substantially impacted their program when they were introduced. Many of these participants were fearful about how the standards would be monitored and enforced. As one participant described in upbeat and confident manner, "All of it was so new. I just think it was a little scary and you know I understood the importance of it... we 
were going to do everything we could to follow it". Another participant explained a similar fear:

I felt like I was going to be in trouble. I felt that... big brother was going to come in and tell me I couldn't do things the way that they were effective for the people I was working with.

Thus, the initial introduction of the standards caused fear and anxiety for some providers who wondered how they would make the necessary changes and what types of consequences would arise if they were not able to comply with all components.

Other participants noted specific program characteristics that were immediately impacted by standards. Specific requirements that programs initially had to change included the use of male and female co-facilitators, victim advocacy and BIP training for facilitators and language in program documents. One participant explained how the creation of the standards motivated him or her to seek resources to achieve the requirements of the standards, "The first thing I did was take [the standards] to my boss and say, we got to do things different. We need to get [another facilitator] in here, I need training". Thus, for some the standards had a substantial initial impact and motivated them to start making changes to the components, which were not in alignment with the standards.

Participants were also coded as having a negative initial response when they described initial disagreement with specific components of the standards. Seven participants listed at least one component of the standards that they did not agree with when they first learned of the standards. These components included the review of policies and procedures by a victim advocacy organization, the gender specific focus of 
the standards, the requirement surrounding male-female co-facilitation, program length, lack of individualized treatment, and the prohibition of couples or family therapy. These experiences suggest that many providers had at least some reservations when the standards were introduced. For some this was a global disagreement or fear, while others felt negatively about specific components of the standards.

Next, participants' current perception of the standards was examined through the use of two codes, positive current response and negative current response. Positive current response was coded $(\kappa=.76)$ when participants reported experiences of current overall agreement with standards and similarities between the standards and current or ideal practice. Negative current response was coded $(\kappa=.63)$ when participants reported experiences of overall disagreement with the standards and discrepancies between the standards and current or ideal practice. Across all participants, positive current response was coded 116 times and frequency ranged from $3-12$ instances per participants. All 13 participants described at least one experience consistent with both positive and negative current response $(M=8.92, S D=2.47)$. Negative current response was coded 179 times and ranged from $4-44$ instances per participant. This code was applied to at least one comment in all 13 participants' interviews $(M=13.77, S D=10.19)$. Most participants $(n$ $=11 ; 85 \%$ ) described 16 or fewer instances of negative current response, but two participants voiced substantially more instances of negative current response. Specifically, one participant described 21 and another participant listed 44 instances indicative of a negative current response. 
To account for participants' experiences of both positive current response and negative current response, a current response ratio was created. Specifically, the number of instances of positive current response was divided by the number of instances of positive current response and negative current response combined. This process produced a ratio that indicates the extent to which participants experienced a positive current response, with zero indicating no mention of a positive current response and 1.00 indicating experiences of a positive current response exclusively. On average, participants had a current response ratio of $.44(S D=.15$; ranging from $.06-.67)$, indicating that on average $44 \%$ of the participants' comments related to current response were indicative of a positive current response. Four participants $(31 \%)$ reported a higher frequency of positive current response experiences as compared to negative current response experiences. The remaining participants $(n=9,69 \%)$ reported a higher frequency of negative current response experiences as compared to positive current response. Thus, most participants reported primarily negative current experiences with and perceptions of the standards. Next, the content of material coded as negative current response or positive current response was assessed.

The negative current response code was employed in instances where participants indicated disagreement with the standards overall, or misalignment with the components of standards and current or ideal practice. Seven participants mentioned overall disagreement with standards. Each of these participants described the standards as either too rigid or not inclusive of program or participant diversity. Many of these comments were centered on the idea that once a policy is put in place, it is very difficult to modify 
the policy or allow the policy to evolve with the field. As one participant observed, "When you put things into policy it's... not a breathing document" and another noted, "I think that [the negative aspect of state standards] is putting something in stone that can't be malleable and change into what is an inherently changing process". Further, one participant described, “The approach to standards is very simplistic and I think that the problem and the change of the problem is a... much more complex answer". These participants felt strongly that while standards may be positive in some respects, if they cannot evolve with the field they will always be flawed.

Negative current response also was found in participants' discussion of program diversity, specifically diversity related to geography, resources, and clientele. One participant indicated that the standards do not account for the experience of programs in more remote areas, "It would be more helpful if those standards were a little bit more reflective of the broader state and not just the metropolitan areas and not just the majority culture". In terms of diversity in clientele, one participant discussed the standards' lack of relevance for female perpetrators, "People are people and any of us can be abusive and the standards are not set up that way. They standards are not set up that way at all. The standards are only set up for males". Thus, some participants felt that in a general sense, the standards need to be more inclusive of different types of experiences.

In addition to overall response to the standards, misalignment with specific components of the standards was considered. All 13 programs listed specific components of the standards with which they disagreed. Specific components participants reported misalignment with include program length, lack of certification or licensing, male-female 
co-facilitation, the lack of discussion about appropriate program fees, victim contact policies, facilitator training requirements, program completion requirements, the nontherapeutic approach endorsed by the standards, prohibition of couples and family counseling, the mandate that a victim advocacy agency review program materials, aftercare requirement, the definition of the local supervising authority, allowance for former program participants to become facilitators, and the lack of value for clinical judgment.

The positive current response code was utilized to capture participant's current agreement and alignment with the standards. Twelve participants described current overall agreement with the standards. This sentiment was discussed as an agreement with the concept of standards or dialogue about the value of the standards. For instance, participants indicated, "I think it's important to have some kind of uniform criteria for these kinds of programs", and "I feel it's important that there are some guidelines to operate by to make sure that we are... treating the batterers to the best of our ability and things aren't just willy nilly". This comment reflects a general sense among participants that the idea of standards overall is viewed in a positive light. When asked whether components of the standards align with program practices or ideal practices all 13 participants named at least one component of the standards that is in alignment with their current or ideal practices. Specific components participants mentioned include provisions related to victim safety, use of a coordinated community response to IPV, male-female co-facilitation, staff training requirements, prohibition of anger management and couples counseling, requirement that policies and procedures are written, emphasis on 
accountability and development of an accountability plan, program length, the requirement for aftercare, and confidentiality requirements. It appears that participants are able to identify aspects of their program practices that align with the standards, as well as components of the standards that are in accordance with their views of ideal program practice. While voicing at least some current agreement and positive view of the standards occurred across all participants, every participant also described components of the standards with which they do not agree.

It is interesting to note that many of the components of standards raised in relation to a negative current response were also discussed in relation to a positive current response. This discrepancy highlights the lack of consistency or uniformity of opinion across participants. Some participants viewed the inclusion of requirements such as malefemale co-facilitation and prohibition of couples counseling as positive components of the standards, while others felt that this was a negative component of the standards. After assessing participants' initial and current response to the standards, this information was utilized to address $R Q 2 c$ and $R Q 2 d$.

\section{Section 4: Absoluteness}

In order to address these hypotheses, two codes were utilized. Absoluteness $(\kappa=$ .62) was coded when participants indicated that they believe compliance is required or expected, that others value compliance (e.g., corrections, victim advocates, etc.), or they have had a referral source change due to compliance. Non-absoluteness $(\kappa=.66)$ was coded when participants indicated that they believe compliance is not required or expected, or that others do not value compliance. The absoluteness code was applied 77 
times and the frequency of this code ranged from 0 to 24 instances per participant $(M=$ 5.92, $S D=6.82)$. Twelve of the 13 participants $(92 \%)$ made at least one comment indicative of absoluteness. Among these 12 participants, statements reflecting absoluteness were made an average of $6.42(S D=6.88)$ times. The non-absoluteness code was applied 48 times and the frequency of this code ranged from 0 to 9 instances per participant $(M=3.69, S D=3.11)$. Eleven participants $(85 \%)$ voiced at least one statement that indicated perceptions of non-absoluteness. Among these 11 participants, non-absoluteness perceptions were described an average of $4.36(S D=2.91)$ times per participant.

To account for participants' perceptions of the standards as both absolute and non-absolute, a ratio of absoluteness was created. Specifically, the number of instances of absoluteness was divided by the number of instances of absoluteness and nonabsoluteness combined. This process produced a ratio indicating the extent to which participants perceived the standards as absolute, with zero indicating no mention of absoluteness and 1.00 indicating perceptions of absoluteness exclusively. Absoluteness ratios ranged from 0 to 1.00 . The average absoluteness ratio was $.58(S D=.33)$, indicating that on average $58 \%$ of comments related to absoluteness were related to the presence of absoluteness rather than it's absence (non-absoluteness).

Next, the content of codes utilized to gauge experiences of absoluteness were examined. Absoluteness was coded to capture descriptions of both perceptions and experiences indicating that the participant viewed the standards as a policy that should or must be followed, or that others value. These comments took three forms. First, most 
participants $(n=10)$ reported believing compliance was required, expected, or indicated that a client referral source had changed due to failure to comply with the standards. The strength of these beliefs varied widely across participants with some participants speaking broadly, others describing incorrect possible outcomes (e.g., outcomes not actually included in the standards), and one participant describing a loss of business due to the standards. Participants reported perceptions that non-compliance would have extreme consequences for their program. For example, when asked what would happen if they did not comply with the standards, one participant reported, "You don't get to practice, you don't get to serve. You don't get to get referrals. You're not trusted, you're blackballed". Another reported a similar sentiment, "We all know if we don't comply to these standards then we don't get referrals". One provider noted that they believe compliance is necessary to received referrals due to the perception that referral agencies are in a position of power, "In order to stay in business, you have to comply. [Referral sources] have the power. They really have the power, we don't have the power as batterer intervention providers". Thus, many participants believe that if they were to be significantly out of compliance, they would lose their referrals and subsequently have to stop providing services. While this consequence is not explicitly stated in the standards, these participants rightly perceive that those making referrals are supported to avoid referrals to programs that are noncompliant with standards. Other participants described extreme outcomes that are not actually outlined in the standards. For instance, "I imagine there would be legislative consequences in the form of however those consequences are doled out. You know, as far as fines or whatever for not complying". Similarly, "We've 
been taught that there's a rule. It's like the IRS, you don't know exactly what... but you know they are going to do something". Even more extreme, one provider reported they are hesitant to provide services due to what they believe to be possible outcomes of noncompliance, "I'm out of compliance and I have no desire to go to prison or be fined because I'm out of compliance". The belief that fines or criminal sanctions could be applied for non-compliance is not supported by the content of the standards. This discrepancy highlights the lack of understanding regarding the consequences of noncompliance and how this misunderstanding may lead to extreme perceptions and fear for some participants.

In addition to whether there is an expectation of compliance, when asked whether participants were aware of any agencies that value compliance, nine participants named at least one agency they believe values compliance. Specific agencies mentioned include probation, the local supervisory agency, the local domestic violence council, victim advocacy agencies, other BIPs, the Department of Human Services, and judges. The breadth of agencies named, as well as the fact that most participants named at least one agency, reinforces the notion that most participants believe that members of the community collaborative response view compliance with the standards as important. While many participants indicated compliance is expected or that other agencies involved in the response to IPV value compliance, only one participant discussed changes in referrals that they attribute to the standards. This participant indicated that their program has struggled with compliance and they believe this has led to a substantial decrease in referrals. As they describe, "The standards have, in effect, driven me out of business". 
Thus, while only one participant indicated compliance has directly impacted their referrals, this impact appears to be substantial. Additionally, it is worth noting that no participants reported an increase in referrals related to compliance with standards.

Through reviewing participants' comments related to absoluteness, it is clear that regardless of the accuracy of the perceived consequences for non-compliance, most participants view compliance as expected or necessary in some form. These participants varied in the accuracy of their beliefs, with some providers believing incorrectly that certain consequences can stem from non-compliance (e.g., fines or jail). Further, most providers named at least one agency that they believe values compliance. Those named were diverse and included agencies related to victim advocacy, law enforcement, peers in the BIP community, and health services. Additionally, one participant noted that lack of compliance with the standards has had a profound impact on the referrals they receive and this has greatly influenced their program's viability. While most participants described experiences and perceptions consistent with absoluteness, participants also discussed ways in which the standards were not viewed as expected or required.

Non-absoluteness was coded to capture perceptions of compliance with standards as not expected, required, or valued. Throughout the interviews, 10 participants indicated that compliance with the standards is not expected or required at least once. Some participants described that they are unaware of consequences and therefore question whether consequences exist. For example, when asked about consequences for noncompliance one participant reported, “I don't think there are any. That's my understanding. Maybe I'm not right but to my knowledge, nothing". In addition to beliefs 
that adherence to the standards is not expected or necessary, one participant indicated the lack of absoluteness regarding compliance changes their priorities. This participant noted, “There's no standards that say if you don't do it we're shutting you down. So, I've got other priorities". This participant in particular was responsible for providing several types of services at their agency and because compliance with standards is not formally enforced, they chose to place their attention on the fields that do require certification (e.g., drug and alcohol service provision). Another participant who is familiar with the standards creation process provided insight into the lack of formal monitoring:

In the state standards there is no mechanism... if an agency is not doing [the standards]. And there is no mechanism if a county is not following these standards... My understanding is that the reason they did not put any of those mechanisms in place is because there was no money.

This participant highlights the fact that in addition to the lack of formal statewide monitoring of compliance, this lack of enforcement has contributed to some local areas adopting their own regulations for BIPs. Participants were also asked to describe the extent to which others value compliance with standards. The impact of this lack of enforcement as it relates to local standards was observed when participants discussed whether others value the state standards.

Three participants described that in their county, state standards are not valued and instead they are expected to adhere to local standards. As one participant described, “The judges and their probation officers in [specific county] don't care about the standards so they decided they were going to do things differently....that gives other 
people permission to say well, we don't need to do it either'. Another participant reported that because local standards have been adopted, compliance with state standards would be problematic for their referrals, "Our local county expects us to comply with their version of the standards. [If we followed state standards] they wouldn't refer to us". Thus, for participants in locations with local standards, participants perceive that it may be harmful to the program to adhere to state standards. Instead, if they would like to continue providing services, meeting local requirements becomes more pressing. The third participant who discussed the lack of value for standards in some areas reported that those in positions of power in their area have used that power to overrule what the state has prescribed. Specifically, when asked about state standards this participant indicated, "[Specific county] could care less. Their attitude is pretty much we get to do what we want no matter what the state says. They say it out loud and they don't care". Together, these three participants emphasize the complexity and ambiguity surrounding absoluteness when different agencies in power have different expectations. In addition to some participants perceiving certain counties as not valuing standards, an additional two participants indicated there are instances when judges or lawyers do not value the standards. One participant described instances that despite awareness of the standards, judges made decisions contrary to the recommendations of the standards. This participant recalled, "There were times when various judges would say, no, we don't want them to do [the length] of the standards, we want them to do less than the standards". In addition to perceptions that standards are not valued due to those in power making their own decisions, one participant also explained that because there are so few programs in their 
area, having a program to send clients to becomes more important than compliance. This participant explained, "Do they value standards? No. Well, corrections, no, the courts, no.... I think they just send to whatever's there". While lack of value for standards related to non-absoluteness was primarily discussed in a negative light, one participant described the lack of value surrounding compliance in a positive way. This provider believed that the lack of value for standards they have observed from corrections gives power to the provider to make decisions. Specifically, "I've gotten encouragement from corrections to do what I want to do because they think it's the right thing to do". This points to the fact that lack of value and enforcement may be seen as beneficial for some and problematic for others.

Participants' perceptions related to non-absoluteness highlight several key features of their views towards standards. First, it appears that most participants have at least a vague sense that standards are not formally enforced or monitored by a statewide agency. This lack of enforcement and monitoring may impact how some participants run their programs. Specifically, because other types of services may require formal certification while BIP services do not, meeting requirements for BIPs may be near the bottom of their priority list. Second, the lack of enforcement and monitoring has extended to local entities creating their own standards that are different than those put forth by the state. The message this appears to send to providers is that the state standards do not have to be followed and instead whoever is in power locally can modify requirements as they see fit. Finally, a small number of participants described other entities that do not value compliance, including judges and corrections. For some this was seen as problematic as 
judges may make individual decisions regarding clients, but at least one participant appreciated the flexibility that lack of value provided them to make decisions about their program.

It is also valuable to compare experiences of absoluteness and non-absoluteness to better understand how these experiences differ. One major theme is that most participants described the standards in terms of both absoluteness and non-absoluteness. While this is true, the reference point for these perceptions appears to differ. Specifically, some individuals discussed absoluteness in terms of the perceived impact of noncompliance on their referral sources and discussed non-absoluteness in reference to the state taking action in cases of non-compliance. The reverse was also true -- some participants reported fear of extreme consequences in terms of the state, but described the standards as not valued in their local environment. These perceptions of both absoluteness and non-absoluteness may cause confusion for participants who must decide to which entities they should be most responsive. For example, while some participants fear consequences from the state, if they believe the standards are not valued by their referral source, they may chose to align with the expectations of the referral source despite apprehension due to possible consequences from the state. While perceptions of expected or required compliance vary, most participants were able to list at least one agency that they believe values compliance. Even participants who indicated that local standards have been enacted reported that some agencies (e.g., victim advocacy agencies) supported and valued the state standards as opposed to local standards. Together, this information indicates that participants tended to view the standards as indicative of 
absoluteness as opposed to non-absoluteness, but the content of these perceptions and experiences varied based on local context and knowledge. All of this information was utilized to determine whether the construct of absoluteness, as operationalized, differentiates those who changed and maintained negative attitudes towards the standards.

\section{Section 5: Legitimacy}

In order to address this research question three aspects of legitimacy were assessed in the interviews. These components included procedural justice, norms, and policy logic. To capture experiences and perceptions consistent with these three aspects of legitimacy, three pairs of codes were utilized. These codes included the codes of high procedural justice and low procedural justice, positive norms and negative norms, and high policy logic and low policy logic.

Procedural justice. Experiences consistent with high procedural justice and low procedural justice were previously discussed in the context of perceived control. The information gathered from this code was applied once more in the context of legitimacy. To summarize, high procedural justice $(\kappa=.66)$ was coded when participants described the process by which standards were created as fair or the committee as representative. The high procedural justice code was used 12 times with frequency ranging from $0-5$ instances per participant $(M=.92, S D=1.55)$. Only 5 participants $(38 \%)$ reported at least one experience consistent with high procedural justice. The code of low procedural justice $(\kappa=.55)$ was utilized when participants reported perceiving the standards process as unfair or the committee as unrepresentative. This code was applied a total of 32 times, 
with frequency ranging from $0-7$ instances per participant $(M=2.46, S D=2.54)$. Nine participants $(69 \%)$ reported at least one experience consistent with low procedural justice.

In order to account for experiences of both high procedural justice and low procedural justice simultaneously, a procedural justice ratio was created. In order to create the procedural justice ratio, the number of instances of high procedural justice was divided by the number of instances of high procedural justice and low procedural justice combined. This process produced a ratio that indicates the extent to which participants perceived the standards as being created with procedural justice, with zero indicating no mention of high procedural justice and 1.00 indicating perceptions of high procedural justice exclusively. The average procedural justice ratio was $.30(S D=.25$; ranging from $0.00-.67$ ), indicating that on average $30 \%$ of comments made regarding procedural justice were indicative of high procedural justice. It is important to note that four participants did not report experiences consistent with high procedural justice or low procedural justice. When only the nine participants who did receive codes of either high procedural justice or low procedural justice were examined, the average procedural justice ratio was $.21(S D=.25)$. This indicates that for those that mentioned either high procedural justice or low procedural justice at least once, only $21 \%$ of their comments related to procedural justice were indicative of high procedural justice. Further, of the nine participants, only one (11\%) reported a higher frequency of high procedural justice perceptions as compared to low procedural justice perceptions. The remaining participants $(\mathrm{n}=8 ; 89 \%)$ reported a higher frequency of low procedural justice 
perceptions as compared to high procedural justice perceptions. Thus, most participants viewed the creation of standards as low in procedural justice. In addition to this, norms and policy logic were also assessed in order to develop a comprehensive view of legitimacy.

Norms. Positive norms was coded $(\kappa=.88)$ when participants described positive discussions about standards in the community. Negative norms was coded $(\kappa=.65)$ when participants described negative discussions about standards in the community. Across all participants positive norms was coded eight times and frequency ranged from $0-2$ instances per participant $(M=.62, S D=.77)$. Slightly less than half of the participants $(n$ $=6 ; 46 \%$ ) reported at least one experience indicative of positive norms. When only these six participants are considered, positive norms were discussed an average of 1.33 (SD= .52) times per participant. Negative norms was coded 12 times and frequency ranged from $0-4$ instances per participant $(M=.92, S D=1.38)$. Less than half of the participants $(n=5,38 \%)$ reported at least one experience indicative of negative norms. When just these five participants are considered, negative norms were discussed an average of $2.40(S D=1.14)$ times per participant.

Next, in order to account for participants' discussion of both positive norms and negative norms, a norms ratio was created. In order to accomplish this, the number of instances of the positive norms code was divided by the number of instances of positive norms and negative norms combined. This process produced a ratio that indicates the extent to which participants perceived the standards as being viewed positively in the community, with zero indicating no mention of positive norms and 1.00 indicating 
perceptions of positive norms exclusively. The average norms ratio was .47 ( $S D=.39$; ranging from $0.00-1.00$ ), indicating that on average $47 \%$ of the comments made related to norms were indicative of positive norms. It is important to note that six participants did not report experiences consistent with positive norms or negative norms. When only the seven participants who discussed norms were examined, the average norm ratio was .48 $(S D=.27)$. Less than half of these seven participants $(n=3 ; 43 \%)$ reported a higher frequency of positive norm perceptions as compared to negative norm perceptions. The remaining participants $(n=4 ; 57 \%)$ reported a higher frequency of negative norm perceptions as compared to positive norm perceptions. These findings indicate that while the valence of norms was infrequently discussed, more participants mentioned at least one experience consistent with positive norms, while there were more instances of experiences related to negative norms across the sample.

The discussion of positive norms in the community regarding standards typically included statements indicating the standards were discussed favorably by others or that others did not have concerns about the standards. For instance, one participant noted, "As far as the [local BIP providers] meeting, there was a lot of consensus [with the standards]". Other participants reinforced this notion that the standards were not viewed as problematic, "There weren't any criticisms" and "What I've seen is that everybody's pretty on board with it". These responses indicate that positive norms were generally in the context of not having concerns with the standards, rather than indications that those in the community viewed them positively. 
The discussion of negative norms included descriptions of providers discussing the standards as problematic or negative. Participants indicated that they had discussions with others in the community about the standards and they believed the consensus was one of concern. For instance, one participant noted, "I really don't know anybody who is in favor of the standards the way they are written". Another participant stated, "I think people are concerned overall". These participants perceived others to be unhappy with specific components of the standards, or the standards overall. Additionally, disagreement in the community surrounding anticipated changes to the standards was mentioned. During the time the interviews took place, the program length requirement was in the process of being revised. Three participants indicated that they have observed negative discussion surrounding this change. For example, "We were all on the same page. We're frustrated because it's like... they' re fixing something that wasn't broken in our opinion". Another participant reinforced this, "They were foolish to change [the program length requirement]... I think we all pretty much feel the same way about it... the general consensus was it was alarming to a lot of us". Thus, it appears that the higher prevalence of negative norms may be impacted as the current context changes.

Community discussion. While only positive norms and negative norms were relevant to the examination of legitimacy, given the relative infrequency of these codes, the extent to which discussions more generally occurred in the community was also examined. Specifically, two codes were designed to capture discussion that was neither positive nor negative, or lack of discussion. Limited contact $(\kappa=.71)$ was coded when participants indicated they were not in touch with other providers. This code was 
included to determine whether participants perceived themselves as part of a community of BIPs. This code was applied a total of six times, with frequency ranging from $0-2$ instances per participant. On average, this code was applied $.46(S D=.78)$ times per participant. Four participants $(31 \%)$ made at least one comment indicating they are not heavily involved in the BIP community. Among only these four participants, limited contact was coded an average of $1.50(S D=.58)$ times. These participants indicated that they did not have strong relationships with other providers. One participant described, "I don't know anyone who does treatment except for the [people] who work with me doing it. Which I don't like... I'd like to be more involved'. Another participant echoed this sentiment, "I wish I was more [involved]. I would love to be in the loop more, but I'm not". A third participant remarked, "I feel pretty much alone". Thus, it appears that some participants do not have a great deal of contact with other providers. This may partially explain why only seven participants described perceptions of positive or negative norms. Another explanation for the lack of positive and negative norms may stem from the fact that discussion in the community may not take an overtly positive or negative form.

After the process of familiarization with the interview transcripts was performed, a code was developed to account for reports of discussion regarding the standards that did not appear to be either positive or negative in nature. The discussion code $(\kappa=.58)$ captured experiences of discussion related to standards in the community in which participants did not indicate the valence of the discussion. This code was designed to assess the extent to which the standards are discussed among providers. The discussion code was applied a total of 30 times, with frequency ranging from $0-7$ instances per 
participant. On average, discussion was coded $2.31(S D=1.97)$ times per participant. Eleven participants $(85 \%)$ described involvement with a discussion about the standards at least once during their interview. When these 11 participants were considered in isolation, discussion was coded $2.73(S D=1.85)$ times per participant. The coded experiences included participation in or hearing of discussion relating to the different components of standards, though they did not feel that the conversations was exclusively negative or positive in nature. Participants reported discussion about training, program length, certification of providers, and aftercare. Additionally, participants reported discussion as a form of education. For instance:

People have a lot of questions about what's in them...especially the new people. So [discussion] is mostly just going over basic things about what's required or not required, or what I am supposed to be doing or not doing. It's not about this is bad or this is good, it's this is what [the standards include].

Others mentioned where they had participated in conversations about the standards. These settings included BIP provider meetings, DV council meetings and trainings. This information provides greater context for whether participants have a basis for identifying positive and negative norms in the community. It appears that most providers have discussed the standards with others at least once. Of the four participants that reported limited contact with other providers, two had been involved in at least one discussion about standards in the community. The remaining two participants did not report having any contact with other providers. While most participants have had some contact with each other, they may not have had enough exposure to identify the valence of perceptions towards the standards. While this is the case, the seven participants who reported positive 
norms or negative norms towards the standards in the community helps shed light on the extent to which providers view the standards as legitimate.

Policy logic. The final pair of codes utilized to determine perceptions of legitimacy included high policy logic and low policy logic. High policy logic $(\kappa=.77)$ was coded when participants indicated that they believed the standards were evidencebased or based on best practice. Low policy logic $(\kappa=.82)$ was coded when participants indicated they believed that the standards were not evidence-based or based on best practice. The code of high policy logic was utilized 20 times, with frequency ranging from $0-4$ instances per participant $(M=1.54, S D=1.33)$. Ten participants $(77 \%)$ made at least one comment consistent with the high policy logic code. When just these 10 participants were considered, the high policy logic code was applied an average of 2.00 $(S D=1.16)$ times per participant. The code of low policy logic was utilized 25 times, with frequency ranging from $0-9$ instances per participant $(M=1.92, S D=2.43)$. Ten participants $(77 \%)$ made at least one comment consistent with the low policy logic code. When just these 10 participants were considered, the low policy logic code was applied an average of $2.50(S D=2.51)$ times per participant. Only one participant did not make comments coded as either high policy logic or low policy logic, indicating the saliency of this dimension. These findings indicate that both high policy logic and low policy logic were evident in most participants' experiences and perceptions regarding the standards. Further, these codes were utilized at nearly the same rate, with usage ranging from 1.54 instances per participant for high policy logic and 1.92 instances per participant for low policy logic. 
To account for experiences of both high policy logic code and low policy logic, a ratio of policy logic was calculated. This was achieved by identifying the number of instances of the high policy logic code and dividing it by the number of instances of the high policy logic code and low policy logic code combined. This process produced a ratio that indicates the extent to which participants perceived logic in the policy of standards, with zero indicating no mention of high policy logic and 1.00 indicating perceptions of high policy logic exclusively. Policy logic ratios ranged from 0 to 1.00 . The average policy logic ratio was $.48(S D=.31)$, indicating that on average $48 \%$ of comments related to policy logic were indicative of high policy logic. It is important to note that one participant did not report experiences consistent with high policy logic or low policy logic. When only the 12 participants who reported at least one experience or perception of policy logic were examined, the average policy logic ratio was the same $(M=.48, S D$ $=.32$ ). Of the 12 participants, four (33\%) reported a higher frequency of high policy logic perceptions as compared to low policy logic perceptions, five (38\%) reported a higher frequency of low policy logic perceptions as compared to high policy logic perceptions, and three $(25 \%)$ reported an equal number of high policy logic and low policy logic perceptions. These findings indicate that providers had varied views regarding the extent to which the standards had policy logic. Next, interview material coded as high policy logic and low policy logic was examined.

First, interview content coded as high policy logic was examined. This code was utilized to determine the extent to which participants believe the standards are based in evidence or best practice. Four participants indicated that they believe the standards are at 
least somewhat evidence-based, though these participants also tended to make caveats when describing the standards as evidence-based. For instance, one participant indicated that the standards were developed for the majority population and for those individuals they were appropriate. Specifically this participant noted that the standards are, “...based on the evidence that is available for the population." Similarly, a second participant noted, "I think [the standards were created from] evidence-based practice to what they considered the norm, European American male to female [violence]". These two participants appear to believe that while the standards are evidence-based, they are only evidence-based when applied to certain majority populations. The view that standards are based in evidence was also discussed more broadly. For example, one participant surmised, "I believe it's got to be evidence-based for the most part". More commonly ( $n$ $=10$ ), participants indicated they believe the standards were created based on best practices. These participants reported that the standards took what was known to be most effective practice in the field and incorporated those practices into the content of the regulations. For instance, one participant explained, "I would say [standards were developed based on] consensus. I think it was all on experience". Another expanded, "I believe [the standards] are based on best practices from other states. And what has appeared to have been working in other communities". These experiences point to the fact that most participants did not view the standards as evidence-based and those who did view the standards in this way typically indicated the evidence-based nature of the standards was particular to specific populations. More commonly, participant's believed 
the standards were informed by best practice in the field, including provider experiences and information from other areas.

Next, low policy logic, which was coded to determine the extent to which participants do not believe standards are based in evidence or best practice, was examined. Nine participants stated that they did not believe all or specific parts of the standards were based in evidence. Seven of these participants specifically mentioned that evidence-based practices are not possible due to a lack of available evidence-based practices in the field of batterer intervention. One participant explained, "I think we need to acknowledge that evidence-based practice for batterers is not very mature. It's very nascent... we don't know what we don't know. We're learning as we go". Another participant called for research in order to establish evidence-based practices in this arena, "I think what was lacking [when standards were developed] and ... is still very lacking is research". Of the nine participants who noted the standards are not based in evidence, only one indicated this was a choice and not due to the lack of research in this area. This participant noted, “They keep trying to refine them and they don't refine them around research. They refine them around emotions... they were very, very much emotion based and still remain emotion based". Thus, while most participants agree that the standards are not based in evidence, the vast majority indicated that this was due to the state of the field rather than choices made by the Standards Advisory Committee. When participants were asked whether they believed the standards are based in best practice, only three participants indicated they do not agree that best practices were incorporated into the standards. These statements were largely consistent with those made regarding evidence- 
based practice. Namely, these participants indicated that best practice was not utilized because best practice has not yet been established. For instance, when asked about how the standards were developed, one participant described, "I don't think they are best practice because I don't think that there really was a best practice". Another participant reiterated this point, "We have to this date no idea what best practice is. No idea". The overall sentiment for those that endorsed low policy logic is that evidence-based practices were not incorporated into the standards because they do not yet exist. Additionally, a minority of participants believed this sentiment was true in terms of best practices. After each component of legitimacy was assessed, overall legitimacy of the standards was evaluated.

\section{Section 6: Relation of Standards to Program Closure}

Impact. High impact $(\kappa=.62)$ was coded when former providers indicated that their program stopped providing BIP services because components of the standards were unattainable, they disagreed with all or part of the standards, or their program viability (e.g., referrals) was impacted due to standards. Low impact $(\kappa=1.00)$ was coded when former providers indicated that their program stopped providing BIP services for reasons unrelated to the state standards. The high impact code was applied a total of eight times, with 0 - 5 instances per participant $(M=1.60, S D=2.07)$. Three participants made at least one statement indicating the standards had a high impact on their program closure. When just these participants are considered, high impact was coded an average of 2.67 $(S D=2.08)$ times per participant. The low impact code was applied a total of nine times 
with 1 - 3 instances per participant $(M=1.80, S D=.84)$. All five participants made at least one statement indicating the standards had a low impact on their program closure.

In order to account for participants reporting experiences consistent with both high impact and low impact, impact ratios were computed. This was achieved by identifying the number of instances of the high impact code and dividing it by the number of instances of the high impact code and low impact code combined. This process produced a ratio that indicates the extent to which participants perceived the standards impacted their program terminating BIP services, with zero indicating no mention of high impact and 1.00 indicating perceptions of high impact exclusively. Next, interview material coded as high impact and low impact was examined to determine how these perceptions and experiences were described by participants.

While three of the five former providers did indicate that the standards had a high impact on their decision to no longer offer BIP services, it is important to note that these experiences were discussed with caveats and coupled with other reasons BIP services were terminated. For example, one participant who made five statements indicating high impact was careful to note that while one part of the standards did play a role, it was just one factor. This participant explained:

The standards absolutely impacted my program but it was in combination with the environment. The standards and environment together made me stop providing services. I think if the environment were different I could have worked with the standards but I just couldn't push through the environment.

When this participant discusses the environment, they are referring to experiences of the culture of BIPs shifting: 
When I stopped providing services it was during a time when there was a movement towards mandating longer and longer programs. I felt many of the programs were too long, too expensive, and I didn't like their approaches. I think they used very directive and controlling behaviors. They put the clients down and were exhibiting the same behaviors we were trying to extinguish.

Thus, while this participant did feel the standards impacted their program closure, the standards were not responsible for the closure in isolation. Two other former providers indicated the standards had a high impact on no longer offering BIP services. These providers both indicated that while the standards played a role, it was the implications of the standards that were most important. One former provider explained:

Yes [the standards did have an impact] but more because of interpretation of the standards. Probation didn't feel I was taking victim safety seriously because I wasn't calling the victim to verify men's stories after each session. I tried to explain that this is problematic for victim safety and it is dangerous. It was the interpretation. I asked a judge about the standards and victim safety and they said what I did was in compliance but when we talked to probation they said it wasn't. So, we eventually had to shut down... it was incredibly frustrating to me.

For this provider, the ways in which the mandates included in the standards were interpreted by different agencies within the community collaborative response ultimately led to the removal of BIP services from their agency. Thus, while the standards played a role, it was ultimately a lack of information and education across referral agencies that led to the elimination of BIP services. Finally, the third participant who indicated the standards had a high impact on their program indicated that the actual mandates of the standards were not problematic in their view but the financial implications of the standards did play a role. Specifically, they commented:

The main reason [we stopped providing BIP services] was financial. Between all of the overhead costs like having two therapists, having them write so many reports, having them report to probation, going to [community meetings]... the 
bills just add up. People think we are raking in money doing these groups but with the overhead we are not making much at all.

For this participant, the actual content of the standards was acceptable but the costs associated with complying with the mandates proved to be problematic.

All five participants made at least one comment indicating the standards did not play a primary role in their program's decision to stop providing BIP services. Most of these participants indicated that the reason for no longer providing BIP services was logistical. Two participants explicitly named finances as the key rationale for ceasing BIP services. One participant explained, "Finances. Batterer intervention was extremely unpredictable in terms of caseload. I needed to have an affordable program so that men could actually attend but at the same time it was so unpredictable that having low fees was difficult". A second participant indicated components mandated by the standards impacted their finances but they did not stop services because of this:

We had to be part of the DV community because of the standards and so we had to pay for someone to got to those meetings. This expense impacted our finances and finances are why we stopped. But, to be honest, we would have gone to those meetings with out the standards. So no, they didn't make us close at all.

The remaining three participants noted that outside influences played a crucial role. One participant noted that a contract ended and other services became a priority:

I don't think the standards had anything to do with our program stopping BIP services. We do lots of different kinds of work here and so we started batterer intervention groups when we got a contract but once the contract was over we concentrated on other services.

A second participant described county climate and requirements as problematic:

[Our] county decided to go towards a more criminal justice punitive method of working with abusive people. I refused to go along with that and we lost all of our referrals... I met the state standards, every single state standard... it was 
probation that didn't like what I was doing, even though it was in line with standards.

The final participant noted how changes in immigration policy and enforcement were the key reasons for stopping services:

We were exclusively running Spanish speaking groups... around 2009 or 2010, referrals dropped because... ICE [Immigration and Customs Enforcement] started combing jails looking for anyone that had any violation and was undocumented... That then impacted the number of folks being arrested or detained for IPV, it went way down. People were less likely to call the police and report abuse because the outcome would likely be that their partner would be deported... our referral source just dried up.

Interest. During the process of familiarization, it became evident that one new code should be added to capture former providers' thoughts regarding reintroducing batterer intervention services into their programs. This code, interest $(\kappa=.62)$, captured instances where participants discussed whether they enjoyed providing batterer intervention services or indicated whether or not they would like to reintroduce services. The interest code was utilized four times, with frequency ranging from $0-2$ instances per participant. This code was applied an average of $.80(S D=.84)$ times per participant. Three participants $(60 \%)$ made at least one statement consistent with the interest code. Across these participants, the interest code was applied an average of $1.33(S D=.58)$ times per participant. Each statement regarding participant interest in batterer intervention services was positive, indicating that they enjoyed the work or would like to provide those services again in the future. One participant described their views on batterer intervention work, "I really enjoyed the BIP work... it was the most rewarding work I ever did. It takes time and it was hard, but it was rewarding”. Another participant reinforced these positive feelings towards the work, "I definitively did not stop because I 
didn't want to do the work anymore, I enjoyed the work". A third participant indicated their desire to provide these services again, "I would love the opportunity to do this work again. It was challenging and fulfilling".

Actual control. High actual control was coded a total of seven times, with frequency ranging from $0-5$ instances per participant $(M=1.20, S D=1.64)$. Three participants (60\%) made at least one comment consistent with high actual control and when just these participants were considered, the code was applied an average of 2.00 $(S D=1.73)$ times per participant. Low actual control was coded a total of 10 times, with frequency ranging from $0-3$ instances per participant $(M=1.80, S D=1.30)$. Four participants (80\%) made at least one comment consistent with low actual control and when just these participants were considered, the code was applied an average of 2.25 $(S D=.96)$ times per participant. In order to determine the proportion of high and low actual control codes, a ratio of actual control was created identically to its computation for current providers.

Experiences of high actual control included awareness of the process by which standards were created or awareness of key individuals. One participant reported that someone from their agency served on the original Governor's Committee, which contributed to the development of the standards, though they did not serve as a member of the Standards Advisory Committee. This participant was responsible for five of the seven comments related to high actual control. For instance, this participant reported, "Before the standards now, we knew about it when they were making the original standards because we had someone from our agency involved in that". The remaining 
two participants who described at least one comment related to high actual control reported knowledge of the creation process. For instance, "I know there was a committee that met and I know they had a hard time reaching consensus for what to put in [the standards]". Low actual control experiences included comments indicating the participant was not involved with or aware of the process or individuals involved in the creation of the standards. Most former provider participants reported that they had low actual control over the standards. For instance one participant reported, "I don't know how they were created. I just know they were given to us". Another participant reiterated this point, "I didn't play a role in creating the standards; at the point I started they were already in place". Thus, while one participant's program was heavily involved in the initial steps taken towards creation of the standards, most participants did not play an active role in their creation.

Negative attitude change and maintenance. In order to identify those who shifted and maintained negative attitudes towards the standards, two code pairs were utilized, positive and negative initial response, and positive and negative current response, as was done for current providers. Positive initial response was coded seven times, with frequency ranging from $0-3$ instances per participant $(M=1.40, S D=1.14)$. Four participants $(80 \%)$ reported at least one perception consistent with positive initial response and across these participants, the code was applied an average of $1.75(S D=$ .96) times. Negative initial response was coded four times, with frequency ranging from $0-2$ instances per participant $(M=.80, S D=1.10)$. Two participants $(40 \%)$ reported at least one perception consistent with negative initial response and across these 
participants, the code was applied an average of $2.00(S D=0.00)$ times. Initial response ratios were computed as was done with current provider participants. The average initial response ratio was $.67(S D=.47$; ranging from $0.00-1.00)$, indicating that on average, $67 \%$ of the comments related to initial response were indicative of a positive initial response. Three participants reported an exclusively positive initial response (initial response ratio $=1.00$ ), one participant reported an exclusively negative response (initial response ratio $=0$ ), and one participant reported a primarily negative initial response (initial response ratio $=.33$ ). Descriptively it appears that the average initial response ratio is approximately equivalent in the sample of former providers $(M=.67)$ as compared to current providers $(M=.52)$ and initial responses were primarily positive in both samples.

Comments coded as positive initial response included sentiments of overall agreement with standards, as well as specific components of the standards that participants agreed with initially. Former provider participants primarily indicated that they had positive sentiments towards the standards overall. For instance one participant noted, "I liked them and thought they were appropriate". Another voiced, "I understood and fully supported the rationale for the standards. I don't have any issues with how they were put together". Thus, when these participants first learned about the standards, they endorsed the overall scope and content. While this was the case for most former provider participants, two participants did report some aspect of a negative initial response. Comments coded as negative initial response included initial feelings of overall disagreement or disagreement with specific components. One participant described their 
initial response as an overall disagreement. Specifically, when asked to describe what they thought when they learned about the standards, this participant reported, "Negative. I was shocked. I thought they had crossed the line from being bad for the client to being bad for the community". The second participant had issues with more specific components of the standards. For instance, he or she noted:

When I saw the standards that passed in 2006, I was unhappy with some components of the standards. I especially didn't like that the curriculum has to focus on male entitlement and privilege and exclude things like impulse control, anger management, individual characteristics, and mental health... I was also unhappy that providers couldn't contact victims.

Thus, while a minority of participants viewed the standards as problematic initially, similar to current provider participants, the overall initial perception tended to be positive.

Current perception towards the standards was evaluated next. The positive current response code was utilized five times, with frequencies ranging from $0-2$ instances per participant $(M=1.00, S D=1.00)$. Three participants were coded as having a positive current response at least once. Across just these three participants, the code was utilized an average of $1.67(S D=.58)$ times. The negative current response code was utilized three times, with frequencies ranging from $0-2$ instances per participant $(M=.60, S D=$ .89). Two participants were coded as having a negative current response at least once. Across these two participants, the code was utilized an average of $1.50(S D=.71)$ times. Current response ratios were generated as was done for current provider participants. The average current response ratio was $.57(S D=.43$; ranging from $0.00-1.00)$. This indicates $57 \%$ of comments related to current response to the standards were indicative of 
a positive current response. It is important to note that one participant did not provide any information on their current response to the standards. When only the four participants who made at least one comment related to current response were considered, the average current response ratio was $.58(S D=.50)$. One participant reported an exclusively negative current response (current response ratio $=0$ ), two participants reported an exclusively positive current response (current response ratio $=1.00$ ), and one participant reported a primarily negative current response (current response ratio $=.33$ ). The current response ratios for former provider participants $(M=.57)$ were approximately equivalent to what was observed for the current provider participants $(M=.67)$. In both cases, on average, participants' comments were more aligned with positive current response as opposed to negative current response.

Comments indicative of a positive current response included those that indicated the provider felt positively about the standards overall or specific components of the standards when they last reviewed them. For instance, one participant described, "I liked the standards when I saw them and that didn't change. The way they were interpreted upset me but that was nothing about the standards. I think they are appropriate". Another participant reinforced this, "I always really liked [the standards]. I think they are important to have". Negative current response comments included comments indicating that components viewed negatively initially did not change. One participant explained, "The negative perception [about the standards] is still very much with me. Things may have changed but my impression has carried over". A second participant, who listed various components of the standards with which they initially disagreed, reported, "[My 
perception] really stayed stable. The same things that I didn't like at the beginning I didn't like later on". Thus, it appears that participants who had initial positive feelings towards the standards maintained positive feelings, while those who had initial negative feelings towards the standards maintained negative feelings.

Absoluteness. Former provider interviews were coded to capture perceptions consistent with absoluteness and non-absoluteness of the standards. The absoluteness code was utilized six times, with frequency ranging from $0-3$ instances per participant $(M=1.20, S D=1.30)$. Three participants $(60 \%)$ made at least one comment consistent with absoluteness and when just these participants were considered, the code was applied an average of $2.00(S D=1.00)$ times. The non-absoluteness code was utilized four times, with frequency ranging from $0-2$ instances per participant $(M=.80, S D=1.30)$. Three participants $(60 \%)$ made at least one comment consistent with non-absoluteness and when just these participants were considered, the code was applied an average of 1.33 $(S D=.58)$ times.

Experiences of absoluteness included comments indicating compliance with standards was expected or valued. One participant explained, "[Standards were] very clearly valued. Primarily by the courts. They asked about lots of different things related to the standards... For the most part, the places that gave us referrals, like probation and the courts, expected compliance". Another participant reiterated this, "It was definitely expected that we follow the standards...". Experiences of non-absoluteness included descriptions that compliance was not required or valued. For instance, one participant described: 
I didn't get the sense that compliance was required... I don't know who would even check to make sure I was following them. I guess I probably could have continued doing things my own way and ignoring the standards, but I didn't feel good about that.

A second participant echoed, "Judges often did not know about the standards unless they were primarily doing domestic violence cases. I don't think they really cared because they didn't know". A third participant indicated compliance with state standards was not required because their county had different requirements that had to be followed, "In [my county] we weren't expected to follow the standards at all. They expected providers to follow their rules but not the standards". These quotes point to the view of nonabsoluteness as the result of various experiences including the lack of enforcement body, lack of referral source knowledge about standards, and conflict with county requirements. These experiences were consistent with those described by current provider participants.

Legitimacy. Examination of legitimacy among former providers was limited. Specifically, no interview material was coded as either high or low procedural justice. Further, only one participant made one comment relevant to positive norms and no interview material was coded as negative norms. The one comment relevant to positive norms noted the positive perception of the standards, "Everyone viewed them very positively, we all thought some type of guideline was important". There were no additional comments made regarding norms about standards in the community. While participants did not describe experiences relevant to these two aspects of legitimacy, they did report perceptions related to one component of legitimacy, policy logic. High policy logic was only coded one time in one interview when a participant indicated believing the standards were based in evidence. This participant speculated, "I would assume the 
standards are evidence based but I guess I don't know for sure". More commonly, former providers indicated they did not believe the standards were evidence-based and these statements were coded as low policy logic. The code of low policy logic was applied five times and frequency ranged from $0-2$ instances per participant. On average, this code was applied $.53(S D=.51)$ time per participant. Four participants $(80 \%)$ voiced at least one comment consistent with low policy logic. When just these four participants were considered, the code was applied an average of $1.25(S D=.50)$ times. In the sample of former providers, participants either endorsed high or low policy logic exclusively. Specifically, one participant (20\%) was coded exclusively as high policy logic (policy logic ratio $=1.00)$, while the remaining participants $(n=4,80 \%)$ were coded exclusively as low policy logic (policy logic ratio $=0$ ). This differs from reports of policy logic in the current provider sample. The majority of providers in the current provider sample had policy logic ratios at .50 or above, indicating they primarily viewed the standards as consistent with high policy logic. Thus, the former provider participants appear to view the standards as lower in policy logic than the current provider participants. Responses were examined qualitatively to better understand policy logic for these participants and establish the nuances of their experiences.

The content of these codes focused on the lack of research and evidence regarding the content of standards. One participant explained:

The standards really aren't based on research. They are based on the orientation of the committee. I don't like that this led to such restrictive standards even when there isn't research to back up all of the different parts of it. 
This participant appears to believe the background of the Standards Advisory Committee members was the primary influence to the content of the standards. Another reinforces this sentiment but highlights that the lack of evidence-based practice in the field is at fault, "We don't know what works so we don’t have evidence-based practices yet". A third participant echoed, “I don't think they were [evidence-based]. Batterer intervention is really still in the infancy state. It's hard to know what best practice is because it's still changing as research comes along and we learn what is working". Thus, it appears that the content of material related to policy logic is similar to content coded for current provider participants. The one caveat is that while current providers discussed best practice, former providers were more exclusively focused on the lack of evidence-based practices and the standards.

\section{Section 7: Implementation Strategies}

The implementation strategies code $(\kappa=.62)$ was applied when participants described specific steps or strategies utilized to comply with the standards. Almost every participant $(n=12 ; 92 \%)$ described at least one implementation strategy. When just these 12 participants were considered, implementation strategy was coded an average of 4.75 $(S D=2.86)$ times.

The most commonly reported implementation strategy was the shifting of practices or policies in order to comply with the standards. Seven participants (54\%) described at least one practice or policy that was shifted as a direct result of the standards. These changes included the modification of program length to make programs longer, aftercare, putting policies and procedures in writing, confidentiality policies, victim 
contact policies, adapting curriculum, development of an accountability plan, and introduction of mixed-gender co-facilitation. For instance, one participant explained that while their program's philosophical alignment with standards was present from the beginning, in order to comply they had to translate this philosophical alignment into practice, "In content we were pretty much all in agreement [with the standards] on the things that needed to be covered... it was more around making sure we had the policies and procedures in place". Another participant indicated that some changes had to made in order to ensure the program was in compliance with standards. Specifically, "Obviously there were curriculum adjustments, policy adjustments, procedure adjustments". A third participant described their decision to utilize a new facilitation configuration as a step towards compliance, "I guess the big [change we made] was the other facilitator and I started doing this together". This information points to the fact that programs were not in complete compliance prior to the standards or when the program began providing services. Because of this, changes had to be made in order to achieve greater adherence to the standards. In addition to internal program changes in order to meet the requirements of standards, participants also described external activities that took place with the goal of increasing compliance.

The next most common implementation strategy discussed by participants was attending trainings. Six participants (45\%) reported that they attended trainings as a direct result of the standards. One participant noted that training became a priority despite lack of funding, "Even though I have limited resources I pay to have [staff] go out and get trained". This participant also noted that due to the standards they seek out training even 
in instances where they would prefer a different training source, "I send people [to trainings] within the state. Even though I'm not philosophically tightly aligned with the programs providing training...I still send people to the trainings". Another participant noted that they began attending trainings in order to comply with the standards, "[To implement the standards] I started going to the county trainings that the victim advocates were putting on”. These experiences point to a concrete action step taken by participants that requires both time and financial resources. Compliance with standards appears to be important enough for nearly half of the providers to have sought out trainings. Importantly, a third participant noted that while they have tried to seek out trainings in order to align with the standards, it has been a difficult process. Specifically they reported, "I called [a victim advocacy program] here and [another victim advocacy program], and then [a different victim advocacy program]. I had a whole list here for months. I don't know where to get victims training". Thus, even when trainings are sought out, it may not always result in compliance with that component of standards.

In addition to attempts to obtain training, participants also described putting on trainings in order to facilitate meeting the requirements of the standards. Three participants $(23 \%)$ indicated they hosted trainings in order to achieve training requirements or build relationships. One participant described:

I started with meeting with advocates first. Then I first submitted a letter to domestic violence council folks about what [training] I could do... And then I did a training for the community, or key stakeholders, on the dynamics and on the issues [of batterer intervention].

Another participant explained that providing trainings for those involved in the collaborative response has been helpful to maintain collaborations required in the 
standards, "We found it has worked really well to invite victims advocates... and train them in batterers intervention. We've had some drug and alcohol counselors who were interested in [batterers intervention] and we train them on that side of things". A third participant discussed hosting a training in order to meet the training requirement, "We worked with probation and victim services and we put on a forty hour training". It appears that hosting trainings is a beneficial activity to facilitate compliance with both the training and collaboration requirements of the standards.

While hosting trainings was one way of facilitating collaboration, participants also discussed fostering relationships more generally to implement the standards. Five participants (38\%) described attempts to create or improve relationships with collaborative partners in order to facilitate compliance. One participant explained, "I think there's been an attempt to do the collaborative work and let other people know what I do and how I do what I do", they go on to say, "We had advocates coming and sitting in group, we would go talk to women's groups. There's always been dialogue with the referral sources but I see that as more organized now". Another participant described the development of collaboration with staff from other BIPs:

We began to work as a group and we all got on the same page together. And then it was much more smooth. We'll sit in there as a group and we'll ask the other batterer intervention [providers], what do you charge for this? And how do you word this in your contract? So we share that information rather than having this competition between us.

This comment points to the value of collaboration within the BIP community. For this participant the standards helped facilitate building relationships with other BIPs and this has been a valuable resource for this participant. It is important to note that competition 
and lack of collaboration among BIP providers was raised during the interviews and appears to be viewed as a concern among some providers. Thus, it may be beneficial for the field if the implementation of standards can foster these relationships and the education that occurs in them. A third participant noted that because of the standards, "I think we are more tied in with our community groups". All of these comments highlight the emphasis placed on collaboration by participants. Collaboration appears to be one component of the standards that participants actively attempted to improve in order to ensure compliance and many participants described these attempts as at least somewhat successful in building relationships.

Perhaps it is not surprising that the most straightforward response to the question of how participants have implemented the standards - reading them, carefully and often - was voiced by five participants (38\%). For instance one participant explained, "I read them thoroughly. I underline things. I make sure that I understand what they're asking. I take them very seriously". Another participant echoed this:

I want to stay on top of them. I periodically read them... as they come out and see what, if any, changes. That's made it easy for me in implementing them just to continually read them and go over them and make sure that I'm doing the best... that I can, make sure that I've got things in place.

While simple, just reviewing the standards and using them as a guide to ensure practices meet expectations is a strategy used by some participants.

Finally, the hiring of additional staff was another implementation strategy discussed by two participants (15\%). These participants indicated that in order to meet the requirements set forth in the standards they hired an additional facilitator to provide services. One participant reported hiring an intern to serve as a co-facilitator, 'I'm just 
hoping maybe this [person]... will help me with the problem I've got of not having an [opposite sex] perspective". A second provider reported hiring a new facilitator, "We brought in a(n) [opposite sex] facilitator so that we have co-facilitation”. It appears that for some programs, the hiring of additional individuals is one way to increase compliance with the standards. An additional staff member can provide greater capacity to utilize mixed gender co-facilitation in groups and provides an additional person to participate in components of the community collaborative response. For instance, additional staff may increase capabilities to attend meetings held by the DV council and communicate with the DV council, other BIP providers, corrections, or victim advocates. While this may be a somewhat expensive implementation strategy, for a minority of programs it did allow them to reach greater compliance with the standards.

\section{Section 8: Implementation Ease and Difficulty}

Implementation ease $(\kappa=.66)$ was coded when participants named practices that already aligned with the standards and thus did not require change, or practices that were relatively easy to change. The code of implementation ease was coded 67 times, with frequency ranging from $0-13$ instances per participant $(M=5.15, S D=3.58)$. Almost every participant $(n=12 ; 92 \%)$ described at least one instance of implementation ease. Of these 12 participants, implementation ease was coded an average of $5.58(S D=3.37)$ times. Implementation difficulty $(\kappa=.65)$ was coded when participants named practices that were relatively difficult to change or remain noncompliant because they are perceived to be too difficult to change. The code of implementation difficulty was coded 69 times, with frequency ranging from $1-12$ instances per participant $(M=5.31, S D=$ 
3.15). All 13 participants described at least one instance of implementation difficulty. It is important to note that implementation difficulty was the most commonly used theme. Thus, it is clear that participants had a great deal of insight and information related to which components of the standards have been most difficult to comply. These findings indicate that participants viewed some components of the standards as relatively problematic to implement, while other components were relatively simple to implement. In order to fully understand the distinction between implementation ease and implementation difficulty, the specific content of coded material was examined.

Implementation ease. The content coded for implementation ease was examined in detail to determine the types of requirements that participants perceived as relatively simple to implement. It is important to note that a relatively large proportion $(n=9,69 \%)$ of participants made at least one overarching statement indicating that most components of the standards were already in place or easy to implement. These comments included statements such as, "Most... everything that the standards require we had in place before the standards came out, so it wasn't really a challenge" and "As far as changing, we really haven't changed. We've really remained pretty consistent'. Thus, it appears that the majority of participants believed that the standards overall were not problematic to implement.

In addition to these general statements indicating implementation was not too demanding, participants also described specific components of the standards that were already in place or easy to achieve. Most commonly $(n=9,69 \%)$, participants indicated that shifting program length to be in alignment with the standards was relatively easy. As 
one participant explained, "Obviously the length was the easiest to understand and to implement for us because we were already doing it before it actually became a law. So, we were happy with it, we were already doing it". Another participant reiterates this, "We had already decided that we were going to be 48 weeks". For these participants, a program length of 48 weeks was already established, decided on, or easy to understand and therefore implement. Related to program length, implementation of the requirement for monthly aftercare sessions, was also named by two participants (17\%) as unproblematic. For instance, "We were tasked with developing an aftercare program [which was relatively easy]". Together, these experiences indicate that the concrete requirements related to length of intervention were noted as components of the standards that were easier to put in place.

Participants described other components of the standards they believed were easy to implement beyond program length and aftercare. Another commonly discussed component was alignment in program philosophy and curriculum. Seven participants $(54 \%)$ indicated that program philosophy or curriculum was relatively easy to bring into line with the standards. When asked what was easy to implement, one participant described their alignment with the guidelines surrounding curriculum, "Content... I mean that's the kind of core stuff that is pretty easy. I don't know how you run a batterer's program and not be in line with those standards of the curriculum”. A second participant explained that it was easy to differentiate different approaches to intervention, "Making the distinction between anger management versus batterer intervention [was easy]". Additionally, three participants $(23 \%)$ reported that the introduction of a mandated 
accountability plan was straightforward. When asked to describe what was easy to implement one participant explained, "The accountability plan and helping the guys be in tune with each other and hold each other accountable. Those things were already in place". For most participants it appears that the contextual beliefs regarding the dynamics of IPV, as well as appropriate approaches to intervention, was easy to implement. In addition to ease implementing guidelines related to philosophy and approach, less than half of the participants $(n=5,38 \%)$ indicated collaboration with community partners was not problematic. When asked to describe what components of the standards were easy to implement, one participant noted, "It's really easy to develop working relationships with your shelter and your advocacy programs”. A second participant described improvement in collaboration after the standards were introduced, "I would say that in general our interactions with victims' advocates are less conflictual (sic) now than they were". An additional participant indicated collaboration was easy because relationships were already established, "Easiest for me is the interaction with the local domestic violence team... it was easier for me to adopt because it was already in place when I came here". For the minority of participants, collaboration was not problematic. This appears to be the result of longstanding relationships, as well as improvement in relationships due to the introduction of standards.

A subset of participants $(n=3,23 \%)$ reported that achieving the training required by the standards has not been difficult. One participant described this as being easy because foundations were already there for their staff, "I got my 40 hours of victim [training previously]... so I already had that. [My staff] came from victim service 
[backgrounds] so they had that training". Another provider reiterated ease because of prior program practices, "We were already doing the 40 hours of training for all staff". While less than one-quarter of participants reported training requirements were easy to implement, these participants reported this was the case because features of the staff or program that were already in place were conducive to meeting the training requirements. The final component that participants indicated was easy to implement was that of cofacilitation. Similar to training, only a small number of participants $(n=3,23 \%)$ reported this requirement was easy to achieve. When asked which components were easy to implement, these participants reported, "We were already doing co-facilitation", "The mixed-gender thing", and "Having a co-therapist".

Implementation difficulty. In addition to understanding which components participants perceived as easy to implement, the content of responses regarding what was difficult to implement was also assessed. All participants discussed at least one component of the standards that was difficult to implement. While the majority of participants made global statements indicating the standards overall were easy to implement, only two participants $(15 \%)$ indicated that most components were difficult to implement. These participants reported, "I think most of the standards are not easily implemented" and "Now I just operate outside of the law". For these two participants, the overall set of standards has been problematic for implementation. While one participant indicated their program tried to achieve standards with limited success, the second participant indicated that the standards are so difficult to achieve they have stopped trying and instead run their program how they see fit. 
While few participants noted the standards overall were difficult to implement, various components were discussed as problematic for implementation. Consistent with the least frequently described easy to implement components (e.g., training and cofacilitation), the most commonly described difficult to implement components were achieving training requirements and co-facilitation. Seven participants (54\%) indicated achieving training requirements was difficult or an area that remains out of compliance. One provider described where their program fell short of compliance, "[There is] probably some looseness around the training and the number of hours dedicated to victim advocacy and further batterer intervention training". Another participant explained their difficulty:

Getting training hours [is most difficult]. It's because some of the training is unavailable. That's been the number one thing. And not only the specific training for facilitators but also not getting enough hours in group to match what they need. Let's say they are only training in one group and that's an hour and a half a week, they need 150 hours, that takes a long time.

It appears that training may be a difficult component to achieve in certain locations where trainings do not often occur, the number of groups is very small, or funding sources are especially limited.

The second difficult component that was mentioned most frequently was the implementation of mixed-gender co-facilitation. Seven participants (54\%) reported this facilitation strategy was difficult to implement. One participant recognized that facilitation strategy is an area of noncompliance for their program, "The [opposite sex] co-therapist, I know, is a deficiency in terms of where I want to be and where I am”. Another participant indicated that program characteristics make this component difficult 
to achieve, "Ideally we would like a co-facilitator. Unfortunately, we don't have the resources in a rural area like this to do that". Similarly, a different participant mentioned, "[It is difficult] trying to have male-female co-facilitation in a rural community". These experiences indicate that rural programs may have an especially difficult time implementing co-facilitation with a male and female co-facilitator.

Next, participants indicated that components of the curriculum or philosophy supported by the standards were difficult to implement. Specifically, four participants (31\%) reported implementation difficulty related to curriculum or philosophy. One participant noted, "I think politically it's been a difficulty in terms of the development of our curricula. It's been challenging”. Another provider described difficulty in implementation because they were dedicated to maintaining family counseling in rare instances, though they are aware it is in violation of the standards, "We do family counseling... we offer it from the very beginning and we bring it up in groups. I've done that with two people, where they wanted some family counseling". This subset of participants has struggled to bring their curriculum and approach in line with what is required in the standards.

About one-quarter of participants $(n=3 ; 23 \%)$ reported difficulties implementing requirements around program length, collaboration with community partners, and/or the number of individuals in each group. Two participants who reported difficulty adhering to the length requirement indicated that this was due to discrepancies between state standards and local county standards for mandated length. For instance, one participant reported that when the standards were introduced they began to change their program 
length requirements but this transition was interrupted when local standards were created, "We were obviously [changing] our 24 week program into a 48 week program... but we really didn't get very far [with that due to county standards]". The third participant who indicated they were not in complete compliance with program length indicated that for clients referred by the Department of Health Services (DHS), the shortened county requirements are emulated, "The set of criteria that they came out with in [one county], I just adopted it and kind of morphed it into what was effective for me and my program". Thus, the primary impediment to achieving the program length requirement appears to be county standards or providers utilizing county standards as they see fit for clients who are not court referred.

Three participants also raised difficulties implementing the community collaboration components of the standards. One participant described their own implementation of collaboration as inadequate, "The community collaborative [response], I don't think I'm doing that as well as I could". A second provider reported that collaboration is a difficult task, "Collaboration and working with the community is the hardest". Further, one provider indicated finding a good match for their agency is problematic, "[It's hard] trying to find a victim advocacy agency that works well with these men and this agency". The involvement of other agencies in order to achieve a collaborative relationship likely makes it a more difficult component of the standards as compared to components that can be achieved within the program.

Implementation difficulties related to the number of individuals permitted per group were also highlighted by three participants. The standards indicate that groups 
should have between seven and twelve participants per group, with an absolute maximum of 15 participants (ODOJ, 2009). One participant explained that this is problematic:

The standards say 15 people per class... when you have only two or three people on staff, your class is as big as your class is. They do fluctuate but for the most part we probably average 20 people per class, which puts us out of the standards.

Another participant explained that the mandate for aftercare poses difficulties

maintaining group size:

With the state standards [mandating aftercare] once a month for three months... it made it really difficult for us to monitor group size... Every now and then you have this one person coming in once a month, so do you not fill the twelfth seat or maybe the eleventh seat because somebody might be coming once a month? We can't necessarily do that because from a small business perspective, you kinda gotta fill seats.

The final participant reiterated the dilemma that arises when you have more clients than spaces, "I know I have 23 guys in the group. That should not be allowed, but then what do you do?". These experiences highlight the difficulties that arise when small programs serve communities in need of services. The difficulty raises the question as to whether strict maintenance of small groups is preferred over ensuring all mandated individuals are enrolled in intervention.

\section{Section 9: Enablers to Compliance}

Enablers to compliance $(\kappa=.74)$ was coded when participants described agencies or relationships, activities, content of the standards, or program and personal characteristics which they believed aided implementation and compliance. The code of enablers to compliance was coded 53 times, with frequency ranging from $0-11$ instances per participant $(M=4.08, S D=3.52)$. The vast majority of participants $(n=11$; $85 \%$ ) described at least one instance of enablers to compliance. Of these 11 participants, 
enablers to compliance was coded an average of $4.82(S D=3.31)$ times. Participant responses included a variety of types of enablers that can be described by four categories: agency enablers, activity enablers, content enablers, and program enablers.

Agency enablers include descriptions of specific agencies or relationships with agencies that have made implementation of the standards easier. Eight participants $(62 \%)$ noted at least one agency enabler. These included indications that entities such as corrections, probation, DV court, BIP provider associations, DV councils, and victim services have enabled more successful implementation. For instance, when asked what has facilitated implementation one participant described, "Corrections... I mean just the fact that they'll even give me the proposed standards and talk to me about it is great. I'd call that support". While probation and corrections were brought up by several providers, other agencies were also discussed. For instance, one provider reported that, "Victim services wants us to do the state standards" and this helped facilitate compliance. Participants also reported that collaboration more generally has been beneficial. For instance one participant noted community partners' openness to revising aspects of their relationship based on the standards has been helpful:

The key stakeholders in our community are supportive, you know, are willing to look at whatever suggestions we're making. If we were to say something like, well, the standards say it should be this way, then they would say okay.

Another participant reinforced the importance of collaborative partners, "The only thing that has made a difference or made it any easier is jut the cooperation we have from other key stakeholders in the community". All of these experiences indicate that for the 
majority of participants, having strong relationships with key agencies has been useful for improving compliance.

In addition to agencies that aid in implementation, participants also discussed activities that have encouraged increased compliance. Five participants (38\%) reported at least one activity that was beneficial for implementation. These activities included responding to monitoring from probation, participation on the Standards Advisory Committee, attending trainings, and attending meetings with stakeholders. Monitoring from probation appears to have taken different forms across counties and three participants indicated that monitoring did facilitate greater compliance. According to one participant, their county requested programs complete a questionnaire about program characteristics and practices:

The local supervising agency put out this questionnaire a couple years ago that was really effective in being able to get me focused on what I was doing and what it was that I wasn't doing by asking specific questions about the standards and compliance with the standards.

Another participant indicated that oversight by corrections has enabled greater implementation, "I think in some ways the involvement of PO monitoring... has been challenging but helpful". A third participant reported that their county reviewed their program documents, "They wanted a copy of our contract and then had... things they wanted us to comply with. I immediately just did [the changes] and sent them this thing [saying] we made these changes". Thus it appears that even when monitoring requires additional work, it can be effective in changing practices to increase compliance.

Other activities that increased implementation and compliance mentioned by participants include participation in opportunities to interact around the standards. 
Specifically, two participants noted that participation on the Standards Advisory

Committee facilitated their programs' compliance with standards. For example, "I kept the agency aware of changes before they happened. I've also tried to get feedback from them about the direction they would like to see things go". It is perhaps unsurprising that helping shape the standards appears to help facilitate implementation, but these experiences did increase implementation and compliance for two participants' programs. Two participants reported that attendance at trainings or BIP provider meetings facilitates their ability to implement the standards because they are able to learn more about the standards and gain a better understanding of how others are implementing the various components. One participant explained, "I think that meetings like the batterers intervention trainings, the [batterer intervention provider association meetings]... help to keep us all focused on making sure what we're meeting [the standards]'. Together this indicates that for slightly less than a third of participants, activities centered on interaction with key agencies (e.g., monitoring, meetings, or trainings) were valuable in encouraging compliance.

Only two participants reported that the content of the standards or the way in which the standards were written facilitated compliance. One participant appreciated the standards as a roadmap for developing a program:

[The standards] made it very easy to put the program together because there was the teeth of what I needed contained in the document. I think the expectations were fairly clear of what each program should contain and how they should go about providing services.

Having a clear set of regulations made developing a standards compliant program less difficult for this participant. An additional participant reported that the lack of clarity in 
the standards regarding the consequences of noncompliance created anxiety, which in turn increased implementation efforts. Specifically, in response to the questions asking about enablers to compliance this respondent reported, "Fear. If there is a standard and I'm not meeting it I'm gonna be in trouble somewhere along the line. I'd better do something about it. It's terrible, but yeah, fear'. As the standards are written now, it appears that while they may serve as a guide to program development, their ambiguity regarding consequences to compliance could elicit greater implementation due to anxiety over what consequences for non-compliance will be.

Finally, eight participants $(62 \%)$ described a characteristic of their program or staff that enabled compliance with the standards. These enablers included personal knowledge of the standards, support from the agency in which the program resides, personal expertise or training, and personal characteristics. For instance, one participant noted that the larger agency's interest in batterer intervention has been helpful, "The agency's support [is an enabler]. The agency has been committed to this since the 90 s". Another participant indicated that resources at their program are sufficient to meet the requirements of the standards, "It's funded well enough so you can do stuff... Funding and staff and all the things that come with that so that you can develop some competency and deliberateness [around the standards]". A third participant echoed support within their program for components of the standards:

The agency hasn't complained a lot. At first they were like, two facilitators in one group? You know, just thinking of money. But I think they see the benefits of it and they're willing to try and help us meet the standards. 
It seems that having organizational support to meet the standards is helpful for facilitating compliance. Participants also reported individual qualities that promoted compliance, "[A enabler is] probably just me being [obsessive] about making sure I'm following guidelines like I'm supposed to". Another participant noted that their specific background made it easier to meet the standards, "I look at my area of expertise. I look at what I know about domestic violence. I look at my philosophy... [these align with] the standards". For most participants, qualities about their program or about themselves in some way facilitated their compliance with standards.

During interview data collection, it became apparent that for some individuals, participating in the research process has helped facilitate implementation and compliance. Thus, in addition to enablers reported by participants, a post-hoc code was added to the codebook to capture experiences in which the social action research philosophy of the current study was realized. Specifically, the code of social action research $(\kappa=.85)$ captured experiences where completion of the BIP survey or the interview process impacted participants or changed program practices. This code was applied 26 times, with frequency ranging from $0-9$ instances per participant. On average, participants made $2.00(S D=2.80)$ comments indicative of social action research. Six participants (46\%) made at least one comment relevant to the social action research code and among these participants the code was applied an average of $4.33(S D=2.58)$ times.

Material coded as social action research included participants' discussion of different aspects of the project that they felt impacted their ability to implement and comply with standards. This included the creation of the Oregon BIP Directory, 
education about the standards through completion of the BIP survey, increased selfawareness during the interview process, and validation of participant effort through engagement with the BIP community. Two participants explicitly referenced the Oregon BIP Directory and their appreciation for that resource. For example one participant remarked, "I appreciate that you are here and I appreciate everything that you do in creating the directory. I use that a lot when I refer clients to other programs". Five participants mentioned that completing the survey was valuable and provided education about the various topics covered in the standards. One participant in particular was moved to action after completing the survey:

Some good that comes out of what you're doing is that when I [completed] the survey, I had to fill out stuff and I had to talk to somebody on the phone about the survey and it made my butterflies turn a bit. I thought, hmm, you know I had to say no to a bunch of these [questions] on here.... One of the questions was do you have [policies and procedures] in writing and I thought, no... I thought I better get it in writing. So I got in in writing because of that survey and phone call that asked me that question. So here are my policies and procedures.

This participant then shared a 28-page document that they had created outlining all policies and procedures for their program. For this participant, the process of completing the survey was not only informative but this experience was also a catalyst for change. Other participants referred to the survey as educational. For instance, "I learned some of the standards just by taking your survey, it was a good survey". The comments indicate that the product of the Oregon BIP Directory, which was generated through conversations prior to survey completion, and completion of the survey were each beneficial experiences that impacted knowledge and practice. 
In addition to the survey, two participants also indicated that the interview itself had highlighted discrepancies in what they thought they knew. One participant explained that prior to the interview he or she had thought they were very aware of what the standards entailed but the interview made them realize they may have more to learn:

This experience alone is heightening anxiety. You know, it's like, wow. You thought you knew this stuff and apparently you don't because listen to how vague your answers are as you are trying... struggling... to address [the questions]... you know though, I am really excited and really glad that this is happening.

Thus, the interactive experience of having to answer questions directly to another person appears to have helped some participants become more aware of what they still need to learn. Finally, two participants mentioned the value of just seeing someone who is interested in the work they are doing. One provider explained how it made them feel, "[The survey and interview] made me feel like somebody [cares]... Somebody's asking my opinion and wants to know how I am doing something. It made me feel like I had a voice, which I haven't really felt like otherwise". These comments emphasize the importance of education and engagement among BIP providers. Simple steps, such as researchers calling providers to confirm addresses, surveying practices, or talking with them to learn more about how they think the BIP community should function may increase their awareness of the standards and impact how seriously they think about the different components of the standards. The social action research component of the current project appears to be a valuable enabler to compliance and implementation, especially in combination with other enablers mentioned by participants. 
Section 10: Barriers to Compliance

Barriers to compliance $(\kappa=.62)$ was coded when participants described agencies or relationships, activities, content of the standards, or program and personal characteristics which they believed hindered implementation and compliance. The code barriers to compliance was coded 105 times, with frequency ranging from $2-21$ instances per participant. All 13 participants (100\%) described at least one experience consistent with barriers to compliance $(M=8.08, S D=5.12)$. Participant responses included a variety of barriers that comprised four categories: agency barriers, activity barriers, content barriers, and program barriers.

Agency barriers included barriers to compliance that described difficulties meeting components of the standards due to problematic or lack of relevant relationships with community partners. Nine participants (69\%) had the barriers to compliance code applied at least once when discussing difficulty establishing or maintaining necessary relationships. These experiences included lack of responsiveness from community partners, negative relationships with community partners, focus on business in the BIP community as opposed to a focus on change, and conflict with local supervisory authorities (LSAs) in counties with local standards. Several participants noted that they have made attempts to work with community partners required for a community collaborative response but do not always receive a response from those to whom they reach out. One participant explained that despite contacting a victim advocacy agency, the community partner, “... just never made time to review my stuff. I mean, I tried for two years. I even had a memorandum of understanding written up so that we could [work 
together] and [they] just never read my stuff". Another participant described a similar experience:

I got on the phone to domestic violence services and I said, is there a [dv council] here that I'm not aware of? And they said, we don't have anything. I said, well, maybe we need to get something going here, I'm going to be out of compliance if I don't, it looks to me like [the standards say] I have to have some kind of involvement. [They] said, great idea... [we've] been wanting to do something like this and I am going to call my supervisor to see if we can get something [started]. [They] never called me back.

Thus, one aspect of this barrier is the difficulty in establishing relationships among different community partners that may be busy and have limited time to develop new relationships.

Not only did providers discuss lack of response, but they also described feeling unwelcome or devalued by some community partners. One participant explained that tension remains despite attempts to develop relationships with victim advocacy agencies, "I've done things to bring into their awareness what my program does... but again, because of some external issues I believe there was not a warm reception or even a reception at all in some cases". Another participant voiced their feelings that collaboration with their local victim advocacy organization isn't optimal, "I'm not being collaborated with. I'm the one doing the collaboration. I'm the one that supports them. They don't particularly support". Two participants noted that difficulties with collaboration are not exclusive to other types of agencies but are also present within the BIP community. One participant reported that the culture of the BIP community as they see it opposes the collaborative goals of the standards:

When you get away from the community perspective and you get to be in business there's that competition that comes up. The jealously and envy and 
resentment and entitlement. I think it's difficult to do this work and have [a] collaborative spirit.

A second participant summarized their view of the BIP community:

The whole idea of it being a community and it being supportive is only there at a very surface level... It has become very much a system and it's become institutionalized and when things become institutionalized we are out to protect the institution and not necessarily to help the people. It has to be much, much more collaborative than it is now. And it is not collaborative. It's very much proprietary and very much split.

These comments point to feelings of competitiveness within the BIP provider community that may be counterproductive when collaboration is a primary goal. Some providers experienced the BIP community as one that was not aimed at working together to address IPV, but instead focused on working in isolation to have a successful business. Thus, it appears that participants experience barriers to compliance due to problematic collaboration with different types of agencies, including other BIPs.

In addition to relationships with agencies that participants found problematic for compliance, participants also mentioned activities that made implementation difficult to achieve. Seven participants (54\%) reported at least one activity required by the standards that they viewed as a barrier to successful implementation. The activity coded as barriers to compliance was almost exclusively related to training for facilitators but a small number of participants described male female co-facilitation or attending collaborative meetings as an activity that makes compliance difficult. When discussing trainings, several issues were raised including lack of trainings, difficulty accessing trainings, and cost associated with trainings. For instance, one participant described the lack of available trainings as problematic, "[A barrier is] making sure that all of our facilitators 
are getting the training they need, even though there is some training that is really not available out there, so we can't do it all". A second participant reiterated this point and sought advice, "[Victim advocacy training] is a hard training to find. If you've got any ideas, I'll be glad to go do it". This participant appeared to be willing to attend trainings but needed help identifying appropriate training opportunities. When trainings are available other factors such as time and cost become salient: "[The training] was Monday through Friday all day and they were charging $\$ 100$ a day and most of us couldn't take the time off to do that. And, it's very, very expensive". Similarly a different participant noted, "Getting all those trainings [is a barrier]. I don't know where I'm going to get [all the training]. For two of us it's 160 hours of training. Coming up with the funding and the time [is hard]". Another participant summarized the difficulties finding and attaining training, "Just finding resources to get continuing education that are fairly close and not too expensive [is a barrier]". Participants consistently described achieving the training component of the standards as difficult and a barrier to their program successfully complying with standards. When male female co-facilitation was brought forth as a barrier, participants saw the cost associated with this practice as limiting, "In terms of making a living, [the standards are] not a real viable thing. A lot of that comes from the requirement of co-facilitators". For some programs, requiring that two individuals run groups prohibits the extent to which they can reach compliance with the standards. The final activity raised by participants was that of attending collaborative meetings. Again, this activity barrier was tied to time and resources, "That community collaborative component [is a barrier] trying to get the time to get everybody together". These 
experiences highlight difficulties reaching compliance due to lack of program information, access, or resources which make achieving time and resource intensive components of the standards, such as training and co-facilitation, difficult.

While relationships with relevant agencies and completing the appropriate activities can be problematic, the ways in which the standards were written can also pose difficulties. Seven participants (54\%) reported at least one way that the standards are written that makes it more challenging to achieve compliance. Four of these participants mentioned misalignment between local and state standards as something that is problematic and stems from the way standards were written, monitored, and enforced. Specifically, participants indicated that county requirements imposed difficulties determining whether it is appropriate to follow the standards by complying with requests made by the LSA when the LSA expects different requirements than those included in the standards. Specifically, one barrier listed was, “The LSA component and who's the overseeing entity". Further, when local bodies monitor and enforce local requirements, those often become more immediately important than what is required by the state. Participants who discussed this as a barrier indicated that they knew they were out of compliance in some areas but in order to comply with local regulations they had to disregard some components of the state standards, especially required program length. One participant described this barrier very simply, "Following the county [requirements] violates state standards". When asked to describe how this happened, the participant explained, "In the state standards there is no mechanism [to enforce the standards]. And there is no mechanism of what to do if a county is not following these standards". 
Another participant raised barriers due to confusion among courts in other counties because of discrepancies in requirements across different areas, "When you look at different counties who... have a 12 week [program length] option [you realize] it's not the law. Then you get the judges asking about it". Thus, discrepancies between local and state standards not only impact the specific areas where local standards are in place, but also create confusion in other areas. These experiences indicate that the lack of synergy between state standards and local requirements can cause confusion about what truly is law and with which entity programs are expected to comply. In addition to confusion regarding local standards, participants also indicated that the way that the standards are written is confusing and difficult to understand, which in turn creates a barrier. One participant described, "[The standards are] just so convoluted and it's so confusing" and "It's difficult trying to decipher the whole packet". Another participant reinforced that the structure of the standards is a barrier, "The standards are so complicated. They are so cumbersome. They are, from my point of view...if they are not already, they are quickly becoming unworkable". These participants believed that lack of clarity and simplicity in the writing of the standards made it more difficult for them to achieve compliance.

The last category of barriers to compliance described by participants included barriers due to program or personal characteristics. These barriers included difficulties due to rural location, program size, and lack of funding. Nine participants (69\%) mentioned at least one barrier that was related to program characteristics. One barrier that was described as a difficult activity to achieve, trainings, was also explained in the 
context of program characteristics. For instance, one participant explained what they

viewed as challenging for some programs:

I think some of [the programs] in rural counties have a harder time following the guidelines because I think there's even less available to them. Even less training available to them, it's more costly, they have to go out of town to get the training.

Another participant described further difficulties when they are not able to support staff with fulltime positions:

We don't have anybody who works fulltime here.... So finding somebody who's willing to put in that many hours of training and skip days of work to go to training... so that they can facilitate one night a week [is a barrier].

Participants also reported that geographic location made activities like collaboration or trainings difficult. One participant explained, "Eastern Oregon itself [is a barrier]. We're kind of forgotten about out here". Another participant described the cost associated with attending trainings due to their distance from the urban locations where most trainings are held:

[Going to trainings is a barrier] especially when you live in a [rural] area and have to travel long distances. I spend a thousand dollars when I [go to trainings] for two days with the hotel, training fees, travel, and loss of income from stopping groups while I'm gone.

For providers that do not have a large staff, attending trainings involves more than the actual cost of travel, lodging, and fees for the training because they may not have anyone to provide services while they are gone. This is likely quite different than experiences achieving trainings for providers in large programs in urban locations where they don't have to pay for travel or lodging and have other staff to help facilitate the groups they miss during training. Barriers tied to rural location go beyond trainings. For instance, "[In urban areas] they have a wide variety of interns they can get from the colleges up there. 
You don't get that in a rural community. In a rural community you can't even find space sometimes". A participant from a small program explained that a barrier stems from the lack of staff, "We're already short staffed", thus extra work that would be necessary for their program to meet the standards is difficult. These experiences point to difficulties achieving the requirements of the standards that may be even more pronounced when programs are located in rural areas, are small in size, or do not have adequate funding.

\section{Section 11: Support Needed}

Needed support $(\kappa=.73)$ was coded when participants described resources, relationships, support, or content changes they believe are necessary to maximize compliance. The code of needed support was coded 68 times, with frequency ranging from 1 - 12 instances per participant. All 13 participants (100\%) described at least one suggestion relevant to support needed $(M=5.23, S D=3.42)$.

When asked to describe what would be helpful to improve implementation and reach greater compliance, one topic that arose was that of relationship building.

Relationships were commonly mentioned as both an enabler and barrier to implementation, indicating that when relationships are supportive and cordial, they may aid in implementation and when relationships are problematic, they may disrupt implementation. Thus, it is not surprising that participants recommended creating some type of support for building and maintaining relationships in order to facilitate greater adherence to the standards. Specifically, six participants (46\%) mentioned the need for relationships in some way. Importantly, five of these six participants are from programs in rural areas where collaboration and creating connections may be most difficult. One 
participant described their desire for a more comprehensive connection to the BIP

community, "I need to connect with the network [of BIP providers]. Find out more about... meetings. Conferences. Are there BIP facilitator conferences? That's just some of the things that I don't know whether they are going on in the state right now". This participant expanded further to suggest a conference of BIP providers:

I think... the connected communication between different BIP facilitators and BIP facilities could help us all. Just having regular conferences on occasion to talk about our different programs and how we're complying with the standards and OARs and everything. I think it would be helpful across the state.

A second provider reinforced this suggestion:

I would be a neat thing if in Oregon they had some kind of organization set up where you get programs talking. At least out here, we don't talk to each other. If there was some kind of... newsletter or correspondence from people... to hear what they are doing and what they're not doing.

One participant described the utility of a membership organization that would do things

like hold conferences and facilitate communication, "A provider membership organization [responsible for] just the basics that membership organizations do. Bringing in speakers, solidifying evidence, laying it out, providing feedback... a conference. A time to get together and share". While comments surrounding enablers and barriers touched on relationships with other organizations, most participants were focused on building a network of providers in order to facilitate compliance in other areas, rather than focused on requesting support with each individual collaborative partner. These comments indicate that there is substantial interest in an organization focused on BIPs in the state of Oregon. 
Another common suggestion was to introduce some type of certification, monitoring board, or compliance specialist focused on improving compliance in supportive and non-punitive ways. Five participants (38\%) mentioned that some type of monitoring for compliance would help them with implementation. While monitoring was requested by over one-third of participants, participants specifically indicated that monitoring should be done in the spirit of improvement rather than punishment given the evolving nature of the field. One participant explained, "If I could see the [monitoring] process as being supportive and not punitive. Not just you will comply but this will be helpful to build a stronger, better program, [that would support compliance]". Another participant reflected on the fact that other states do require formal certification, "I know that there are batterers intervention providers that are certified in other states. I think that's something that probably needs to happen down the line". While participants tended to be in favor of gradual change, one participant suggested a more stringent approach to reaching compliance with standards, "Get a licensing or certification board. Make people get certified. Then if they don't, can them. You can't do this treatment if you're not going to do it right". It appears that some individuals view monitoring as a supportive improvement process while others may view monitoring as an opportunity to close programs that are not meeting minimum requirements. In line with the barrier regarding state versus local requirements, two of the individuals who supported monitoring were especially interested in state monitoring in order to encourage uniformity between state and local requirements. One participant explained, "Well as a state, if they're going to [monitor compliance] statewide, I think every county should be required to do it”. 
Another participant indicated that monitoring should be handled by someone in a position of power across the state rather than at the local level to ensure compliance across all geographic locations:

If somebody who has more power than the judges came in... and there was some accountability. Somebody that said, you can't do this, you can't redirect [programs] to comply with something [other than state standards]. Somebody who had the ability to come in and say that and hold them to it, and hold them task. I think that would help a lot.

It appears that some participants feel that if compliance is a goal statewide, steps must be taken to ensure that the state standards are actually the requirement programs are held do in all areas.

Over half of the participants $(n=7 ; 54 \%)$ also noted changes or additions to the content of the standards that may support them in reaching greater compliance. Participants indicated that condensed key points related to the standards or outlines to facilitate greater comprehension of the different components would be valuable. For instance, "I think having clear and succinct crib sheets about what the standards are all about and why [would be helpful]". Another participant reinforced this request, "Taking all the legal mumbo-jumbo out of the standards... Having it cut and dry. Here's what the batterer needs to do. Little bullet statements would be nice... If I could understand them better, I could implement them better". Other participants indicated that they view the field of batterer intervention as still developing and they believe the content of the standards should reflect that:

I think [it would be helpful] if they were more relevant and dynamic. Then it would seem like something important instead of static. When we' re looking to improve our program we don't look at the standards, that's not where we go. 
A second participant agreed that there needs to be space for growth:

I just think that the standards need to have wiggle room so that it's not just black and white. Obviously we're still so new in this... there's not enough research out there. You've got to leave room for people to try some innovative [techniques].

This comment coincides with comments raised related to initial responses to the standards which indicating it was too soon for standards as growth and innovation are still needed. Along with the need for flexibility, two participants indicated that in order to comply with standards they would like to see standards for female perpetrators.

Essentially these programs work with female perpetrators and were unsure how the standards applied in these situations, "We could use some guidelines [for our] women's batterers group". Additionally, one participant mentioned that better "communication between legislature and programs" would be helpful. While not explicitly a content modification, this highlights one participant's perception of being disconnected from those developing the content of the standards. Comments related to content of the standards point to the need for documentation that outlines the standards more clearly, as well as increased allowances for and appreciation of flexibility in order to ensure that standards are truly reflective of innovative best practices in the field.

Resources were also mentioned by six participants (46\%) as an area that may support programs in reaching compliance. These comments included references to financial resources, trainings, and opportunities for research. Participants had several ideas for improving access to trainings. When asked what would be helpful for compliance, one participant indicated that having some type of grant program for training or free trainings would be very useful, "Training money, or free trainings". Another 
participant suggested having training modules on the internet that individuals could complete at their own pace and in a location that is convenient, "Maybe creating some online training would be good. That way... those of us that are busy could fit it in our schedules". Another participant indicated that if the state believes the training is vital, the state should take some responsibility for hosting trainings:

If [training is] that important you would think [the state] would have some kind of yearly way to train people. [For example], in order to do what you're doing in Oregon, we're offering this resource to you and we expect you to come and get so many hours. I mean, some kind of training provided, other than going out searching for private places that are doing it.

Similarly, a participant indicated:

I would be thrilled if I just got a thing saying the committee in charge of the standards is sponsoring a workshop in [a certain location] on [a certain day] and here's the curriculum and here's the speakers and it's going to be nothing but treatment of batterers. I'd be there in a second. Most people would be.

These comments point to the need for different types of training opportunities, as well as the potential role that the Standards Advisory Committee could play in facilitating trainings that are advertised to programs across the state. Participants also mentioned that money would aid them in complying with standards but participants did not offer ideas as to where that money should come from or how it should be distributed. Thus, while money may be useful in achieving compliance, it may be an unrealistic request without further details as to how providers believe the distribution of money should work. Finally, two participants mentioned that they believe greater opportunities for research would improve evidence-based practices and in turn improve compliance, "Research [is needed]. If there was more research and more evidence out there, I think [the standards] would be supported". Perhaps supporting research on innovative techniques or program 
factors associated with reductions in recidivism would improve perceptions about the extent to which standards are based in evidence and therefore the extent to which they should be taken seriously and met.

\section{Section 12: High and Low Compliance}

Actual control. The high actual control $(\kappa=.65)$ and low actual control $(\kappa=.87)$ codes were utilized to assess actual control among high and low compliance participants. Across the seven high compliance programs, high actual control was coded 33 times, with frequency ranging from $0-12$ instances per participant $(M=4.71, S D=3.99)$. Six of the seven $(86 \%)$ participants reported at least one experience consistent with high actual control and for these participants this code was applied an average of $5.50(S D=$ 3.73) times per participant. The low actual control code was utilized 13 times with frequency ranging from $0-5$ instances per participant $(M=1.86, S D=2.04)$. Four of the seven $(57 \%)$ participants reported at least one experience consistent with low actual control and for these participants this code was applied an average of $3.25(S D=1.50)$ times per participant.

Across the six low compliance programs, high actual control was coded 13 times, with frequency ranging from $0-6$ instances per participant $(M=2.17, S D=2.14)$. Five of the six (83\%) participants reported at least one experience consistent with high actual control and for these participants this code was applied an average of $2.60(S D=2.07)$ times. The low actual control code was utilized 17 times with frequency ranging from 2 4 instances per participant. All six (100\%) participants reported at least one experience consistent with low actual control $(M=2.83, S D=.75)$. These findings demonstrate that 
descriptively, high compliance program participants reported a greater number of high actual control experiences and a lower number of low actual control experiences per participant. Next, the content of experiences of high actual control and low actual control was also assessed to determine if any patterns in the content of responses exist.

First, the content of high actual control experiences were examined in the high and low compliance program participant responses. All three participants who were or are members of the Standards Advisory Committee are from high compliance programs. An additional high compliance program participant was asked to be on the committee but declined due to time restraints. Further, another high compliance program participant reported providing direct feedback to committee members through organized meetings focused on the content of the standards. Thus, of the seven high compliance program participants, five described very direct and concrete contributions to the standards. For instance one provider remembers meetings they attended:

There was a series of meetings that they had a number of providers and corrections officers and attorneys and victim assistance or victim advocates come down to Salem and join a group and have a discussion about things that were already happening, things that were going to change, [and] things that were going to be new.

Thus, these participants tended to be very active and knowledgeable about the standards and their development.

This level of involvement was not evident among low compliance program participants. These participants tended to be much more vague when describing their knowledge of the standards process or their contributions to that process. None of these participants reported participating on the Standards Advisory Committee or providing 
direct feedback. Instead, they tended to name key members of the Standards Advisory Committee as their only indication of high actual control. For example, one participant was aware of the key policymaker behind the standards but this awareness was not the result of direct action, "I think after the fact I heard rumors like, oh it was [former Attorney General] Hardy Myers' team, but it was after the fact”. Additionally, some participants could only name other providers they thought were currently on the committee. While describing key individuals relevant to the development of the standards was coded as high actual control, the type of knowledge that high compliance and low compliance program participants have in this regard appears to be quite different.

This pattern continued when the low actual control code was examined. While four high compliance program participants reported at least one low actual control experience, their comments tended to be descriptive and note that while they were aware of the process they were not directly part of the Standards Advisory Committee. For instance one provider remembered meetings related to the standards that they did not participate in:

I didn't go to any of those [meetings] but I knew that [the standards were] in progress. All of us that are working with the counties... knew it was happening so we all needed to know when it became law.

Thus, this individual was aware that standards were being developed and knew meetings were in progress to formulate the standards, though they did not formally participate. While participation may not have always been possible for these individuals, it appears that they tended to still have awareness that the standards were being developed or discussed. When asked to describe what they knew about the standards creation process, 
low compliance program participant responses tended to be much less descriptive and included responses such as, "No clue" and "I don't know what the process was at all". Not only did these participants tend not to have an active role in the development of the standards, they also did not have a clear understanding of the process by which the standards were created.

Perceived control. In order to understand perceived control among high and low compliance programs, the high perceived ability $(\kappa=.63)$, low perceived ability $(\kappa=$ $.75)$, high procedural justice $(\kappa=.66)$, and low procedural justice $(\kappa=.55)$ codes were utilized. Across the seven high compliance programs, high perceived ability was coded 14 times, with frequency ranging from $0-4$ instances per participant $(M=2.00, S D=$ 1.41). Six of the seven (86\%) participants reported at least one experience consistent with high perceived ability and across these participants, this code was applied an average of $2.33(S D=1.21)$ times. The low perceived ability code was utilized 6 times with frequency ranging from $0-3$ instances per participant $(M=.86, S D=1.07)$. Four of the seven $(57 \%)$ participants reported at least one experience consistent with low perceived ability and when just these participants were considered, this code was applied an average of $1.50(S D=1.00)$ times. High procedural justice was coded 10 times, with frequency ranging from $0-5$ instances per participant $(M=1.43, S D=1.90)$. Four of the seven (57\%) participants reported at least one experience consistent with high procedural justice and of these participants, this code was applied an average of $2.50(S D=1.92)$ times. The low procedural justice code was utilized 22 times with frequency ranging from $0-7$ instances per participant $(M=3.14, S D=2.73)$. Five of the seven $(71 \%)$ 
participants reported at least one experience consistent with low procedural justice and of these participants, this code was applied an average of $4.40(S D=2.07)$ times.

Across the six low compliance programs, high perceived ability was coded 4 times, with frequency ranging from $0-3$ instances per participant $(M=.67, S D=1.21)$. Two of the six (33\%) participants reported at least one experience consistent with high perceived ability and for these participants, this code was applied an average of 2.00 (SD $=1.41$ ) times. The low perceived ability code was utilized 15 times with frequency ranging from $0-6$ instances per participant. All six (100\%) participants reported at least one experience consistent with low perceived ability $(M=2.50, S D=1.87)$. High procedural justice was coded 2 times, with frequency ranging from $0-2$ instances per participant. When all low compliance program participants were considered, this code was applied an average of $.33(S D=.82)$ times. Only one of the six $(17 \%)$ participants reported at least one experience consistent with high procedural justice and this participant made two comments related to high procedural justice. The low procedural justice code was utilized 10 times with frequency ranging from $0-6$ instances per participant $(M=1.67, S D=2.25)$. Four of the six $(67 \%)$ participants reported at least one experience consistent with low procedural justice. When just these participants were considered, this code was utilized an average of $2.50(S D=2.38)$ times.

Examinations of interview material coded as high perceived ability or high procedural justice revealed that high compliance program participants have more to say about their perceived control over the standards. High perceived ability was coded in a total of eight interviews, six of which were high compliance participants. High 
compliance program participants tended to be more concrete in their description of what they would do if they wanted to impact the standards. For instance, these participants already served on the Statewide Standards Committee or were aware of specific committee members with whom they could talk. When asked if their input would be heard and valued, one participant said, "I'm kind of in an odd position because I do have the ear here of some people already because of my history and herstory of me being involved in this issue for [so many] years in the state". Like this example, high compliance program participants tended to be more confident that if they were to take action, they would be able to make an impact. Conversely, while two low compliance program participants believed they could impact the standards if they wanted to, their descriptions of how they would accomplish this were much more vague. One participant described the steps they would take, "I would probably call the state and find out how to get a hold of somebody on the board for batterers or for BIPs...". Thus, while they did have some confidence in their abilities, their actual plan to create change was relatively underdeveloped.

This pattern also held in the content coded as high procedural justice. Of the five interviews where this code was applied at least once, four were from high compliance program participants. Again, while high compliance program participants tended to provide concrete rationale for believing the standards process and committee were fair, the one low compliance program participant was less concrete. For example, one high compliance program participant explained:

I think they did a general call for all providers to come and hear what they were proposing... As far as I remember and recall, the invitation was put out generally 
for providers to come and be part of this process. So, I don't think that it was exclusionary. I think there were a certain group of individuals that just made it a priority to be a part of this when it was starting up.

This is a quite different response than what was given by the low compliance program participant when asked whether the standards process was fair, "I think so". Thus, both the number of instances and the content of perceptions related to high perceived ability and high procedural justice differed across high and low compliance program participants.

Next, content coded as low perceived ability and low procedural justice was examined. While four of the seven high compliance program participant interviews had the code of low perceived ability applied at least once, all six low compliance program participant interviews were applied this code at least once. The content of these responses reveals an interesting pattern. While high compliance program participants described perceptions consistent with low perceived ability, these perceptions were typically centered on their power and ability to create change relative to others. For instance, one participant noted that they do not believe they are always taken seriously because they are vocal about their thoughts, "I think there are times when, because I have been a thorn in people's sides, that just the fact that I'm saying something, all they're hearing is blah, blah, blah". A second participant reported that they believe others have more control of the standards, "I believe that the other voices have more power". Thus, while these providers cast doubt on their ability to impact the standards, this is due to their awareness of the context and relationships with others involved in the standards process. Conversely, low compliance program participants described perceptions indicative of 
feeling they have no power or no knowledge of how to access power. When asked whether their voice would make a difference, one participant noted, "No... because I don't know who I would go to". Another participant firmly stated that they are only focused on improving compliance and would not feel comfortable trying to make changes, “I just need to meet them. I can't really fight them”. Thus, the lack of perceived ability to impact the standards tends to stem from different perspectives for high and low compliance program participants.

Low procedural justice was coded in a similar proportion of high and low compliance participant interviews ( $71 \%$ versus $67 \%$, respectively). While this is the case, the content and depth of discussion on this topic is quite different across the two groups. The high compliance program participants were much more vocal about unfairness they experienced or perceive as it relates to the standards. They had a number of critiques relating to the composition of the Standards Advisory Committee and the ways in which decisions were and are made. For example, several high compliance program participants raised issues related to the demographic composition of the committee such as, "It's...very white" and "It's a majority culture. Middle class professionals". Low compliance program participants were much more succinct and broad when the topics of fairness and representativeness were raised. For instance, when asked whether the committee represents most providers, one participant simply said, "No", and did not care to elaborate. Additionally, some of the critiques raised by low compliance program participants were not accurate. For instance, one provider believed that BIP providers were not part of the Standards Advisory Committee and did not contribute to the 
development of standards. They viewed this as a major shortcoming, though in reality several BIP providers were and are active members of the Standards Advisory Committee. Thus, while high compliance program participants voiced a higher number and greater variety of critiques related to procedural justice, this is likely due to their greater familiarity with the members of the committee and process used to create the standards.

These findings indicate that high compliance program participants reported a greater number of high perceived ability and high procedural justice experiences. Interestingly, high compliance program participants also reported a greater number of low perceived ability and low procedural justice experiences per participant. When experiences high perceived ability, low perceived ability, high procedural justice, and low procedural justice were accounted for together, high compliance program participants tend to have higher perceived control ratios.

Negative Attitude Change and Maintenance. Four codes were utilized including positive initial response $(\kappa=.64)$, negative initial response $(\kappa=.86)$, positive current response $(\kappa=.76)$, and negative current response $(\kappa=.63)$, to identify experiences those who shifted and maintained negative attitudes towards the standards. Across the seven high compliance programs, positive initial response was coded 25 times, with frequency ranging from 1 - 7 instances per participant. All seven (100\%) participants reported at least one experience consistent with positive initial response $(M=$ $3.57, S D=2.57)$. The negative initial response code was utilized 18 times with frequency ranging from $0-5$ instances per participant $(M=2.57, S D=1.62)$. Six of the seven 
(86\%) participants reported at least one experience consistent with negative initial response. When just these participants were considered, this code was applied an average of $3.00(S D=1.27)$ times. The average initial response ratio was.57 $(S D=.31$; ranging from $.17-1.00)$. This indicates that on average, $57 \%$ of these participants' comments related to initial response were indicative of positive initial response. Further, four of the seven participants reported more experiences of positive initial response compared to negative initial response evidenced by initial response ratios greater than .50 .

Positive current response was coded 67 times, with frequency ranging from 6 11 instances per participant. All seven (100\%) participants reported at least one experience consistent with positive current response $(M=9.57, S D=1.90)$. The negative current response code was utilized 77 times with frequency ranging from $5-16$ instances per participant. All seven (100\%) participants reported at least one experience consistent with negative current response $(M=11.00, S D=4.08)$. The average current response ratio for these participants was $.48(S D=.09$; ranging from $.36-.61)$. This indicates that on average, $48 \%$ of these participants' comments related to current response were indicative of positive current response. Further, three of the seven participants reported more experiences of positive current response compared to negative current response evidenced by initial response ratios greater than .50 .

Next, the six low compliance program participants were considered. Across these participants, positive initial response was coded 11 times, with frequency ranging from 0 - 3 instances per participant $(M=1.83, S D=1.17)$. Five of the six $(83 \%)$ participants reported at least one experience consistent with positive initial response and when just 
these participants were considered, the code was applied an average of $2.20(S D=.84)$ times. The negative initial response code was utilized 17 times with frequency ranging from $0-4$ instances per participant $(M=2.83, S D=1.60)$. Five of the six $(83 \%)$ participants reported at least one experience consistent with negative initial response. When these five participants were considered, this code was applied an average of 3.40 $(S D=.89)$ times. The average initial response ratio for these participants was $.46(S D=$ .32 ; ranging from $0.00-1.00$ ). This indicates that on average, $46 \%$ of these participants' comments related to initial response were indicative of positive initial response. Further, three of the six participants reported equal greater experiences of positive initial response compared to negative initial response evidenced by initial response ratios equal to or exceeding .50 .

Positive current response was coded 49 times, with frequency ranging from 3 12 instances per participant. All six (100\%) participants reported at least one experience consistent with positive current response $(M=8.17, S D=2.99)$. The negative current response code was utilized 102 times with frequency ranging from 4 - 44 instances per participant. All six (100\%) participants reported at least one experience consistent with negative current response $(M=17.00, S D=14.35)$. The average current response ratio for these participants was $.40(S D=.20$; ranging from $.06-.67)$. This indicates that on average, $40 \%$ of these participants' comments related to current response were indicative of positive current response. Further, one of the six participants reported more experiences of positive current response compared to negative current response evidenced by initial response ratios greater than .50 . 
Legitimacy. In order to assess legitimacy, six codes were examined including high procedural justice $(\kappa=.66)$, low procedural justice $(\kappa=.55)$, positive norms $(\kappa=$ $.88)$, negative norms $(\kappa=.66)$, high policy logic $(\kappa=.77)$, and low policy logic $(\kappa=.82)$. Each of these codes was first examined in the high compliance program participant responses.

As described in the context of perceived control, high procedural justice was coded 10 times. Frequency ranged from $0-5$ instances per high compliance participant $(M=1.43, S D=1.90)$. Four of the seven $(57 \%)$ participants reported at least one experience consistent with high procedural justice and for these participants exclusively, this code was applied an average of $2.50(S D=1.92)$ times. The low procedural justice code was utilized 22 times. Frequency ranged from $0-7$ instances per participant $(M=$ $3.14, S D=2.73)$. Five of the seven $(71 \%)$ participants reported at least one experience consistent with low procedural justice and among these participants, this code was applied an average of $4.40(S D=2.07)$ times per participant. Procedural justice ratios for these participants ranged from $0-.45$, with an average of .25 . This indicates that on average, $25 \%$ of these participants' comments related to procedural justice were indicative of high procedural justice. Further, all of the seven participants reported fewer experiences of high procedural justice compared to low procedural justice evidenced by initial response ratios less than .50 .

Positive norms was coded 3 times, with frequency ranging from $0-2$ instances per high compliance participant $(M=.43, S D=.79)$. Two of the seven (29\%) participants reported at least one experience consistent with positive norms and for these two 
participants, this code was applied an average of $1.50(S D=.71)$ times. The negative norms code was utilized 6 times with frequency ranging from $0-3$ instances per participant $(M=.86, S D=1.22)$. Three of the seven $(43 \%)$ participants reported at least one experience consistent with negative norms. When just these participants were considered, this code was utilized an average of $2.00(S D=1.00)$ times. The average norm ratio for these participants was $.24(S D=.21$; ranging from $0.00-.40)$. This indicates that on average, $24 \%$ of these participants' comments related to norms were indicative of positive norms. Additionally, all of the seven participants reported more experiences of negative norms compared to positive norms evidenced by norm ratios less than .50 .

High policy logic was coded 11 times, with frequency ranging from $0-3$ instances per participant $(M=1.57, S D=1.13)$. Six of the seven $(86 \%)$ participants reported at least one experience consistent with high policy logic and when these participants were considered, this code was applied an average of $1.83(S D=.98)$ times. The low policy logic code was utilized 15 times with frequency ranging from $0-9$ instances per participant $(M=2.14, S D=3.08)$. Six of the seven $(86 \%)$ participants reported at least one experience consistent with low policy logic. Across these six participants, this code was utilized an average of $2.50(S D=3.21)$ times. The average policy logic ratio for these participants was $.50(S D=.32$; ranging from $0.00-1.00)$. This indicates that on average, $50 \%$ of these participants' comments related to policy logic were indicative of high policy logic. Three of the seven participants reported more experiences of high policy logic compared to low policy logic evidenced by initial 
response ratios greater than .50 . Additionally, two participants reported an equal number of high and low policy logic perceptions (policy logic ratio $=.50$ ).

Next, the six low compliance program participants were examined. Again, as was reviewed in relation to perceived control, high procedural justice was coded 2 times, with frequency ranging from $0-2$ instances per participant $(M=.33, S D=.82)$. Only one of the six $(17 \%)$ participants reported at least one experience consistent with high procedural justice and this participant made two statements related to high procedural justice. The low procedural justice code was utilized 10 times with frequency ranging from $0-6$ instances per participant $(M=1.67, S D=2.25)$. Four of the six $(67 \%)$ participants reported at least one experience consistent with low procedural justice. Across these four participants, this code was applied an average of $2.50(S D=2.38)$ times. The average procedural justice ratio for these participants was $.17(S D=.33$; ranging from $0.00-.67)$. This indicates that on average, $17 \%$ of these participants' comments related to procedural justice were indicative of high procedural justice. Additionally, all but one of the six participants reported fewer experiences of high procedural justice compared to low procedural justice evidenced by initial response ratios less than .50 .

Positive norms was coded 5 times, with frequency ranging from $0-2$ instances per participant $(M=.83, S D=.75)$. Four of the six $(67 \%)$ participants reported at least one experience consistent with positive norms and of these participants, this code was applied an average of $1.25(S D=.50)$ times. The negative norms code was utilized six times with frequency ranging from $0-4$ instances per participant $(M=1.00, S D=1.67)$. 
Two of the six (33\%) participants reported at least one experience consistent with

negative norms. For these two participants, this code was utilized an average of 3.00 (SD $=1.41)$ times. The average norm ratio for these participants was.63 $(S D=.43$; ranging from $.20-1.00$ ). This indicates that on average, $63 \%$ of these participants' comments related to norms were indicative of positive norms. Two of the six participants reported more experiences of positive norms compared to negative norms evidenced by norm ratios greater than .50 .

High policy logic was coded 9 times, with frequency ranging from $0-4$ instances per participant $(M=1.50, S D=1.64)$. Four of the six $(67 \%)$ participants reported at least one experience consistent with high policy logic. When these four participants were considered, this code was applied an average of $2.25(S D=1.50)$ times. The low policy logic code was utilized 10 times with frequency ranging from $0-4$ instances per participant $(M=1.67, S D=1.63)$. Four of the six $(67 \%)$ participants reported at least one experience consistent with low policy logic. Across these four participants, this code was utilized an average of $2.50(S D=1.29)$ times. The average policy logic ratio for these participants was $.45(S D=.36$; ranging from $0.00-1.00)$. This indicates that on average, $45 \%$ of these participants' comments related to policy logic were indicative of high policy logic. One of the six participants reported more experiences of high policy logic compared to low policy logic evidenced by initial response ratios greater than .50 . Additionally, one participant reported an equal number of high and low policy logic perceptions (policy logic ratio $=.50$ ). 